Portland State University

PDXScholar

$1-1-2012$

\title{
Electronic Properties and Structure of Functionalized Graphene
}

Pavel Plachinda

Portland State University

Follow this and additional works at: https://pdxscholar.library.pdx.edu/open_access_etds Let us know how access to this document benefits you.

Recommended Citation

Plachinda, Pavel, "Electronic Properties and Structure of Functionalized Graphene" (2012). Dissertations and Theses. Paper 585.

https://doi.org/10.15760/etd.585

This Dissertation is brought to you for free and open access. It has been accepted for inclusion in Dissertations and Theses by an authorized administrator of PDXScholar. Please contact us if we can make this document more accessible: pdxscholar@pdx.edu. 
Electronic Properties and Structure of Functionalized Graphene

by

Pavel Plachinda

A dissertation submitted in partial fulfillment of the requirements for the degree of

Doctor of Philosophy

in

Applied Physics

Dissertation Committee:

Raj Solanki, Chair

John Freeouf

Rolf Könenkamp

Sergei Rouvimov

James Morris

Portland State University

(C) 2012 


\begin{abstract}
The trend over the last 50 years of down-scaling the silicon transistor to achieve faster computations has led to doubling of the number of transistors and computation speed over about every two years. However, this trend cannot be maintained due to the fundamental limitations of silicon as the main material for the semiconducting industry. Therefore, there is an active search for exploration of alternate materials. Among the possible candidates that can may be able to replace silicon is graphene which has recently gained the most attention. Unique properties of graphene include exceedingly high carrier mobility, tunable band gap, huge optical density of a monolayer, anomalous quantum Hall effect, and many others. To be suitable for microelectronic applications the material should be semiconductive, i.e. have a non-zero band gap. Pristine graphene is a semimetal, but by the virtue of doping the graphene surface with different molecules and radicals a band gap can be opened. Because the electronic properties of all materials are intimately related to their atomic structure, characterization of molecular and electronic structure of functionalizing groups is of high interest. The ab-inito (from the first principles) calculations provide a unique opportunity to study the influence of the dopants and thus allow exploration of the physical phenomena in functionalized graphene structures. This ability paves the road to probe the properties based on the intuitive structural information only. A great advantage of this approach lies in the opportunity for quick screening of various atomic structures. We conducted a series of ab-inito investigations of graphene functionalized with covalently and hapticly bound groups, and demonstrated possible practical
\end{abstract}


usage of functionalized graphene for microelectronic and optical applications. This investigation showed that it is possible produce band gaps in graphene (i.e., produce semiconducting graphene) of about $1 \mathrm{eV}$, without degrading the carrier mobility. This was archived by considering the influence of those adducts on electronic band structure and conductivity properties. 
This dissertation is dedicated to everyone who has contributed and been a part of enriching my education over the years and encouraged me along the way, most of all my parents. 


\section{ACKNOWLEDGEMENTS}

I would like to first acknowledge and thank my adviser, Prof. Raj Solanki, for giving me the opportunity to work on this project and continue with my education over the past two years. Thank you for your irreplaceable guidance throughout the process. Your commitment to your students and enthusiasm for teaching is not lost on any who have had the opportunity to work with you. I would also like to thank my committee members, Dr. David Evans and Prof. Sergei Rouvimov, for their collaboration in the research activities that became the foundation for this dissertation. The feedback you provided is greatly appreciated. Finally and most notably, I thank my family for their unwavering support and encouragement throughout the years. 


\title{
TABLE OF CONTENTS
}

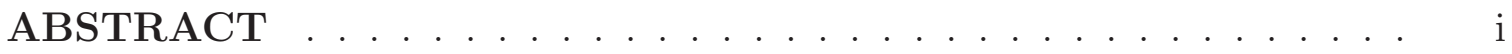

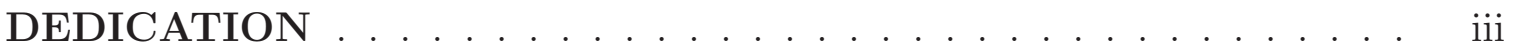

ACKNOWLEDGEMENTS ................... . . iv

LIST OF TABLES . . . . . . . . . . . . . . . . . ix

LIST OF FIGURES . . . . . . . . . . . . . . . . .

LIST OF ABBREVIATIONS ................. . . . . . . . . . . . .

CHAPTER
\end{abstract}

I. Introduction f . . . . . . . . . . . . . . . . . . 1

1.1 Demand for fast microelectronic devices . . . . . . . . . . . . 4

1.2 The Rise of Graphene . . . . . . . . . . . . . . . . . . . . 8

1.3 Chemical doping of graphene . . . . . . . . . . . . . . 12

1.4 Motivation and overview . . . . . . . . . . . . 14

II. The Density functional theory . . . . . . . . . . . . . . 15

2.1 The Hohenberg-Kohn theorem . . . . . . . . . . . . 16

2.2 The Kohn-Sham equations . . . . . . . . . . . . . . . 19

2.3 Exchange-Correlation functionals . . . . . . . . . . . . . 22

2.4 Solving the Kohn-Sham equations . . . . . . . . . . . . . 26

2.5 DFT calculation of periodic structures . . . . . . . . . . . 29

2.6 Supercells and Brillouin zone folding . . . . . . . . . . . 32

III. Quantum transport in dimensionally reduced structures . . . 36 
3.1 Electronic transport in nanostructures . . . . . . . . . . . 37

3.2 Quantum transport in the localized representation . . . . . . 44

3.3 Wannier functions and disentangled bands . . . . . . . . . 46

3.3 .1 Localization procedure . . . . . . . . . . . . . 47

3.4 Real space Hamiltonian representation and Interpolated band structure . . . . . . . . . . . . . . . . . . 48

3.5 Practical transport calculations using the NEGF $\ldots \ldots \ldots .50$

IV. Electron microscopy of graphene . . . . . . . . . . . 55

4.1 Multislice method . . . . . . . . . . . . . . . 57

4.2 Practical multislice computation . . . . . . . . . . . 62

4.3 Electron microscopy of graphene . . . . . . . . . . . 66

4.3 .1 Multilayer stacking . . . . . . . . . . . . . 67

4.3.2 Graphene Nanoribbon (GNR) . . . . . . . . . 73

4.3 .3 Adatoms . . . . . . . . . . . . . . 77

4.3 .4 Functionalizing molecules . . . . . . . . . . . 78

\section{Electronic properties of pristine graphene and graphene nanorib-}

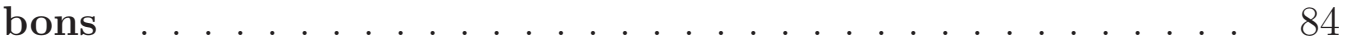

5.1 Band structure of pristine graphene. . . . . . . . . . . . 85

5.1 .1 Tight-binding model . . . . . . . . . . . . . . 85

5.1 .2 Convergence studies . . . . . . . . . . . . . . . . 90 90

5.1.3 Band structure and Density of states (DoS) . . . . 95

5.1.4 Electron density and wave functions . . . . . . . 95

5.1.5 Wannier functions in pristine graphene. . . . . . . . 99

5.1.6 Disentangled bands and ballistic quantum transport 100

5.2 Atomic and electronic structure of GNR . . . . . . . . 105

5.2.1 Atomic structure and chirality of GNRs . . . . . . 105

5.2 .2 Band structure of GNR . . . . . . . . . . . 107

5.3 Electric conductance of graphene and GNRs . . . . . . . . 110

5.3.1 Wannier function calculation . . . . . . . . . . . . 111

5.3.2 Interpolated band structure and ballistic transport . 113

5.3.3 Non-equilibrium Green's functions (NEGF) transport 114

5.4 Thermal conductivity of graphene and GNRs . . . . . . . 116

5.4.1 Ballistic and semiballistic transport in graphene . . 116

5.4.2 Phonon dispersion relations in GNR . . . . . . . . 120

5.4 .3 Scattering mechanisms in GNR . . . . . . . . . 125

5.5 Edge roughness function . . . . . . . . . . . . . . . . 128

5.5.1 Scattering on rough boundaries . . . . . . . 131

5.5.2 Thermal conductance of GNRs . . . . . . . . . . 133 
VI. Covalent functionalization of graphene . . . . . . . . . 138

6.1 Covalent functionalization of graphene. . . . . . . . . . . . 139

6.2 Epoxy functionalization . . . . . . . . . . . . . . . . . . 141

6.2.1 Band structure of epoxygraphene. . . . . . . . . . 143

6.2.2 Wannier functions and transport in epoxygraphene. 145

6.3 Amino functionalization . . . . . . . . . . . . . . . 150

6.3.1 Band structure of aminographene. . . . . . . . . . 151

6.4 PFPA functionalization . . . . . . . . . . . . . . . 153

6.4.1 Reaction and bonding between PFPA and graphene. 155

6.4.2 Band structure of PFPA-FG . . . . . . . . . 158

6.4.3 Wannier functions and transport in PFPA-FG . . . 161

6.4.4 Comparison with experimental results . . . . . . 168

6.5 Summary of covalent functionalization of graphene. . . . . . . 169

VII. Haptic functionalization of graphene . . . . . . . . . . . 171

7.1 Hapticity . . . . . . . . . . . . . . . . . . . . . . 172

7.1.1 Electronic structure of metal-bis-arene molecules . . 174

7.2 Metal-arene functionalization . . . . . . . . . . . . 177

7.3 Electronic structure MAFG . . . . . . . . . . . . . . 183

7.3.1 Electronic configuration of 1-MAFG and 4-MAFG . 183

7.3 .2 Band structure of 1-MAFG . . . . . . . . . . 183

7.3.3 Band structure of 4-MAFG . . . . . . . . . . 185

7.4 Transport in MAFG structures . . . . . . . . . . . . 187

7.4.1 Molecular orbitals of MAFG structures . . . . . . 187

7.4.2 Wannier functions of MAFG structures. . . . . . . 189

7.4.3 Transport in MAFG structures . . . . . . . . . . . 190

7.5 Magnetic properties of MAFG structures . . . . . . . . . 194

7.6 "Piano stool" functionalization . . . . . . . . . . . . 200

7.6.1 Electronic structure of MCO molecules . . . . . . 202

7.6.2 Band structure of Piano stool-FG . . . . . . . . . 205

7.6.3 Wannier functions of PS-FG structures. . . . . . . . 206

7.6.4 Transport in Piano stool-FG structures . . . . . . 208

7.7 Summary of haptic functionalization of graphene. . . . . . . . 209

VIII. Conclusion . . . . . . . . . . . . . . . . . . . . . 212

8.1 Conclusion . . . . . . . . . . . . . . . . . . . . . . . . . 213

8.1.1 Pristine graphene . . . . . . . . . . . . . . . 213

8.1.2 Covalent functionalization of graphene. . . . . . . . 214 
8.1.3 Haptic functionalization of graphene. . . . . . . . 215

8.2 Further work . . . . . . . . . . . . . . . . 216

BIBLIOGRAPHY . . . . . . . . . . . . . . . . . . 218

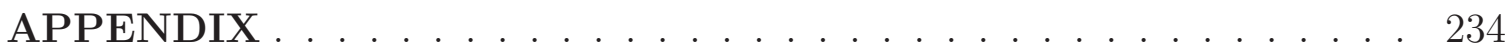

A.1 Standard parameters for the DFT calculations . . . . . . . 235

A.1.1 Geometry optimization . . . . . . . . . . 235

A.1.2 Band structure . . . . . . . . . . . . 235 


\section{LIST OF TABLES}

\section{$\underline{\text { Table }}$}

2.1 Basis sets available in $\mathrm{DMol}^{3} \ldots \ldots$. . . . . . . . . . . 28

3.1 Parameters of the Hamiltonians used for Wannier functions calculations 53

4.1 The resolution and extent in real and reciprocal space . . . . . . . . 67

5.1 Convergence studies of pristine graphene with respect to the interlayer separation and type of functional used. . . . . . . . . . . . . . 93

5.2 Geometric parameters of graphene and GNR atomic structure. . . . 108

5.3 Geometric parameters for zigzag and armchair terminations . . . . . 108

5.4 Comparison between experimental, ab-initio, and semi-empirical phonon spectra . . . . . . . . . . . . . . . . . 121

5.5 Speed of sound calculated using ab-initio, and semi-empirical phonon spectra . . . . . . . . . . . . . . . . . 125

6.1 Charge, charge transfer $(\Delta Q)$, and relative electronegativity $\left(\chi_{X} / \chi_{C}\right)$ in PFPA-FG. . . . . . . . . . . . . . . . . . . . . . 160

6.2 Summary of electronic properties produced by covalently functionalization of graphene. . . . . . . . . . . . . . . . . . . 170

7.1 Geometric parameters of free MA molecules and MA molecules bound to graphene sheet. . . . . . . . . . . . . . . . . . . . . . . . . . . 181

7.2 Electronic configuration of the metal atoms in the MA, and the corresponding energy gap opening in the MA-FG as the result of functionalization. . . . . . . . . . . . . . . . . . . . . 184

7.3 Magnetization values in FM and AFM 4-MnAFG structures . . . . 198

7.4 Electronic configuration of Piano stool-molecules as a function of electron count of the central atom. . . . . . . . . . . . . . . . . 204

7.5 Summary of electronic properties produced by covalently haptically of graphene. . . . . . . . . . . . . . . . . . . . 211 


\section{LIST OF FIGURES}

\section{Figure}

1.1 Schematic layout of the dissertation . . . . . . . . . . . 2

1.2 Plot of CPU transistor counts against dates of introduction. . . . . 6

1.3 Graphene as a 2D building material for carbon materials of all other dimensionalities. It can be wrapped up into 0D buckyballs, rolled into 1D nanotubes or stacked into 3D graphite. . . . . . . . . . .

1.4 Dispersion relations in graphene. 3D view and Contour plot of the valence band . . . . . . . . . . . . . . . . . . 11

2.1 Comparison of self-consistently calculated LDA and EXX band gaps (in $\mathrm{eV}$ ) of various semiconductors with experimental data. . . . . . 24

2.2 First Brillouin Zone of a graphene hexagonal unit cell and the IBZ part of it (blue) . . . . . . . . . . . . . . . . 31

2.3 One dimensional BZ folding in a $3 \times 1 \times 1$ super cell . . . . . . . . 33

2.4 Mapping of the second BZ of a square lattice onto the first BZ . . . 34

2.5 Mapping of the second BZ of a hexagonal lattice onto the first BZ . 35

3.1 Nanodevice coupled to semi-infinite contacts with different Fermi levels $\mu_{L}$ (left) and $\mu_{R}$ (right). $\mathbf{H}_{\mathbf{C}}$ denotes the conductor (C) Hamiltonian, $\mathbf{H}_{\mathbf{L}}$ and $\mathbf{H}_{\mathbf{R}}$ are the Hamiltonians of the left (L) and the right (R) electrodes . . . . . . . . . . . . . . . . . . 37

4.1 Schematic ray path in electron microscope . . . . . . . . . 57

4.2 The multislice approximation converts a specimen (left) into many thin slices (right) . . . . . . . . . . . . . . . . . 59

4.3 Propagation of $\delta$-like wave function through crystal slices in direct space . . . . . . . . . . . . . . . . . 61

4.4 Rearrangement of reciprocal space for Fast Fourier Transform (FFT). Stars and solids indicate relative orientations of quadrants before and after rearrangement . . . . . . . . . . . . . . 64

4.5 Crystalline models for AA- (a) and AB-stacking (b) in graphene. Perspective and top views . . . . . . . . . . . 68 
4.6 Computer simulations of HRTEM contrast of single- and bi-layer graphene (the first and second rows, respectively) at defoci of 0 and $-70 \mathrm{~nm}$ (the left and right columns, respectively) . . . . . . . . .

4.7 High Resolution Transmission Election Microscopy (HRTEM) image of graphene taken on FEI Titan on the edge of a film. The imaging conditions were set close to Schertzer focus. The image contains areas

4.8 of single and bi-layer graphene. . . . . . . . . . . . . . (c) and tetra (d)-layer graphene at defocus of $-60 \mathrm{~nm}$. Contrast on the simulated images has been maximized and, therefore, looks much higher as compared to experimental images of Fig. 4.7 . . . . . . .

4.9 HRTEM images of the edge of the graphene film: (a) raw image and (b) fragment of the same image with stick-and-ball atomic models overlaid on the edge. Computer simulations of HRTEM contrast of the "zigzag" (right) and the "armchair" (left) ribbons of single graphene layers. Imaging conditions: $U=80 \mathrm{kV}, C_{s}=0, C_{c}=0$, defocus values are shown on the left.

4.11 Computer simulations of HRTEM contrast of the armchair ribbon. (a) Only chromatic aberration included. (b) Both spherical and chromatic aberration are included. . . . . . . . . . . . . . . . .

4.12 Crystal structure of a graphene sheet and an adatom in the A (a), B (b) and C (c) position . . . . . . . . . . . . . .

4.13 (a) Contrast for an iron adatom in the A position, (b) Contrast for an iron adatom in the $\mathrm{B}$ position, (c) Contrast for an iron adatom in the $\mathrm{C}$ position. . . . . . . . . . . . . . . .

4.14 Atomic structures of functionalized graphene. (Top view). PFPA-FG (a), MCO-FG(b), MBA-FG(b) . . . . . . . . . . . . .

4.15 TEM contrast for PFPA-FG (a), MCO-FG (b), and MBA-FG (c). Defocus values are $-0 \mathrm{~nm},-15 \mathrm{~nm},-45 \mathrm{~nm},-75 \mathrm{~nm}$ (from left to right), $\mathrm{U}=80 \mathrm{kV}, \mathrm{Cc}=1.3 \mathrm{~mm}, \mathrm{Cs}=0 \ldots \ldots \ldots \ldots$

4.16 TEM contrast for PFPA-FG (a), MCO-FG (b), and MBA-FG (c). Defocus values are $-0 \mathrm{~nm},-15 \mathrm{~nm},-45 \mathrm{~nm},-75 \mathrm{~nm}$ (from left to right), $\mathrm{U}=80 \mathrm{kV}, \mathrm{Cc}=1.3 \mathrm{~mm}, \mathrm{Cs}=1.2 \mathrm{~mm} \ldots \ldots \ldots$

5.1 Nearest neighbors in the coordination environment of the central carbon atom (A). Red dashed line corresponds the the atoms belonging to the same sublattice as (A); green dashed line corresponds to the atoms from the second sublattice $(\mathrm{B}) \ldots \ldots \ldots$

5.23 D representation (a) and a crossection through $\Gamma-K-M-\Gamma$ of graphene valence (blue) and conduction (red) bands . . . . . . . . .

5.3 Convergence of graphene total energy with respect to the density of the k-point mesh 
5.4 Relative variation of the total energy $\left(\frac{E_{t o t}-\left\langle E_{t o t}\right\rangle}{E_{t o t}}\right)$ in $\%$ as a function of the super cell size . . . . . . . . . . . . . . . .

5.5 Band structure (a) and Density of states (DoS) (b) calculated from the DFT methods. . . . . . . . . . . . . . . . . . . . . 96

5.6 Molecular orbital diagram of graphene . . . . . . . . . . . . 97

5.7 Wavefunctions corresponding to the first four bands in graphene . . 98

5.8 Valence electron density in graphene. (a) [100] orientation (the plane of atoms) (b) [001] orientation (the plane perpendicular to atoms) . 99

5.9 Wannier functions in graphene. . . . . . . . . . . . . . . . 100

5.10 Disentangled WF interpolated band structure of graphene. . . . . . 101

5.11 Band structure (a), ballistic transmission function (b), and density of states (c) of graphene in the $\Gamma-\mathrm{K}$ direction. . . . . . . . . 102

5.12 Band structure (a), ballistic transmission function (b), and density of states (c) of graphene in the $\Gamma$-M direction. . . . . . . . . . 102

5.13 Comparison between the number of eigenmodes (dashed) and the transmission function (solid) in the $\Gamma$-K direction in graphene. . . . 103

$5.14 \mathrm{I}-\mathrm{V}$ curves of graphene. In the inset: zoomed I-V curve in the the vicinity of zero bias . . . . . . . . . . . . . . . 104

5.15 Conductance curves for 10, 50, 100, and $400 \mathrm{k}$-points along the direction of conductance . . . . . . . . . . . . . . . . 104

5.16 Zigzag (a) and armchair (b) GNRs . . . . . . . . . . . . 106

5.17 Cuts along two directions of a graphene sheet, to produce zigzag (red) and armchair (green, blue) terminations of the GNRs . . . . . . 107

5.18 Folding of 2D graphene band structure for armchair (left) and zigzag (right) terminations. . . . . . . . . . . . . . . . . . 109

5.19 Strucutre of a 2zGNR. . . . . . . . . . . . . . . . . . . 110

5.20 Wannier functions in graphene from the periodic manifold . . . . 112

5.21 Wannier functions in graphene from the edge manifold . . . . . . 113

5.22 Band structure (a), ballistic transmission function (b), and density of states (c) of a 2zGNR . . . . . . . . . . . . . . . 114

5.23 Structure of a real GNR device . . . . . . . . . . . . . . 115

5.24 Band structure (a), ballistic transmission function (b), and density of states (c) of a structure in Fig. 5.23 . . . . . . . . . . . 115

5.25 Ballistic and coherent conductance for the structure in Fig. 5.23 . . 116

5.26 The phonon dispersion branches for a graphene sheet, plotted along high symmetry directions a) calculated using CASTEP b) calculated using GULP . . . . . . . . . . . . . . . . . . . . . . . 121

5.27 (a) Phonon dispersion relations, (b) Ballistic transmission function, and (c) Phonon density of states for the $\Gamma-\mathrm{K}$ (armchair) (left) and Г-M (zigzag) (right) directions in graphene . . . . . . . . . . . 122

5.28 Dispersion relations for zigzag GNRs . . . . . . . . . . . . . . . 124 
5.29 Shape functions and their Fourier transforms. a) Shape function for the armchair boundary, b) Shape function for the zigzag boundary c) Fourier transform of the shape function for the armchair boundary, d) Fourier transform of the shape function for the armchair boundary 130

5.30 Scaled attenuation coefficient $\frac{m W^{4}}{a^{3} L} t_{-n, m}$ as a function of k-vector: solid - from mode $\mathrm{m}=0$ to mode $-\mathrm{n}, \mathrm{n}=0 \ldots 2$, dashed - mode $\mathrm{m}=1$ to mode $-\mathrm{n}, \mathrm{n}=0 \ldots 2$, dashed-dotted $-\mathrm{m}=2$ to mode $-\mathrm{n}, \mathrm{n}=0 \ldots 2 . \quad \ldots 134$

5.31 Thermal conductivity as function of temperature . . . . . . . . 135

6.1 Fragment of the optimized structure of epoxy functionalized graphene (O-epoxygraphene). Carbon atoms are gray, oxygen -red. . . . . . . 142

6.2 Band structures of pristine (red) and epoxy-functionalized (blue) graphene. . . . . . . . . . . . . . . . . . 143

6.3 Wannier functions of S-epoxygraphene. Binding set . . . . . . . 146

6.4 Wannier functions of S-epoxygraphene. Nonbinding set . . . . . . 146

6.5 Band structure (a), conduction (b), and density of states (c) of epoxygraphene in the $\Gamma-\mathrm{K}$ (top row) and $\Gamma-\mathrm{M}$ (bottom row) directions. . 149

6.6 Bond lengths and hybridization angles in aminographene. . . . . . . 151

6.7 Band structures of pristine and amino-functionalized graphene . . . 152

6.8 Perfluorophenylazide molecule with methyl and "silane" moieties. . 153

6.9 PFPA attachment to a silicon substrate . . . . . . . . . . . 154

6.10 Structure of N-PFPA functionalized graphene. . . . . . . . . . . . 154

6.11 Fukui functions: $f^{+}$for a PFPA molecule (a), $f^{-}$for graphene (b). 155

6.12 Structure of C-PFPA functionalized graphene. . . . . . . . . . 156

6.13 Bond lengths and hybridization angles in PFPA-FG . . . . . . . 157

6.14 Band structures of pristine (red) and PFPA-functionalized (blue) graphene. Band structures were aligned with respect of the position of the Fermi level. . . . . . . . . . . . . . . . . . . . . . 159

6.15 Atomic structures of PFPA-FG . . . . . . . . . . . . . 162

6.16 Wannier functions of carbon atoms in N-PFPA-FG . . . . . . 163

6.17 Wannier functions of the heteroatom in N-PFPA-FG. . . . . . . . 163

6.18 Band structure, conductance, and density of states of B-, N-, P-, AsPFPA-FG in the $\Gamma-\mathrm{K}$ (top row) and $\Gamma-\mathrm{M}$ (bottom row) directions. . 165

6.19 Conductivity of PFPA-FG as a function of the heteroatom. . . . . . 166

6.20 Band structure (a), conduction (b), and density of states (c) of C-, Si- PFPA-FG in the $\Gamma-\mathrm{K}$ (left) and $\Gamma-\mathrm{M}$ (right) directions. . . . . 167

6.21 Comparison between theoretical (a) and experimental (b) conductance vs bias voltage curves. . . . . . . . . . . . . . . 168

$7.1 \quad$ Interaction of a $d$-metal with a carbonyl molecule. . . . . . . . 173

7.2 Structure of a Chromium(0)-bis-arene $\left(\mathrm{Cr}\left[\eta^{6}-\left(\mathrm{C}_{6} \mathrm{H}_{6}\right)_{2}\right]\right)$ molecule . 173

$7.3 \quad$ Structure of $\operatorname{Bis}\left(\eta^{6}\right.$-naphthalene $)$ chrome $(0) \ldots \ldots \ldots$ 
7.4 MO diagram of $\mathrm{Cr}\left(\mathrm{C}_{6} \mathrm{H}_{6}\right)_{2}$ (top) and interactions of the benzene orbital with the Cr orbitals of appropriate symmetry to form $\mathrm{Cr}\left(\mathrm{C}_{6} \mathrm{H}_{6}\right)_{2}$ (bottom).

7.5 Band structures of pristine (red) and functionalized (blue) graphene, computed with different degree of functionalization: one MA molecule per XxX graphene supercell. . . . . . . . . . . . . . . . . . 179

7.6 2x2 MAFG (a) initial geometry, top view and (b) optimized geometry, side view . . . . . . . . . . . . . . . . . . . . . 180

7.7 Ball-and-stick presentation of optimized structures of MA-functionalized graphene (top view) with one (a) and four (b) MA adducts per 6x6 graphene supercell. . . . . . . . . . . . . . . . . . . . . . . 180

7.8 Geometrical parameters of the MA-FG (a) M-C(arene) bond length, (b) M-C(graphene) bond length. . . . . . . . . . . . . . . . 182

7.9 Band structures of 1-MAFG and 4-MAFG, where $\mathrm{M}=\mathrm{Ti}, \mathrm{V}, \mathrm{Mn}, \mathrm{Fe}$. Red and blue coloring correspond to spin up and spin down bands. Energy reference level coincides with the position of Fermi level . . 186

7.10 Molecular orbitals (HOMO- top row, LUMO bottom row) for 1CrAFG (a), 4-CrAFG (b), 1-FeAFG (c), 4-FeAFG (d) . . . . . . 188

7.11 Wannier functions in $\mathrm{Cr}\left(\mathrm{C}_{6} \mathrm{H}_{6}\right)_{2} \ldots \ldots \ldots \ldots$

7.12 Wannier functions, centered on carbon atoms far away from the functionalizing metal atom (a), and in the direct vicinity of the metal atom (b) . . . . . . . . . . . . . . . . 190

7.13 BCD curves for MAGF with different degree of functionalization. . . 192

7.14 Conductivity of $\mathrm{Cr}-\mathrm{AFG} \ldots \ldots$

7.15 $\mathrm{BCD}$ in the $\Gamma-\mathrm{K}$ direction of $1-\mathrm{MAFG}$ and $4-\mathrm{MAFG}$, where $\mathrm{M}=\mathrm{Ti}$, $\mathrm{V}, \mathrm{Mn}(\mathrm{FM}), \mathrm{Fe} \ldots \ldots \ldots \ldots \ldots \ldots \ldots \ldots$

7.16 Spin resolved density of states in 4-VAFG (a) and 4-FeAFG (b) . . 196

7.17 Ferromagnetic (a) and antiferromagnetic (b) arrangement of spins of Mn atoms (purple rings) . . . . . . . . . . . . . 197

7.18 Wannier functions for 4Mn-MAFG, AFM $(\mathrm{a}, \mathrm{b})$ and FM $(\mathrm{c}, \mathrm{d})$ modifications . . . . . . . . . . . . . . . . . . 198

7.19 BCD for FM and AFM 4Mn-MAFG . . . . . . . . . . . 200

7.20 Structure of a Chromium(0)-monoarene-tricarbonyl $\left(\mathrm{Cr}\left[\eta^{6}-\left(\mathrm{C}_{6} \mathrm{H}_{6}\right)\right](\mathrm{CO})_{3}\right)$ molecule, an example of a "piano stool" . . . . . . . . . . . . 201

7.21 Different conformations of MCO molecules and their relative energies. 202

7.22 Interaction of a $d$-metal with a carbonyl molecule. . . . . . . . . . 203

7.23 Lone pair in MCO-FG . . . . . . . . . . . . . . . . . . . 203

7.24 MO diagram of $\mathrm{Cr}\left(\mathrm{C}_{6} \mathrm{H}_{6}\right)(\mathrm{CO})_{3}$ and interactions of the benzene orbital with the orbitals of the $\mathrm{Cr}(\mathrm{CO})_{3}$ fragment. . . . . . . . . 205

7.25 Band structures of pristine (red) and Piano stool-functionalized graphene(blue for closed shells or spin up and green for spin down). Band structures were aligned with respect of the position of the Fermi level. . . . . . 206 
7.26 Wannier functions in $\mathrm{Cr}\left(\mathrm{C}_{6} \mathrm{H}_{6}\right)(\mathrm{CO})_{3} \ldots \ldots \ldots 207$

7.27 BCD in $\mathrm{Cr}\left(\mathrm{C}_{6} \mathrm{H}_{6}\right)(\mathrm{CO})_{3}$ and $\mathrm{Cr}\left(\mathrm{C}_{6} \mathrm{H}_{6}\right)(\mathrm{CN})_{3} \ldots \ldots \ldots 209$ 


\section{LIST OF ABBREVIATIONS}

CNT Carbon Nanotube

GNR Graphene Nanoribbon

TEM Transmission Electron Microscopy

HRTEM High Resolution Transmission Election Microscopy

FFT Fast Fourier Transform

$\mathcal{F}$ Direct Fourier Transform

$\mathcal{F}^{-1}$ Inverse Fourier Transform

DFT Density Functional Theory

KS Kohn-Sham

XC Exchange-Correlation

LDA Local Density Approximation

GGA Generalized Gradient Approximation

SCF Self Consistent Field

TBA Tight-binding approximation

BZ Brillouin Zone

DoS Density of states

(ML)WF (Maximally localized) Wannier functions

NEGF Non-equilibrium Green's functions 
BCD Band structure, conductivity, DoS

xFG x-functionalized graphene

PFPA Perfluorophenyl azide

MA Metal arene

PS "Piano stool" (Metal(0)-tricarbonyl group)

AO Atomic orbitals

MO Molecular orbitals

HOMO/HOCO Highest occupied molecular/crystal orbital

LUMO/LUCO Highest occupied molecular/crystal orbital 
CHAPTER I

Introduction 
The aim of this research is to study the influence of the chemical modification of graphene surface on its structural and electronic properties. The dissertation's composition is based on the traditional material science tripod-schema: composition, structure, and properties. Although we limit ourselves to only one "composition" namely graphene, the number of possible doping molecules is infinite, so composition is understood in terms of the modifying molecule. Structural arrangement of the functionalizing group can also play crucial role in the perturbation of the electronic structure of pristine graphene. The properties section is related to calculation of readily experimentally observable properties along with the theoretical models that propose the quantum - mechanical mechanisms explaining the measurable effects.

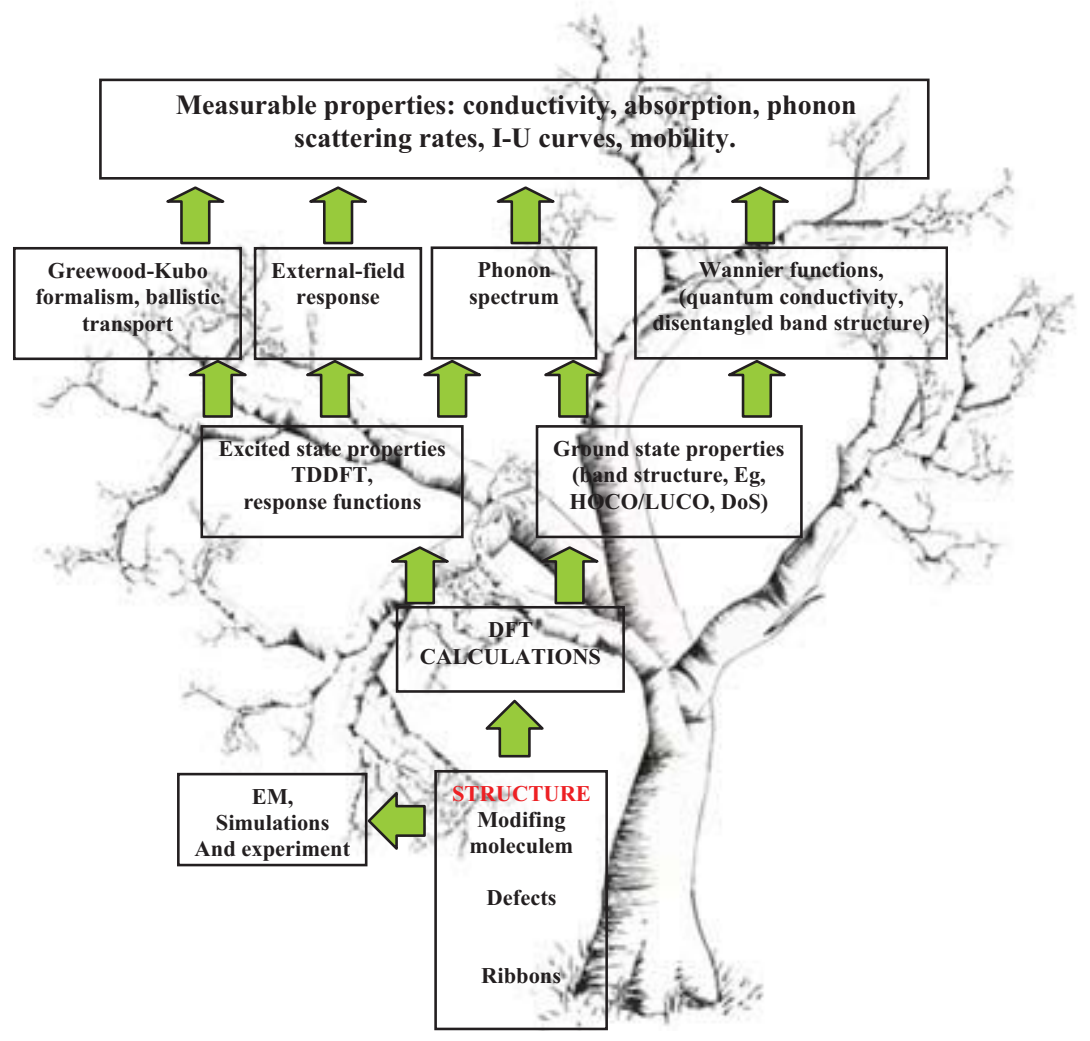

Figure 1.1: Schematic layout of the dissertation

Chapter I includes the motivation and introduction and literature review and also 
formulates the problem the dissertation addresses. Chapter II lays the foundation for the theoretical method used in this dissertation. Chapter III is focused on the foundations of quantum transport, and deals with the practical DFT calculations and interpretation of the results. In Chapter IV we first mention possible modifications of pristine graphene structure. This is the "trunk" and the lowest branch of the tree in Figure 1.1. In the very same chapter we discuss possible techniques of visual control of the structure of graphene, i.e. simulation of the high resolution transmission electron microscopy images of functionalized graphene and experimental observation of multiple layer graphene structures. Next three chapters are "material based", namely we undertake a study of graphene nanoribbons, covalently and hapticly functionalized graphene. Each of those chapters V, VI, and VII share the same structure: we begin with structural aspects of the material, present the results of the ground state calculations of the electronic properties within the DFT. Here we also undertake the analysis of the influence of the functionalizing molecules on both atomic and band structure. This includes the screening for the "best functionalizing molecule" in terms of desired trends in modification of graphene electronic structure. We will also analyze the bonding effects by means of the molecular orbital picture and construct the Wannier functions. These functions will be used to calculate quantum transport in the NEGF approximation. This dissertation is concluded by a summary of main results, proposed future work on functionalized graphene materials for electronics application, and a few technical notes. 


\subsection{Demand for fast microelectronic devices}

The progress in the microelectronics and computer science was aided by the wake of the progress in material science. The birth of modern microelectronics happened twice: first thanks to the theoretical works of Shockley and coworkers, who predicted a way to use the p-n junction to control strong signals by weak ones. That eventually lead to the invention of junction transistor. Second time was when the crystal growth process finally reached the stage at which the solid state transistors had reached and outreached the effectiveness of the vacuum tubes. The era of semiconductors began with the development of the techniques of manufacturing of bulky, almost defect free silicon wafers ${ }^{1}$. Silicon crystal grown by the floating zone and later the Czochralski method, exhibit very low concentration of dislocations and point defects $\left(10^{9}-10^{10} \mathrm{~cm}^{-3}\right)$. Further refinement of the growth approaches opened the door to production of the crystals about $400 \mathrm{~mm}$ in diameter and about $1.2 \mathrm{~m}$ long. The concentration of impurities in these crystals varies from 0.05 ppma for carbon to 49 ppmw for iron. Small number of defects creates very favorable condition for electronic transport: low scattering rate yields high mobility (the state of the art electron mobility in silicon is about $1400 \frac{\mathrm{cm}^{2}}{V \cdot s e c}$, compared to the 1998 top value $600 \frac{\mathrm{cm}^{2}}{V \cdot s e c}$ ) [1].

Typical "speed", i.e. the operation frequency of a single transistor (and thus of a circuit as whole) is governed by the characteristic relaxation time: $\tau=R \cdot C$, where $\mathrm{R}$ is the typical resistance of the transistor channel, and C - its capacity. Transforming from extensive variables to the intensive ones, we can write for the clock frequency $f \sim \frac{\mu}{\varepsilon}$. By the definition, dielectric permitivity $(\varepsilon)$ cannot be lower than 1 (Usually about 5 in semiconductors), whereas the electron mobility $(\mu)$ is theoretically limited

\footnotetext{
${ }^{1}$ We will not stop here on the early developments of semiconductors, like a cat's whisker detector, even though a very curios design of the diode and the fact that this technique has paved the way for modern portable radio receivers
} 
only be the mobility of ideal Fermi electron gas: 2,000,000 $\frac{\mathrm{cm}^{2}}{V \cdot s}$, i.e. the higher is the mobility the higher is the top operation frequency.

The demand for higher electron mobility eventually shifted the cutting edge paradigm from silicon to alternative semiconductors with higher mobility, such as gallium arsenide. This material in bulk possesses the electron mobility four times of this for silicon. Despite major problems associated with the growth aspects, such as evaporation of relatively volatile gallium and toxic arsenic, and the necessity to maintain strict 1:1 ratio of the elements, major progress has been achieved during last 10 years. A couple of companies, (Marubeni, TriQuint, Semiconductor Wafer) have successfully commercialized the manufacturing process of GaAs wafers.

However, electron mobility even twice as high as this for silicon still does not cover growing demand for high speed calculations. Initially computer speed was directly proportional to the number of transistors in a microchip.

This led to formulation of the Moore's law: "The number of transistors that can be placed inexpensively on an integrated circuit has doubled approximately every two years." [2] This trend which has continued for more than 50 years (see Figure $1.2)$, starts to bend off. Nowadays other limitations on computer speed, previously obscured by the trend to increase the number of the components, begin to kick in.

Increase in performance can be achieved either through increase of the number of transistors, or by increase of the operational speed of each transistor separately. The first way is conventionally adapted by modern CPU manufacturers, implementing various parallelizations techniques (like multithreading, multicore) The later way, in turn, can be achieved by means of different techniques. They all are targeting to decrease the relaxation time $\tau$ of transistor's channel. Below we summarize some modern trends of increasing a single transistor's performance: 


\section{CPU Transistor Counts 1971-2008 \& Moore's Law}

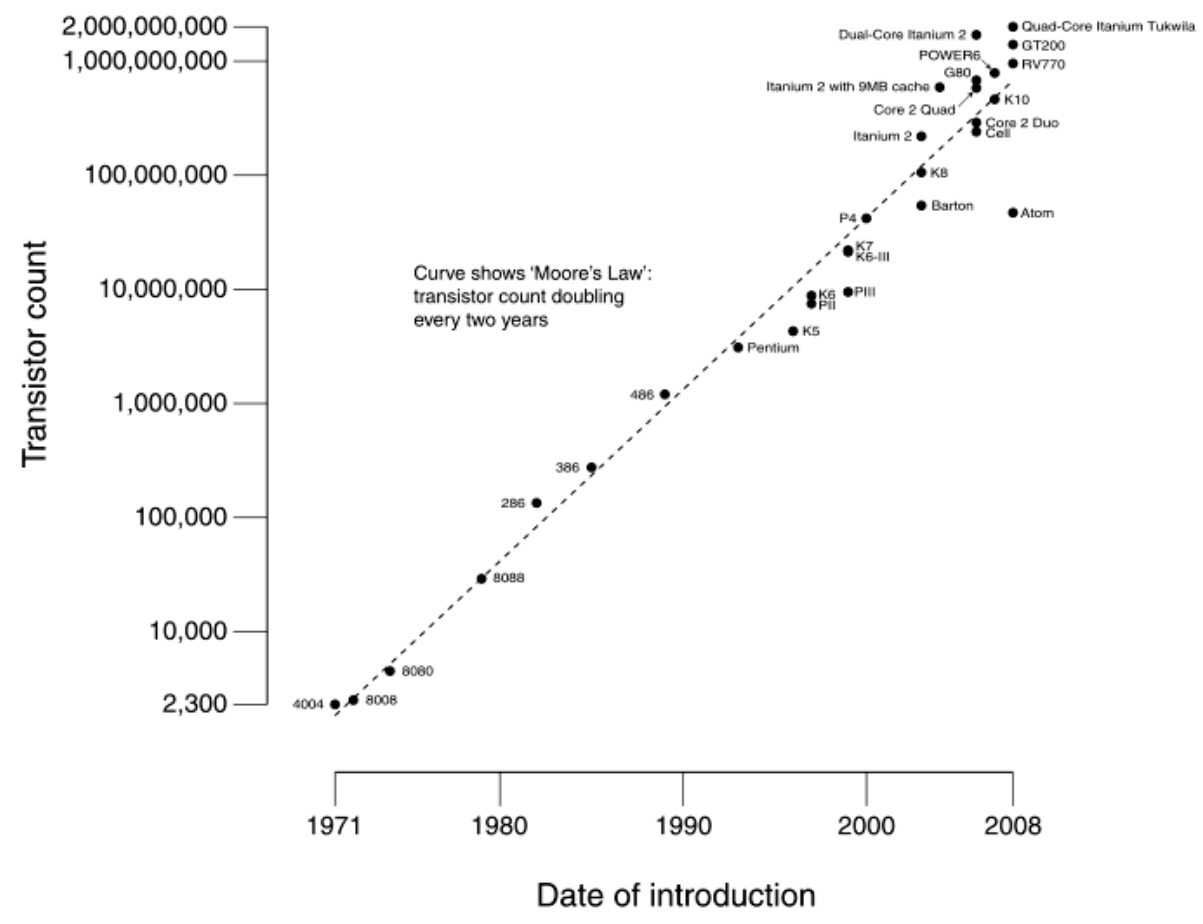

Figure 1.2: Plot of CPU transistor counts against dates of introduction. Adopted from $[2]$

- Cooling down the wafer. Low temperature decreases the number of occupied phonon states and thus suppresses the electron-phonon scattering. Thus gives increase in mobility that decreases the resistivity of the channel. (For example, researchers from IBM and Georgia Tech created a new speed record when they ran a silicon/germanium helium supercooled transistor at 500 gigahertz (GHz). The transistor operated above $500 \mathrm{GHz}$ at $4.5^{\circ} \mathrm{K}$ and simulations showed that it could likely run at $1 \mathrm{THz}[3]$.

- Decrease the size of the channel. This will lead to the drop of the capacitance of the channel, however increase electron scattering on the narrow borders of the channel. (In 2006, IBM announced that they had developed a technique to 
print circuitry only $29.9 \mathrm{~nm}$ wide using deep-ultraviolet (193-nanometer) optical lithography. IBM claims that this technique may allow chipmakers to use thencurrent methods for seven more years while continuing to achieve results forecast by Moore's law. New methods that can achieve smaller circuits are expected to be substantially more expensive.)

- "Nontraditional" and junction-less transistors. These methods usually utilize unusual properties of nanosized systems, such as quantum confinement. A transistor that consists of a control gate around a silicon nanowire that can tighten around the wire to the point of closing down the passage of electrons without the use of junctions or doping has been announced by the researchers at the Tyndall National Institute in Cork, Ireland The researchers claim that the new junctionless transistors can be produced at 10-nanometer scale using existing fabrication techniques [4].

The later approach has gained more attention relative to the others mentioned above, since it has wide perspectives for the development of a breakthrough technology in transistor manufacturing. One of the famous candidates for creating an industrially feasible junctionless transistor is the Carbon Nanotube (CNT). Depending on the chirality, they can exhibit either semiconductor or metallic behavior [5]. Two mutually connected CNTs of different chirality can produce an exceptionally fast Schottky diode [6]. The CNT also demonstrates exceptionally high mobility even at room temperature (up to $100,000 \frac{\mathrm{cm}^{2}}{V \cdot s e c}$ ). This exceptional performance eliminates the need for cooling down the wafer in order to archive maximal productivity. However, manufacturing a multiple CNT array of same properties still remains a challenging task. Carbon nanostructures have dominated in advanced nanoscience and nanotechnology since the last quarter of the 20th century. The 1996 Nobel Prize in Chemistry 
awarded jointly to Robert F. Curl, Jr., Sir Harold W. Kroto, and Richard E. Smalley "for their discovery of fullerenes" and the 2010 Nobel Prize in Physics awarded jointly to Andre Geim and Konstantin Novoselov "for groundbreaking experiments regarding the two-dimensional material graphene" recognize the potential impact of fullerenes, CNTs, graphene, and other carbon nanostructures in future nanotechnology-based discoveries. Structurally all carbon allotrope modifications (except diamond and lonsdaleite) share similar carbon atom networks, but exhibit significantly different properties that are dictated by size, shape, and chirality.

\subsection{The Rise of Graphene}

Graphene is one of many allotrope modifications of carbon among other dimensionally reduced structures such as fullerenes, carbon nanotubes and nanofibers, carbine and bulk diamond and lonsdailite. It consists of a single or two (graphene bi-layer possesses properties very similar to the single-layered graphene and is clearly distinctive from multilayered graphene) planar monoatomic sheets of $s p^{2}$ hybridized carbon atoms tightly packed into a two-dimensional (2D) honeycomb lattice which is the basic building block for graphitic materials of all other dimensionalities (Fig. 1.3). It can be wrapped up into 0D fullerenes, rolled into 1D nanotubes, or stacked into 3D graphite.

Theoretically graphene (or monolayer graphite) has been studied for the last sixty years: First theoretical studies of graphene were undertaken by Wallace in 1947 [8], and the first TEM observation was reported by Rüss and Vogt one year later [9]. Graphene's atomic structure is a natural simplification of graphite structure in which the interlayer van-der-Waals interaction is ignored. On the other hand, although known as an integral part of 3D materials, graphene was presumed not to exist in 

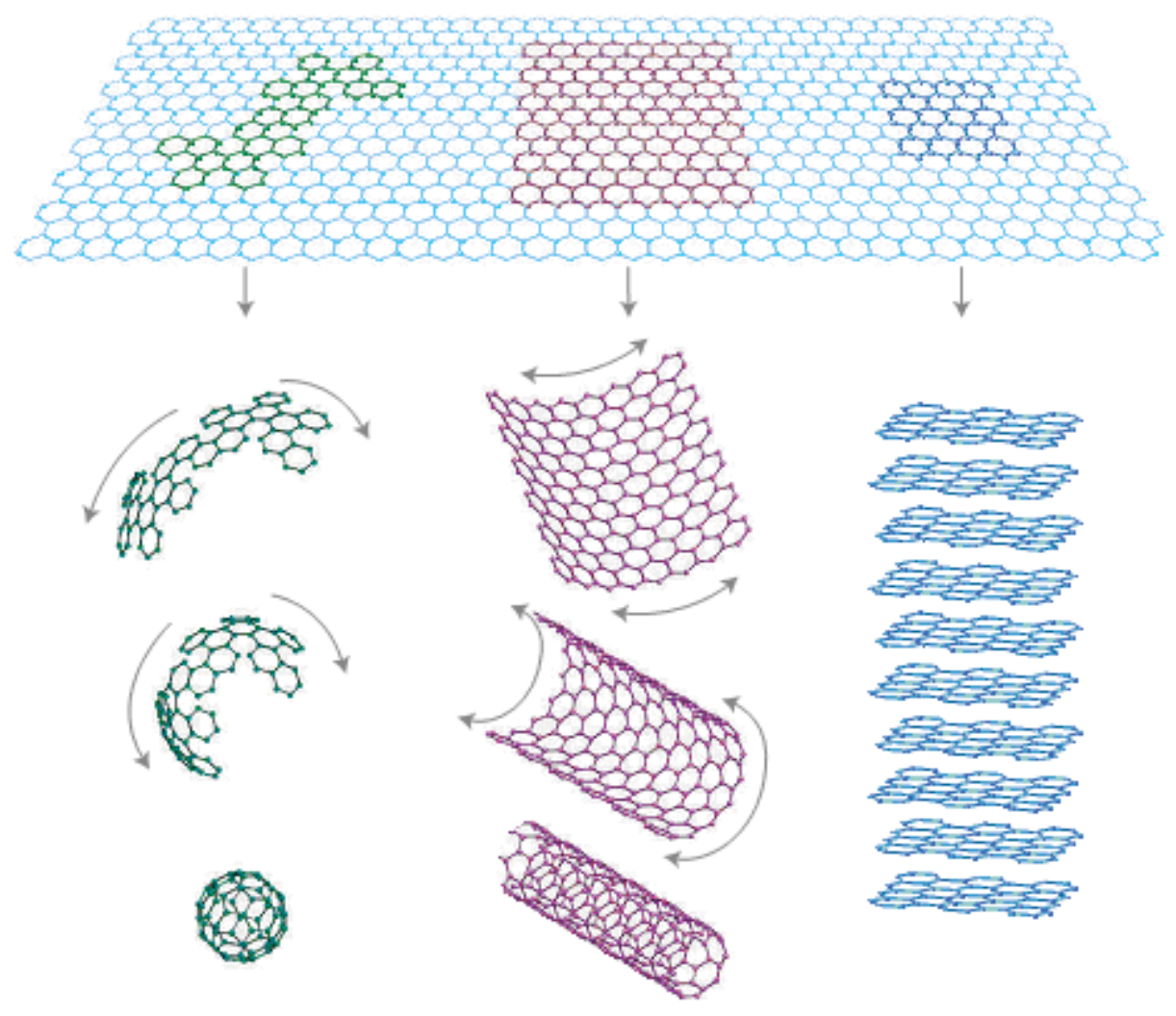

Figure 1.3: Graphene as a 2D building material for carbon materials of all other dimensionalities. It can be wrapped up into 0D buckyballs, rolled into 1D nanotubes or stacked into 3D graphite. Adopted from [7].

the free state, being described as an "academic" material [10] and was believed to be unstable with respect to the bucking, folding or formation of CNTs. More than 70 years ago, Landau and Peierls demonstrated that strictly 2D crystals are thermodynamically unstable and cannot exist [11]. From their theory it follows that a divergent contribution of thermal fluctuations in low-dimensional crystal lattices should lead to such displacements of atoms that they become comparable to interatomic distances at any finite temperature. Further studies confirmed this idea and imposed certain conditions on the type of interatiomic potential [12]. Therefore freestanding mono-atomic layers were believed to exist only as a part of a bulk 3D structures, usually grown 
epitaxially on top of monocrystals with matching crystal lattices (see e.g. a review by Evans et.al. [13]). Those films already demonstrate curious electronic properties due to the quantum confinement and thus possessing continuous electron spectrum in the lateral direction and the discreet one in the direction of growth. This confinement quantization gave rise to the whole family of heterostructural nanoelectronic devices. Because of their quasi-two dimensional nature, the effective mass of holes in the valence band is changed to more closely match that of electrons in the conduction band raising the overall carrier mobility in the device. By varying the composition of the solid solution one can tune the band gap over relatively broad ranges. These two factors, together with the reduced amount of active material in quantum wells, lead to their successful application in optical microdevices (laser diodes, infrared photodetectors, etc.) High carrier mobility found its application in the HEMTs (High Electron Mobility Transistors), which are crucial for the development of modern fast electronic integrated circuits. Certain doping techniques allow tweaking of the energy spectrum of electrons in the quantum well in such a way that it mimics that of a two-dimensional electron gas. Acceptor dopants can lead to a two-dimensional hole gas. Although there is a whole class of $2 \mathrm{D}$ materials that are readily available as quantum wells, a lot of experimental and theoretical efforts have so far been focused on graphene, shifting the point of interest towards its unique properties somehow ignoring the existence of other $2 \mathrm{D}$ crystals. A reason for that is a particular by unique nature of its charge carriers. In condensed matter physics, the stationary nonrelativistic Schrödinger equation is predominately used to describe electronic properties of materials. Graphene is an exception: its charge carriers mimic relativistic particles and are more easily and naturally described starting with the Dirac equation rather than the Schrödinger equation [14]. First E-k dispersion relation for graphene was obtained by Wallace [8] while he was trying to construct a simplified description 


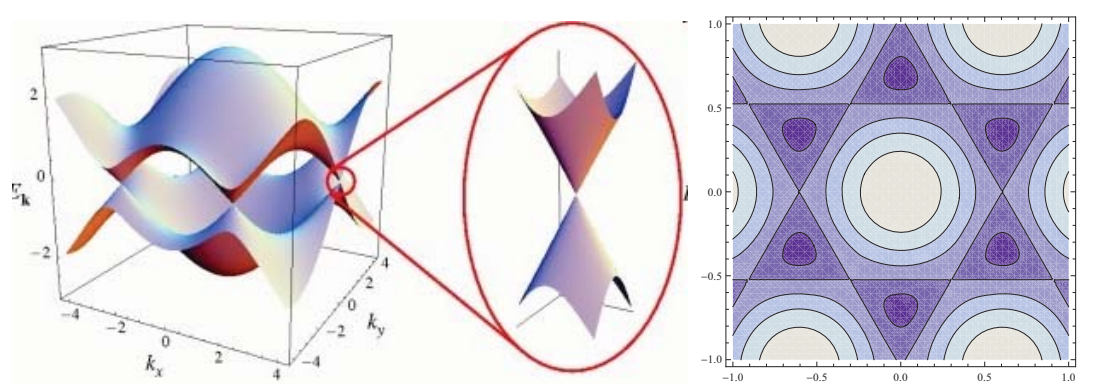

Figure 1.4: Dispersion relations in graphene. 3D view and Contour plot of the valence band

of band structure of graphite within the framework of the tight-binding approach. His studies revealed unusual conical E-k dispersion relationship at low energies close to the vertices of the of the two-dimensional hexagonal Brillouin zone, yielding zero effective mass for electrons and holes. Due to this linear (conical) dispersion relationship at low energies, electrons and holes near these six points, two of which are inequivalent, behave like relativistic particles described by the Dirac equation for spin 1/2 particles. Hence, the electrons and holes are called Dirac fermions, and the six corners of the Brillouin zone are called the Dirac points. The equation describing the E-k relation in the vicinity of the $\mathrm{k}$-point $\mathrm{K}$ is $E=\hbar v_{F} \sqrt{k_{x}^{2}+k_{y}^{2}}$, where the Fermi velocity $v_{F} \sim 10^{6} \mathrm{~m} / \mathrm{s}$. This dispersion relationship makes the effective mass of the charge carriers to be zero.

From the absence of the gap in the energy spectrum of graphene (Figure 1.4), it follows that in graphene charge carriers can be tuned continuously between electrons and holes in concentrations $n$ as high as $10^{13} \mathrm{~cm}^{-2}$ and their mobilities $\mu$ can exceed $15,000 \frac{\mathrm{cm}^{2}}{V \cdot s}$ even under ambient conditions $[14,15]$. Moreover, the observed mobilities weakly depend on the temperature, which means that $\mu$ at $300 \mathrm{~K}$ is still limited by impurity scattering, and therefore can be improved significantly, perhaps, even up to $\sim 100,000 \frac{\mathrm{cm}^{2}}{V \cdot s}$. Although some semiconductors exhibit room-temperature $\mu$ as high as $\sim 77,000 \frac{\mathrm{cm}^{2}}{V \cdot s}$ (namely, InSb), those values are quoted for undoped bulk semicon- 
ductors. In graphene, $\mu$ remains high even in highly $\left(n>10^{12} \mathrm{~cm}^{-2}\right)$ electrically and chemically doped devices [16], which translates into ballistic transport on the submicrometer scale (currently up to $\sim 0.3 \mu \mathrm{m}$ at $300 \mathrm{~K}$ ).

\subsection{Chemical doping of graphene}

Despite many evident advantages of graphene as a perspective material for nanoelectronic applications, is plagued by one severe handicap: absence of the energy gap. As it can already be seen from the Wallace solution [8], that the conduction band and the valence band touch each other at the k-point $K$. Pristine graphene devices can be exceedingly useful as conductors due to their high mobility and thus are low resistively, have high tolerable current density, and absence of electromigration. However, graphene is fairly useless as a switching device because of its semimetallic nature. Therefore, producing a band gap is probably one of the most important challenges that needs to be addressed before it can ultimately enable practical applications ranging from digital electronics to nanophotonics. A number of possible solutions have been proposed and demonstrated for producing bandgaps in single-and double-layered graphene. One of the more straightforward methods involves growth of epitaxial graphene on a lattice matched ( $\mathrm{SiC}$ ) substrate to induce a stress and, as a result, open up a bandgap of about $0.26 \mathrm{eV}$ [17]. One of the somewhat more successful methods utilizes quantum confinement to open a bandgap in graphene by producing nano-scale structures, i.e., quantum dots and nanoribbons where the bandgap varies inversely with the nanoscale structure dimension $[18,19,20]$. However, the non-uniform edges of these structures play a major role by degrading their electrical properties [21]. For example, with graphene nanoribbon of widths less than $10 \mathrm{~nm}$, a bandgap of about $0.4 \mathrm{eV}$ has been reported, but with an electron the mobility 
of about 100-200 $\frac{\mathrm{cm}^{2}}{V \cdot s}$. The mobility is believed to be degraded by edge scattering [22]. Chemical modification of graphene by functionalization of its surface with different molecules potentially allows a wider flexibility in engineering its electronic structure, in particular the local density of states of the carbon atoms bound to the modifier that can result in opening of the band gap. This application of graphene is the primary focus of this dissertation. Chemically functionalized graphene can also find applications in various areas beyond microelectonics [23]:

* The ability of graphene to adsorb hydrogen makes it an excellent candidate for hydrogen fuel storage [24, 25].

* Graphitic carbon is considered the state-of-the-art material for the negative electrode in lithium ion batteries. Modeling of lithium diffusion in graphene assists in the rational design of carbonaceous electrodes with high charge/discharge rates $[26]$.

* Graphene provides an ideal model for interaction of water with its hydrophobic surfaces [27]. Such interactions are very important in biological applications.

* By covalently functionalizing graphene with chromophores, porphyrin, or fullerene, it is possible to enhance non-linear optical performance in the nanosecond regime [28].

* Covalent functionalization of epitaxial graphene by azidotrimethylsilane has enabled tuning of its bandgap via $\pi-\pi$ interactions between porphyrin and graphene and has also been exploited to prepare graphene films with superior conductive properties $[29,30]$. 


\subsection{Motivation and overview}

One goal of this work is to evaluate the possibility of application of graphene-based materials in electronic devices. To achieve this goal, relevant physical properties of functionalized graphene were studied and compared to the aforementioned requirements for applications. Another goal is to investigate fundamental physical properties of different functionalizing groups used in graphene-based activated semiconductors and understand the origin of dependence of electric properties of the composition of the functionalizing molecules. This work also includes chapters that describe in more detail the methods used to complete this work: Transmission Electron Microscopy (TEM) and the Density Functional Theory (DFT)calculations, (Maximally localized) Wannier functions ((ML)WF) and NEGF based transport approach. Source codes of various self-written postprocessing MATLAB programs are available for the users at Portland State University. 


\section{CHAPTER II}

\section{The Density functional theory}


In this chapter we will describe the main approach used to calculate the electronic properties of graphene. A comprehensive analysis and computational aspects of the DFT theory can be found in "Electronic Structure Basic theory and practical methods" by R.Martin [31].

Quantum mechanics and electromagnetism are widely perceived as leading to a first-principles" approach to materials and nanosystems: if the needed soft-ware applications and corresponding hardware were available, their properties could be obtained without any adjustable parameter (nuclei characteristics being given). Still, such "first-principles" equations (e.g. N-body Schrödinger equation) are too complex to be handled directly. Indeed, fundamental quantities, like the wavefunctions for $\mathrm{N}$ particles cannot be represented faithfully on the hardware that is available nowadays for $\mathrm{N}$ bigger than about a dozen. Different methodologies to address this problem have been proposed. We will focus on the Density Functional Theory (DFT), proposed in 1964-65 by Hohenberg and Kohn [32], and Kohn and Sham [33], as well as the Many-Body Perturbation Theory, in particular the so-called GW approximation (GWA) of L. Hedin [34], proposed in 1965. Both significantly reduce the complexity of the first-principles approach at the expense of some approximations. In the eighties, on the basis of such methodologies it became clear that numerous properties of materials, like total energies, electronic structure, and dielectric, mechanical, magnetic, vibrational properties, can be obtained with an accuracy that can be considered as truly predictive.

\subsection{The Hohenberg-Kohn theorem}

The stating point for every quantum mechanical calculations is the Schrödinger

equation: $\hat{H} \Psi=E \Psi$, where $\hat{H}$ is the Hamiltonian of the system. Despite it simple 
look, the equation can be solved in the closed form for the simplest systems only. The reason for that is the nonlocality of the Hamiltonian. The Hamiltonian of a system of electrons and nuclei can be written as a sum of the following contributions:

$$
\begin{aligned}
& \hat{H}=\hat{T}+\hat{V}_{\text {ext }}+\hat{V}_{\text {int }}+E_{I I} \\
& \hat{T}=-\frac{\hbar^{2}}{2 m_{e}} \sum_{i} \nabla_{i}^{2} \\
& \hat{V}_{\text {ext }}=-\frac{1}{4 \pi \varepsilon_{0}} \sum_{i, I} \frac{Z_{I} e^{2}}{\left|r_{i}-\mathbf{R}_{I}\right|} \\
& \hat{V}_{\text {int }}=\frac{1}{8 \pi \varepsilon_{0}} \sum_{i \neq j} \frac{e^{2}}{\left|\mathbf{r}_{i}-\mathbf{r}_{j}\right|} \\
& E_{I I}=-\sum_{i} \frac{\hbar^{2}}{2 M_{i}} \nabla_{I}^{2}+\frac{1}{8 \pi \varepsilon_{0}} \sum_{I \neq J} \frac{Z_{I} Z_{J} e^{2}}{\left|\mathbf{R}_{i}-\mathbf{R}_{j}\right|}
\end{aligned}
$$

Where $T$ is the kinetic energy operator for the electrons, $V_{\text {ext }}$ is the potential acting on the electrons due to the nuclei, $V_{\text {int }}$ is electron-electron interaction, and the final term $E_{I I}$ is the classical interaction of nuclei with one another and their kinetic energy. Electrons are denoted by lowercase subscripts, nuclei by uppercase subscripts. Since the atoms are more than 2000 times heavier than the electrons, their kinetic energy is much smaller than the one of the electrons. Thus we can separate the variables, assuming that the electrons immediately react on any change in the nuclear configuration. This assumption is called the adiabatic approximation. The total wave function of the system can now be factorized on the electron wave function and the nuclear wave function. Nuclear movement is important for the phonon related properties (such as heat capacity, heat transports, etc) whereas electron movement is responsible for the chemical bonding and the electronic properties (such as band gap, absorption spectrum, etc.) As long as we are not interested in electron-phonon interaction, we can assume that the atoms do not move at all. This assumption 
is called the Born-Oppenheimer approximation. Under this approximation the first summation in the $E_{I I}$ term vanishes (since the nuclei do not move any more, their kinetic energy is zero) and the last term reduces to a constant. We include this constant and all other external perturbations into $V_{\text {ext }}$. Using the adiabatic and the Born Oppenheimer approximations and adopting Hartree atomic units: $\hbar=m_{e}=$ $e=\frac{1}{4 \pi \varepsilon_{0}}=1$, Eq. (2.1) for electrons can be written as:

$$
\begin{aligned}
& \hat{H}=-\frac{1}{2} \sum_{i} \nabla_{i}^{2}+\frac{1}{2} \sum_{i \neq j} \frac{1}{\left|\mathbf{r}_{i}-\mathbf{r}_{j}\right|}+\left(\sum_{i, I} \frac{Z_{I}}{\left|r_{i}-\mathbf{R}_{I}\right|}+\frac{1}{2} \sum_{I \neq J} \frac{Z_{I} Z_{J}}{\left|\mathbf{R}_{i}-\mathbf{R}_{j}\right|}\right) \\
& \hat{H}=\hat{T}+\hat{V}_{\mathrm{int}}+\hat{V}_{e x t}
\end{aligned}
$$

This Hamiltonian in combination with the Schrödinger equation contains full information about quantum mechanical behavior of the system. Even though Eq. (2.2) is much simpler than the original Eq. (2.1), it is still too difficult to solve. One of a very powerful methods to solve the Schrödinger equation with the Eq. (2.2) Hamiltonian is the Density Functional Theory (DFT). The essence of the DFT framework consists of two theorems:

1. There is a one-to-one correspondence between the ground state electron density $\rho(r)$ of a many electron system and the external potential $V_{\text {ext }}$. Thus the eigenvalue of any observable operator is a unique functional of the ground state electron density.

2. If this observable operator is chosen to be the Hamiltonian, than the ground state total energy functional at the given $V_{e x t}$ is of the form:

$$
E_{V_{\text {ext }}}[\rho]=\left\langle\Psi\left|\hat{T}+\hat{V}_{\text {int }}\right| \Psi\right\rangle+\left\langle\Psi\left|\hat{V}_{\text {ext }}\right| \Psi\right\rangle=F_{H K}[\rho]+\int \rho(\mathbf{r}) V_{\text {ext }}(\mathbf{r}) d \mathbf{r}^{1}
$$

${ }^{1}$ Equation (2.3) can be written as the eigenequation of the density operator: $\hat{\rho}(\mathbf{r})=\sum_{i=1}^{N} \delta\left(\mathbf{r}_{i}-\mathbf{r}\right)$, 
The Hohenberg-Kohn density functional $F_{H K}[\rho]$ is same for every many electron system, and $E_{V_{e x t}}[\rho]$ reaches its minimal value (the ground state energy) when $\rho$ reaches the ground state electron density for the given $V_{\text {ext }}$. Those two theorems provide a great computational advantage: instead of dealing with a function of $3 \mathrm{~N}$ variables ( $\mathrm{N}$ is the number of electrons in the system), we can use a function of three variable. The density contains as much information as the wavefunction does; therefore all observable quantities can be obtained form the density only. For the proof of this absolutely nonintuitive theorem Hohenberg and Kohn received the Nobel Prize in 1963 .

\subsection{The Kohn-Sham equations}

The Hohenberg-Kohn density functional $F_{H K}[\rho]$ is universal, i.e. does not depend on any given atomic configuration, even though that its exact closed form mathematical expression is not know in any case, its contribution to the total energy can be easily calculated once the ground-state density is found. The Rayleigh-Ritz variational principle can be used to find the ground state density. Out of the infinite number of possible electron densities the one that minimizes $E_{V_{e x t}}[\rho]$ is the ground-state density. However, in order to do so we have to know the $F_{H K}[\rho]$ or an approximation to it. where $\mathrm{N}$ in the number of particles in the system. Thus the many body density is given by:

$$
\begin{aligned}
& \rho(\mathbf{r})=\left\langle\Psi\left(\mathbf{r}_{1}, \mathbf{r}_{2}, \ldots, \mathbf{r}_{N}\right)|\hat{\rho}(\mathbf{r})| \Psi\left(\mathbf{r}_{1}, \mathbf{r}_{2}, \ldots, \mathbf{r}_{N}\right)\right\rangle=\left\langle\Psi\left(\mathbf{r}_{1}, \mathbf{r}_{2}, \ldots, \mathbf{r}_{N}\right)\left|\sum_{i=1}^{N} \delta\left(\mathbf{r}-\mathbf{r}_{i}\right)\right| \Psi\left(\mathbf{r}_{1}, \mathbf{r}_{2}, \ldots, \mathbf{r}_{N}\right)\right\rangle= \\
& \sum_{i=1}^{N} \int\left|\Psi\left(\mathbf{r}_{1}, \mathbf{r}_{2}, \ldots, \mathbf{r}_{i}=\mathbf{r}, \ldots, \mathbf{r}_{N}\right)\right|^{2} d \mathbf{r}_{1} d \mathbf{r}_{2} \ldots d \mathbf{r}_{i} \ldots d \mathbf{r}_{N}
\end{aligned}
$$


The Hohenberg-Kohn functional can be rewritten as:

$$
\begin{aligned}
& F_{H K}[\rho]=\left\langle\Psi\left|\hat{T}+\hat{V}_{i n t}\right| \Psi\right\rangle=T+V \\
& =T+V-T_{0}+T_{0}=T_{0}+V+\underbrace{\left(T-T_{0}\right)}_{V_{c}} \\
& =T_{0}+V+V_{c}+V_{H}-V_{H}=T_{0}+V_{H}+\underbrace{\left(V-V_{H}\right)}_{V_{x}} \\
& =T_{0}+V_{H}+\underbrace{\left(V_{x}+V_{c}\right)}_{V_{x c}}=T_{0}+V_{H}+V_{x c}
\end{aligned}
$$

Here $T$ and $V$ are exact kinetic and electron-electron potential functionals. $T_{0}$ and $V_{H}$ are the kinetic energy functional for noninteracting electrons and the Hartree energy, respectively. The correlation energy $V_{c}$ is the part of the total energy that is responsible for the electron-electron interaction, thus we can define it as the difference in kinetic energies for the interacting and non-interacting electron gases: $V_{c}=T-T_{0}$. The exchange contribution to the total energy is defined as the part which is present in the exact solution, but absent in the Hartree solution. It can be defined as $V_{x}=$ $V-V_{H}$. This partitioning scheme is especially useful since the only formally unknown term contains the difficult exchange and correlation contributions only. The energy functional can be explicitly written as: $E_{V_{e x t}}[\rho]=T_{0}[\rho]+V_{H}[\rho]+V_{x c}[\rho]+V_{e x t}[\rho]$ and the corresponding total Hamiltonian (the Kohn-Sham Hamiltonian) can be rewritten as:

$$
\hat{H}_{K S}=\hat{T}_{0}+\hat{V}_{H}+\hat{V}_{x c}+\hat{V}_{e x t}=-\frac{\hbar^{2}}{2 m_{e}} \nabla^{2}+\frac{e^{2}}{4 \pi \varepsilon_{0}} \int \frac{\rho\left(\mathbf{r}^{\prime}\right)}{\left|\mathbf{r}-\mathbf{r}^{\prime}\right|} d \mathbf{r}^{\prime}+V_{x c}[\rho]+V_{e x t}[\rho]
$$


Thus, the ground state electron density of an N-electron system can be obtained as a solution of a Schrödinger-like (Kohn-Sham) single particle equation as:

$$
\begin{aligned}
& \rho(\mathbf{r})=\sum_{i=1}^{N} \phi_{i}(\mathbf{r}) \phi_{i}^{*}(\mathbf{r}) \\
& \hat{H}_{K S}[\rho] \phi_{i}(\mathbf{r})=\varepsilon_{i} \phi_{i}(\mathbf{r})
\end{aligned}
$$

The single particle wave functions $\phi_{i}(r)$ are not the electron wavefunctions, in general they have no physical meaning, only the total density as obtained from Eq. (2.6) is same as the true total density obtained from the density operator (see the footnote in page 18). Also single particle energies $\varepsilon_{i}$ are not single-electron energies. Both the Hartree operator and the Exchange-Correlation (XC) operator depend on electron density $\rho(r)$, which in turn, depend on the $\phi_{i}(r)$ which are obtained as the solution of the Kohn-Sham equation. The solution of the Kohn-Sham equation is an iterative process: We start from the trial electron density $\rho_{0}$, usually just a linear combination of atomic densities, calculate the $V_{H}$ and $V_{x c}$, obtain $\hat{H}_{K S}$, solve the eigenvalue problem, that results in a set of $\phi_{0}$ from which a density $\rho_{0}$ can be derived, etc. This cycle is stopped when the convergence criterion is reached: either the variation of the electron density, or the Kohn-Sham energies get below certain threshold. This can be represented as a self-consisted scheme

$$
\begin{aligned}
& \rho_{0} \rightarrow V_{H}^{i}, V_{x c}^{i} \rightarrow H_{H S}^{i} \\
& \uparrow \downarrow \\
& \rho_{\text {fin }} \leftarrow \rho^{i} \leftarrow \phi^{i}
\end{aligned}
$$

It is important to note that the Kohn-Sham scheme is exact; no other approximation except the Born-Oppenheimer was made so far. However, the exact mathematical 
expression of the $V_{x c}$ is unknown. The form of the $\mathrm{XC}$ functional has been a subject of intense research trying to construct a functional that would describe electron-electron interaction beyond the Hartree approach.

\subsection{Exchange-Correlation functionals}

Many different functionals of different level of approximation and different applicability have been proposed, where a general expression for the XC energy takes the form:

$$
E_{x c}[\rho]=\int \rho(\mathbf{r}) \varepsilon_{x c}[\rho(\mathbf{r})] d \mathbf{r}
$$

Here $\varepsilon_{x c}[\rho(r)]$ may now be both a function of $r$ or a functional depending on the level of approximation. The Exchange-Correlation potential in Eq. (2.6) is by the definition: $V_{x c}[\rho]=\frac{\delta E_{x c}[\rho]}{\delta \rho}$, thus if $E_{x c}[\rho]$ has the form of Eq. (2.8), then $V_{x c}[\rho]=$ $\varepsilon_{x c}[\rho]+\rho \frac{\delta \varepsilon_{x c}[\rho]}{\delta \rho}$.

A widely used simple approximation is the Local Density Approximation (LDA) that postulates that every infinitesimal volume is filled with a homogenous electron gas (HEG) of different density, which has the same total density as the overall density of the original material. In this approximation the Exchange-Correlation energy $E_{x c}$ takes the form: $E_{x c}[\rho]=\int \rho(\mathbf{r}) \varepsilon_{x c}(\rho(\mathbf{r})) d \mathbf{r}$ (note the function, not functional, i.e. dependence of the expression for $E_{x c}$ on $\rho(\mathbf{r})$ only, not any implicit variables). The exact form of $E_{x}(\rho(r))$ for HEG is

$$
E_{x}^{\mathrm{LDA}}[\rho]=-\frac{3}{4}\left(\frac{3}{\pi}\right)^{1 / 3} \int \rho(\mathbf{r})^{4 / 3} \mathrm{~d} \mathbf{r}
$$

Analytic expressions for the correlation energy of the HEG are not known except in the high- and low-density limits corresponding to infinitely-weak and infinitely-strong 
correlation. The most popular LDAs for the correlation energy density interpolate these accurate values obtained from simulation while reproducing the exactly known limiting behavior. Various approaches, using different analytic forms for $\varepsilon_{c}$, have generated several correlation functionals for the LDA, including Vosko-Wilk-Nusair (VWN) [35]; Perdew-Zunger (PZ81) [36]; Perdew-Wang (PW92) [37].

The electron density of the neighboring cells can be incorporated into the calculation by making the $\varepsilon_{x c}(\rho(r))$ be also a function of the electron density gradient, i.e. $\left.\varepsilon_{x c}(\rho(r)), \nabla \rho(r)\right)$. This gives rise to the family of Generalized Gradient Approximation (GGA). Using the GGA very good results for molecular geometries and ground-state energies have been achieved. Most known GGA functionals are PW91 [38] and PBE [39], (both of which have as an ingredient the LDA correlation. , and a choice needs to be made between VWN, PZ, and PW for exchange) and BLYP $[40,41]$ with Becke88 [42] for exchange and the Lee-Yang-Parr [41] for correlation.

Unfortunately, both LDA and GGA are plagued by the so-called "band gap problem"; they predict band gaps in bulk semiconductors that are way too small. Typical error in the position of energy bands varies from 5 to 20\%. Majewski et.al. [43] discussed the influence of the usage of exact exchange (EXX) versus the LDA. (See Fig. 2.1).

The Generalized Gradient Approximation (GGA) goes somewhat further including into the equation for the exchange correlation functional the terms dependent on the gradient of electron density, i.e $\epsilon_{x c}(\rho(r), \nabla \rho(r))$. (see Eq. (2.8)). The GGA however, significantly overestimates chemical binding energy, resulting in shortening of bonds during geometry optimization. One remedy is to correct LDA and GGA calculations - a posteriori by calculating quasiparticle self-energies in terms of the so-called GW method [34]. This approach extends the mathematical formulation form Eq. (2.8) to 


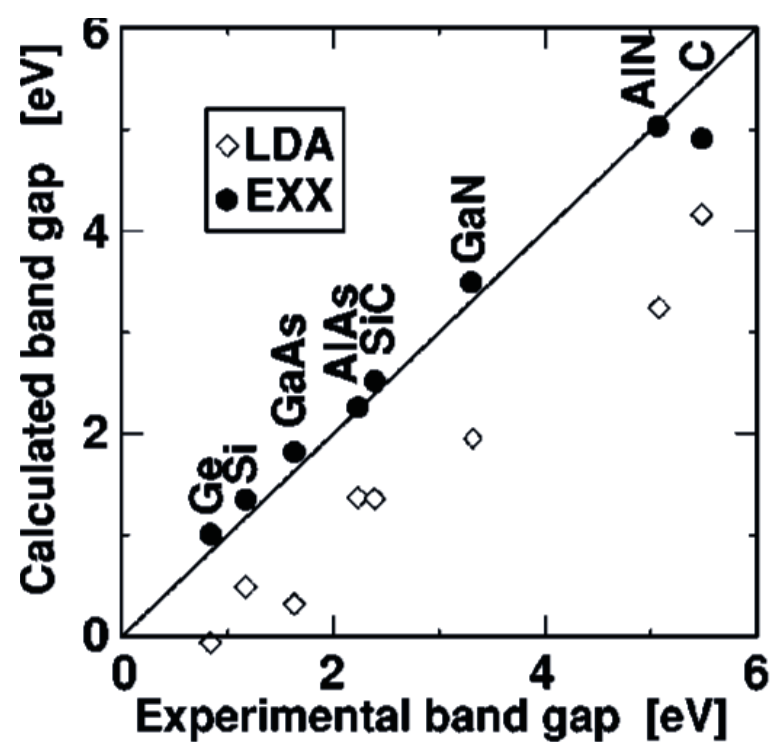

Figure 2.1: Comparison of self-consistently calculated LDA and EXX band gaps (in $\mathrm{eV}$ ) of various semiconductors with experimental data. Adopted from [43]

an integral operator of the wave function with the self-energy $(\Sigma)$ kernel:

$$
\begin{aligned}
& {\left[\hat{T}_{0}+\hat{V}_{H}+\hat{V}_{e x t}\right] \phi_{i}(\mathbf{r})+\hat{V}_{x c} \phi_{i}(\mathbf{r})=\varepsilon_{i} \phi_{i}(\mathbf{r})} \\
& {\left[\hat{T}_{0}+\hat{V}_{H}+\hat{V}_{e x t}\right] \psi_{i}(\mathbf{r})+\int \Sigma\left(\mathbf{r}, \mathbf{r}^{\prime} ; E_{i}\right) \psi_{i}\left(\mathbf{r}^{\prime}\right) d \mathbf{r}^{\prime}=E_{i} \psi_{i}(\mathbf{r})}
\end{aligned}
$$

In this approximation $E_{i}$ are exact quasiparticle energies, i.e. have clear physical meaning unlike the Kohn-Sham eigenvalues $\varepsilon_{i}$. Although the method is very physically transparent and precise, it bears one handicap - instead of one Self Consistent Field (SCF) cycle, it contains multiple nested cycles required for calculation of each quasiparticle self-energy. Therefore it is hardly suitable for anything but a very small unit cell, like pristine graphene.

Recently the hybrid functionals have drawn wide attention; they are mostly free from birth defects of the LDA and GGA. Hybrids incorporate a portion of exact 
exchange from the Hartree-Fock theory:

$$
E_{x}^{H F}=-\frac{1}{2} \sum_{i, j} \int \frac{\phi_{i}\left(\mathbf{r}_{1}\right) \phi_{j}^{*}\left(\mathbf{r}_{2}\right) \phi_{j}\left(\mathbf{r}_{1}\right) \phi_{i}^{*}\left(\mathbf{r}_{2}\right)}{\left|\mathbf{r}_{1}-\mathbf{r}_{2}\right|} d \mathbf{r}_{1} d \mathbf{r}_{2}
$$

(Here $\phi$ are the Kohn-Sham single particle orbitals) with exchange and correlation from the LDA or the GGA. The exact exchange energy functional is expressed in terms of the Kohn-Sham orbitals $\left(\phi_{i}(r)\right)$ rather than the density, so is termed an implicit density functional. Probably the most known and apparently the best so far within the assumption that the $E_{x c}$ is expressed as Eq. (2.8) is B3LYP [44, 45]

$$
V_{x c}^{\mathrm{B} 3 \mathrm{LYP}}=V_{x c}^{\mathrm{LDA}}+a_{0}\left(V_{x}^{\mathrm{HF}}-V_{x}^{\mathrm{LDA}}\right)+a_{x}\left(V_{x}^{\mathrm{GGA}}-V_{x}^{\mathrm{LDA}}\right)+a_{c}\left(V_{c}^{\mathrm{GGA}}-V_{c}^{\mathrm{LDA}}\right)
$$

Where $a_{0}=0.20, a_{x}=0.72$, and $a_{c}=0.81$ are the empirical parameters determined by fitting the predicted values to a set of atomization energies, ionization potentials, proton affinities, and total atomic energies, and $V_{x}^{G G A}$ and $V_{c}^{G G A}$ are the Becke88 exchange functional [42] and the correlation functional of Lee, Yang and Parr [41], and $V_{c}^{L D A}$ is the VWN local-density approximation to the correlation functional.

The LDA functionals show pretty good agreement with the experimental results for the geometrical parameters. The LDA is a remarkably good approximation in bulk crystals and its error for volumes (5\%) compares favorably to that produced by GGA functionals (4-8\%) and meta-GGA functionals (3-8\%). In molecular systems the highly parameterized meta-GGA and the hybrid functional are somewhat more accurate than the PBE GGA functional. Atomization energies and band structure calculated using the LDA functional have a tendency to overbind by $20-30 \%$. The GGA functionals yield very significant improvements with relative errors in the range 3-7\% and an average absolute error of $17 \mathrm{kcal} / \mathrm{mol}$. The highly parameterized B3LYP 
functional performs somewhat better than the BLYP and PBE functionals. The meta-GGA functionals yield relative errors of 2-3\% and average absolute errors of 3-5 $\mathrm{kcal} / \mathrm{mol}$.

\subsection{Solving the Kohn-Sham equations}

Eigenfunctions of the Kohn-Sham equations (2.6) with the Hamiltonian formulated in Eq. (2.5) can be represented as a serial expansion in a given basis set $\{\chi\}$

as $\phi_{i}=\sum_{\mu=1}^{N} c_{\mu}^{i} \chi_{\mu}$. The atomic orbitals $\chi_{\mu}$ are called the atomic basis functions and the $c_{\mu}^{i}$ are the MO expansion coefficients. Several choices are possible for the basis set, including Gaussian functions [46], Slater functions [47] plane waves [48, 49] and numerical orbitals. The wave functions $\phi_{i}$ belong to a function space which has an infinite dimension, $\mathrm{N}$ is therefore, in principle, infinite. In practice one works with a limited set of basis functions. Such a limited basis will never be able to describe $\phi_{i}$ exactly, but one could try to find a basis that can generate a function that is approximates $\phi_{i}$ better than any given in advance accuracy. Expansion coefficients $c_{j}^{i}$ can be found as the solution of the eigenequation:

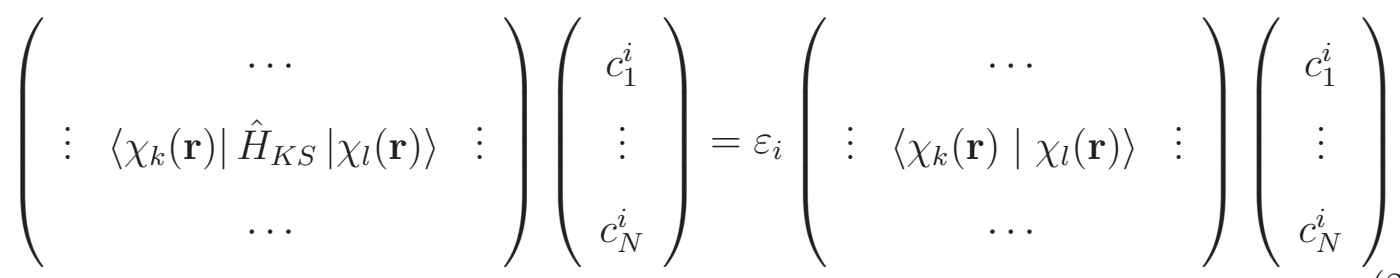

The matrix on the left is called the Hamiltonian matrix, and the matrix on the right - overlap matrix. If the basis is orthogonal, the overlap matrix is a unit matrix. $\mathrm{DMol}^{3}$ uses numerical orbitals for the basis functions $\left\{\chi_{\mu}(r)\right\}$, each function corresponding to an atomic orbital (AO). The basis functions $\chi_{\mu}$ are given numerically as values on an atomic-centered spherical-polar mesh, rather than as analytical 
functions (i.e. Gaussian orbitals). The angular portion of each function is the appropriate spherical harmonic $Y_{l m}(\theta, \varphi)$. The radial portion $F(\mathbf{r})$ is obtained by solving the atomic DFT equations numerically. A reasonable level of accuracy is usually obtained by using about 300 radial points from the nucleus to an outer distance of 10

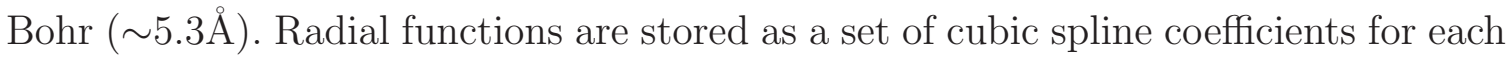
of the 300 sections, so that $F(r)$ is actually piecewise analytic. This is an important consideration for generating analytic energy gradients. In addition to the basis sets, the $-\nabla^{2} / 2$ terms required for evaluation of the kinetic energy are also stored as spline coefficients. Atomic basis sets are confined within a cutoff value, $r_{c}$, appropriate for a particular quality level of $\mathrm{DMol}^{3}$ calculations.

This is an important feature of the numerical basis set that can lead to much faster calculations, particularly for the systems with thick empty space between the atoms (layers of atoms). $\mathrm{DMol}^{3}$ uses soft confinement potential, which ensures the strict localization of the basis set within an $r_{c}$ value, without discontinuous derivatives at $r_{c}$. Therefore, unlike the plane wave basis set, there is no need to keep a set of high order basis functions, so that their superposition should vanish outside the atomic spheres. Geometry optimization is efficient, even with small cutoff values. The use of the exact DFT spherical atomic orbitals has several advantages. For one, the molecule can be dissociated exactly to its constituent atoms (within the DFT context). Because of the quality of these orbitals, basis set superposition effects [50] are minimized and it is possible to obtain an excellent description, even for weak bonds.

$\mathrm{DMol}^{3}$ is flexible in selection of the basis set: Generation of an entire second set of functions results in doubling the basis set size. This is referred to as a doublenumerical (DN) set. This notation is used by analogy with Gaussian double-zeta (DZ) sets, but the $\mathrm{N}$ is used to emphasize the numerical nature of these orbitals. For first-row atoms, functions from +2 ions provide a reasonable double basis set. A 
hydrogenic 3D orbital obtained for a nucleus of $\mathrm{Z}=5$ provides a good polarization function for each of these atoms. A hydrogenic $2 p$ function for $\mathrm{Z}=1.3$ is used for hydrogen. The use of various nuclear charges to generate polarization functions is analogous to the variation of zeta in Gaussian basis sets. For metals, $4 p$ polarization functions are generated by solving the atomic equations for a $4 s \longrightarrow 4 p$ excited state. Basis set quality has been analyzed in detail by Delley [50]. The table below summarizes the basis sets used in the program.

Table 2.1: Basis sets available in $\mathrm{DMol}^{3}$

\begin{tabular}{|c|c|c|}
\hline $\begin{array}{l}\text { Basis } \\
\text { Name }\end{array}$ & Description & Examples \\
\hline MIN & $\begin{array}{l}\text { Minimal basis. One AO for each oc- } \\
\text { cupied atomic orbital. Yields low ac- } \\
\text { curacy but fast computation. }\end{array}$ & $\begin{array}{l}\text { H: } 1 \mathrm{~s} \\
\text { C: } 1 \mathrm{~s} 2 \mathrm{~s} 2 \mathrm{p} \\
\text { Si: } 1 \mathrm{~s} 2 \mathrm{~s} 2 \mathrm{p} 3 \mathrm{~s} 3 \mathrm{p}\end{array}$ \\
\hline DN & $\begin{array}{l}\text { Double Numerical. MIN }+ \text { a second } \\
\text { set of valence AOs. Improved accu- } \\
\text { racy over MIN. }\end{array}$ & $\begin{array}{l}\text { H: 1s 1s' } \\
\text { C: 1s 2s 2p 2s' 2p' } \\
\text { Si: } 1 \mathrm{~s} 2 \mathrm{~s} 2 \mathrm{p} 3 \mathrm{~s} 3 \mathrm{p} 3 \mathrm{~s}^{\prime} 3 \mathrm{p}^{\prime}\end{array}$ \\
\hline DND & $\begin{array}{l}\text { Double Numerical plus d-functions. } \\
\text { Like DN with a polarization d- } \\
\text { function on all non-hydrogen atoms. } \\
\text { The default basis set, providing rea- } \\
\text { sonable accuracy for modest compu- } \\
\text { tational cost. }\end{array}$ & 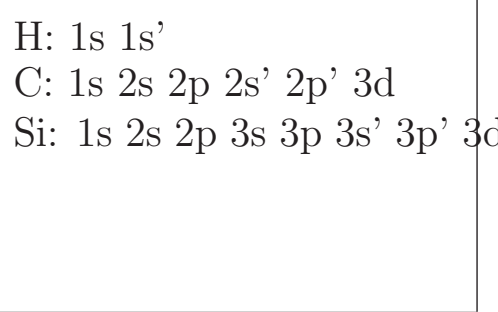 \\
\hline DNP & $\begin{array}{l}\text { Double Numerical plus polarization. } \\
\text { Like DND includes a polarization } \\
\text { p-function on all hydrogen atoms. } \\
\text { Best accuracy, highest cost. Impor- } \\
\text { tant for hydrogen bonding. }\end{array}$ & $\begin{array}{l}\text { H: 1s 1s' 1p } \\
\text { C: 1s 2s 2p 2s' 2p' 3d } \\
\text { Si: 1s 2s 2p 3s 3p 3s' 3p' } 3 d\end{array}$ \\
\hline TNP & $\begin{array}{l}\text { Triple Numerical plus polarization. } \\
\text { Like DNP including additional po- } \\
\text { larization functions on all atoms. } \\
\text { Available only for } \mathrm{H} \text { to } \mathrm{Cl} \text { except } \mathrm{He} \\
\text { and Ne. Best accuracy, highest cost. }\end{array}$ & 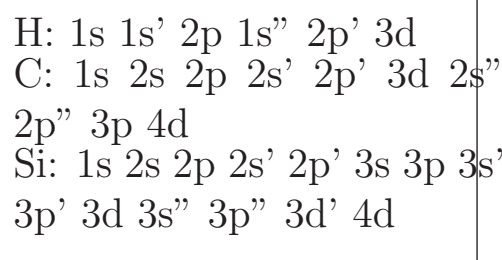 \\
\hline
\end{tabular}




\subsection{DFT calculation of periodic structures}

Cut-off energy. Within the Kohn-Sham framework, the non-interacting kinetic energy, $\hat{T}$, the Hartree energy, $V_{H}$, and other terms in Eq. (2.1) are more easily evaluated in reciprocal space rather than real space. For example, if we write a KSorbital as a sum of plane waves $\phi_{i \mathbf{k}}(r)=\sum_{\mathbf{G}} c_{i \mathbf{K}}(\mathbf{G}) \exp ((\mathbf{k}+\mathbf{G}) \cdot \mathbf{r})$ and apply the single-particle kinetic energy operator, we have: $T=\frac{1}{2} \sum_{i \mathbf{k}} \sum_{\mathbf{G}}(\mathbf{k}+\mathbf{G})^{2}$. The effect of the kinetic energy operator in reciprocal space is thus to multiply each plane wave coefficient by $\frac{1}{2}$ times the square of its wave vector. From similar consideration we can conclude that the Hartree potential reads: $V_{H}=\frac{1}{2} \sum_{\mathbf{G}} \rho^{*}(\mathbf{G}) \rho(\mathbf{G})$, where $\rho(\mathbf{G})$ is the corresponding Fourier component of electron density. The more components are involved into Fourier expansion, there more precise is the result. The cut-off energy defines a highest frequency Fourier component which may be present in $u(r)$. In turn, this defines a maximum required sampling density in real space. The cut-off $\mathbf{G}_{c u t}$ is usually expressed as an energy, where one is referring to the kinetic energy of a plane wave with this g-vector, so that $E_{c u t}=\frac{\mathbf{G}^{2}}{2}$. Practical convergence studies of graphene are presented in paragraph 5.1.2

Brillouin Zone (BZ) sampling. If $V_{\text {ext }}$ in Eq. (2.2) has translational symmetry, it does not follow that its solution $\psi(r)$ has the same translation symmetry. The Bloch's Theorem proves that if $V$ has translational symmetry, the solutions can be written $\psi_{\mathbf{k}}(\mathbf{r})=\exp (i \mathbf{k} \cdot \mathbf{r}) u_{\mathbf{k}}(\mathbf{r})$, where $u(\mathbf{r})$ has the same periodicity as $V$, and $\mathbf{k}$ lies 
within the Brillouin Zone (BZ), i.e.

$$
\begin{aligned}
& -\frac{1}{2}<k_{l} \leq \frac{1}{2} \\
& -\frac{1}{2}<k_{m} \leq \frac{1}{2} \\
& -\frac{1}{2}<k_{n} \leq \frac{1}{2},
\end{aligned}
$$

where

$$
\mathbf{k}=k_{l} \mathbf{a}^{*}+k_{m} \mathbf{b}^{*}+k_{n} \mathbf{c}^{* 2}
$$

This ensures that there is only one value of $\mathbf{k}$ for any given orbital, $\phi(\mathbf{r})$. The Bloch wave vector, $(\mathbf{k})$, of a given orbital could lie anywhere within the first Brillouin zone. For a general system there should in principle, be a set orbitals for every possible value of $\mathbf{k}$. Observables are calculated as an integral over all k-points within the 1st BZ. For example:

$$
\begin{aligned}
& E_{t o t}=\frac{1}{\Omega_{B Z}} \int_{B Z} E_{\mathbf{k}} d^{3} \mathbf{k} \\
& \rho(\mathbf{r})=\frac{1}{\Omega_{B Z}} \int_{B Z} \phi_{\mathbf{k}} \phi_{\mathbf{k}}^{*} d^{3} \mathbf{k}
\end{aligned}
$$

In practice, the integration is always replaced by a weighted sum over special k-points:

$$
\frac{1}{\Omega_{B Z}} \int_{B Z} \longrightarrow \sum_{\mathbf{k}} w_{\mathbf{k}}
$$

i.e. we only deal with a finite set of k-points, usually distributed evenly throughout the Brillouin zone according to a Monkhorst-Pack scheme [51]. Usually the shifted Monkhorst-Pack mesh is preferred over the conventional one. For hexagonal lattices,

\footnotetext{
${ }^{2} \mathbf{a}^{*}, \mathbf{b}^{*}, \mathbf{c}^{*}$ are the primitive vectors in the reciprocal space, defined through the primitive vectors in the direct space $(\mathbf{a}, \mathbf{b}, \mathbf{c})$ as: $\mathbf{a}^{*}=2 \pi \frac{\mathbf{b} \times \mathbf{c}}{\mathbf{a} \cdot(\mathbf{b} \times \mathbf{c})} \mathbf{b}^{*}=2 \pi \frac{\mathbf{a} \times \mathbf{c}}{\mathbf{a} \cdot(\mathbf{b} \times \mathbf{c})} \mathbf{c}^{*}=2 \pi \frac{\mathbf{a} \times \mathbf{b}}{\mathbf{a} \cdot(\mathbf{b} \times \mathbf{c})}$
} 
the shift vector is $\left(\begin{array}{lll}0.0 & 0.0 & 0.5\end{array}\right)$. If the system under consideration has nontrivial symmetry, it makes the k-points mutually connected by the symmetry operators to have the same eigenfunctions, making filling of the whole BZ with k-points redundant. In this case only a symmetry independent volume of BZ is used. This volume is called the Irreducible Brillouin Zone (IBZ).

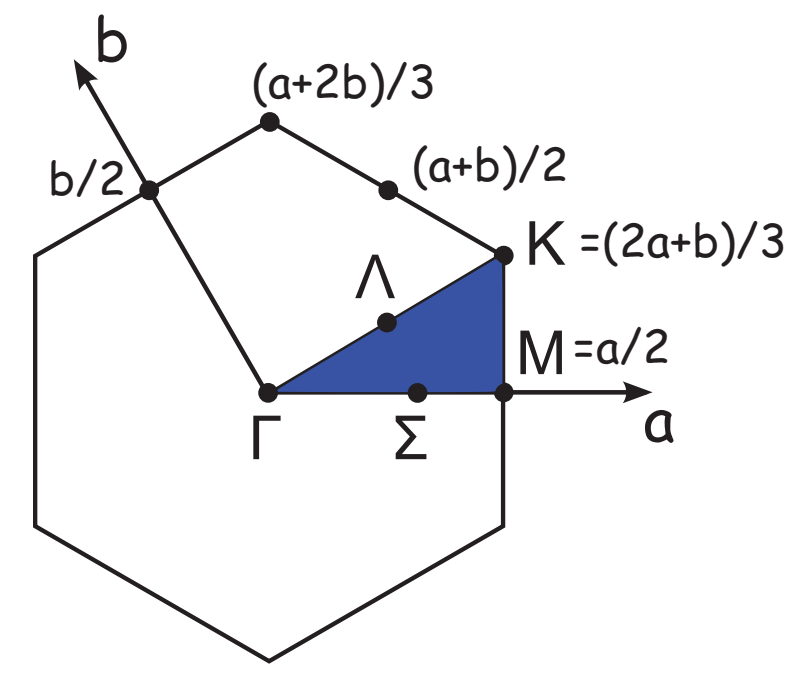

Figure 2.2: First Brillouin Zone of a graphene hexagonal unit cell and the IBZ part of it (blue)

Of course the denser is the Monkhorst-Pack mesh, the more complete is the expansion basis, the preciser are the observables calculated. Therefore it is important to perform convergence studies with respect of the number of k-points. Undersampling of the BZ (eg. using only one k-point $\Gamma=(0,0,0))$ maximizes the unwanted interaction between the periodic images, as all the images are in phase, and thus forming bonding orbitals.

For 2D systems, such as graphene, the compromise is between using a plane of k-points with $k_{z}=0$, and noting that inversion symmetry in k-space will halve the number of points needed, although the interaction between the slab and its periodic image will be maximized. Using $k_{z}=1 / 4$, where inversion symmetry just adds 
another $k_{z}=-1 / 4$ plane, so twice as many k-points will be needed, but the interaction between periodic images will be reduced so less vacuum may be needed.

However, k-points in IBZ fully represent the properties of the structure examined, however, for most of the post-processing calculations a k-point mesh in the full BZ is required. Also Wannierization and other post-processing algorithms work more effectively on the k-point grids that contain gamma point $[52,53]$.

\subsection{Supercells and Brillouin zone folding}

In order to control the degree of functionalization (see below in sections 6.4 and 7.2), we need to consider supercell of different sizes (i.e. one functionalizing molecule per $1 \times 1$ or $2 \times 2$ or $6 \times 6$ graphene supercell). As a result of consideration of a supercell, a so called Brillouin zone folding occurs.

As it was discussed in Sec. 2.5, the first BZ contains complete information about the possible values of the $k$ vector in the crystal. Therefore the Bloch waves in a crystal can be completely characterized by their behavior in a single Brillouin zone. However, numerical calculations of nanostructure electronic properties are often based on nonprimitive unit cells (supercells), usually constructed by multiplication of a primitive cell in some or all spatial dimensions. Sometimes a more complex transformation matrix (such as e.g. Eq. (4.6)) can be convenient for description of a hexagonal cell in the rectangular setting because the rectangular geometry allows for both highly efficient algorithms and ease of debugging. Those two cases, however, create a drawback called the Brillouin zone folding. This phenomenon arises from Eq. (2.10): if a unitcell is multiplied in the direct space, the $\mathbf{a}^{*}, \mathbf{b}^{*}, \mathbf{c}^{*}$ vectors become correspondingly shorter reducing the possible range for the $\mathbf{k}$ vectors.

Consider e.g. a $3 \times 1 \times 1$ supercell, i.e. a supercell made by three times repetition 


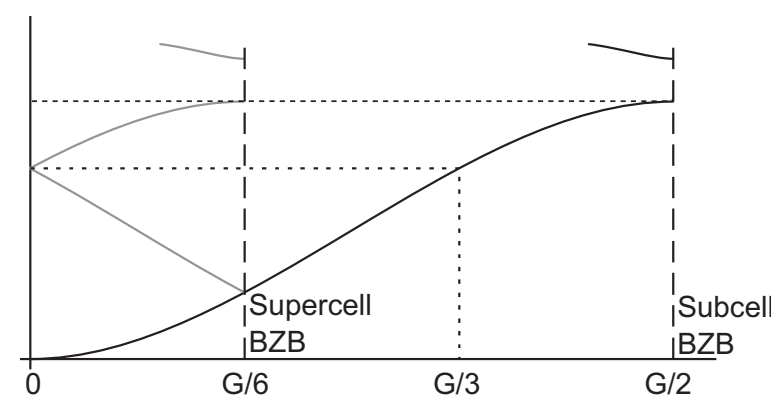

Figure 2.3: One dimensional BZ folding in a 3x1x1 super cell

of the subcell in the $x^{*}$ direction. The positions of the energy levels at $k=0$ in the supercell formed by three primitive cells should be identical to that at $k=0, \frac{1}{3}$, and $\frac{2}{3}$ in the primitive cell. Furthermore, $k=\frac{2}{3}$ should be equivalent to $k=-\frac{1}{3}$, and by inversion symmetry $k=-\frac{1}{3}$ is equivalent to $k=\frac{1}{3}$. Electron energy $E(\mathbf{k})$ is periodic, and obeys $E(\mathbf{k})=E(-\mathbf{k})$. Perturbation theory produces a discontinuity in $E(\mathbf{k})$ at points where $k$ corresponds to a periodicity in the potential, i.e. on the edges of the BZ. In the Fig. 2.3 a single band extends across the whole of the reciprocal space unit cell. The start of the next band is also shown. As $E(\mathbf{k})$ is an even function, negative values of $\mathrm{k}$ are omitted. In the $3 \times 1 \times 1$ supercell, the Brillouin Zone is a third of the length of the BZ of the subcell, and the k-points which now lie outside this Brillouin Zone can be translated into it by subtracting multiples of the new reciprocal lattice vectors. This process is conventionally called "folding"

The new supercell contains three times as many electrons as the old unit cell, so it needs to have three times as many bands to host them. The "folding" of the original BZ triples the number of bands in the supercell BZ, serving exactly this purpose. It is important to point put that at the new BZ boundaries there are no band-gaps, and the derivative of $E(\mathbf{k})$ is not zero. Only at those points which correspond to a periodicity in the potential does this behavior occur, providing a possibility to "unfold" the BZ. In the two dimensions, however, this process becomes much more complex because 
folding now affects translations in two dimensions. All k-vectors belonging th the larger subcell BZ should be mapped into the smaller supercell BZ, or equivalently, second, third, etc. BZ of the subcell are mapped into its first BZ. The folding process is straightforward - the coordinates of the k-points of the subcell BZ are transformed into the coordinates of the k-points of the supercell BZ by dividing them modulo $\mathbf{G}$, where $\mathbf{G}$ is the vector connecting the $\Gamma$ point (center of the BZ) with the edge of the supercell BZ in the direction of the k-vector. This process can be illustrated as following:
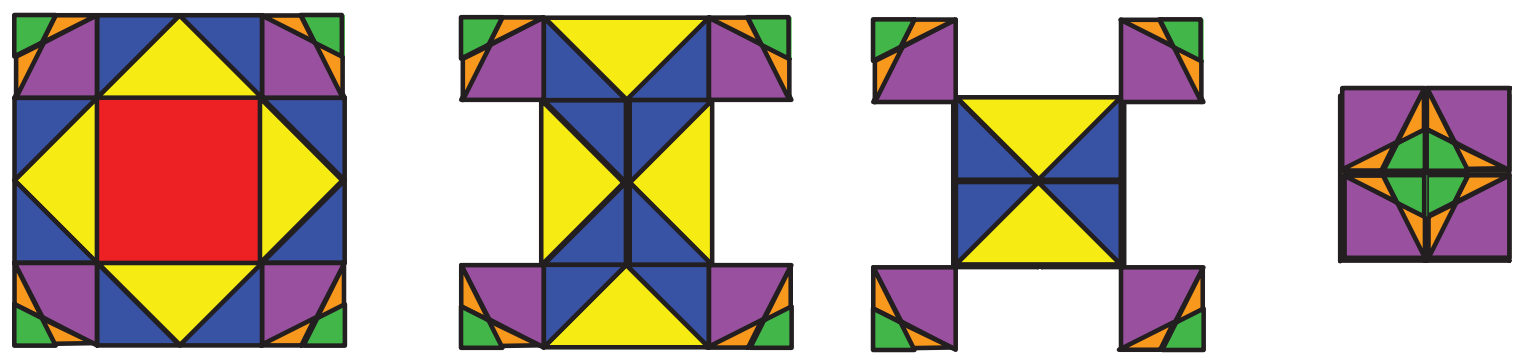

Figure 2.4: Mapping of the second BZ of a square lattice onto the first BZ

In Fig. 2.4 the left image represents the BZ of the subcell that is being consequently mapped into the red square representing the BZ of the $2 \times 2 \times 1$ supercell. From that folding algorithm we conclude that the $\Gamma-X$ path in the $2 \mathrm{x} 2 \mathrm{x} 1$ supercell BZ would consist of the following four sections in the subcell BZ:

1) $\Gamma(0,0,0) \longrightarrow\left(\frac{1}{4}, 0,0\right)$

2) $X\left(-\frac{1}{2}, 0,0\right) \longrightarrow\left(-\frac{1}{4}, 0,0\right)$

3) $M\left(-\frac{1}{2}, \frac{1}{2}, 0\right) \longrightarrow\left(-\frac{1}{4}, \frac{1}{2}, 0\right)$

4) $Y\left(0, \frac{1}{2}, 0\right) \longrightarrow\left(\frac{1}{4}, \frac{1}{2}, 0\right)$

Overlap of these four sections will give exactly the $\Gamma-X$ band structure of the $2 \times 2 \times 1$ supercell.

The process becomes much more complex for non-rectangular lattices, but it gains special importance because of graphene hexagonal structure. In Fig. 2.5 the left im- 

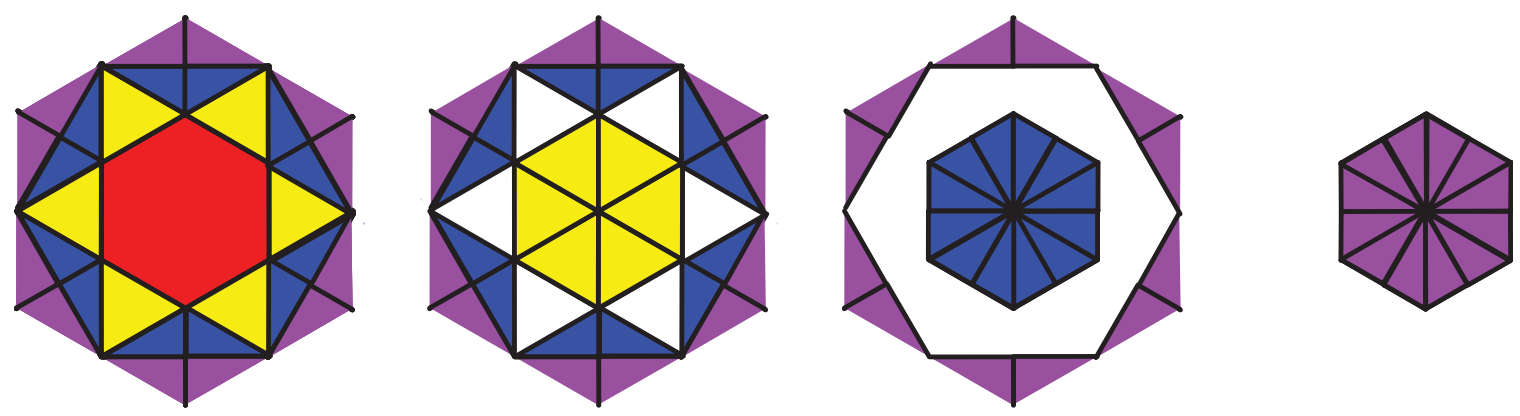

Figure 2.5: Mapping of the second BZ of a hexagonal lattice onto the first BZ

age represents the $\mathrm{BZ}$ of the subcell that is being consequently mapped into the red hexagon representing the $\mathrm{BZ}$ of the $2 \times 2 \times 1$ supercell. In this case exact mapping of each section of the subcell BZ onto the supercell BZ is too cumbersome, therefore we will just outline on several important BZ features: the $M$ point is mapped into the $\Gamma$, whereas the Dirac point $K$ remains invariant with respect to the folding procedure. This will become very important later because it is the Dirac point where the valence band and the conduction band touch each other making pristine graphene a metal. Thus modification of the electronic structure of graphene about this particular point is the main goal of the functionalization procedure. The studies of funtionalized graphene are conducted on supercells, whose size varies from $2 \times 2 \times 1$ to $6 \times 6 \times 1$ depending on the concentration of functionalizing molecules (one molecule per supercell).

Since most ab-initio programs can also handle supercells, it is natural to apply them to these structures as well. However, problems arise due to the fact that the rectangular unit cell is generally not the primitive cell of the superlattice, so that the resulting $E(\mathbf{k})$ relations must be unfolded to obtain the primitive-cell $E(\mathbf{k})$ curves. If all of the primitive cells in the rectangular unit cell are identical, then the unfolding is reasonably straightforward; if not, the problem becomes more difficult. 


\section{CHAPTER III}

\section{Quantum transport in dimensionally reduced \\ structures}


In this chapter I will discuss theoretical and numerical aspects of electron transport in spatially localized structures. A very good introduction to quantum transport can be found in [54]. This chapter follows the ideas discussed therein.

\subsection{Electronic transport in nanostructures}

The quantum transport theory was pioneered by Landauer and Büttiker $[55,56]$. Since than it has gained a widespread attention due to both the enhancement of experimental techniques and improvements in the theoretical methods, based on Keldysh formalism [11], and the non-equilibrium Green's functions (NEGF), in particular (See e.g $[57])$.

Here a method is discussed to compute transport though a nanodevice connected to two electrodes with chemical potentials (in this paragraph and in future I will use both Fermi level and (electro)chemical potential with the same meaning) $\mu_{L}$ (left) and $\mu_{R}$ (right) by employment of the NEGF formalism. If $\mu_{L}$ and $\mu_{R}$ are not equal due to external bias, i.e. $\mu_{L}-\mu_{R}=e \varphi$, the nanodevice is not in equilibrium, and there is a net current flow from left to right (See Fig. 3.1).

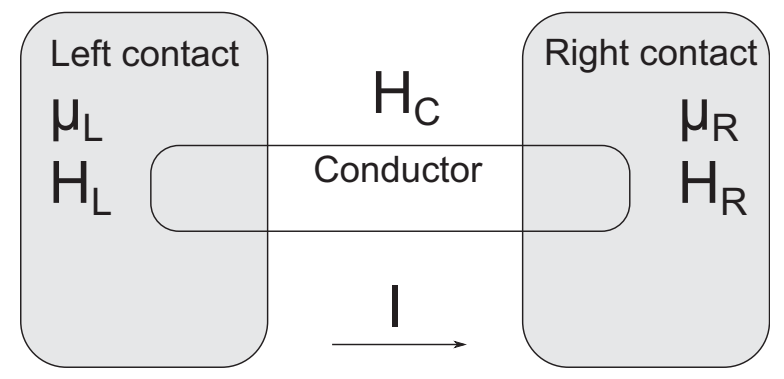

Figure 3.1: Nanodevice coupled to semi-infinite contacts with different Fermi levels $\mu_{L}$ (left) and $\mu_{R}$ (right). $\mathbf{H}_{\mathbf{C}}$ denotes the conductor $(\mathrm{C})$ Hamiltonian, $\mathbf{H}_{\mathbf{L}}$ and $\mathbf{H}_{\mathbf{R}}$ are the Hamiltonians of the left $(\mathrm{L})$ and the right $(\mathrm{R})$ electrodes

The electrodes are considered as semi-infinite electron reservoirs so that they are large enough that the bulk $\mu_{L}$ and $\mu_{R}$ are not affected by the current $I$. The leads 
can be described by one-particle Hamiltonians $H_{R}$ and $H_{L}$ respectively. The conventional way of treatment of such systems is to partition them into three regions: the nanodevice itself and two contacts and to perform calculation separately.

Neglecting the inelastic scattering in the system, the total Hamiltonian $\mathbf{H}$ can be partitioned as:

$$
H=\underbrace{H_{R}+H_{L}+H_{C}}_{H_{0}}+\underbrace{V_{L}+V_{R}}_{V}
$$

where $H_{0}$ is the Hamiltonian of three uncoupled regions, and $V$ is their coupling. From all eigenstates of $H$ we consider $\left|i_{+}\right\rangle$with energy $\varepsilon_{i}$ emitted from the left electrode, partially reflected back, and partially transmitted into the right electrode and the symmetric state $\left|j_{-}\right\rangle$with energy $\varepsilon_{j}$ emitted from the right lead. Since emitted (and absorbed back) elections do not change the properties of the electrodes, the states $\left|i_{+}\right\rangle$ $\left(\left|j_{-}\right\rangle\right)$are in equilibrium with the left (right) reservoir. Which means that the $\left|i_{+}\right\rangle$ $\left(\left|j_{-}\right\rangle\right)$states are occupied by electrons with the Fermi function $f\left(\varepsilon_{i}-\mu_{L}\right)\left(f\left(\varepsilon_{j}-\mu_{R}\right)\right)$. Consider the current circulating between the right electrode and the nanodevice:

$$
I=\left(\frac{d Q_{R}}{d t}\right)_{R}
$$

where $Q_{R}$ is the total charge in the right reservoir which is defined as:

$$
Q_{R}=-e \sum_{j \in R}\langle j|\rho| j\rangle
$$

where $\rho$ is the equilibrium density operator, and the states $|j\rangle$ are the eigenstates of $H_{R}, \frac{d \rho}{d t}$ is given by the Liouville equation:

$$
\frac{d \rho}{d t}=\frac{1}{i \hbar}\left[H_{R}, \rho\right]+\frac{1}{i \hbar}\left[V_{R}, \rho\right]
$$


Only the second term, associated with coupling should be considered because if $V_{R}=$ 0 , there is no current circulating though the interface.

The total current can be split into two contributions as $I=I^{+}+I^{-}$, where $I^{+}\left(I^{-}\right)$ comes from the electrons in state $\left|i_{+}\right\rangle\left(\left|j_{-}\right\rangle\right)$, injected from the left (right) electrode and scattered by potential $V_{R}\left(V_{L}\right)$. For $I^{+}$we have:

$$
I^{+}=\frac{-e}{i \hbar} \sum_{j \in R}\left\langle j\left|\left[V_{R}, \rho_{+}\right]\right| j\right\rangle,
$$

where $\rho_{+}$is the contribution into $\rho$ from the states $\left|i_{+}\right\rangle$:

$$
\rho_{+}=\sum_{j \in L}\left|i^{+}\right\rangle f\left(\varepsilon_{i}-\mu_{L}\right)\langle j|
$$

Substituting the later equation into the previous one, we obtain

$$
I^{+}=\frac{-e}{i \hbar} \sum_{j \in R} \sum_{j \in L}\left(\left\langle j\left|V_{R}\right| i_{+}\right\rangle\left\langle i_{+} \mid j\right\rangle-\left\langle i_{+} \mid j\right\rangle\left\langle i_{+}\left|V_{R}\right| j\right\rangle\right) f\left(\varepsilon_{i}-\mu_{L}\right) .
$$

The eigenstates $\left|i_{+}\right\rangle$are the solutions of the Schrödinger equation:

$$
\begin{aligned}
& \left(\varepsilon_{i}-H\right)\left|i_{+}\right\rangle=0 \\
& \left(\varepsilon_{i}-H_{0}\right)\left|i_{+}\right\rangle=V\left|i_{+}\right\rangle
\end{aligned}
$$

If $V=0$ (i.e. no coupling situation), the solution corresponds to the stationary equilibrium function, i.e. $\left|i_{+}\right\rangle \longrightarrow|i\rangle$, that corresponds to the eigenstates of $H_{0}$ :

$$
\left(\varepsilon_{i}-H_{0}\right)|i\rangle=0
$$


thus the formal solution of Eq. (3.5) is:

$$
\left|i_{+}\right\rangle=|i\rangle+\left(\varepsilon_{i}-H_{0}\right)^{-1} V\left|i_{+}\right\rangle
$$

To proceed further on we employ the Green's function formalism (see e.g. [34, 58]).

$$
\begin{aligned}
G_{0}(\varepsilon) & =\lim _{\eta \rightarrow 0^{+}}\left(\varepsilon-H_{0}+i \eta\right)^{-1} \\
G(\varepsilon) & =\lim _{\eta \rightarrow 0^{+}}(\varepsilon-H+i \eta)^{-1}
\end{aligned}
$$

The small factor $\eta$ is introduced for numerical convergence purposes. The Green's functions are connected by the Dyson's equation. Using the definition of the Green's function, Eq. (3.6) can be rewritten as:

$$
\left|i_{+}\right\rangle=|i\rangle+G_{0}\left(\varepsilon_{i}\right) V\left|i_{+}\right\rangle
$$

And vice versa, by reversing $V$ and changing $G_{0}$ to $G$ :

$$
\begin{aligned}
& |i\rangle=\left|i_{+}\right\rangle-G\left(\varepsilon_{i}\right) V|i\rangle \\
& \left|i_{+}\right\rangle=|i\rangle+G\left(\varepsilon_{i}\right) V|i\rangle
\end{aligned}
$$

Now, inserting $\left|i_{+}\right\rangle$from Eq. (3.8) into Eq. (3.4) and using the fact that since $|i\rangle$ and $|j\rangle$ belong to different electrodes, $\langle i \mid j\rangle=0$ we obtain:

$$
I^{+}=\frac{-e}{i \hbar} \sum_{j \in R} \sum_{j \in L}\left(\langle j|V+V G V| i\rangle\left\langle i\left|V G^{\dagger}\right| j\right\rangle-\langle j|G V| i\rangle\left\langle i\left|V+V G^{\dagger} V\right| j\right\rangle\right) f\left(\varepsilon_{i}-\mu_{L}\right) .
$$


In the Eq. (3.9) we replace $G$ and $G^{\dagger}$, using the Dyson's equation,

$$
\begin{aligned}
& G=G_{0}+G_{0} V G \\
& G^{\dagger}=G_{0}^{\dagger}+G^{\dagger} V G_{0}^{\dagger}
\end{aligned}
$$

Then:

$$
\begin{aligned}
\langle j|G V| i\rangle & =\left\langle j\left|G_{0} V+G_{0} V G V\right| i\right\rangle=\left\langle j\left|G_{0} V\right| i\right\rangle+\left\langle j\left|G_{0} V G V\right| i\right\rangle \\
& =\frac{\langle j|V| i\rangle}{\varepsilon_{i}-\varepsilon_{j}+i \eta}+\frac{\langle j|V G V| i\rangle}{\varepsilon_{i}-\varepsilon_{j}+i \eta}=\frac{\langle j|V+V G V| i\rangle}{\varepsilon_{i}-\varepsilon_{j}+i \eta} \\
\left\langle i\left|V G^{\dagger}\right| j\right\rangle & =\left\langle i\left|V G_{0}^{\dagger}+V G^{\dagger} V G_{0}^{\dagger}\right| j\right\rangle=\left\langle i\left|V G_{0}^{\dagger}\right| j\right\rangle+\left\langle i\left|V G^{\dagger} V G_{0}^{\dagger}\right| j\right\rangle \\
& =\frac{\langle i|V| j\rangle}{\varepsilon_{i}-\varepsilon_{j}-i \eta}+\frac{\left\langle i\left|V G^{\dagger} V\right| j\right\rangle}{\varepsilon_{i}-\varepsilon_{j}-i \eta}=\frac{\left\langle i\left|V+V G^{\dagger} V\right| j\right\rangle}{\varepsilon_{i}-\varepsilon_{j}-i \eta},
\end{aligned}
$$

Thus Eq. (3.9) simplifies further as:

$$
I^{+}=\frac{-e}{i \hbar} \sum_{j \in R} \sum_{j \in L}\left(\frac{\langle j|t| i\rangle\left\langle i\left|t^{\dagger}\right| j\right\rangle}{\varepsilon_{i}-\varepsilon_{j}-i \eta}-\frac{\langle j|t| i\rangle\left\langle i\left|t^{\dagger}\right| j\right\rangle}{\varepsilon_{i}-\varepsilon_{j}+i \eta}\right) f\left(\varepsilon_{i}-\mu_{L}\right)
$$

Where the notation $t(\varepsilon)=V+V G(\varepsilon) V$ was used. In the limit $\eta \rightarrow 0^{+}$Eq. (3.10) becomes:

$$
I^{+}=2 \pi \frac{-e}{\hbar} \sum_{j \in R} \sum_{j \in L}|\langle j|t| i\rangle|^{2} f\left(\varepsilon_{i}-\mu_{L}\right) \delta\left(\varepsilon_{i}-\varepsilon_{j}\right)
$$

Using the similar equation for $I^{-}$the total current becomes:

$$
I=\frac{2 \pi e}{\hbar} \sum_{j \in R} \sum_{j \in L}|\langle j|t| i\rangle|^{2}\left(f\left(\varepsilon_{j}-\mu_{R}\right)-f\left(\varepsilon_{i}-\mu_{L}\right)\right) \delta\left(\varepsilon_{i}-\varepsilon_{j}\right) .
$$

The matrix element of the scattering operator $t$ is expresses as:

$$
t_{i j}=\langle i|t| j\rangle=\langle i|V| j\rangle+\langle i|V G V| j\rangle=\langle i|V| n\rangle\langle n|G| m\rangle\langle m|V| j\rangle=\sum_{n, m} V_{i n} G_{n m} V_{m j}
$$


where $|m\rangle$ and $|n\rangle$ denote eigenstates of $H_{C}$. Since two leads are only coupled through the nanodevice, $\langle i|V| j\rangle=0$. Using the hermiticity of the Green's function, $\left|t_{i j}\right|^{2}=$ $\sum_{n, m, n^{\prime}, m^{\prime}} V_{i n} G_{n m} V_{m j} V_{n^{\prime} i} G_{m^{\prime} n^{\prime}}^{\dagger} V_{j m^{\prime}}$. Defining two coupling matrices (weighted density of states ${ }^{1}$ in the left and right electrodes respectively)

$$
\begin{aligned}
& \Gamma_{n m}^{L}=2 \pi \sum_{i \in L} V_{n i} V_{i m} \delta\left(\varepsilon-\varepsilon_{i}\right) \\
& \Gamma_{n m}^{R}=2 \pi \sum_{j \in R} V_{n j} V_{j m} \delta\left(\varepsilon-\varepsilon_{j}\right),
\end{aligned}
$$

the scattering operator matrix element becomes:

$$
\left|t_{i j}\right|^{2}=\sum_{n, m, n^{\prime}, m^{\prime}} \Gamma_{n n^{\prime}}^{L}(\varepsilon) \Gamma_{m m^{\prime}}^{R}(\varepsilon) G_{n m}(\varepsilon) G_{m^{\prime} n^{\prime}}^{\dagger}(\varepsilon)=\operatorname{Tr}\left[\Gamma^{L} G \Gamma^{R} G^{\dagger}\right]
$$

where the trace is taken over all states in the decoupled nanodevice only. Now Eq. (3.12) can be rewritten $\mathrm{as}^{2}$ :

$$
I=\frac{e}{h} \int \mathcal{T}(\varepsilon)\left(f\left(\varepsilon-\mu_{R}\right)-f\left(\varepsilon-\mu_{L}\right)\right) d \varepsilon,
$$

where

$$
\mathcal{T}(\varepsilon)=\operatorname{Tr}\left[\Gamma^{L} G \Gamma^{R} G^{\dagger}\right]
$$

is the transmission function through the nanodevice, $\mathcal{T}$ should be multiplied by a factor 2 to account for spin degeneracy ${ }^{3}$. The famous Landauer formula [55] can be

\footnotetext{
$\left.\varepsilon_{i}\right)$

${ }^{1}$ Normal densities of states are defined as $D o S_{R}(\varepsilon)=\sum_{j \in R} \delta\left(\varepsilon-\varepsilon_{j}\right)$ and $D o S_{L}(\varepsilon)=\sum_{j \in L} \delta(\varepsilon-$

${ }^{2}$ The summation over states in the electrodes is replaced by integration due to the introduction of the density of states, i.e. $\sum_{j \in R} \sum_{j \in L} \delta\left(\varepsilon_{i}-\varepsilon_{j}\right)=\int \sum_{j} \delta\left(\varepsilon-\varepsilon_{j}\right) \sum_{j} \delta\left(\varepsilon-\varepsilon_{i}\right) d \varepsilon$

${ }^{3}$ Despite the fact that we derived this expression for electrons, the same approach can be utilized to derive the heat transport equation through a nanodevice (i.e. consider phonons instead of electrons). In this case $f$ will denote the Bose-Einstein distribution instead of the Fermi-Dirac. Of course there is no factor 2 in that case.
} 
derived from Eq. (3.14) under assumption of infinitely small bias $(d V)$ :

$$
\begin{aligned}
I & =\frac{2 e}{h} \int \mathcal{T}(\varepsilon)\left(f\left(\varepsilon-\varepsilon_{F}-e d V\right)-f\left(\varepsilon-\varepsilon_{F}\right)\right) d \varepsilon \\
G & =\frac{d I}{d V}=\frac{2 e^{2}}{h} \int \mathcal{T}(\varepsilon) \underbrace{\left(-\frac{d f}{d V}\right)}_{\approx \delta\left(\varepsilon-\varepsilon_{F}\right)} d \varepsilon \\
G & =\frac{2 e^{2}}{h} \mathcal{T}\left(\varepsilon_{F}\right)=G_{0} \mathcal{T}\left(\varepsilon_{F}\right),
\end{aligned}
$$

where $G_{0}=\frac{2 e^{2}}{h}$ is the quantum of conductance.

Conductance versus conductivity. Only conductance is quantized in multiples of $G_{0}$, however, a measurable property of the material is not conductance but conductivity $(\sigma)$. Conductivity id related to conductance by $G=\sigma \frac{A}{\ell}$, where $\ell$ is the length of the conductor, measured in meters [m], $A$ is the cross-section area of the conductor measured in square meters $\left[\mathrm{m}^{2}\right], \sigma$ is the electrical conductivity measured in siemens per meter $\left(\mathrm{Sm} \cdot \mathrm{m}^{-1}\right)$. If a $2 \mathrm{D}$ material is considered, $A$ becomes the length of the conductor in the lateral direction and the units of conductivity and conductance are same.

This consideration becomes especially important when GNRs or different lattice settings are considered, e.g. conductance of graphene in the $\Gamma-K$ direction will be $\sqrt{3}$ times higher in the orthorhombic lattice than in the hexagonal due to the fact that the $b$-lattice parameter, obtained from the transformation Q (see Eq (4.6)) is $\sqrt{3}$ times longer than in the hexagonal setting. In case of GNRs due to BZ folding, the number of bands in the first BZ increases, also increasing the ballistic conductance. However, since the number of bands is proportional to the number of atoms, which in turn, is proportional to the width of the GNR, conductivity virtually remains same. Self evidently, all this is true for thermal conductivity an conductance as well. 


\subsection{Quantum transport in the localized representation}

From the definition of the Green's function Eq. (3.7) it follows:

$$
\mathbf{G}=\left(\begin{array}{ccc}
(\varepsilon+i \eta) \mathbf{I}-\mathbf{H}_{\mathbf{L}} & -\mathbf{V}_{\mathbf{L}} & 0 \\
-\mathbf{V}_{\mathbf{L}}^{\dagger} & (\varepsilon+i \eta) \mathbf{I}-\mathbf{H}_{\mathbf{C}} & -\mathbf{V}_{\mathbf{R}} \\
0 & -\mathbf{V}_{\mathbf{R}}^{\dagger} & (\varepsilon+i \eta) \mathbf{I}-\mathbf{H}_{\mathbf{R}}
\end{array}\right)^{-1}
$$

where $\mathbf{H}_{\mathbf{R}}, \mathbf{H}_{\mathbf{L}}$, and $\mathbf{H}_{\mathbf{C}}$ are the Hamiltonian matrices (See Eq. (2.9) and (3.1)) in the $\mathrm{L}, \mathrm{R}$ and $\mathrm{C}$ regions, respectively, and $\mathbf{V}_{\mathbf{L}}\left(\mathbf{V}_{\mathbf{R}}\right)$ is the coupling matrix between the $\mathrm{L}(\mathrm{R})$, and $\mathrm{C}$ regions, and $\mathbf{I}$ is the unit matrix. Only the Green's function the nanodevice of $\mathbf{G}_{\mathbf{C}}$ is affecting its transport properties.

$$
G_{C}=G_{22}=\left((\varepsilon+i \eta) \mathbf{I}-\mathbf{H}_{\mathbf{C}}-\boldsymbol{\Sigma}\right)^{-1},
$$

where

$$
\begin{gathered}
\Sigma=V_{R} G_{0 R} V_{R}^{\dagger}+V_{L} G_{0 L} V_{L}^{\dagger}=\Sigma_{R}+\Sigma_{L} \\
G_{0 L}=G_{11}=\left((\varepsilon+i \eta) \mathbf{I}-\mathbf{H}_{\mathbf{L}}\right)^{-1} \\
G_{0 R}=G_{33}=\left((\varepsilon+i \eta) \mathbf{I}-\mathbf{H}_{\mathbf{R}}\right)^{-1}
\end{gathered}
$$

The coupling of $\mathrm{L}$ and $\mathrm{R}$ to the remaining part of the semi-infinite electrodes (replaced by a periodic structure) is fully taken into account by the self-energies, $\Sigma_{\mathbf{L}}$ and $\Sigma_{\mathbf{R}}$, which replace the discreet spectrum of nanodevice by a continuous one. Replacement of the semi-infinite electrode by a periodically repeated supercell is accounted by introducing self-energies that fully incorporate the perturbation caused by this simplification. In this case the Hamiltonian matrix can be rewritten in order 
to incorporate the effects of self energy:

$$
\mathbf{H}=\left(\begin{array}{ccc}
\mathbf{H}_{\mathbf{L}}+\boldsymbol{\Sigma}_{\mathbf{L}} & \mathbf{V}_{\mathbf{L}} & 0 \\
\mathbf{V}_{\mathbf{L}}^{\dagger} & \mathbf{H}_{\mathbf{C}} & \mathbf{V}_{\mathbf{R}} \\
0 & \mathbf{V}_{\mathbf{R}}^{\dagger} & \mathbf{H}_{\mathbf{R}}+\boldsymbol{\Sigma}_{\mathbf{R}}
\end{array}\right)
$$

The self energy is a complex non-hermitian, energy dependent operator. Its imaginary part is related to the coupling matrices by:

$$
\begin{aligned}
& \Gamma_{n m}^{L}=-2 \Im\left(\Sigma_{n m}^{L}\right) \\
& \Gamma_{n m}^{R}=-2 \Im\left(\Sigma_{n m}^{R}\right)
\end{aligned}
$$

However, the expression for $\Sigma$ greatly simplifies if the matrices $G_{0 R}$ and $G_{0 L}$ are written in the basis of eigenstates of the free electrodes $|i\rangle$ and $|j\rangle$ for the right and left electrodes respectively. The main advantage of the eigenbasis is that these matrices appear diagonal in this representation. The matrix elements of $\Sigma$ are given by:

$$
\Sigma_{n m}=\Sigma_{n m}^{R}+\Sigma_{n m}^{L}=\sum_{i \in L} \frac{V_{n i} V_{i m}}{\varepsilon-\varepsilon_{i}+i \eta}+\sum_{j \in R} \frac{V_{n j} V_{j m}}{\varepsilon-\varepsilon_{j}+i \eta},
$$

where the indices $n, m$ refer to the nanodevice.

Evaluation of the contacts' Green's functions require inversion of the infinite matrices. However, if one writes them in the real space or tight binding approximation, only the the matrix element at the surface of the leads and in the vicinity of the nanodevice are needed because the coupling $V$ describes short range interaction. This approach is far more computationally beneficial that the traditional reciprocal space representation of the Hamiltonian and the Bloch waves approach to its solutions. Bloch orbitals, that are intrinsically delocalized, have to be transformed into local- 
ized functions in order to construct the sparse, short-ranged matrix elements of the Hamiltonian. A well established way of doing so is using the (maximally-localized) Wannier functions (ML)WF for the system considered. Construction of these functions will we outlined in Sec. 3.3. If a localized representation of the total Hamiltonian of the system is chosen, the later can be written in form of a finite sparse matrix [59]. Than recursion or decimation methods [54] provide a robust algorithm to calculate self energies.

\subsection{Wannier functions and disentangled bands}

The (Maximally localized) Wannier functions ((ML)WF) are the most natural choice for a set of localized orbitals that still span the same Hilbert space of the Hamiltonian eigenfunctions: they allow to bridge Bloch-wave electronic structure and lattice Green's function calculations in a coherent fashion. Wannier functions are defined as [60]:

$$
w(\mathbf{R}, \mathbf{r})=\frac{1}{\sqrt{N}} \sum_{\mathbf{k}} \psi_{\mathbf{k}}(\mathbf{r}) e^{-i \mathbf{k} \cdot \mathbf{R}}=\frac{1}{\Omega_{B Z}} \int_{B Z} \psi_{\mathbf{k}}(\mathbf{r}) e^{-i \mathbf{k} \cdot \mathbf{R}} d^{3} k .
$$

Here $R_{n}$ denote Bravais vectors and $\Omega_{B Z}$ is the volume of the Brillouin zone (BZ). This definition, however is not unique and suffers from the indeterminacy of the phases of Bloch functions [61], $\psi_{\mathbf{k}}(\mathbf{r}) \rightarrow \psi_{\mathbf{k}}(\mathbf{r}) e^{\phi(\mathbf{k})}$. Furthermore, if a group of bands, m, is considered, with corresponding Bloch functions, $\psi_{m, \mathbf{k}}(\mathbf{r})$, due to the entanglement of the bands, the above definition should be generalized to:

$$
|\mathbf{R}, n\rangle=w_{n}(\mathbf{R}, \mathbf{r})=\frac{1}{\Omega_{B Z}} \int_{B Z}\left[\sum_{m=1}^{N} U_{m n}(\mathbf{k}) \psi_{m, \mathbf{k}}(\mathbf{r})\right] e^{-i \mathbf{k} \cdot \mathbf{R}} d^{3} k .
$$


where $U_{m n}(\mathbf{k})$ is an arbitrary unitary matrix. The unitarity is important to maintain the Wannier functions orthogonal. The arbitrariness of $U_{m n}(\mathbf{k})$ allows the tuning of the phases of the Bloch functions in the integral as well as the admixture of functions pertaining to different bands. The freedom to choose of the $U_{m n}(\mathbf{k})$ allows us to choose the most beneficial form of it: (Maximally localized) Wannier functions ((ML)WF), in which the Wannier function $w_{\mathbf{R}, \mathbf{r}}$ is localized around the point $\mathbf{R}$ and rapidly goes to zero away from $\mathbf{R}$. This method was pioneered by Marzari and Vanderbilt in 1997 [62]. Their concept of the minimum-spread criterion comes from the idea of extension of the molecular orbital concept into solid-state case, proposed by Boys [63]. If we consider the extreme case of isolated atoms, the Wannier function would become an isolated atomic orbital. That limit suggests the choice of an atomic wave function as an approximate form for the Wannier function, the so-called tight binding approximation.

\subsubsection{Localization procedure}

A measure of the spatial delocalization of WFs is given by a Spread Operator $\Omega$, defined as the sum of the second moments of all the Wannier functions in a reference $\operatorname{cell}[64]$ :

$$
\Omega=\sum_{n}\left[\left\langle r^{2}\right\rangle_{n}-\langle\mathbf{r}\rangle_{n}^{2}\right],
$$

where the sum is over a selected group of bands, and $\langle\mathbf{r}\rangle_{n}=\langle\mathbf{0} n|\mathbf{r}| \mathbf{0} n\rangle,\left\langle r^{2}\right\rangle_{n}=$ $\left\langle\mathbf{0} n\left|r^{2}\right| \mathbf{0} n\right\rangle$. The value of the spread $\Omega$ depends on the choice of unitary matrices $U^{(\mathbf{k})} ;$ thus it is possible to iterate any arbitrary set of $U^{(\mathbf{k})}$ until we reach the stationarity condition:

$$
\frac{\delta \Omega_{\mathbf{k}}}{\delta U^{(\mathbf{k})}}=0
$$


At minimum, we obtain the matrices $U_{M L}^{(\mathbf{k})}$, that transform the Bloch-function $\psi_{n \mathbf{k}}(\mathbf{r})$ into a Wannier-function $w_{n \mathbf{R}}(\mathbf{r})$ using Eq. (3.21). For the purpose of numerical convergence, the spread operator is usually decomposed into the gauge-invariant part $\left(\Omega_{I}\right)$, which is independent from the choice of $U_{M L}^{(\mathbf{k})}$, and two delocalized parts: $\Omega_{D}=\sum_{n} \sum_{R m \neq 0 m}|\langle\mathbf{R} n|\mathbf{R}| \mathbf{0} n\rangle|^{2}$ and $\Omega_{O D}=\sum_{m \neq n} \sum_{\mathbf{R}}|\langle\mathbf{R} m|\mathbf{R}| \mathbf{0} n\rangle|^{2}$. At the minimum, the elements $\langle\mathbf{R} m|\mathbf{R}| \mathbf{0} n\rangle$ are as small as possible.

Wannier functions obtained with the above procedure should be almost real, except for a phase factor that can be omitted by normalization of all functions. This property can also be used to check fpr the convergence of the localization procedure. Due to the crystal periodicity, even the above-mentioned WFs are not truly localized, but are periodic in real-space, with a superperiodicity determined by the BZ discretization. The truly isolated limit is recovered only in the case of continuous BZ integrations [65].

\subsection{Real space Hamiltonian representation and Interpolated band structure}

In order to calculate the conductance from the equations outlined in Sec. 3.2, we need matrix elements of the Hamiltonian to be calculated in the localized basis representation. Since Wannier functions contain exactly the same amount of information about quantum states of the system as Bloch functions, we can use the minimal set of MLWFs as a localized basis set. Assuming that BZ is sampled fine enough to eliminate the interaction with the WF from the neighboring cell, the Hamiltonian in the WF representation can simply be computed as $H_{i j}(\mathbf{R})=\langle\mathbf{0} i|H| \mathbf{R} j\rangle$. Using the unitary rotations $U(\mathbf{k})$ obtained in the localization procedure and the definition of the Wannier functions, we obtain the following: In the Bloch representation we 
have by definition $H_{m n}(\mathbf{k})=\varepsilon_{m \mathbf{k}} \delta_{m, n}$. Moving to the Wannier basis, first we have to "disentangle" the Hamiltonian matrix:

$$
H^{(r o t)}(\mathbf{k})=U(\mathbf{k})^{\dagger} H(\mathbf{k}) U(\mathbf{k}) .
$$

And then we do the Fourier transform of $H^{(r o t)}(\mathbf{k})$ into the corresponding set of Bravais lattice vectors $\mathbf{R}$ (see Eq. (3.21))

$$
H_{i j}(\mathbf{R})=\frac{1}{N_{\mathbf{k}}} \sum_{\mathbf{k}} H^{(r o t)}(\mathbf{k}) e^{-i \mathbf{k} \cdot \mathbf{R}}
$$

From disentangled Wannier functions and interpolated band structure can be obtained. Using the reciprocity relations between the Wannier and Bloch functions, orthonormal Bloch orbitals for any arbitrary state can be obtained as a Fourier series of the Wannnier functions:

$$
\psi_{i}^{\mathbf{k}}(r)=\frac{1}{\sqrt{N_{R}}} \sum_{\mathbf{R}} e^{i \mathbf{k} \cdot \mathbf{R}} \omega_{i}(r-\mathbf{R}) .
$$

Because of the nature of summation, $\psi_{i}^{\mathbf{k}}(r)$ now appears as a continuous function of k. This formulation is free from the ambiguity in the eigenvalues ordering created by diagonalization of the Hamiltonian matrix in the Bloch representation. This allows us to compute a continuous band structure, that properly handles band intersections. This type of band structure is called the disentangles band structure, for it allows the bands to actually intersect or not depending on their intrinsic symmetry. Unlike the case when a discreet set of k-points is chosen and bands are just calculated as ordered eigenvalues for each k-point, and thus the bands never intersect. 


\subsection{Practical transport calculations using the Green's func- tions}

The calculations of the conductivity with the NEGF approach were done using the Quantum-Espresso[66] and the WanT (Wannier-Transport) [52, 65] program suits. The typical sequence of the calculations is as follows:

1. First the self-consistent calculations are performed using Quantum-Espresso. These SCF calculations result in the self-consistent electron density as follows from Eq. (2.7).

2. After the SCF electron density is obtained, the $V_{H}$ and $V_{x c}$ functionals are computed, yielding closed form Kohn-Sham Hamiltonian $\left(H_{K S}\right)$ from Eq. (2.6). Using this Hamiltonian a nonSCF calculation is performed using the full set of k-points in BZ. On this step Bloch states wavefunctions are calculated for further processing. It is followed by a minor intermediate step - generation of the uniform k-point grid on which the required Bloch functions overlaps will be computed.

3. On the third step - the results of Quantum-Espresso calculations are passed to the diesntangle.x program, which is a part of the WanT program suite by means of a homemade interface script.

4. The code reads the energy window. From which it will extract a selected number (N) of WFs for further localization. For each k-point, the energy window must contain a number of bands not lower than N. Trial Wannier centers are not mandatory requirement in this step, however for better convergence they can be provided by the user. A careful choice of the trial WF it is recommended: usually WFs are chosen to be centered in the midpoints of the bonds. The 
number of WFs per bond can be deduced by examining which atomic orbitals contribute to the bond formation.

disentangle.x produces an output that keeps trace of the computed overlap and projection integrals (to be re-used in further or restarted calculations) and describes the Wannier optimal subspace.

5. After that wannier. $\mathrm{x}$ performs the localization and disentanglement procedures for the requested set of Wannier functions (See Sec. 3.3). The optimal unitary matrices $U(\mathbf{k})$ governing the transformation between Bloch and Wannier states are obtained. wannier. $\mathrm{x}$ writes two internal data files, containing the $\mathrm{U}(\mathrm{k})$ and a file with the Hamiltonian matrix in the localized representation (Eq. (3.22)), the inverse of which gives the Green's function of the system.

WanT has a number of postprocessing tools that are not directly required for the transport computation, but allow to keep track of the results of the disentanglement and localization procedures, and thus control the quality of calculated Hamiltonian matrix elements.

- bands.x: the code computes the interpolated band-structure of the system along a selected direction in the Brillouin Zone. The comparison with independently calculated DFT eigenvalues is a nice test to check the localization of the obtained WFs. When they are well behaved (i.e. localized), only few $\mathbf{R}$ lattice vectors are required to described the Hamiltonian on the Wannier basis $\left[H_{i j}(\mathbf{R})\right]$ Starting with a small set of $\mathbf{k}$ in the DFT calculation we obtain the Hamiltonian on the related (small) set of lattice vectors. When WFs are well localized the Hamiltonian is fully described on this set of $\mathbf{R}$ and we can diagonalize it for an arbitrary large set of $\mathbf{k}$ points (as a post-processing of the Wannier code). 
This is the interpolation of the band structure using WFs. If they are not localized we are essentially throwing away some non-negligible matrix elements in the Hamiltonian representation, and the bands are not accurate. Typical unphysical oscillations appear in these cases.

- dos.x: the code computes the density of states (DOS) of the system interpolating the band structure by means of WFs, as for bands.x. Since the DOS is a quantity integrated (or better summed) over the BZ, a uniform mesh of k points (supplied by the user) is adopted. Once a self-consistent calculation is performed (and WFs computed), this code can be used to obtain accurate DOS.

- plot. $\mathrm{x}$ : this is an utility to plot WFs in real space. The plotting region can be tuned and a generic number of WFs can be handled in a single run. The real or imaginary parts of the WFs as well as their squared moduli are allowed fields to be plotted. The code produces plot files in various formats (txt, gaussian cube, xsf, plt) allowing the use of standard open source visualization-packages.

Using the Hamiltonian matrices calculated in step 5 we can calculate both the bulk and the two-terminal transmittance. Except for the special case of the bulk transmittance (see below), the systems under consideration are not periodic along the transport direction, i.e. have defects, like adatoms, vacancies, and functionalizing molecules. This, however, does not exclude a 2D periodicity in the orthogonal plane, so transport across interfaces can be treated as well.

Using the Hamiltonian matrices, computed at step 5, we define following matrices, required for transport calculation. If leads are made of the same material, which is usually the case we are interested in, there is no difference between $H^{L C}$ and $H^{C R}$, $H_{00}^{L}$ and $H_{00}^{R}, H_{01}^{L}$ and $H_{01}^{R}$. The conductor calculation should contain part of the 
Table 3.1: Parameters of the Hamiltonians used for Wannier functions calculations

\begin{tabular}{llll}
\hline Symbol & Name & Formula & Value \\
\hline$N_{R}, N_{C}, N_{L}$ & number of Wannier functions & & $\begin{array}{l}\text { number of atomic orbitals per } \\
\text { bond x number of bonds }\end{array}$ \\
\hline$H_{00}^{L(R)}$ & $\begin{array}{l}\text { on-site hamiltonian of the left } \\
\text { (right) leads } \\
\text { hopping hamiltonian of the left } \\
\text { (right) leads }\end{array}$ & $N_{L(R)} \times N_{L(R)} \times N_{L(R)}$ & $\begin{array}{l}\text { Obtained from bulk calcula- } \\
\text { tion for left (right) lead } \\
\text { Obtained from bulk calcula- } \\
\text { tion for left (right) lead }\end{array}$ \\
\hline$H_{01}^{L(R)}$ & $\begin{array}{l}\text { on site hamiltonian of the con- } \\
\text { ductor C }\end{array}$ & $N_{C} \times N_{C}$ & $\begin{array}{l}\text { Obtained from bulk calcula- } \\
\text { tion for the conductor super- } \\
\text { cell } \\
H_{00}^{C}\end{array}$ \\
$\begin{array}{l}\text { coupling between lead L and con- } \\
\text { ductor C }\end{array}$ & $N_{L} \times N_{C}$ & $\begin{array}{l}\text { Obtained from bulk calcula- } \\
\text { tion for the conductor super- } \\
\text { cell } \\
\text { Obtained from bulk calcula- }\end{array}$ \\
& $\begin{array}{l}\text { coupling between conductor } \\
\text { and lead R }\end{array}$ & $N_{C} \times N_{R}$ & $\begin{array}{l}\text { tion for the conductor super- } \\
\text { cell }\end{array}$ \\
\hline$H^{C R}$ & & &
\end{tabular}

leads in the simulation cell in order to treat the interface from first principles. The amount of lead layers to be included should converge up to where the local electronic structure of the bulk lead is reached at the edges of the supercell.

Practically the aforementioned steps look like this:

1. Run pwscf to obtain the ground state

pw.x $<$ scf_graphene.in $>$ scf.out

2. Run pwscf to obtain the Bloch states on a uniform kpoint grid.

pw.x < nscf_graphene.in > nscf.out

3. Run pw2want.x to format the pwscf output into the from suitable for WanT input.

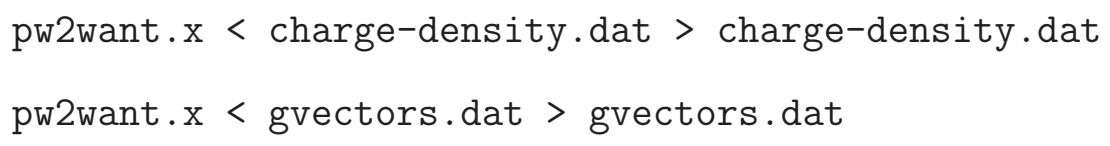

4. Run disentangle to generate a list of the required overlaps and projection inte- 
grals (written into the graphene_WanT.ovp and graphene_WanT.space files). disentangle.x < want_graphene.in > disentangle_graphene.out

5. Run wannier to compute the Hamiltonian matrix and the $U(\mathbf{k})$ (written in the graphene_WanT.ham and graphene_WanT.wan files). wannier.x < want_graphene.in > want_graphene.out

6. Run bands, dos, plot to produce output for vizualization of the disentangles band structure, density of states, and Wannier functions (written in the band_graphene.dat, dos_graphene.dat, and plot_graphene.dat files). band. $\mathrm{x}<$ band_graphene.in > band_graphene.out dos.x $<$ dos_graphene.in > dos_graphene.out plot.x < plot_graphene.in > plot_graphene.out

7. Run conductor to compute the conduction form the Hamiltonian matrix (written in the conductance_graphene.dat file). conductor. $\mathrm{x}<$ conductor_graphene.in > conductor_graphene.out

8. Run current to compute the I-V curve from the conduction function (written in the current_graphene.dat file). current.x < current_graphene.in > current_graphene.out

Steps 6 and 8 are optional. A strict naming convention is adopted during the calculations in order to be able to program all aforementionaed steps in a bash run. sh script:

\$program_name\$_\$job_header\$. in for input files, \$program_name\$_\$job_header\$.out for output log files, \$program_name\$_\$job_header\$.dat for the data output files. 


\section{CHAPTER IV}

Electron microscopy of graphene 
In this chapter we will discuss principal approaches for visual control of graphene atomic structure by means of the Transmission Electron Microscopy (TEM) A comprehensive introduction into the TEM can be found in "Transmission electron microscopy" by David B. Williams, C. B. Carter [67].

In TEM, a beam of electrons generated from either a filament or a field emission gun is focused and accelerated down a column where it passes through a very thin specimen. The resulting interaction of the electrons with the specimen can be used to image and characterize many aspects of the specimen including intensity contrast, phase contrast, defects, interfaces, density variations, atomic composition, crystal structure and orientation, and lattice parameters. A schematic of bright field imaging and parallel beam diffraction in the TEM is shown in Figure 4.1.

Diffraction in the TEM occurs via the same wavelength interference mechanism as in x-ray diffraction. However, the wavelengths of electrons produced by the high acceleration voltages in TEMs are orders of magnitude smaller than the wavelengths of x-rays. Therefore, the angles of diffraction are much smaller, so much so that the planes which produce the diffraction patterns are nearly parallel to the incident electron beam. Additionally, the small specimen thickness is necessary for electron transparency to result in the expansion of the intensity profile of constructive interference about the Bragg angle as there is insufficient material to cause complete destructive interference for angles close to the Bragg angle. This broadening is represented by a broadening of the reciprocal lattice in a direction parallel to the thin direction of the sample. Thus, instead of the reciprocal lattice consisting of points, it can be viewed as an array of rods. These reciprocal lattice rods mean that there will be diffracted intensity from planes slightly off of the exact Bragg condition. Thus, with the specimen being thinner in the $\mathrm{z}$ direction in real space, the reciprocal space dimension lengthens in that direction, creating reciprocal lattice rods. 


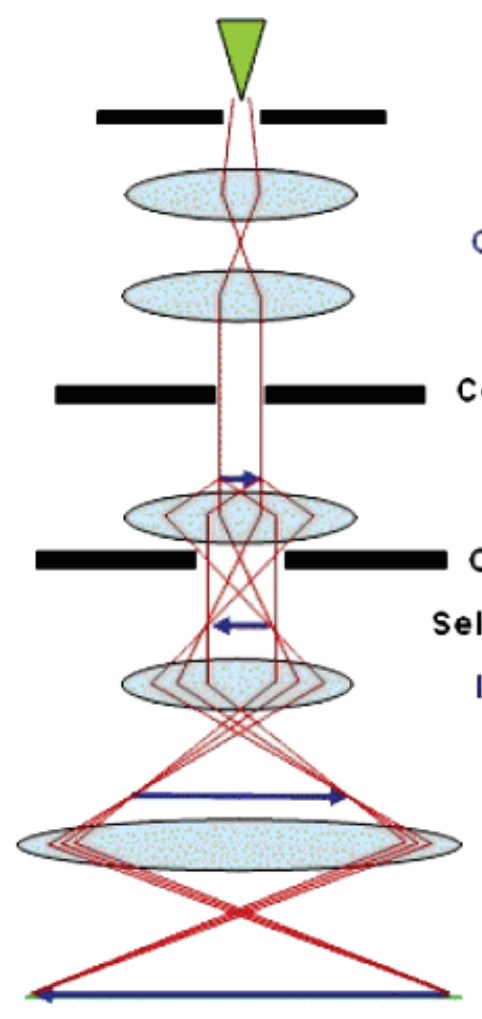

Bright Field Image
Electron source

Anode

Condenser Lenses

Condenser Aperture

Specimen

Objective Lens

Objective Aperture

Selected Area Aperture

Intermediate Lens

Projection Lens

Viewing Screen

Diffraction Spot Pattern

Figure 4.1: Schematic ray path in electron microscope. Adopted from [67].

\subsection{Multislice method}

For high energy electrons, the exchange and correlation between the beam electron and crystal electrons can be neglected, and the problem of electron diffraction is reduced to solving the Schrödinger equation for an independent electron in a potential field. We will follow the ideas of Spence and Zou [68], by discussing the dynamical approach. Starting point is the non relativistic, stationary Schrödinger equation:

$$
\begin{aligned}
& {\left[-\frac{\hbar^{2}}{2 m_{e}} \nabla^{2}+V(\vec{r})\right] \psi(\vec{r})=E \psi(\vec{r})} \\
& \Delta \psi(\vec{r})+\frac{2 m}{\hbar^{2}}(E+V(\vec{r})) \psi(\vec{r})=0
\end{aligned}
$$


Although there are many different ways dealing with the solution of equation (4.1) like the Bloch-waves method, the Howe-Whelan equations, etc. (see e.g. [69]), the multislice method has become the most popular algorithm to simulate TEM images because of its relative simplicity and speed.

The multislice method was first described by Cowley and Moodie [70]. The Fast Fourier Transform (FFT) was added to the algorithm by Ishzuka and Uyeda [71]. The FFT based multislice method as devised by Kirkland [69] will be discussed here.

The multislice method solves this sampling problem by factorizing the electron wave function into a part which varies with the wavelength and into a slowly varying portion that is sampled in real space a at more large sample spacing (because the specimen has only a small effect on the scale of the electron wavelength). The full electron wave function $\psi_{f}$ traveling in the positive $\mathrm{z}$ direction is: $\psi_{f}=\psi \exp (2 \pi i z / \lambda)$ , where $\lambda$ is the electron wavelength and $\psi$ is the slowly varying portion of the wave function. Inserting the above expression for $\psi_{f}$ into the Schrödinger equation 4.1 and solving it for the slowly varying portion of the wave function $\psi$, one concludes that the sampling of the slowly varying portion may be spaced many wavelengths apart. This approximation is justified because the incident electron beam has a relatively high energy and is not changed significantly on the scale of its wavelength. Thus the Schrödinger equation Eq. (4.1) takes form of

$$
-\frac{\hbar^{2}}{2 m}\left(\nabla_{x y}^{2}+\frac{\partial^{2}}{\partial z^{2}}+\frac{4 \pi i}{\lambda} \frac{\partial}{\partial z}+\frac{2 m e V(x, y, z)}{\hbar^{2}}\right) \psi(x, y, z)=0
$$

Dropping the second derivative with respect to $z$ implies neglecting the possibility of the back scattering.

The second approximation in the multislice method is the actual slicing of the specimen as shown in Figure 4.2. The incident wave enters the specimen at the 


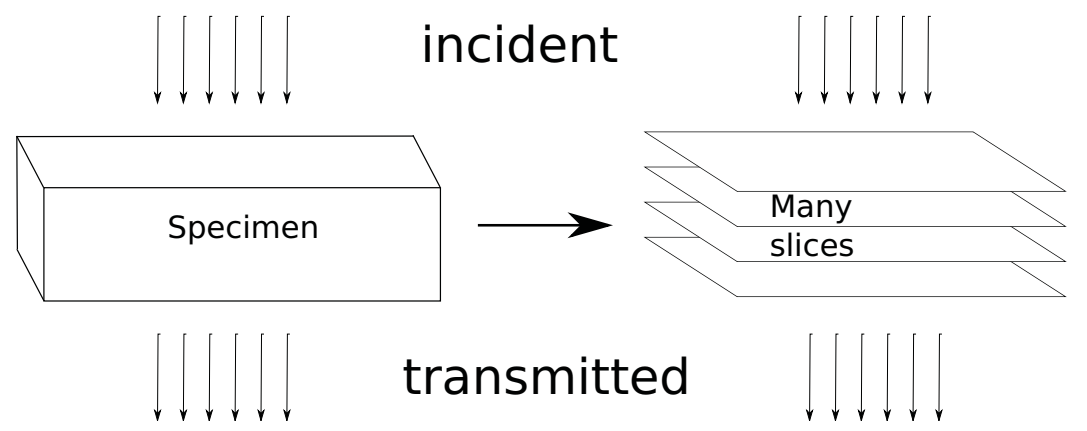

Figure 4.2: The multislice approximation converts a specimen (left) into many thin slices (right)

top, propagates through the specimen and exits at the bottom. In conventional TEM the incident wave is a uniform plane wave and in STEM the incident wave is a small focused probe (at each position in the image). The specimen is divided into many slices, each of which is thin enough to be approximated as a simple phase shift of the electron beam. The electron beam propagates between slices as a small angle of outgoing wave (Fresnel diffraction). The wave is transmitted through a slice of thickness $\Delta \mathrm{z}$ and then propagates a distance $\delta \mathrm{z}$ to the next layer. With some mathematical manipulations of the slowly varying portion of the electron wave function in the Schrödinger equation (4.1) and dropping small terms it yields following multislice equation:

$$
\psi(x, y, z+\Delta z)=p(x, y, \Delta z) \otimes[t(x, y, z, \Delta z) \psi(x, y)]+O\left(\Delta z^{2}\right),
$$

where $\otimes$ represents convolution and $\Delta \mathrm{z}$ is a thin slice of the specimen along the beam direction. The transmission function for the portion of the specimen between $z_{0}$ and $z_{0}+\Delta \mathrm{z}$ is:

$$
t\left(x, y, z_{0}, \Delta z\right)=\exp \left[i \sigma \phi\left(x, y, z_{0}, \Delta z\right)\right]
$$

where $\sigma=2 \pi m e \lambda / h^{2}$ is the interaction parameter, and $\phi\left(x, y, z_{0}, \Delta z\right)$ is the projected 
potential calculated in the direction of the electron wave propagation. The propagator function $p(x, y, z)$ and its Fourier transform $P(\mathbf{k})$ are:

$$
\begin{gathered}
P(k, \Delta z)=\exp \left(-i \pi \lambda k^{2} \Delta z\right) \\
p(x, y, \Delta z)=\mathcal{F}^{-1}[P(k, \Delta z)]=\frac{1}{i \lambda \Delta z} \exp \left[\frac{i \pi}{\lambda \Delta z}\left(x^{2}+y^{2}\right)\right]
\end{gathered}
$$

which is equivalent to Fresnel diffraction (near field) for a distance $\Delta z$. If each slice of the specimen is labeled with subscript $\mathrm{n}(\mathrm{z}=\mathrm{n} \Delta \mathrm{z})$ then the multislice equation becomes:

$$
\psi_{n+1}(x, y)=p_{n}(x, y, \Delta z) \otimes\left[t_{n}(x, y) \psi_{n}(x, y)\right]+O\left(\Delta z^{2}\right)
$$

Convolution requires a number of operations proportional to $\mathrm{N}^{2}$ where $\mathrm{N}$ is the number of points and is still a very time consuming calculation on the computer. However, this form of the equation is very well suited for using the FFT, which is highly efficient and well developed numerical algorithm. The CPU time for the FFT scales as Nlog(N). Then using the Fourier transform, represented as $\mathcal{F}$, the multislice equation becomes:

$$
\begin{gathered}
\psi_{n+1}(x, y)=\mathcal{F}^{-1}\left\{P_{n}\left(k_{x}, k_{y}, \Delta z\right) \mathcal{F}\left[t_{n}(x, y) \psi_{n}(x, y)\right]\right\} \\
\Psi_{n+1}(h, k)=P(h, k) \mathcal{F}\left[t_{n}(x, y) \mathcal{F}^{-1}\left[\Psi_{n}(h, k)\right]\right]
\end{gathered}
$$

The multislice method reduces to a succession of transmit and propagate operations with a fast Fourier transform in between each which is summarized in Figure 4.3. The electron wave function exiting the crystal $\psi_{T}(x)$ is imaged by the objective lens into the CCD image detector. In practice there are several projector lenses after the objective but they can be ignored, because the effects of the objective lens has 


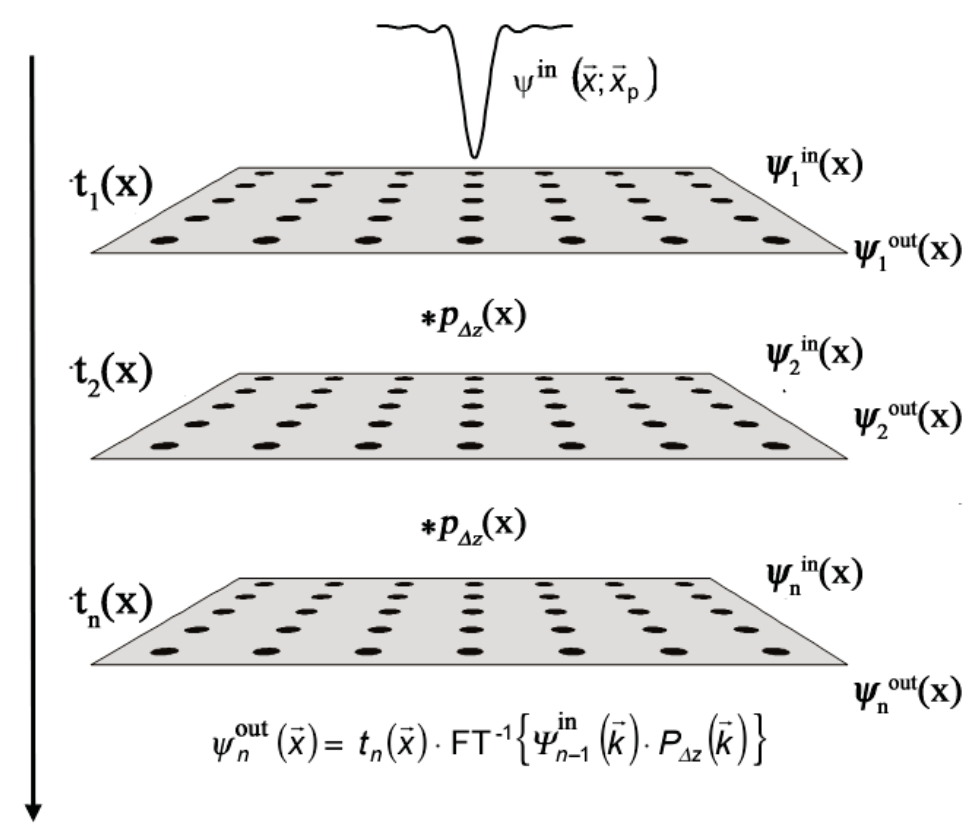

Figure 4.3: Propagation of $\delta$-like wave function through crystal slices in direct space been greatly magnified. The aberrations (mainly spherical and defocus) influence the image as:

$$
g(\vec{x})=\left|\psi_{T}(\vec{x}) \otimes h(\vec{x})\right|^{2}=\left|\mathcal{F}^{-1}\left[\Psi_{T}(\vec{k}) H(\vec{k})\right]\right|^{2}
$$

where $h(x)$ is the point spread function of the objective lens, $\mathrm{x}$ is the position in the image, and $\mathrm{k}$ is the spatial frequency vector conjugate to $\mathrm{x} . \Psi_{T}(k)$ is the Fourier transform of $\psi_{T}(x)$. The convolution is done more efficiently as FFT and the Fourier transform of the point spread function, also known as the contrast transfer function is:

$$
H(\vec{k})=\exp (-i \chi(\vec{k})), \chi(\vec{k})=\frac{2 \pi}{\lambda}\left(\frac{1}{4} C_{s} \lambda^{4} k^{4}-\frac{1}{2} \Delta f \lambda^{2} k^{2}\right)
$$

where $C_{s}$ is the coefficient of spherical aberration, and $\Delta f$ is defocus. 


\subsection{Practical multislice computation}

The calculation algorithm consists of some preparatory steps used to generate the input array for the multislice iterations.

1. Generation of reflections network. The range of the $\{h, k\}$ indexes should not limit the resolution below the resolution of the microscope. Thus the range is determined by the aberration coefficients of the microscope and the electron wavelength. Following Scherzer [72], we defined resolution in the direct space: $d \approx 0.43\left(\lambda^{3} C_{s}\right)^{1 / 4}$, with the maximum scattering angle (resolution in the reciprocal space) $\alpha_{a p}=2^{1 / 2}\left(\lambda / C_{s}\right)^{1 / 4}$. Bragg angles of all relevant $\{h, k, l\}$ are less than $\alpha_{a p}$. This condition limits the number $n$ of Fourier coefficients in the Equation $(4.5)^{1}$.

2. Rotate the crystal. In the case of graphene, however, zone axis was always assumed to be [0001], i.e. along the $c$ axis. This eliminated the need for rotation of the crystal system. Since we concentrated entirely on planar crystals, we limited our consideration to the zero order Laue zone (ZOLZ), i.e. using the Weiss' law set $l=0$.

3. Using the parametrization for atomic scattering factors for neutral atom from the International Tables, Vol C [73] for all elements in the structure, we calculated structural factors by utilizing equation (4.4), where $\left\{x_{i}, y_{i}, z_{i}\right\}$ are fractional atomic coordinates in the corresponding setting and summation runs over

\footnotetext{
${ }^{1}$ For example for $U=100 \mathrm{kV}$ and $C_{s}=3.3 \times 10^{7} \AA$ we obtain $\delta f=1105 \AA, \alpha_{a p}=8.18 \mathrm{mrad}, d=2.75 \AA$. Maximum scattering angle is on order of hundreds of milliradians. So the diameter of pupil in reciprocal space as $R_{a p}=\sin \left(\alpha_{\max }\right) / \lambda \approx \alpha_{\max } / \lambda \sim 3.18 \AA^{-1}$. It means that only reflections with $h^{2}+k^{2}+l^{2}=R_{a p} \cdot a \approx 289$ (if $a=5.350 \AA$ ) should be taken into account. Thus it is enough to scan each Miller index from -17 to 17 to match microscope resolution with the resolution of the simulated images.
} 
all atoms.

$$
F(h, k, l)=\sum_{i} f^{i}\left(\frac{\sin \Theta}{\lambda}\right) \exp \left(-2 \pi i\left(\{h, k, l\} \cdot\left\{x_{i}, y_{i}, z_{i}\right\}\right)\right)
$$

Crystal inner potential was obtained by summing of the Fourier series with the coefficients from (4.4):

$$
V(x, y, z)=\sum_{n} F\left(h_{n}, k_{n}, l_{n}\right) \exp \left(2 \pi i\left(\left\{h_{n}, k_{n}, l_{n}\right\} \cdot\{x, y, z\}\right)\right)
$$

4. Projected potential was calculated by projection along the $z$ axis, i.e. integration over $d z: \phi\left(x, y, z_{0}, \Delta z\right)=\frac{1}{\Delta z} \int_{z_{0}-\frac{\Delta z}{2}}^{z_{0}+\frac{\Delta z}{2}} V\left(x, y, z^{\prime}\right) d z^{\prime}$. Since the $l$ for all reflections from the same LZ are same it was easy to perform integration over $z^{\prime}$; the projected potential for a slice of thickness $\Delta z$ centered on $z_{0}$ is:

$\phi\left(x, y, z_{0}, \Delta z\right)=\sum_{n} F(h, k, l) \operatorname{sinc}(\pi l \Delta z / c) \exp \left(2 \pi i l z_{0} / c\right) \exp (2 \pi i(h x / a+k y / b))$ Thickness and position of each slice was defined by their dependence on the crystal structure.

5. Generated the incident wave function and the propagator sampled on the same grid, and perform iterations as described by the Eq. (4.2). The function was for plane illumination, including the HRTEM mode was initialized as $\psi_{0}(x, y)=1$, which corresponds to the monochromatic plane wave in the reciprocal space.

6. On the last step, objective lens aberrations were applied to the exit wave using equations (4.3a) and (4.3b). 
If iterations in equation (4.2) and convolution in (4.3a) are done using the FFT (unlike the Fourier series summation, which is a very time consuming procedure), following phenomena are known to occur during the computations.

Due to the nature of the algorithm, the transform may not go over usual indices; e.g., in most cases the reciprocal space sampling is from 0 to $\mathrm{M}$ for the FFT routines rather than from $-M / 2$ to $M / 2$. This problem is easily overcome by either using phase factors when evaluating the FFT, thus causing a shift in the reciprocal space or by feeding the reciprocal space wave function into the FFT array in such a way that the problem is not apparent. In the later case the quadrants of the reciprocal space are shifted as shown in figure below.

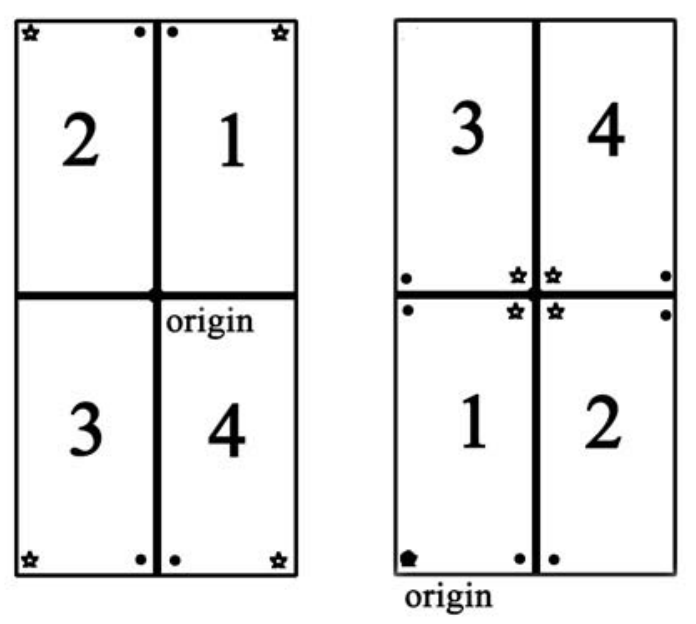

Figure 4.4: Rearrangement of reciprocal space for FFT. Stars and solids indicate relative orientations of quadrants before and after rearrangement

Calculating a convolution in the reciprocal space by use of a Fourier transform involves sampling of the real space wave function. Thus, as a consequence of the sampling theorem [74], the diffraction space wave function appears periodic. The repeat distance 
in reciprocal space is proportional to the inverse of the sampling interval in real space. This enforced periodicity can lead to "wrap around" or "aliasing" effects, where the convolution allows scattering from one cell in reciprocal space to another. In order to avoid this effect the diffracted beams of interest must be embedded into a larger array. When using a phase grating evaluated from Fourier coefficients extending over twice the range of the beams in the calculation, aliasing is avoided when the beams fill only one half the calculation array. If the phase grating has the same number of Fourier coefficients as there are beams, then $2 / 3$ of the calculation array may be filled. The array elements outside this diffraction limit must be set to zero after each iteration step. This is best achieved whilst multiplying by the propagation function. When the number of Fourier coefficients used in phase grating function is the same as the number of beams, the convolution imposes an asymmetric aperture function on each beam. The latter case can lead to false symmetries being present in computed diffractions. The way to avoid this effect is to use Eq. (4.2b) instead of Eq. (4.2a), i.e. perform computations in the reciprocal space.

Because crystal potential is real in the absence of absorption, and the transmission function $t\left(x, y, z_{0}, \Delta z\right)$ by the definition is a pure phase object, i.e. it performs only rotation of complex plane where it is applied but no distortion; and the propagator $P(\mathbf{k})$ is a pure phase object too, the total intensity of beam should be kept on the level of the incident wave function. If we choose to normalize it, then the total intensity after leaving the $\mathrm{n}$-th slice should remain normalized, i.e $I_{n}=\iint_{R}\left|\psi_{n}(x, y)\right|^{2} d x d y=$ 1 for each n. A value of $I_{n}<0.90$ is probably wrong (although it may give a quantitatively valid image) and the sampling should be increased. Values $0.95<$ $I_{n}<1.00$ are typical for well behaved calculations. On the other hand side extreme values like $I_{n}>1.00$ can also occur. Integrated intensity greater than one is a clear indication that the calculation is not correct. 


\subsection{Electron microscopy of graphene}

Image simulations of graphene-based structures were performed using a "homemade" program written in Matlab script language [75]. It allows control of the calculation process based on multislice algorithm at each stage, and hence, provides a better understanding of the image formation on each step.

For convenience, hexagonal atomic structure of graphene was converted into the orthogonal setting by applying the transformation:

$$
Q=\left(\begin{array}{lll}
1 & \overline{1} & 0 \\
1 & 1 & 0 \\
0 & 0 & 1
\end{array}\right)^{-1}=\frac{1}{2}\left(\begin{array}{ccc}
1 & 1 & 0 \\
\overline{1} & 1 & 0 \\
0 & 0 & 2
\end{array}\right)
$$

This transformation allows us to stay in the rectangular coordinate system. In the latter case the hexagonal unit cell with the lattice parameters $a_{h}=2.656 \AA, b_{h}=2.656 \AA$, $c_{h}=6.696 \AA$ is transformed into an orthorhombic one with the lattice parameters $a_{r}=a_{h}=a, b_{r}=4.254 \AA=\sqrt{3} a_{h}, c_{r}=c_{h}=c$. The parameters chosen for the calculations are shown in Table 4.1. The super cell was chosen to be $12 a \times 7 b$, because the ratio $12 / 7$ is a rational approximation of $\sqrt{3}$ - the $a / b$ ratio in the original structure. This choice of the super cell allowed us to keep same resolution in the direct and in the reciprocal space.

The choice of the supercell is important for calculation of the contrast of spatially localized objects on infinite graphene sheet, such as adatom, and functionalizing molecules. Electron waves, scattered by these objects should not interfere with each other and the boundaries in the pixelated image array.

The potential was projected on the planes 0 and $0.5 c$, where the thickness of 
Table 4.1: The resolution and extent in real and reciprocal space

\begin{tabular}{|l|l|l|}
\hline$N_{x}, N_{y}$ & Array dimensions & $512 \times 517$ \\
\hline$n_{x}, n_{y}$ & Number of unit cells & $12 \times 7$ \\
\hline$X=n_{x} a, Y=n_{y} b$ & Real-space array extent & $29.47 \AA \times 29.77 \AA$ \\
\hline$X / N_{x}, Y / N_{y}$ & Real-space array resolution & $0.0576 \AA \times 0.0576 \AA$ \\
\hline$\lambda N_{x} / X, \lambda N_{y} / Y$ & Reciprocal-space array extent & $725 \mathrm{mrad} \times 725 \mathrm{mrad}$ \\
\hline$\lambda / X, \lambda / X$ & Reciprocal-space array resolution & $1.402 \mathrm{mrad} \times 1.416 \mathrm{mrad}$ \\
\hline$\alpha_{\max }=\frac{\lambda}{3} \min \left[\frac{N_{x}}{X}, \frac{N_{y}}{Y}\right]$ & Maximal included scattering angle & $242 \mathrm{mrad}$ \\
\hline$k_{\max }=\min \left[\frac{N_{x}}{2 X}, \frac{N_{y}}{2 Y}\right]$ & Cut-off aperture to prevent aliasing & $8.68 \AA^{-1}$ \\
\hline
\end{tabular}

each slab was $c / 2$. All functions (i.e. the transmission function, the propagator and the initial wave function) were sampled at the same sample rate as the projected potential. According to the common practice of avoid aliasing, a $0.35 k_{\max }$ aperture was applied to all functions in the reciprocal space.

Two major types of aberrations were included into the calculation: spherical and chromatic. However, since we used a microscope with a spherical aberration correction, the $C_{s}$ was usually set to zero, unless we wanted to emphasize the change if the spherical aberrations are included. In contrast, chromatic aberrations were usually taken into account. The coefficient of chromatic aberration was set to $C_{c}=1.3 \mathrm{~mm}$, acceleration voltage instability was $\Delta E=0.6 \mathrm{eV}$. Beam divergence angle was taken as $\alpha=0.1 \mathrm{mrad}$.

\subsubsection{Multilayer stacking}

The HRTEM contrast of graphene film depends on the defoci and other imaging conditions as well as on a stacking sequence.

Bilayer graphene can exist in two different polytypes: the AA stacking (Fig. 4.5a) 
and the conventional graphite-like AB stacking (Fig. 4.5b). In the AA-stacking each carbon atom is located exactly above the one underneath. In the AB-stacking, next layer is shifted verses the previous one by the translation vector $t=0.5 a_{h}+0.5 b_{h}$. This stacking sequence is the common stacking sequence of multilayer graphite. The AA-stacking just repeats the same arrangement of the layers. The space groups and atomic coordinates of both stacking sequences are the following: $\mathrm{AB}$ : $C m c 2_{1},(0,0,0)$, $(0,1 / 3,0)$ AA: $C m c m,(0,1 / 3,0)$.

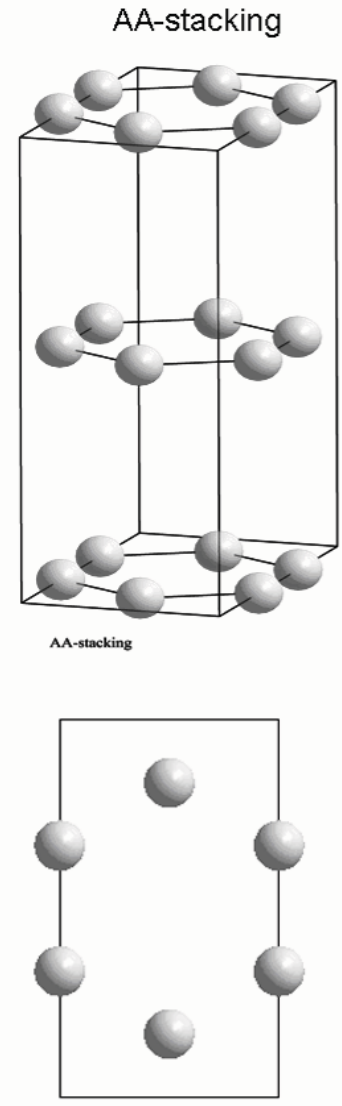

(a)
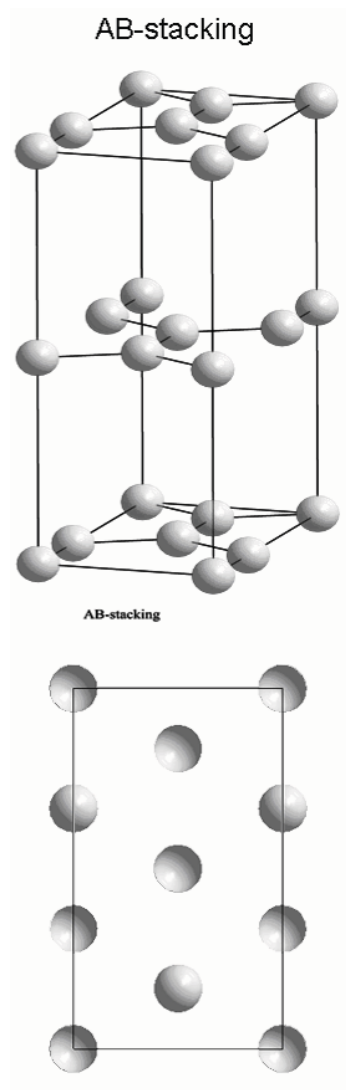

(b)

Figure 4.5: Crystalline models for AA- (a) and AB-stacking (b) in graphene. Perspective and top views

Figure 4.6 shows the effect of defocus on HRTEM contrast of a single and bi-layer graphene based on computer simulations. One can see that carbon atomic positions 
could be resolved in single-layered graphene films (see contrast patterns of the top row of Fig. 4.6) while it is challenging in case of bi- (contrast patterns of the third row) and multi-layered graphene (contrast patterns in the forth and fifth rows).

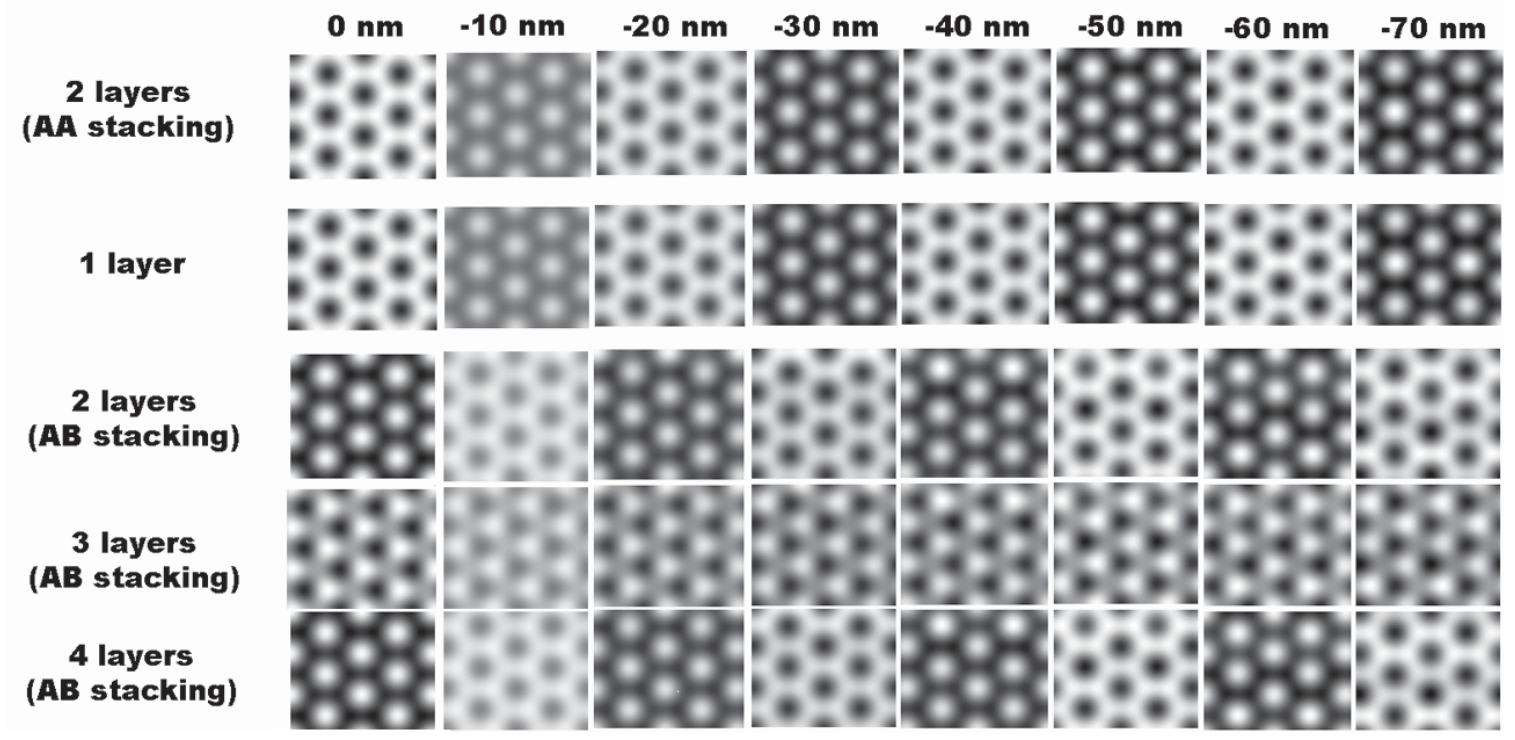

Figure 4.6: Computer simulations of HRTEM contrast of single- and bi-layer graphene (the first and second rows, respectively) at defoci of 0 and $-70 \mathrm{~nm}$ (the left and right columns, respectively)

One can see from Fig. 4.6 that in the case of AA stacking sequence (Fig. 4.5a), HRTEM contrast does not substantially change with the number of layers except with some contrast enhancement. In other words, there is no visible change in the contrast pattern by adding the second graphene layer. Contrast is calculated using the common formula $C=\frac{I_{\max }-I_{\min }}{I_{\max }+I_{\min }} 100 \%$. A single layer of graphene yields contrast of 0.0093 , triple layered 0.0272 , five layered 0.0441 . These values correspond to the squared exit wave after all aberrations are applied, however the CCD cameras possess a contrast enhancement feature, allowing the "stretch out" of this minuscule difference in the minimum and maximum intensity in a such way that it covers the whole grayscale range from 0 to 255 .

The limitation of this method of contrast enhancement is the signal to noise ratio. 
The noise intensities are stretched out together with the contrast, limiting the possibility to observe small contrast variations. The impact of noise on the final images can be substantially reduced by applying conventional signal processing techniques, such as low- and high-pass filtering, masking, windowed Fourier transformation. The noise was not included into the simulations, because we are interested in semi-qualitative interpretation on contrast only.

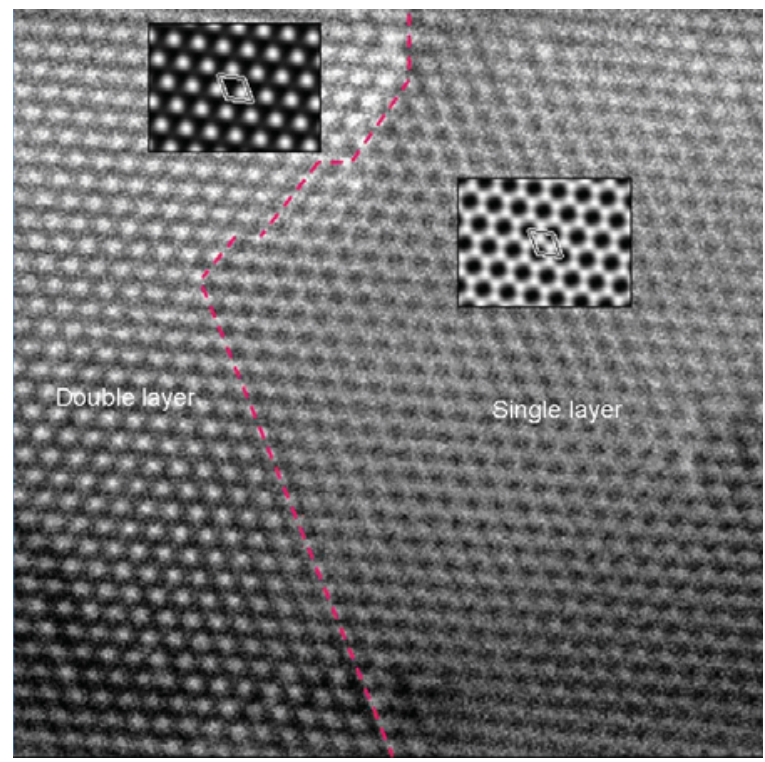

Figure 4.7: HRTEM image of graphene taken on FEI Titan on the edge of a film. The imaging conditions were set close to Schertzer focus. The image contains areas of single and bi-layer graphene.

Figure 4.7 shows the HRTEM image of the graphene film near the edge that contains areas of single and bi-layer graphene. The image was taken at Scherzer focus with a very short exposure to prevent radiation damage and avoid image drift, therefore, it has relatively low signal-to-noise ratio and poor contrast. To increase the signal-to-noise ratio and enhance the contrast, the original image was 512x512 pixel in size and superimposed with the filtered copy of the same image. For filtering, a low-pass filter in the Fourier-space has been used. This allows one to enhance the contrast while keeping the major contrast features of the original image visible. The 
contrast within each region is not uniform probably due to crumpling of the layer and variations in its local orientations with respect to electron beam. One can also see the structural defects, domains of different contrast pattern and grain boundaries between these domains (marked by dotted line). Inserts are images of two different contrast patterns prepared by the crystallographic image processing software [76] to enhance the contrast. The contrast of carbon atoms in the area to the left is white while it is black in the adjacent region to the right. Based on the contrast simulation shown in Fig. 4.6, the area to the right is a single-layer graphene film and the adjacent area (to the left) is bi-layer region of inverted contrast. The contrast inversion has been observed earlier by other researchers in aberration corrected HRTEM (see e.g. [77]). Thus, the dotted line is the boundary between the single and bi- layer regions being also the edge of the second layer on top of single layer film.

The contrast interpretation for the AA-stacking is straightforward, unlike the natural AB-stacking, since the qualitative contrast in the AA case is affected by the defocus value only but not by the number of the layers. At the same time, in case of the AB stacking, the opposite occurs where the contrast significantly changes by adding another graphene layer: one can see the contrast inversion from a single layer in Fig. 4.8a to a bi-layer in Fig. 4.8b, in agreement with experimental observation. On the other hand, adding more layers to the AB stacking would not lead to a drastic contrast change: one can see the inability to distinguish between every second layer of graphene under any defocus. The trigonal arrangement shown in Fig. 4.8c repeats to the next nearest layers with the exclusion of the first one.

Figure 4.8 shows the effect of the number of graphene layers (from one to four) of AB-stacking on HRTEM contrast. Atomic positions are marked by dots of different tone. The atoms in the first, second, third and fourth sub-layers are shown in the dots of following tones $\square \square \square$ respectively. The dots on the picture slightly deviate 


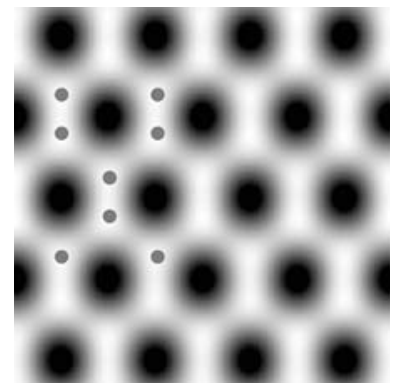

(a)

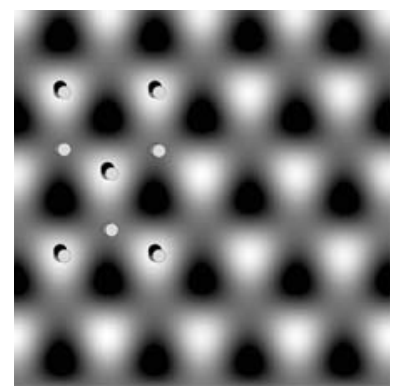

(c)

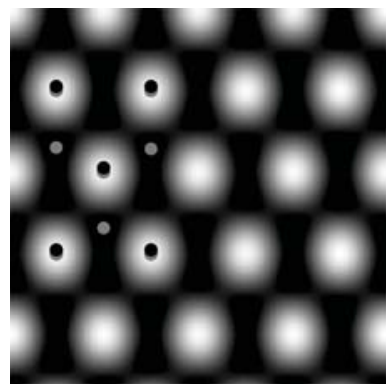

(b)

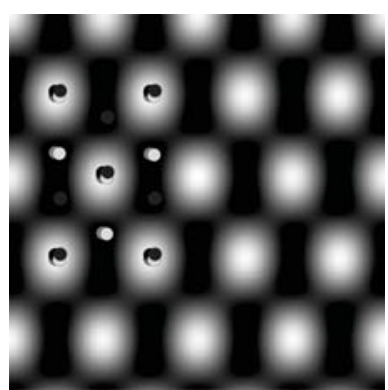

(d)

Figure 4.8: Computer simulations of HRTEM contrast of single- (a), bi- (b), tri- (c) and tetra (d)-layer graphene at defocus of $-60 \mathrm{~nm}$. Contrast on the simulated images has been maximized and, therefore, looks much higher as compared to experimental images of Fig. 4.7 
from the actual position of the carbon atoms in order to avoid overlapping. One can see that carbon atomic positions could be resolved in single- layer graphene films while this may be challenging in case of bi- layer and mulilayer graphene.

Depending on the number of the atoms in a single atomic column relative to the neighbouring columns, its contrast manifests itself in a different way. By adding a second layer (Fig. 4.8b) the columns located at the vertices and in the center of the rhombic graphene cell contain twice as many atoms as the columns within the cell, causing apparent inversion of the contrast. By adding one more layer one obtains a trilayed graphene. In this structure atomic columns at the vertices and in the center of the cell still remain the densest ones, however the remaining columns differ by the number of atoms in them and thus the contrast changes too: the $(0,2 / 3)$ column now has two atoms, whereas the $(0,1 / 3)$ column only one. Adding the fourth layer removes the difference between the $(0,1 / 3)$ and $(0,2 / 3)$ atomic columns, repeating the bilayer arrangement.

\subsubsection{Graphene Nanoribbon (GNR)}

The dotted line in Fig. 4.7 is the boundary between the single and bi- layer regions being also the edge of the second layer on top of single layer film. This edge of the second layer seems to be mainly of a zigzag configuration in agreement with earlier observations. Indeed, Girit et al. [78] observed the structure of the holes in graphene membrane where the most prominent edge structure appeared to be of the zigzag type, with the bottom and lateral sides of the hole aligned in the zigzag directions of the lattice. These authors proposed a simple model to explain the stability of zigzag edges observed both experimentally and with simulations by considering the effect of ejecting an atom at the edge for each chirality. Our observations suggest that the zigzag configuration seems to be more stable also for the edge of second layer of the 
bilayer region.

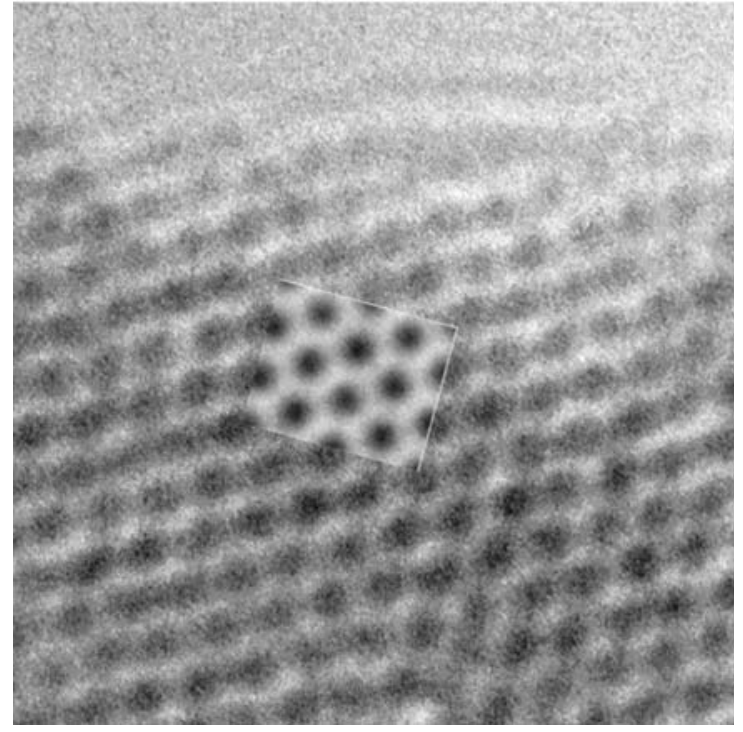

(a)

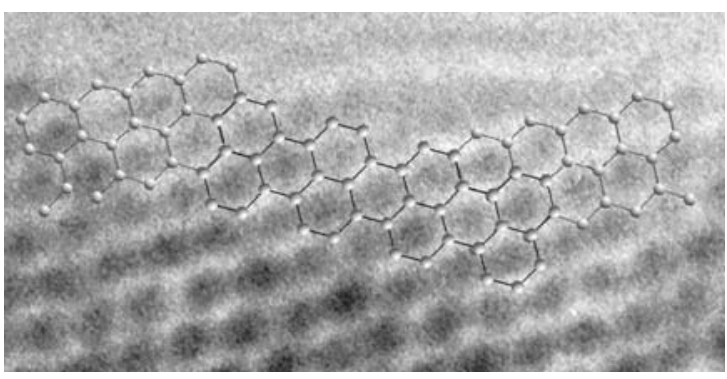

(b)

Figure 4.9: HRTEM images of the edge of the graphene film: (a) raw image and (b) fragment of the same image with stick-and-ball atomic models overlaid on the edge.

Figure 4.9 shows the HRTEM image of graphene film at the edge demonstrating a nontrivial contrast due to uneven cut. Both the "zigzag" and "armchair" arrangements are seen at the edge of the single graphene film. Imaging conditions were: $U=80 \mathrm{kV}, C_{s}=1.2 \mathrm{~mm}, C_{c}=1.3 \mathrm{~mm}, \Delta f \sim 0$. The inset on Fig. 4.9a is a simulated image of a single layered graphene, when the simulation conditions are same as the imaging conditions used in the experiment. Opacity of the inset is set to $50 \%$ for clarity. The structure of the edge consists of the mixture of "zigzag" and "armchair"like atomic arrangements. Their mutual orientation differs by $30^{\circ}$. The contrast of the graphene film with zigzag and armchair edges was analyzed using computer simulation. (Fig. 4.10)

For the calculation of the contrast of graphene edges, we used ribbons with two different atomic arrangements, zigzag and armchair, and a larger unit cell. The atomic structure models were selected in such a way that the distance between the 

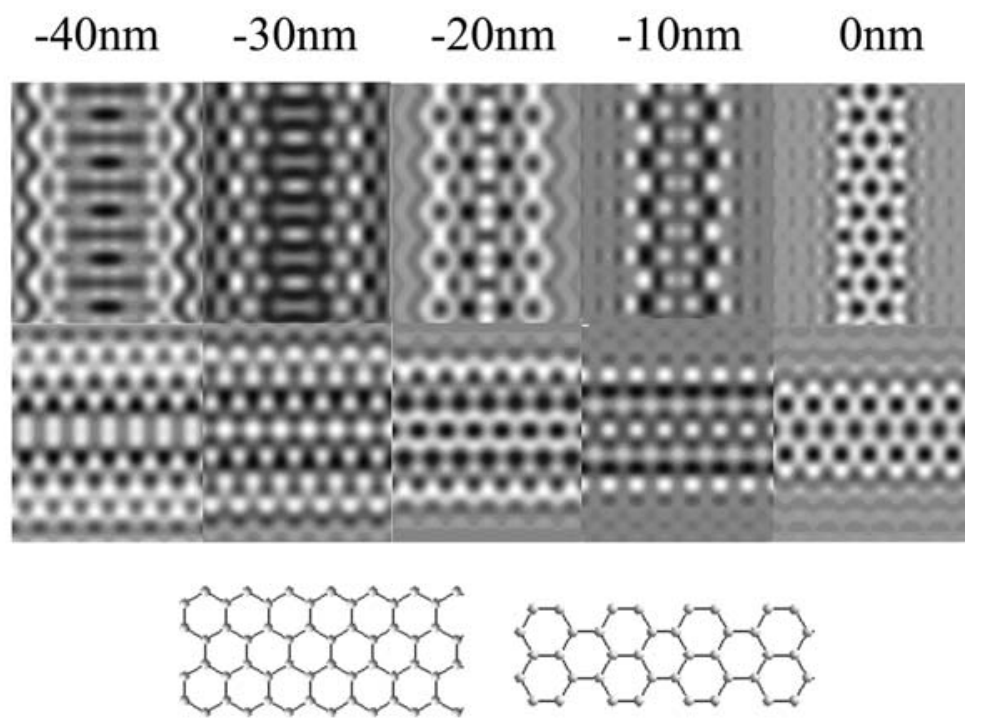

Figure 4.10: Computer simulations of HRTEM contrast of the "zigzag" (right) and the "armchair" (left) ribbons of single graphene layers. Imaging conditions: $U=$ $80 \mathrm{kV}, C_{s}=0, C_{c}=0$, defocus values are shown on the left.

ribbons stayed wide enough to avoid interference between the neighbouring ribbons. The ribbons were periodic in one dimension, thus there was no need to keep the cell length long in the direction along the only translation. (Crystalline ball-and-stick models are placed on the left for comparison.)

Using certain techniques [79], graphene films can be cut along lines with either zigzag or armchair edge configurations. The ribbons exhibit Fresnel-like contrast from the edges depending on the focus values shown in Fig. 4.10. Experimental image of Fig. 4.7 shows uneven Fresnel-like contrast at the edge of the grapheme film because the edge was uneven and some parts of the film may be bent.

The contrast is strongly influenced by the Fresnel-fringes, thus, it would impose challenges in observation of the ribbons in a conventional (non aberration corrected) microscope. In order to simulate the influence of a non-ideal TEM, the apparatus function was applied to the electron wave after its propagated through the sliced crystal. The apparatus function includes experimental parameters of the microscope 
such as chromatic aberration, beam divergence, and acceleration voltage instability. The spherical aberration coefficient was set to zero, since the microscope used was equipped with a $C_{s}$-corrector.

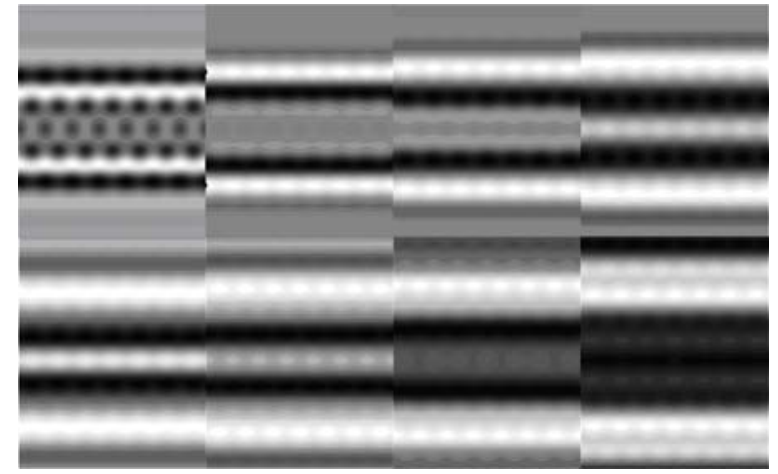

(a)

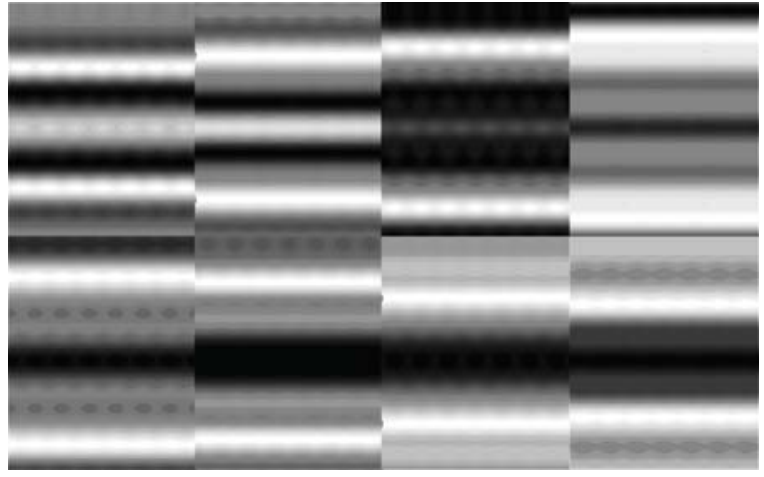

(b)

Figure 4.11: Computer simulations of HRTEM contrast of the armchair ribbon. (a) Only chromatic aberration included. (b) Both spherical and chromatic aberration are included. Defocus series (a) top row $\Delta f=0,-10 \mathrm{~nm},-20 \mathrm{~nm},-30 \mathrm{~nm}$, bottom row, $\Delta f=-40 \mathrm{~nm},-50 \mathrm{~nm},-60 \mathrm{~nm},-70 \mathrm{~nm}$, (b) top row: $\Delta f=0-4 S c h$, bottom row $\Delta f=5$ Sch -9 Sch.1Sch $=-16.9 \mathrm{~nm}$

Thus a very careful choice of the microscope settings is important for the contrast interpretation. For the simulated images below the coefficient of chromatic aberration was set to $C_{c}=1.3 \mathrm{~mm}$, acceleration voltage was $\mathrm{U}=80 \mathrm{kV}$, acceleration voltage instability was $\Delta E=0.6 \mathrm{eV}$, and the beam divergence angle was taken as $\alpha=0.1$ mrad. We also analyzed the effect of two major aberrations of the objective lens, spherical and chromatic, on the contrast. Because the experimental images were taken with a spherical aberration correcter, the $C_{s}$ was usually set to zero. In contrast, the chromatic aberration was usually taken into account.

To emphasize the importance of spherical aberration on the contrast formation, the spherical aberration was included in some calculations. From the HRTEM contrast simulations (Figs. 4.10 and 4.11) one can see that the contrast does not vanish under the Gaussian as well as under Scherzer illumination conditions (Fig. 4.11b) if 
both types of aberrations are present in the microscope. However, if $C_{s}=0$ one can still resolve the atomic structure at $\Delta f=0$, even if chromatic aberrations are not totally compensated.

\subsubsection{Adatoms}

Adsorbats and impurities are typically present on the surface of the graphene films due to processing and handling and especially due to sample preparation for TEM. Presence of adatoms on the graphene surface results from the exceedingly high reaction ability of graphene and a trend to saturate dangling $\pi$-bonds by adsorbing atoms from the reaction mixture or the air. An extended theoretical study of stability of different small molecules in graphene surface was undertaken by Erni et al. in [80]. The authors have considered molecules forming covalent bonding with one carbon atom, i.e. located in the B position (see below). However, it is known $[81,82]$ that different molecules may be attached to different sites with different symmetry, and discrimination between those sites is sometimes a challenging task for miscroscopists.

For calculation of contrast from adatoms we modified the crystal structure so that the whole graphene supercell was now considered as a single unit cell in the P1 group with the lattice parameters $a=17.192 \AA, b=21.269 \AA, c=6.696 \AA$ and the adatoms were placed in the A,B,C positions (see Fig.4.12). The choice of the super cell was dictated by the necessity to avoid interference of the electron waves scattered by adatoms from one cell with those from another cell, i.e. maintain a proper distance between them.

Simulated contrast from a Fe-adatom in various positions (A, B, C) (see Fig. 4.13 a,b,c) under various defoci $(0,-10,-20,-30 \mathrm{~nm}$ for top row and $-40,-50,-60,-70 \mathrm{~nm}$ for bottom row) demonstrates that the symmetry of the contrast pattern reflects the symmetry of the atomic arrangement associated with position of the foreign atom in 


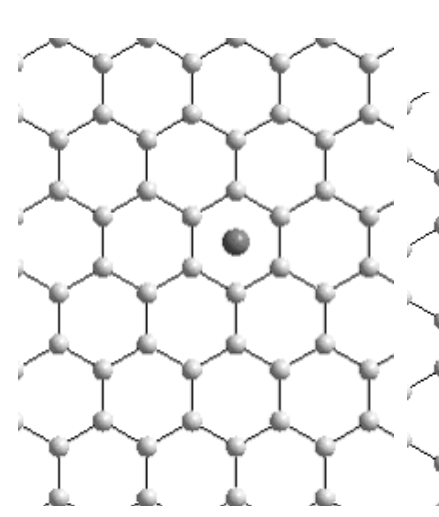

(a)

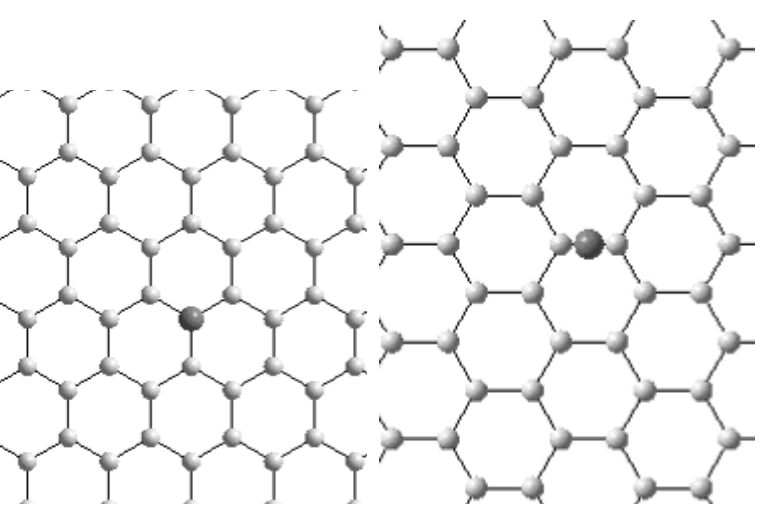

(b)

(c)

Figure 4.12: Crystal structure of a graphene sheet and an adatom in the A (a), B (b) and $\mathrm{C}(\mathrm{c})$ position

crystal lattice of graphene film.

This provides a way to determine the position of the adatom on the graphene surface by the analysis of the symmetry of the contrast pattern. Indeed, an adatom in the A position yields 6-fold contrast, that is in correspondence with the sitesymmetry of the defect. An adatom in the B position gives trigonal contrast, and in the $\mathrm{C}$ - 2-fold. In fact the contrast from the adatom totally disappears at defoci of more that -200nm since the size of the electron probe becomes much larger compared to the typical size of the structural feature. In case of a non-aberration corrected microscope we conclude that contrast cannot be directly interpreted at all. If an adatom is an atom of a light element than the qualitative contrast remains almost same, whereas the total contrast of the image drops further.

\subsubsection{Functionalizing molecules}

Functionalization of graphene changes its electronic properties dramatically. These include, but are not limited to bandgap, electron mobility, and absorption coefficients. Also the degree of alteration depends on the concentration of functionalizing 


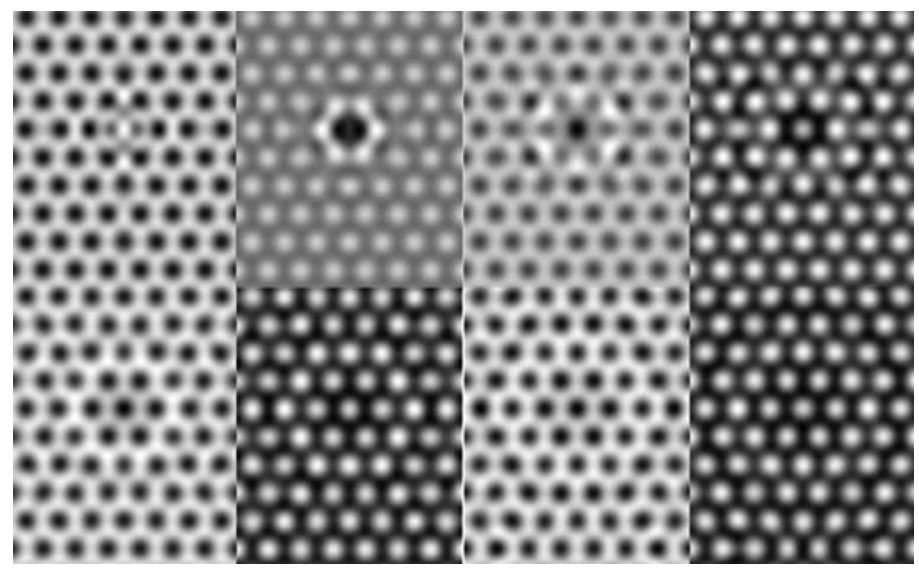

(a)

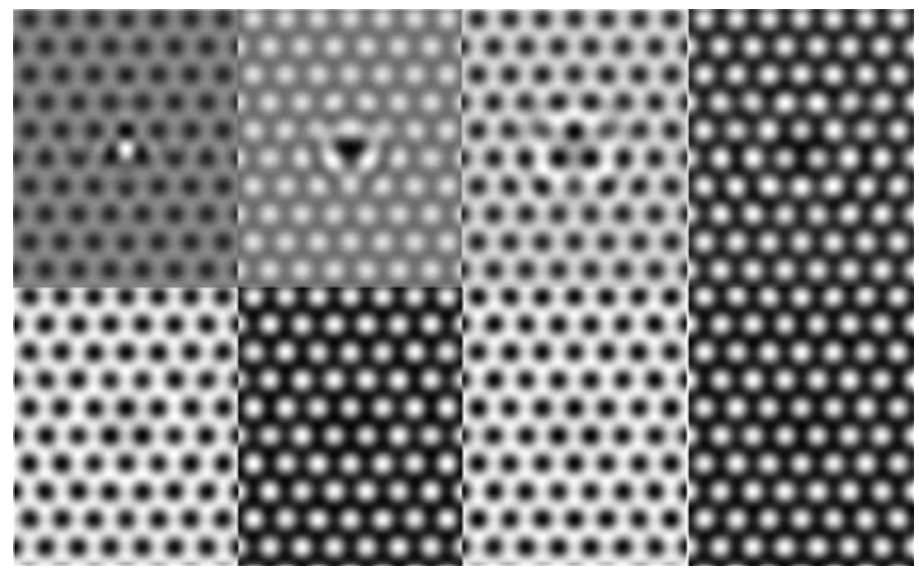

(b)

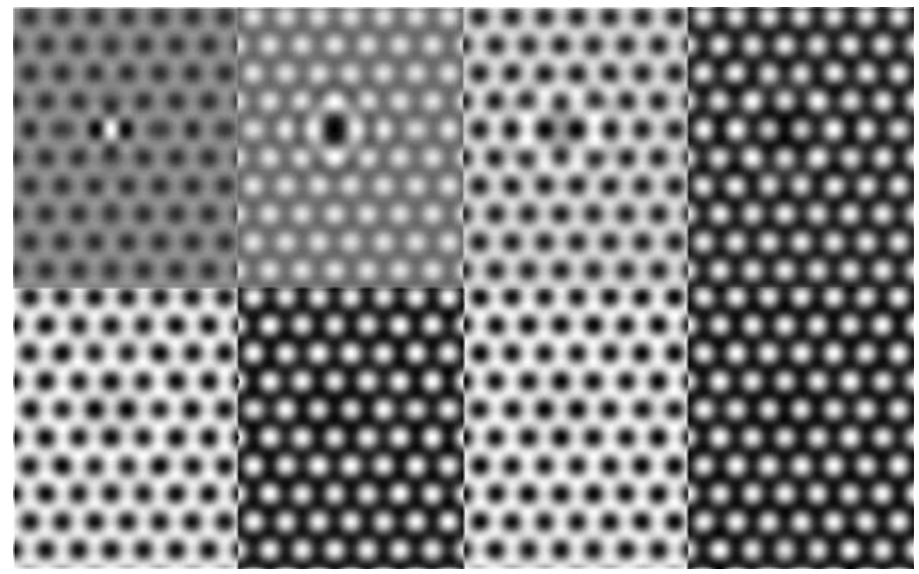

(c)

Figure 4.13: (a) Contrast for an iron adatom in the A position, (b) Contrast for an iron adatom in the $\mathrm{B}$ position, (c) Contrast for an iron adatom in the $\mathrm{C}$ position. 
molecules. Therefore it becomes an important problem to estimate the degree of functionalization of graphene. The only tool that would allow us to do so is the TEM. In this section simulated TEM images of PFPA, MBA $\left(\mathrm{M}\left[\eta^{6}-\mathrm{C}\right]\left(\mathrm{C}_{6} \mathrm{H}_{6}\right)\right)$, and $\mathrm{MCO}$ $\left(\mathrm{M}\left[\eta^{6}-\mathrm{C}\right](\mathrm{CO})_{3}\right)$ functionalized graphene will be presented along with the discussion of the factor that impedes our ability to obtain a self-explanatory image of molecules on top of the graphene surface. The choice of PFPA, MBA, and MCO is dictated by further studies of their structural and electronic properties in chapters VI and VII. The atomic structure of functionalized graphene was obtained by means of the geometry optimization procedure, which searches for the atomic configuration that leads to the energy minimum of the structure. The details of this procedure are discussed in chapters V, VI, and VII.

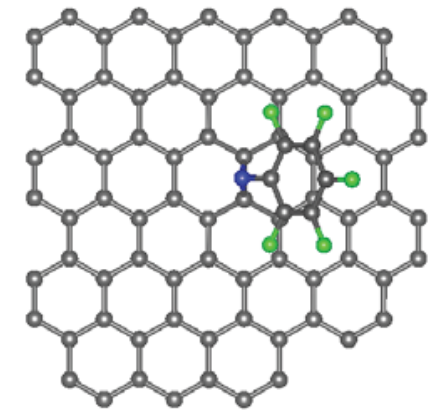

(a) PFPA

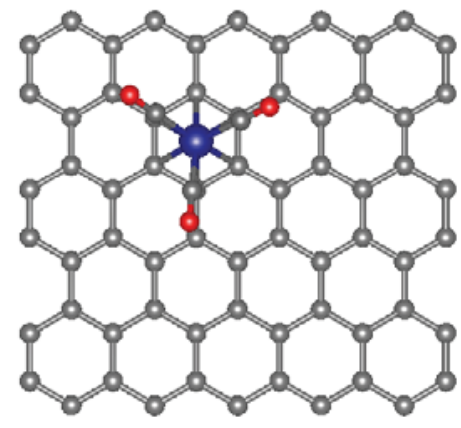

(b) $\mathrm{MCO}$

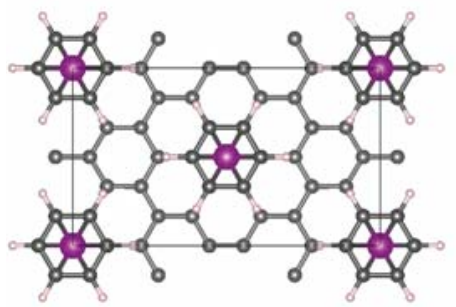

(c) MBA

Figure 4.14: Atomic structures of functionalized graphene. (Top view). PFPA-FG (a), MCO-FG(b), MBA-FG(b)

The atomic structures, selected for simulations are depicted in Fig. 4.14. The number of functionalizing molecules is the same as in chapters VI and VII, i.e. one molecule per 6x6 hexagonal graphene cell. However, for the same reasons as discussed, the structure the structure was converted into the orthorhombic setting using Eq. (4.6). After a single slice electron wave propagation the following pictures were obtained for spherical aberration corrected imaging conditions (See Fig. 4.15). Under 


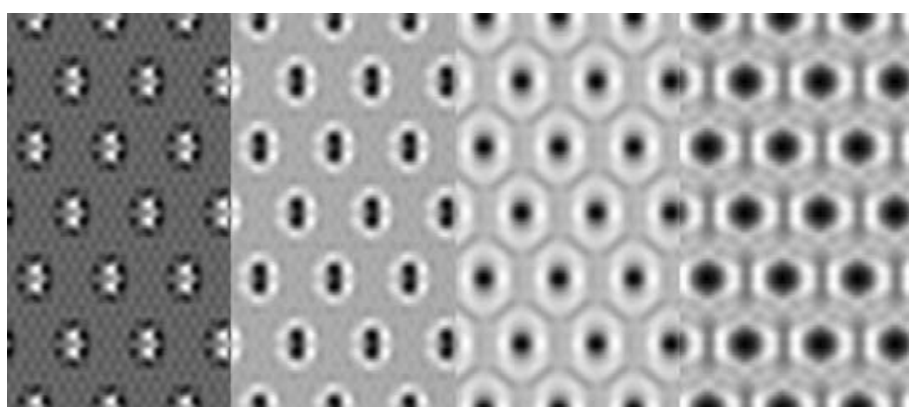

(a)

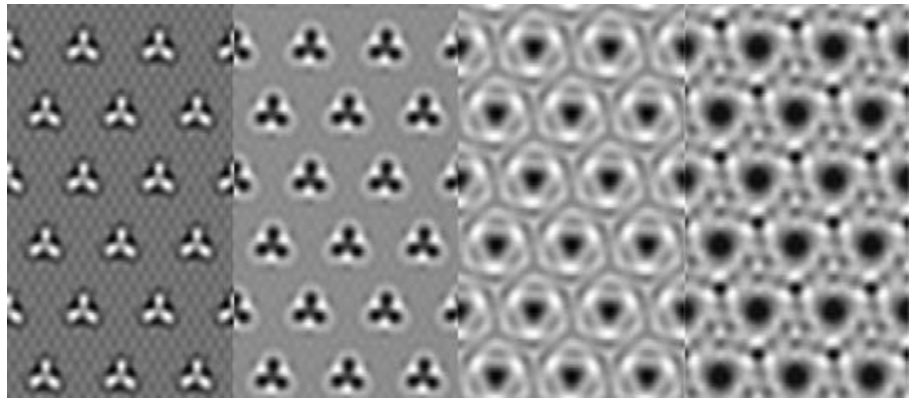

(b)

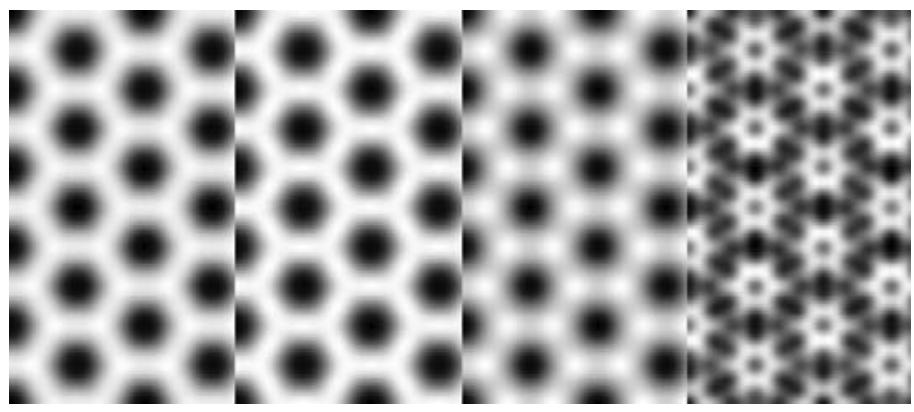

(c)

Figure 4.15: TEM contrast for PFPA-FG (a), MCO-FG (b), and MBA-FG (c). Defocus values are $-0 \mathrm{~nm},-15 \mathrm{~nm},-45 \mathrm{~nm},-75 \mathrm{~nm}$ (from left to right), $\mathrm{U}=80 \mathrm{kV}, \mathrm{Cc}=1.3 \mathrm{~mm}$, $\mathrm{Cs}_{\mathrm{S}}=0$

the aberration corrected imaging conditions, the TEM contrast preserves the symmetrical features of the molecules very well, i.e. 2-fold symmetry for the PFPA molecules, 3-fold for the MCO, and 6-fold for MBA. It is worth pointing out that the rotational barrier for MBA and MCO molecules is about $0.5 \mathrm{eV}$ and thus they are not expected to rotate freely at room temperature. However, in all cases they act as rigid rotators, 
and thus the symmetry can still be revealed even under high temperature conditions.

Spherical aberration also preserves symmetrical peculiarities, however, in this case direct interpretation of the TEM images may be quite problematic. Figure 4.16 depicts simulated TEM contrast for nonaberration corrected $\left(C_{s}=1.2 \mathrm{~mm}\right)$ imaging conditions.

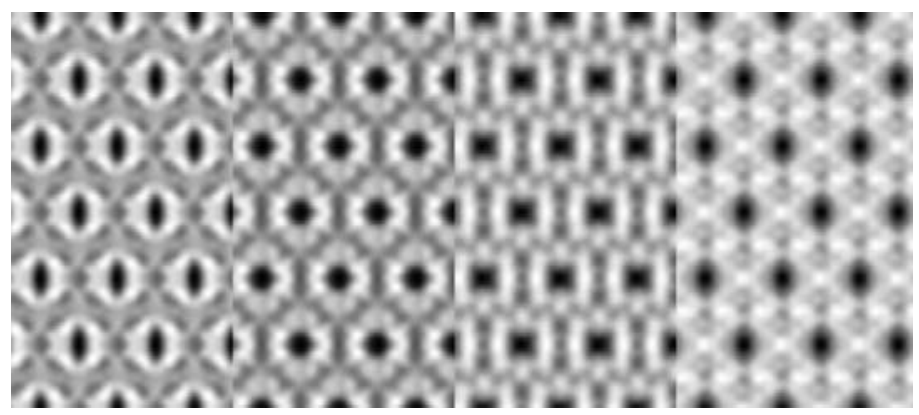

(a)

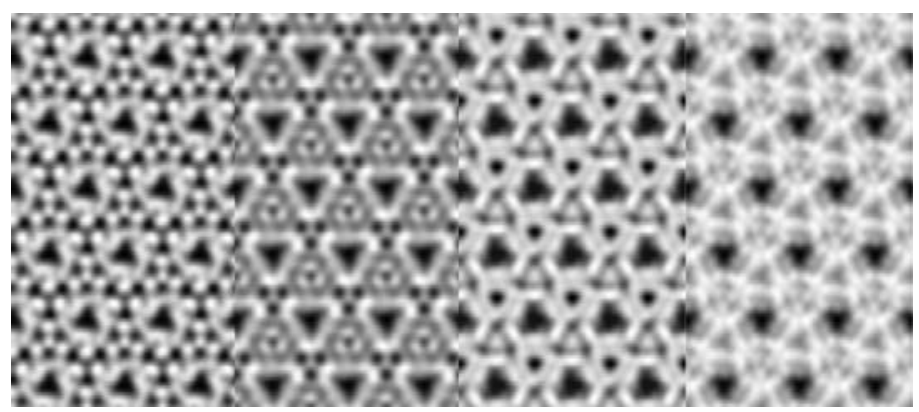

(b)

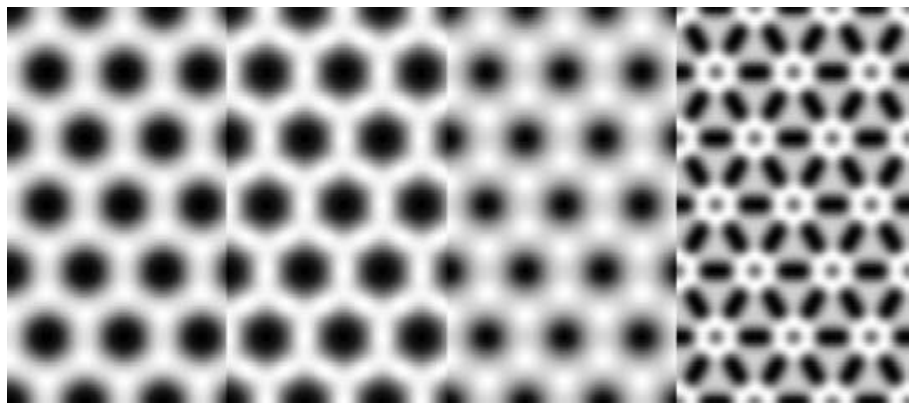

(c)

Figure 4.16: TEM contrast for PFPA-FG (a), MCO-FG (b), and MBA-FG (c). Defocus values are $-0 \mathrm{~nm},-15 \mathrm{~nm},-45 \mathrm{~nm},-75 \mathrm{~nm}$ (from left to right), $\mathrm{U}=80 \mathrm{kV}, \mathrm{Cc}=1.3 \mathrm{~mm}$, $\mathrm{Cs}=1.2 \mathrm{~mm}$ 
From both ideal (Fig. 4.15) and aberrated imaging conditions (Fig. 4.16) it follows, that PFPA and MCO structures still yield quite distinct patterns that cannot be confused with the contrast from pristine graphene, but the contrast caused by MBA functionalization can hardly be segregated from the substrate contrast. The reason for that is exactly matching symmetry of underlying graphene sheet and the symmetry of the top benzene ring. Therefore the degree of MBA functionalization can not be obtained form TEM solely.

The contrast produced by functionalizing molecules is strongly influenced by local symmetry of the molecules and the defocus values. Noncorrected spherical aberrations make it almost impossible to observe individual molecules, however they still preserve the local environment. Electron waves, scattered off the MBA molecules and underlying graphene sheet produce an interference pattern almost totally obscuring the presence of the functionalizing groups. Also very minor difference in scattering power makes it unable to distinguish between e.g. $\mathrm{MCO}$ and $\mathrm{MCN} \mathrm{M}\left[\eta^{6}-\mathrm{C}\right](\mathrm{CN})_{3}$ molecules. These simulations provide a route to control the concentration of functionalizing molecules, which is crucial for understanding electronic properties of functionalized graphene. 


\section{CHAPTER V}

Electronic properties of pristine graphene and graphene nanoribbons 
In this chapter the electronic structure of pristine graphene and graphene nanoribbons are discussed. The algorithms and approximations used for these calculations are discussed in details in this chapter on a relatively simple example of pristine graphene and then move on to more complicated structures. Special emphasis is placed on electric and heat conductance of graphene nanoribbons due to their practical application as interconnects in ICs.

\subsection{Band structure of pristine graphene.}

As it follows from the quantum conductance equations, outlined in section 3.2 , electron dispersion relation (aka band structure), plays crucial role for understanding of the electron transport in nanostructures. The band structure represents the energy levels as the solutions of the Schrödinger equation for different k-points. Usually the band structure is plotted along a high symmetry direction in the BZ. This conventional representation of the band structure is a $3 \mathrm{D}$ cross section of the full $4 \mathrm{D}$ (3D BZ plus one dimension for the energy) picture. Since graphene is a 2D material, its BZ is flat and the band stricture can be rendered in a $3 \mathrm{D}$ representation.

\subsubsection{Tight-binding model}

As it was already mentioned in Chapter I, first theoretical attempt to calculate graphene's band structure was undertaken by Wallace in 1947 [8]. His original approach will be briefly outlined here, since it is important for understanding the

reasons why any functionalization leads to opening of the band gap between the valence and the conductive bands. Within the tight-binding approximation, a solution $\psi(r)$ to the time-independent single electron Schrödinger equation is approximated as a linear combination of the wave functions, belonging to different sublattices $\phi(r)$ 


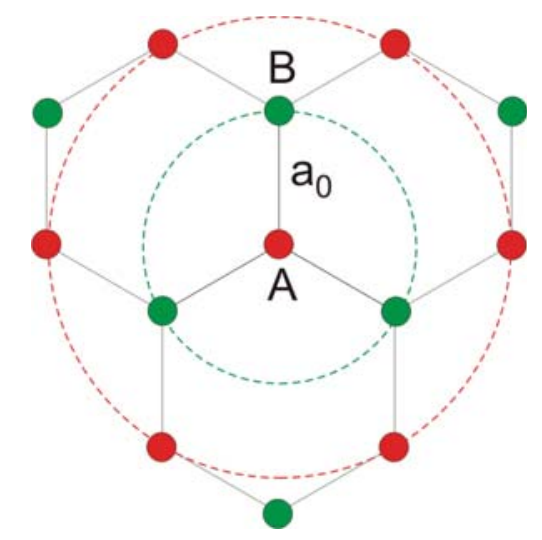

Figure 5.1: Nearest neighbors in the coordination environment of the central carbon atom (A). Red dashed line corresponds the the atoms belonging to the same sublattice as $(\mathrm{A})$; green dashed line corresponds to the atoms from the second sublattice (B).

as $\psi(r)=c_{1} \phi_{1}(r)+c_{2} \phi_{2}(r)$, where the coefficients $c_{1}$ and $c_{2}$ are determined from the solution of the secular equation. Functions $\phi(r)$, in turn, are read as a sum of single-electron wavefunctions over two different sublattices:

$$
\begin{aligned}
& \phi_{1}=\sum_{A} e^{2 \pi i \mathbf{k} \cdot \mathbf{r}_{A}} X\left(\mathbf{r}-\mathbf{r}_{A}\right) \\
& \phi_{2}=\sum_{B} e^{2 \pi i \mathbf{k} \cdot \mathbf{r}_{B}} X\left(\mathbf{r}-\mathbf{r}_{B}\right)
\end{aligned}
$$

Here $\mathbf{r}_{A} \mathbf{r}_{B}$ are vectors pointing towards atoms from the corresponding sublattices, $X\left(\mathbf{r}-\mathbf{r}_{A}\right)$ and $X\left(\mathbf{r}-\mathbf{r}_{B}\right)$ - single electron wave functions, localized on these atoms. The secular equation (2.9) then reads:

$$
\left(\begin{array}{ll}
H_{11} & H_{12} \\
H_{21} & H_{22}
\end{array}\right)\left(\begin{array}{l}
c_{1} \\
c_{2}
\end{array}\right)=\left(\begin{array}{cc}
E S & E S_{12} \\
E S_{12} & E S
\end{array}\right)\left(\begin{array}{l}
c_{1} \\
c_{2}
\end{array}\right),
$$


where

$$
\begin{aligned}
H_{11} & =\int \phi_{1}^{*} H \phi_{1} d \mathbf{r} \\
H_{22} & =\int \phi_{2}^{*} H \phi_{2} d \mathbf{r} \\
H_{12} & =H_{21}^{*}=\int \phi_{1}^{*} H \phi_{2} d \mathbf{r} \\
S=S_{11}=S_{22} & =\int \phi_{1}^{*} \phi_{1} d \mathbf{r}=\int \phi_{2}^{*} \phi_{2} d \mathbf{r} \\
S_{12}=S_{21}^{*} & =\int \phi_{1}^{*} \phi_{2} d \mathbf{r}
\end{aligned}
$$

In the Tight-binding approximation (TBA), we neglect overlap of the wave functions of the neighboring atoms: $S_{12}=\int X\left(\mathbf{r}-\mathbf{r}_{A}\right) X\left(\mathbf{r}-\mathbf{r}_{B}\right) d \mathbf{r}=0$. Solving the secular equation (5.2), we obtain expression for energy:

$$
E=\frac{1}{2 S}\left(H_{11}+H_{22} \pm \sqrt{\left(H_{11}-H_{22}\right)^{2}+4\left|H_{12}\right|^{2}}\right)
$$

Physically there is no difference between the atoms belonging to the A and B sublattises, therefore $H_{11}=H_{22}$. Thus Eq. (5.3) simplifies to: $E=\frac{1}{S}\left(H_{11} \pm\left|H_{12}\right|\right)$. Substitution of Eq. (5.1) into $H_{12}$ yields

$$
H_{12}=\sum_{A, B} \exp \left[-2 \pi i \mathbf{k} \cdot\left(\mathbf{r}_{A}-\mathbf{r}_{B}\right)\right] \int X^{*}\left(\mathbf{r}-\mathbf{r}_{A}\right) H X\left(\mathbf{r}-\mathbf{r}_{B}\right) d \mathbf{r}
$$

The overlap matrix element $S=1$, since we assume the atomic wave functions to be normalized $\int X\left(\mathbf{r}-\mathbf{r}_{A}\right) X\left(\mathbf{r}-\mathbf{r}_{A}\right) d \mathbf{r}=1$. To find $H_{12}$ within the nearest-neighbor approximation, we simply sum over the three nearest neighbors shown in Fig. 5.1: $\mathbf{r}_{1}=\frac{a_{0}}{2}(1, \sqrt{3}), \mathbf{r}_{2}=\frac{a_{0}}{2}(1,-\sqrt{3}), \mathbf{r}_{3}=\frac{a_{0}}{2}(-2,1)$, then for the energy spectrum we 
obtain $^{1}$ :

$$
E_{ \pm}= \pm \gamma_{0} \sqrt{\left(1+4 \cos ^{2} k_{y} \frac{\sqrt{3}}{2} a_{0}+4 \cos k_{y} \frac{\sqrt{3}}{2} a_{0} \cos k_{x} \frac{3}{2} a_{0}\right)}
$$

where $\gamma_{0}=-\int X^{*}\left(\mathbf{r}-a_{0}\right) H X(\mathbf{r}) d \mathbf{r}$, where the plus sign gives the conductance band, and minus sign - valence band.

The Brillouin Zone of graphene forms a hexagonal prism, with basis vectors $\mathbf{b}_{\mathbf{1 , 2}}=$ $\frac{2 \pi}{3 a_{0}}(1, \pm \sqrt{3}, 0), \mathbf{b}_{\mathbf{3}}=\frac{2 \pi}{c_{0}}(0,0,1)$ (see Tab. 4.1). As the axial (c) lattice parameter grows, $b_{3} \rightarrow 0$ making the BZ essentially flat. Therefore we assume that the BZ under consideration is two-dimensional. The corners of the Brillouin Zone, called the Dirac points, have coordinates $K=\frac{2 \pi}{3 a_{0}}\left(1, \frac{1}{\sqrt{3}}\right), K^{\prime}=\frac{2 \pi}{3 a_{0}}\left(1,-\frac{1}{\sqrt{3}}\right)$, another point of interest lies in the middle of the BZ edge $M=\frac{2 \pi}{3 a_{0}}(1,0)$. The band structure within the TBA is plotted in Fig. 5.2. One can see that in the Dirac point $K E_{ \pm}=0$, i.e. pristine

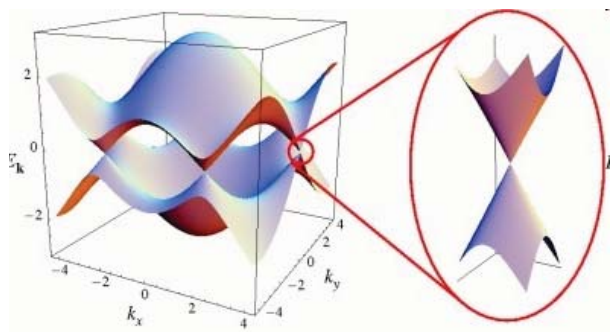

(a)

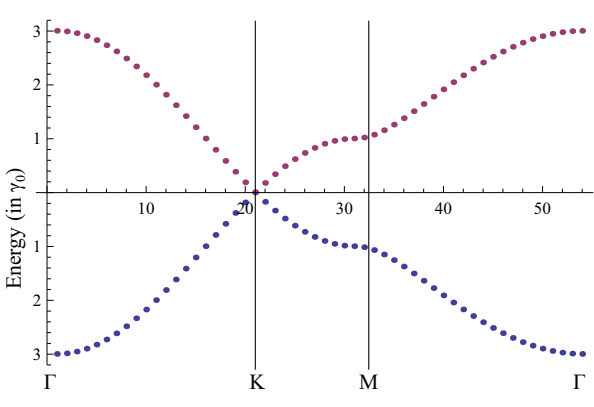

(b)

Figure 5.2: 3D representation (a) and a crossection through $\Gamma-K-M-\Gamma$ of graphene valence (blue) and conduction (red) bands

graphene has no band gap. Expanding the energy spectrum about the Dirac point, from Eq. (5.4) becomes $E_{K}\left(k_{x}, k_{y}\right)= \pm \frac{3}{2} a_{0} \gamma_{0} \sqrt{k_{x}^{2}+k_{y}^{2}}= \pm \frac{3}{2} a_{0} \gamma_{0}|\mathbf{k}|$. This dispersion relation is typical for massless fermions, and thus provides some insight on the nature of exceedingly high electron mobility in graphene, namely the reason for the ballistic

\footnotetext{
${ }^{1} H_{11}$ is not included into the calculation for it causes a constant energy shift
} 
transport mode in graphene. One can estimate the average speed of the carriers from the Dirac equation: $\hbar v_{F}=\frac{3}{2} a_{0} \gamma_{0}, a=1.42 \AA, \gamma_{0}=2.8 \mathrm{eV} \Longrightarrow v_{F} \approx 9 \times 10^{7} \mathrm{~cm} / \mathrm{s}$.

Most of the properties that drew wide attention to graphene exactly follow from this very fact that electrons in graphene behave like massless particles. This, however, causes a handicap - absence of the band gap. This makes graphene inapplicable for semiconductive application in microelectronics. However, from Eq. (5.3) it follows that if $H_{11} \neq H_{22}$, i.e. two carbon atoms in the unit cell are not equivalent (as it happens in graphite due to an offset of the next layer), an energy gap of magnitude $E_{g} \sim\left|H_{11}-H_{22}\right|$ occurs between the valence and the conductive bands. Therefore everything, that makes the atoms nonequivalent opens a band gap in graphene. Every functionalizing group disturbs in some way the electronic structure of the atom it is bound to, making the diagonal elements of the Hamiltonian matrix nonequivalent. Moreover, the stronger the disturbance of the local electronic structure, the wider is the gap. If functionalization occurs not to one of the three nearest neighbors, the TBA can be expanded by including more terms in the Hamiltonian matrix in Eq. (5.2). However, as long as at least one $H_{j j}$ coefficient is not equal to all other diagonal elements, a gap will be opened. Thus the value of the band gap will also change not only with the type of functionalizing molecule, but also with the ratio of the functionalized carbon atoms to the untouched ones. This model provides transparent physical background for functionalization of graphene.

Because of its simplicity the TBA is not suitable for calculation of complex many electron systems. Therefore we employed the Density Functional Theory (DFT) method to calculate the band structure of graphene. As it had already been discussed in Chapter II, the results extracted from the numerical solution of the Schrödinger equation strongly depend on the type of approximation used. A careless choice of the exchange-correlation functional, basis set, or the k-point sampling can lead to false 
results, or, colloquially speaking, "Garbage in - garbage out".

In the next two subsections the results obtained by means of the DFT formalism are presented.

\subsubsection{Convergence studies}

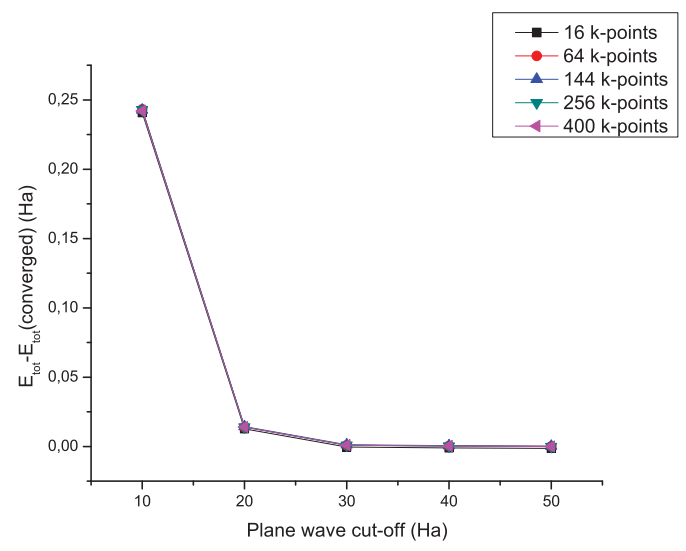

(a)

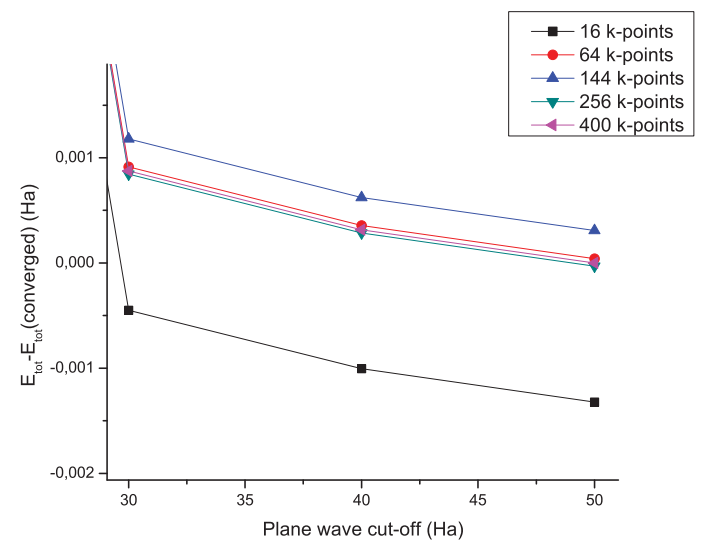

(b)

Figure 5.3: Convergence of graphene total energy with respect to the density of the k-point mesh

Convergence with respect to the number of k-points and the plane wave cutoff. Figure 5.3 depicts convergence studies with respect to both the k-point 
sampling grid and the energy cut-off value. It can be seen that increasing $E_{c u t}$ beyond $50 \mathrm{Ha}$ does not affect the total energy significantly. The system is also quite insensitive to the number of k-points in the IBZ: increase of it from 64 to 400 does not lower the total energy significantly. Therefore the 8x8x1 k-point grid yields equally precise results as the 20x20x1 at a much lesser computation cost.

Convergence with respect to the supercell size. As it was mentioned in section 2.5, undersampling of the $\mathrm{BZ}$ maximizes the unwanted interaction between the periodic images, therefore it is important to do convergence studies with respect to the size of the super cell chosen for the further calculations.

Axial supercell convergence. For graphene an important role is played by the spatial separation of the carbon layers: natural graphite has a so called AB stacking, in which every underlying layer is offset by $\frac{1}{2}(\vec{a}+\vec{b})$, where $\vec{a}$ and $\vec{b}$, are the lattice vectors (see Fig. 4.5). This nonequivalence between different carbon atoms, caused by the offset, creates two distinct types of atomic columns. This in turn according to Eq. (5.3) and the discussion in the end of section 5.1.1, disturbs the electronic structure of a single sheet. Therefore, it is important to realize at which separation graphitic layers stop "feeling" each other, i.e. the total energy of the system stops changing as the separation between the layers increases. Therefore, we calculated the total energy of graphite with different interlayer separation (see Table 5.1). As one can see form column $\Delta E_{t o t}$, the difference in total energies of graphite with interplanar separation of $5 c$ and $4 c$ is less than $2.8 \times 10^{-4} \mathrm{eV}$. The interlayer binding energy of graphite layers is computed as the difference between the total energies of graphite and graphene. Obtained result $\approx 50.5 \mathrm{meV}$ is in very good agreement with the experimentally observed value of $52 \pm 5 \mathrm{meV}$ [83]. Therefore, we assume that 
at the distance between graphene layers $>20 \AA$, the van-der-Waals interaction, that holds together the layers in graphite is entirely vanished. Based on that we have chosen $20 \AA$ to be $c$-lattice parameter in all our further calculations ${ }^{2}$.

\footnotetext{
${ }^{2}$ In this paragraph we limit our consideration by the supercell convergence studies relative to the interlayer separation, therefore each graphene sheet is assumed to be absolutely periodic constituted of small unit cells of dimensions $2.43 \times 2.43 \AA$ containing two carbon atoms per unit cell
} 


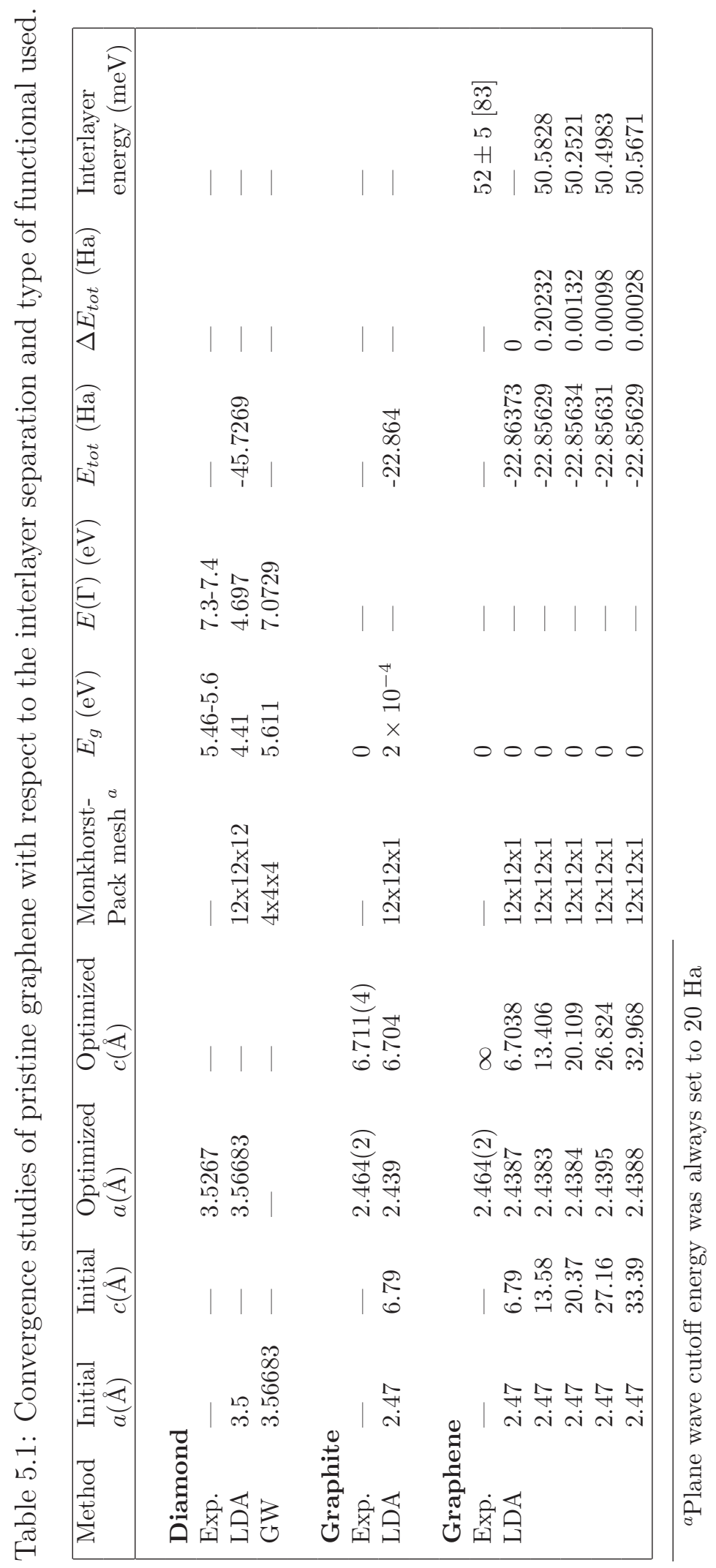


Lateral supercell convergence. As it was already mentioned in section 1.2, ideal 2D crystals are unstable due to diverging contribution of thermal fluctuations [11]. This discrepancy is resolved in graphene by local deplanation, also know as "buckling". Supercells of different lateral dimensions have a different ability to relax their ideal 2D structure and lower the total energy. We have performed geometry optimization and single point energy computations for $1 \times 1,2 \times 2,3 \times 3,4 \times 4,5 \times 5,6 \times 6$, 8x8 pristine graphene supercells.

Change in total energy per atom $\left(\Delta E_{t o t}\right)$ (see Fig. 5.4) as well as change in binding energy per atom $\left(\Delta E_{b i n d}\right)$ do not reveal any profound trend as the size of the super cell grows, as it might be expected if a significant relaxation of the atomic structure would have taken place.

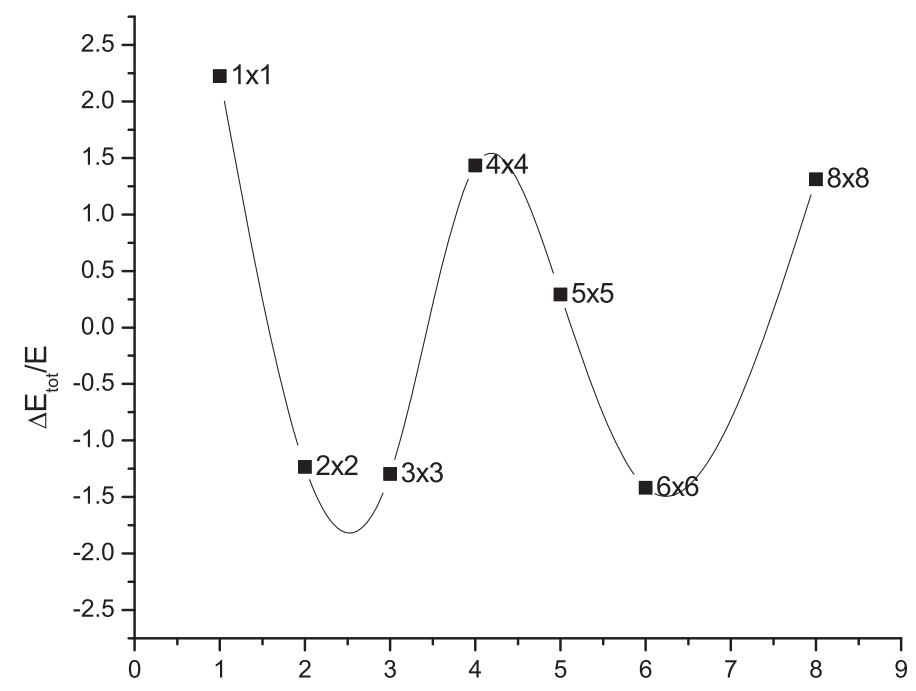

Figure 5.4: Relative variation of the total energy $\left(\frac{E_{t o t}-\left\langle E_{t o t}\right\rangle}{E_{t o t}}\right)$ in $\%$ as a function of the super cell size

Geometrical analysis of optimized super cells also showed no deplanation up to 8x8 suprecell. Therefore, hereafter we neglect the phenomenon of intrinsic deplanation, 
i.e. deplanation, caused solely by the Peierls instability of graphene. Deplanation caused by functionalization is a subject of careful examination, since it, in contrast to the intrinsic deplanation, is caused by rehybridization and thus local rearrangement of electronic structure.

\subsubsection{Band structure and Density of states (DoS)}

Energy of spectrum of pristine graphene has been an object of great interest

for last five years. During this time it has been calculated multiple times using different ab-initio and semi-empirical methods, as well as different approximations within the given method $[84,85,86,87,88,89]$. Here current calculations are present for benchmarking purposes. Our calculations reveal very close coincidence with the results published before, in e.g. [84]. The spectrum in Fig. 5.5, calculated by means of the DFT reveals a close similarity to the spectrum computed using the TBA (Fig.

The density of states possesses a typical peculiarity - absence of states at the Fermi level. This peculiarity comes from the linear dispersion spectrum in the vicinity of the Dirac point $(\mathrm{K})$, and contrasts to the quadratic energy-momentum relation obeyed by electrons at band edges in conventional semiconductors.

\subsubsection{Electron density and wave functions}

The wavefunction manifold of graphene consists of a group of binding $\sigma$ - and $\pi$ orbitals and non binding unhybridized $p_{z}$ orbitals. The $\sigma$ orbitals are created by the 


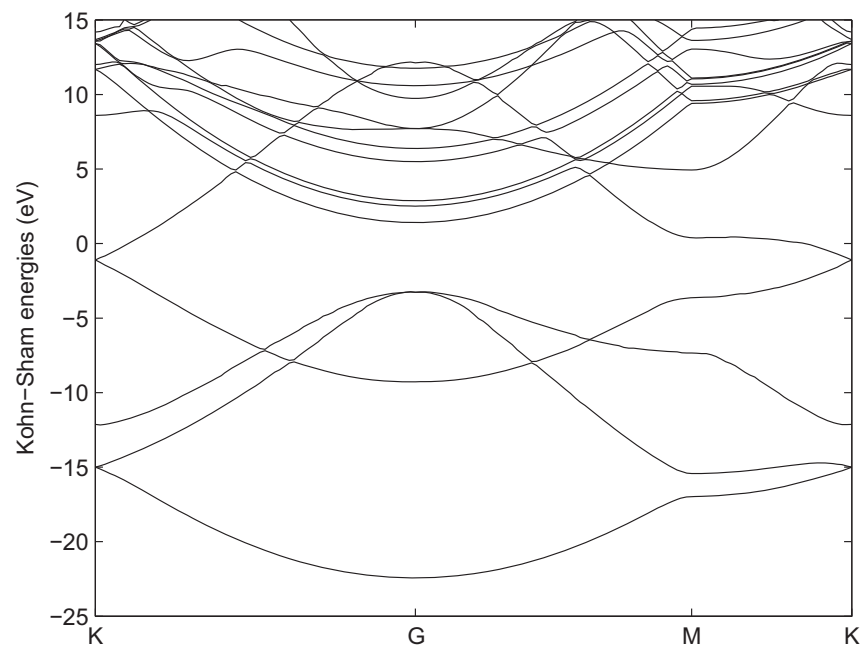

(a)

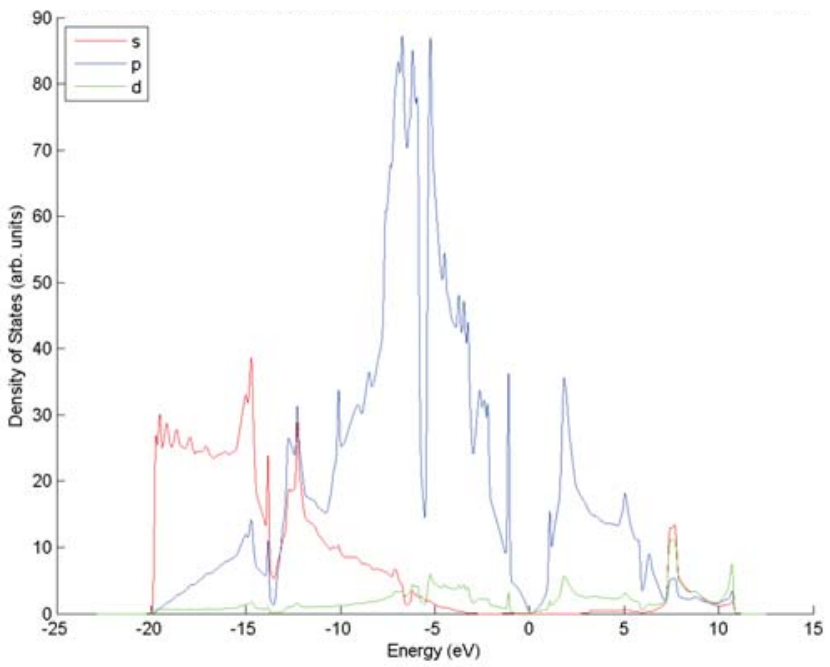

(b)

Figure 5.5: Band structure (a) and Density of states (DoS) (b) calculated from the DFT methods. 
$s p^{2}$ hybridization process:

$$
\begin{aligned}
\phi_{1}^{A, B} & =\frac{1}{\sqrt{3}} s^{A, B} \pm \sqrt{\frac{2}{3}} p_{x}^{A, B} \\
\phi_{2}^{A, B} & =\frac{1}{\sqrt{3}} s^{A, B} \mp \frac{1}{\sqrt{6}} p_{x}^{A, B} \pm \frac{1}{\sqrt{2}} p_{y}^{A, B} \\
\phi_{3}^{A, B} & =\frac{1}{\sqrt{3}} s^{A, B} \pm \frac{1}{\sqrt{6}} p_{x}^{A, B} \pm \frac{1}{\sqrt{2}} p_{y}^{A, B} \\
\phi_{4}^{A, B} & =p_{z}^{A, B}
\end{aligned}
$$

From the diagram in Fig. 5.6 it follows that electrons in graphene occupy three

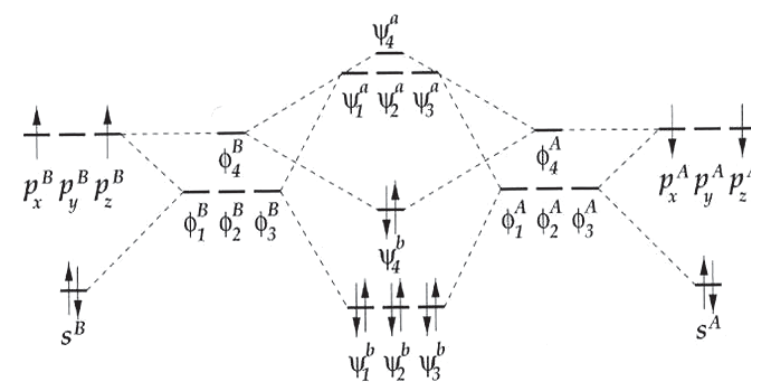

Figure 5.6: Molecular orbital diagram of graphene

binging states and one non binding. The symmetry of the binding states reads as $C_{3 h}$ and the symmetry of the non binding state $-D_{\infty v}$. The wavefunctions are obtained as a sum of the Bloch states over the full BZ (or IBZ with respect to the symmetry multiplication):

$$
\Psi_{i}(\mathbf{r})=\frac{1}{\Omega_{B Z}} \int_{B Z} \psi_{i \mathbf{k}} d \mathbf{k}=\frac{1}{\sqrt{N}} \sum_{\mathbf{k} \in B Z} \psi_{i \mathbf{k}} w_{\mathbf{k}}
$$

However, if waverfunctions for the first four zones are plotted, they do not possess the necessary symmetry. The reason for that is the so called "entanglement": conventional DFT algorithms are k-point independent, i.e. the coordinates of every k-point are fixed parameters in the Schrödinger equation. Thus the solution of the Schrödinger equation for each k-point is a discreet set of numbers - the Kohn-Sham eigenvalues of 
the system (corresponding to the energy levels of the system). Then these numbers are ordered in the ascending order, without regard to the bands symmetry. Therefore, when two bands intersect, they "flip" due to the ordering of the eigenvalues. Therefore in Eq. (5.6) the numbers of the wavefunctions $(i)$ are randomly permuted (entangled). In order to avoid this a disentanglement procedure (described in Sec 3.3) is necessary.

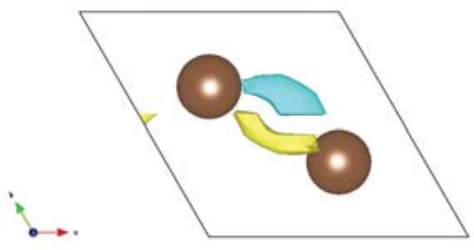

(a) Band \#1
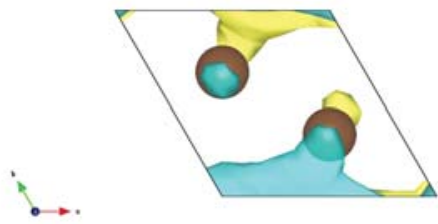

(c) Band \#3

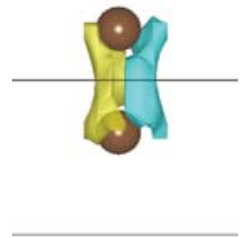

(b) Band \#2

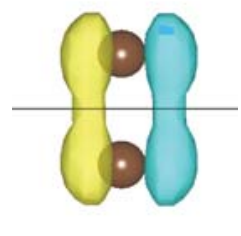

(d) Band \#4

Figure 5.7: Wavefunctions corresponding to the first four bands in graphene

The result of such disentanglement is a continuous (with respect to the k-vector) band structure. Disentanglement is done concurrently with the Wannierization procedure. Therefore, Wannier functions possess the necessary symmetry property ${ }^{3}$. The electron density is computed as $\rho(\mathbf{r})=\sum_{\mathbf{k} \in B Z} \sum_{i \in o c c}\left|\psi_{i \mathbf{k}}(\mathbf{r})\right|^{2}=\sum_{i \in o c c} \rho_{i}(\mathbf{r})$ and because of a summation over all valence bands $(i)$, electron density is not affected by entanglement. As it is expected, electrons are primarily localized between carbon atoms in the plane of the sheet, which coincides with earlier experimental data [90].

\footnotetext{
${ }^{3}$ Wannier functions can be considered as the same type of summation as in Eq. (5.6), in which the weights $\left(w_{\mathbf{k}}\right)$ are chosen to be $e^{-i \mathbf{k} \cdot \mathbf{R}_{\mathbf{n}}}$
} 


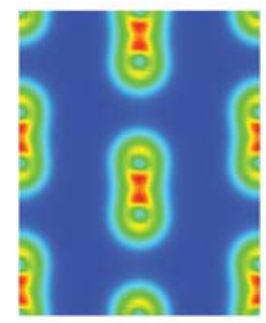

(a)

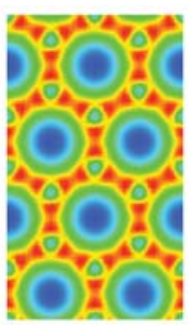

(b)

Figure 5.8: Valence electron density in graphene. (a) [100] orientation (the plane of atoms) (b) [001] orientation (the plane perpendicular to atoms)

\subsubsection{Wannier functions in pristine graphene.}

The Wannierization procedure starts with a definition of a trial set of Wannier functions $^{4}$. From the nature of bonding, represented by the MO diagram in Fig. 5.6 it follows that a sufficient set of trial Wannier functions should include three $\sigma$ type functions (rendered as $\left.\phi_{1,2,3}\right)$ and one $p_{z}$ type function $\left(\phi_{4}\right)$. Since there are two carbon atoms per graphene supercell, two $p_{z}$ trial functions are necessary. 20 bands were considered, from which 5 Wannier functions were constructed in the energy range of the "frozen-states" window, which was chosen to be from $E_{F}+2.0 \mathrm{eV}$, to $-\infty$. Disentanglement converged after 27 iterations, Wannerization converged after 441 iterations giving the total spread operator decomposition (in Bohr ${ }^{2}$ ):

$$
\begin{aligned}
\Omega_{I} & =10.101209 \\
\Omega_{D} & =0.040114 \\
\Omega_{O D} & =2.635888 \\
\Omega_{T o t} & =12.777211
\end{aligned}
$$

From the bond orbitals (essentially Wannier functions) of $\sigma$ type $\left(\phi_{1,2,3}\right.$, Fig. 5.9a)

\footnotetext{
${ }^{4} \mathrm{~A}$ random set can be used as a trial set, but it requires many more iterations and perfectly follows the principle "garbage in - garbage out"
} 


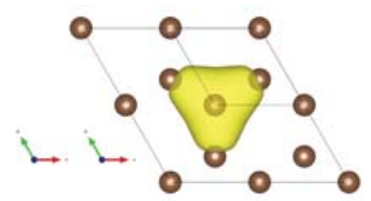

(a) $s$-orbital

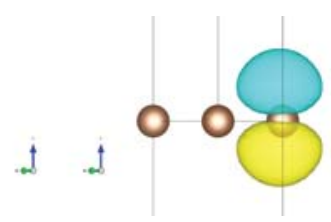

(d) $p_{z}$-orbital

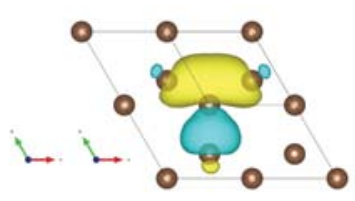

(b) $p_{x}$-orbital

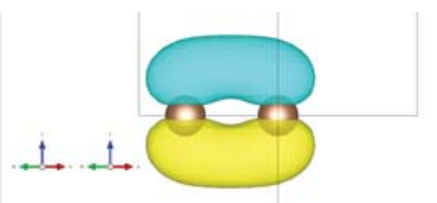

(e) $\pi$-orbital

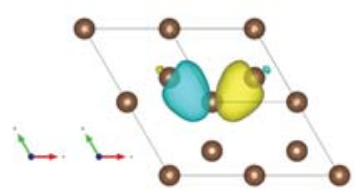

(c) $p_{y}$-orbital

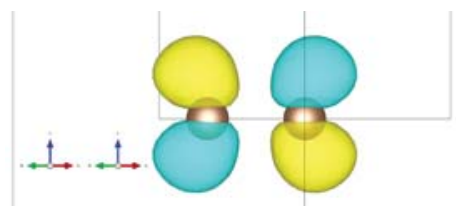

(f) $\pi^{*}$-orbital

Figure 5.9: Wannier functions in graphene.

we can reconstruct the actual $p_{x}$ (Fig. 5.9b) and $p_{y}$ (Fig. 5.9c) atomic like orbitals as: $p_{x}=\phi_{1}+\phi_{2}-\phi_{3}$ and $p_{y}=\phi_{1}-\phi_{2}-\phi_{3}$. (See Eq. (5.5)) From the $p_{z}$ Wannier function we reconstruct bonding (Fig. 5.9e) and antibonding (Fig. 5.9f) $\pi$-orbitals.

The set of Wannier functions in Fig. 5.9 constitutes a complete manifold of wavefunctions and can be used to interpolate the band structure as described in section 3.4

\subsubsection{Disentangled bands and ballistic quantum transport in graphene.}

From the disentanglement procedure we obtain an interpolated band structure that contains as many bands as the number of Wannier functions. This band structure does not suffer from arbitrary arrangement of eigenvalues of the Hamiltonian matrix. Therefore, comparing Fig. 5.5 with Fig. 5.10 we see that the top of the $\pi^{*}$ band is disentangled from the upper set of conductive bands even though they have lower energy at the $\Gamma$ point than the $\pi^{*}$ band.

Ballistic transport through graphene sheet is calculated in accordance with the formalism outlined in chapter III. All five Wannier functions were used to construct a localized representation of the Hamiltonian matrix; $400 \mathrm{k}$-points in the direction of transport were used to reconstruct the band structure. 


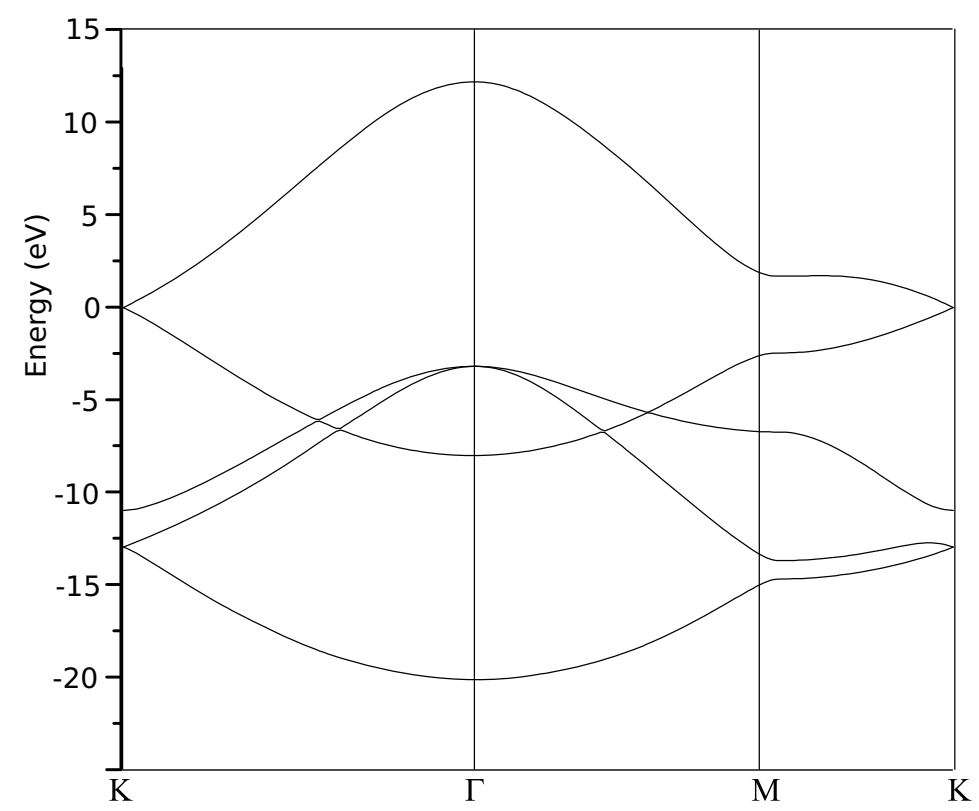

Figure 5.10: Disentangled WF interpolated band structure of graphene.

In the $\Gamma$-M direction (Fig. 5.12), graphene behaves like a typical semiconductor with the band gap of about $5 \mathrm{eV}$. Abrupt edges of the conductivity curve arise from the van Howe singularities in the DoS spectrum (panel c): as the bands flatten out, the density of states and electron's effective mass approach infinity, thus the mobility vanishes, and hence drop in the conductance.

In the $\Gamma$-K direction (Fig. 5.11), however, graphene demonstrates its unique properties it has become to be known for - the Dirac cones and the electron dispersion spectrum linear in the vicinity of the Dirac point. The density of states is zero only at the Fermi energy, decaying also linearly in the vicinity of that point. The conductance curve pretty much mimics the density of states, about the neutrality (Dirac) point, and further deviates from it as van Howe singularities start to appear in the band structure.

From the Landauer theory it follows that in case of a pure ballistic transport, conductivity is proportional to the number transport modes, i.e. the number of bands 

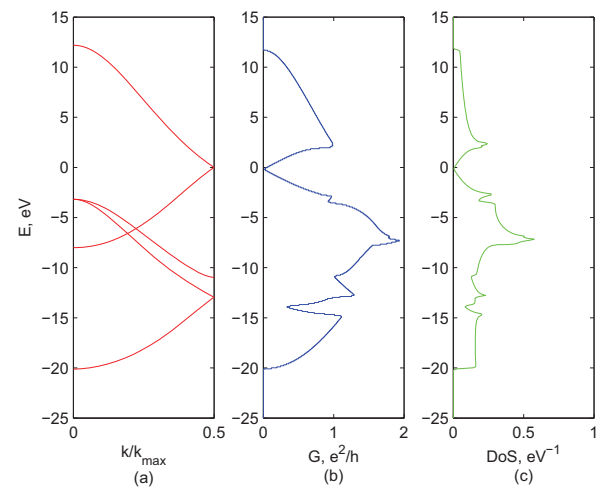

Figure 5.11: Band structure (a), ballistic transmission function (b), and density of states (c) of graphene in the $\Gamma$-K direction.
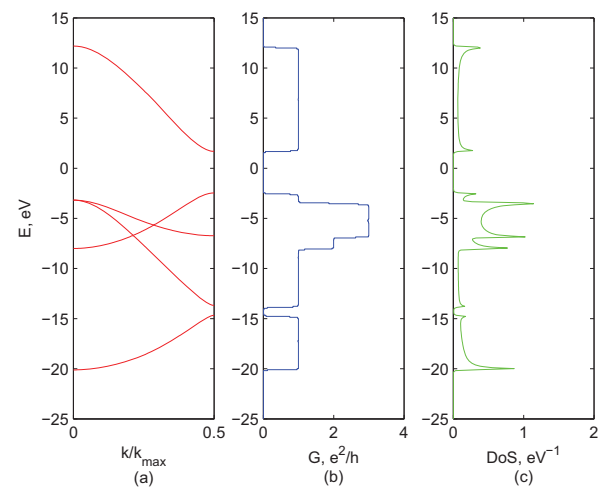

Figure 5.12: Band structure (a), ballistic transmission function (b), and density of states (c) of graphene in the $\Gamma-\mathrm{M}$ direction.

crossed an any energy ordinate. This is true in case of a semiconductor, where the Bloch states can be separated into two subsets - a finite set of valence bands and an infinite one of the conduction bands (See section 3.3.1). In a metal or a semimetal (as graphene) those two sets intersect, which allows the easy hopping of electrons between the conduction bands and the valence bands. Due to the absence of band gap, the curvature of the bands at the BZ boundary does not need to be zero, therefore no van Howe singularities are present in graphene around the charge neutrlaity point. Therefore, the DoS does not possess any sharp peaks, corresponding to electron's infinite effective mass, therefore each mode does not have an abrupt breakdown. 
This all leads to the difference between "ballistic" transmission function (which is just a sum over all eigenchannels) and the conductivity calculated using the Green's functions formalism (Fig. 5.13).

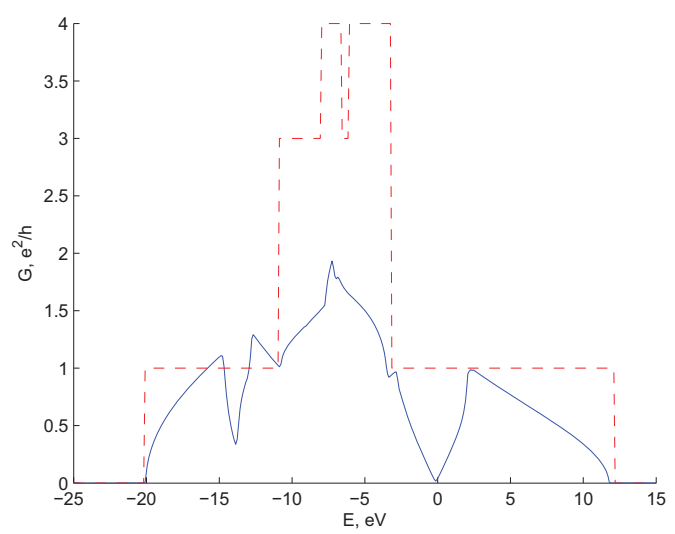

Figure 5.13: Comparison between the number of eigenmodes (dashed) and the transmission function (solid) in the $\Gamma-\mathrm{K}$ direction in graphene.

In figure 5.13 one can see that the minima and the maxima of the conduction curve correspond to the energies at which the corresponding eigenmode gets excited. The value of the transmission function at these energies (at the $\mathbf{k}$-points corresponding to these energies) is proportional to the coupling between the corresponding Bloch (or Wannier) functions describing intersecting modes: $\left\langle\psi_{\mathbf{k} n} \mid \psi_{\mathbf{k} m}\right\rangle$.

I-V curves can be obtained by integration of the conductivity curves, using Eq. (3.14), in this case $\mu_{R}=\mu_{R}+e V$, where $\mathrm{V}$ is the bias voltage. Resulting I-V curves are depicted in Fig. 5.14.

One can see that the slope of the $\mathrm{I}-\mathrm{V}$ curve in the $\Gamma-\mathrm{K}$ direction is never zero, unlike that the curve for the $\Gamma-\mathrm{M}$ direction has a well-pronounced plateau around the neutrality (zero bias) point. Even though those phenomena had been studied before both theoretically [91, 92] and experimentally [79] they are still relevant to be presented here for the didactic purpose as well as for the convergence studies 

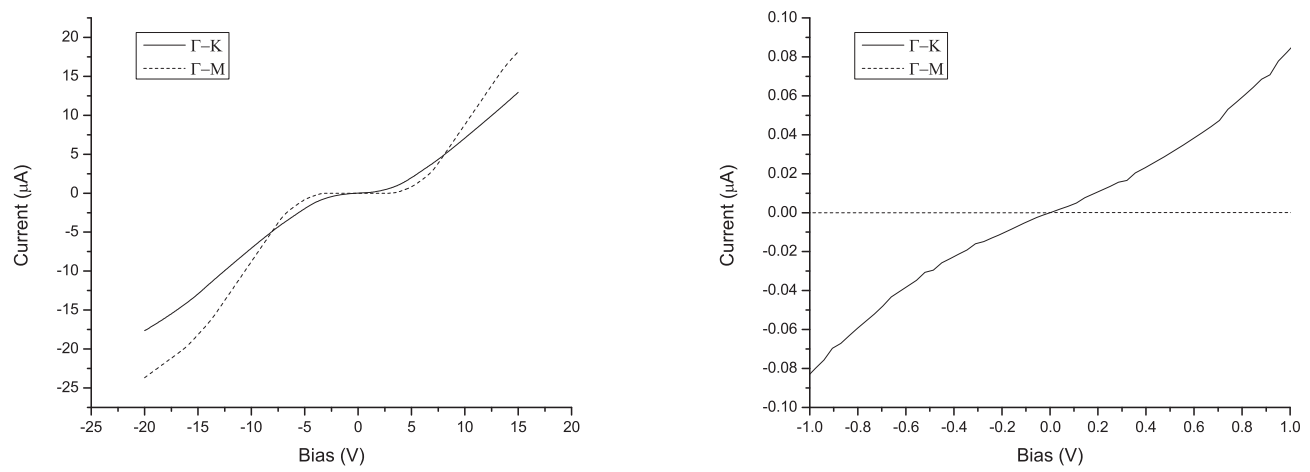

Figure 5.14: I-V curves of graphene. In the inset: zoomed I-V curve in the the vicinity of zero bias

important for the future computations. Usually disentanglement and wannierization require quite a sparse k-point mesh. As recommended in [52], it can be kept below 10 k-points along the longest BZ dimension. However, due to the interpolative properties of Wannier functions, after wannierization we can operate on much denser k-points grids for band structure interpolation and transport computation. In Fig. 5.15 the conductance curves are presented depending on the number of k-points in the direction of conductance:

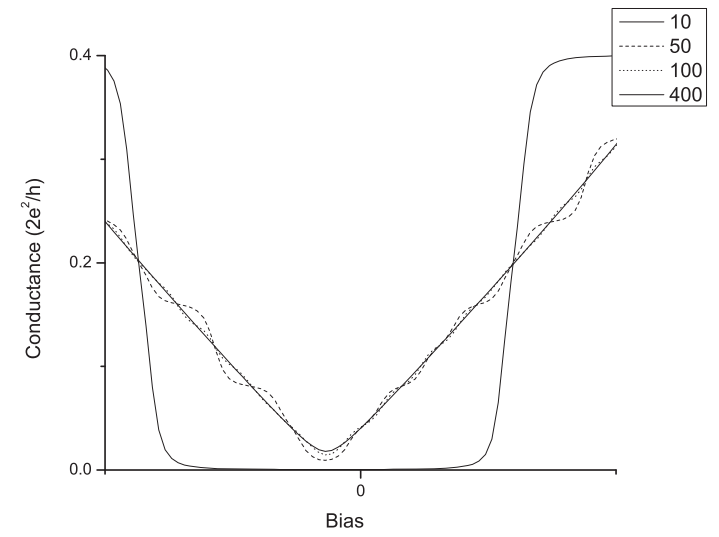

Figure 5.15: Conductance curves for 10, 50, 100, and 400 k-points along the direction of conductance 
From figure 5.15one can see that only $100 \mathrm{k}$-points (and above) are sufficient for proper interpolation of the conductance, even despite the fact that only $36 \mathrm{k}$-points were used to obtain the Bloch wave representation of the wave function. Therefore for all further conductance calculations are performed on denser k-point grids. The density of the grid depends on the size of the supercell and should be correspondingly reduced as the size of a supercell increases in the direct space.

\subsection{Atomic and electronic structure of GNR}

Graphene nanoribbons can be produced from an infinite graphene sheet by "controlled nanocutting" of graphene [93]. Careful intelligent tailoring of graphene edges opens a way towards a finer control of physical properties of graphene. Theoretical calculations indicate that, depending on the size, geometry, and edge structure, GNRs should display a variety of electronic properties. Special attention is paid to GNRs due to dependence of their electronic properties on the type of the boundary (chirality).

\subsubsection{Atomic structure and chirality of GNRs}

Atomistic structure of a GNR can be understood in terms of the chiral angle $\phi$ and the width $L$. The chiral angle is related to the corresponding CNT indices, (n,m), and represents the crystallographic direction of the axis of the GNR. The term chirality come from theoretical studies of CNTs, where the nanotube is actually twisted or winding around its axis. Since atomistic structure of GNRs considered here, are obtained by "unfolding" of infinite periodic CNTs and cutting (or extending) the resulting sheet to the desired width, it is natural to adopt the same terminology for the GNRs, even if the term "chirality" had lost its original meaning. A set of 
parameters listed in table 5.2 is important for consideration of the atomic structure of the the GNRs. Among all the possible chiral angles (or CNT indices) special attention is payed to the "zigzag" $(n, n)$ and "armchair" $(n, 0)$ nanoribbons (see Fig. 5.16 and table 5.3).

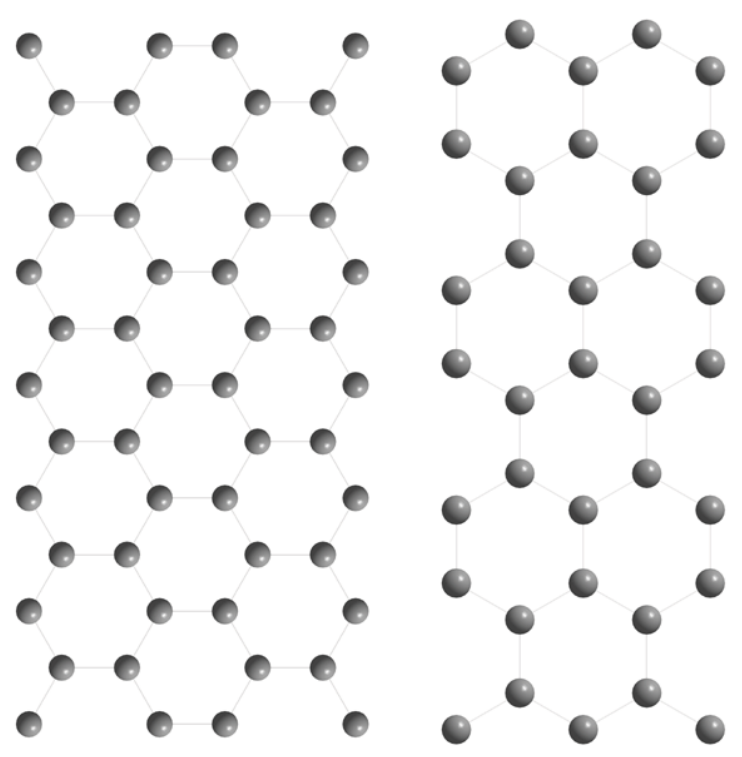

(a)

(b)

Figure 5.16: Zigzag (a) and armchair (b) GNRs

Zigzag GNRs are always metallic (except for very narrow GNRs, which are semiconducting with the band gap inversely proportional to the GNR width $[79,94]$ ), while armchair GNRs are either metallic or semiconducting, depending on the GNR width [19]. It has been demonstrated that a combination of two ribbons of different chirality in the same device can lead to a Schottky type contact and thus create a heterojunction [95]. Zigzag GNRs can be obtained form an infinite graphene sheet by cutting in the (100) direction, whereas armchair - in the (110) direction (see Fig. $5.17)$.

In the reciprocal space those directions correspond to the $\Gamma-\mathrm{M}$ and $\Gamma-\mathrm{K}$ paths 


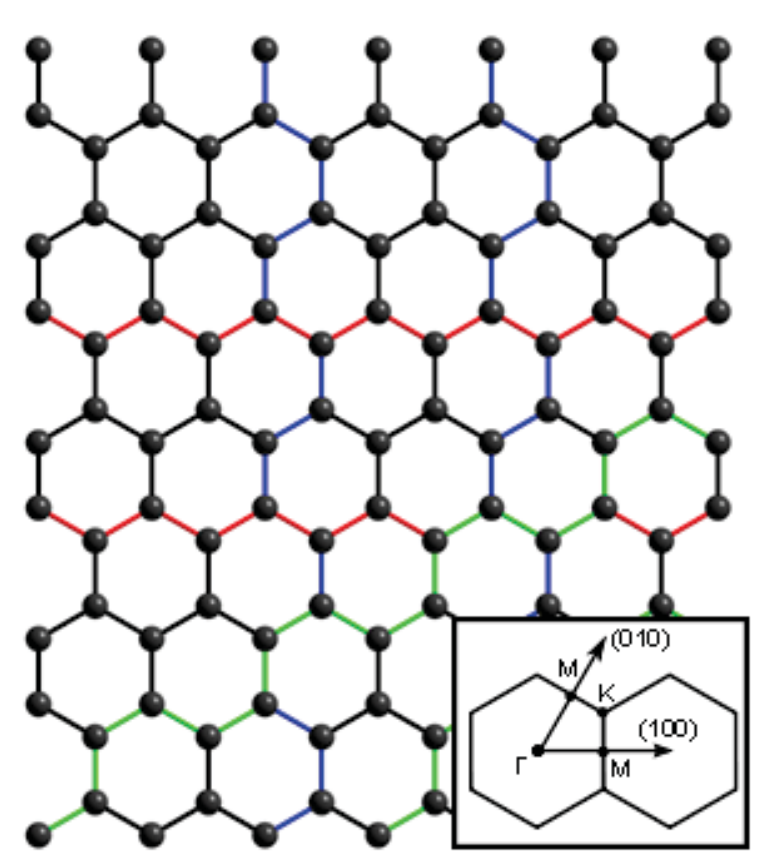

Figure 5.17: Cuts along two directions of a graphene sheet, to produce zigzag (red) and armchair (green, blue) terminations of the GNRs

in the BZ for zigzag and armchair terminations, respectively. For convenience, the atomic structure of infinite graphene sheet was converted into the orthogonal setting using the transformation matrix from Eq. (4.6). After this transformation $\Gamma$ - M, i.e. the "zigzag path" maps onto the $\Gamma$ - X direction and $\Gamma$ - K, i.e. the "armchair path", onto the $\Gamma$ - Y direction. There are two different directions in the graphene structure that have the same boundary structure: blue and green lines in Fig. 5.17. Since the structure is periodic only in one direction, the BZ path for all dispersion calculation was chosen as $\Gamma-\Sigma-\mathrm{X}$.

\subsubsection{Band structure of GNR}

The electronic structure of a GNR can be obtained simply from that of infinite graphene. The wave vector in the lateral direction becomes quantized, whereas the axial vector remains continuous for a GNR of infinite length. Thus the energy bands 
Table 5.2: Geometric parameters of graphene and GNR atomic structure.

\begin{tabular}{|c|c|c|}
\hline Symbol & Name & Formula \& Value \\
\hline$a$ & length of the unit vector & $a=\sqrt{3} a_{C-C}=2.49 \AA, a_{C-C}=1.44 \AA$ \\
\hline $\mathbf{a}_{1}, \mathbf{a}_{2}$ & unit vectors & $\left(\frac{\sqrt{3}}{2}, \frac{1}{2}\right) a,\left(\frac{\sqrt{3}}{2},-\frac{1}{2}\right) a$ \\
\hline$b_{1}, b_{2}$ & reciprocal unit vectors & $\left(\frac{1}{\sqrt{3}}, 1\right) \frac{2 \pi}{a},\left(\frac{1}{\sqrt{3}},-1\right) \frac{2 \pi}{a}$ \\
\hline$d$ & $\operatorname{gcd}(n, m)$ & \\
\hline$d_{R}$ & $g c d(2 n+m, 2 m+n)$ & $d_{R}= \begin{cases}d, & \text { if }(n-m) \text { is multiple of } 3 \\
3 d, & \text { if }(n-m) \text { is not a multiple of } 3\end{cases}$ \\
\hline $\mathbf{L}$ & $\begin{array}{l}\text { unit vector in the lateral di- } \\
\text { rection (chiral indices) }\end{array}$ & $\mathbf{L}=n \mathbf{a}_{\mathbf{1}}+m \mathbf{a}_{\mathbf{2}}=(n, m)$ \\
\hline$L$ & width of a GNR & $L=|\mathbf{L}|=a \sqrt{n^{2}+m^{2}+n m}$ \\
\hline $\mathbf{T}$ & axial translation vector & $\begin{array}{l}\mathbf{T}=t_{1} \mathbf{a}_{1}+t_{2} \mathbf{a}_{2}=\left(t_{1}, t_{2}\right) \\
t_{1}=\frac{2 m+n}{d_{R}}, t_{2}=-\frac{2 n+m}{d_{R}}\end{array}$ \\
\hline$T$ & $\begin{array}{l}\text { unit cell length in the axial di- } \\
\text { rection }\end{array}$ & $T=|\mathbf{T}|=\frac{\sqrt{3} L}{d_{R}}$ \\
\hline$\phi$ & chiral angle & $\tan \phi=\frac{\sqrt{3} m}{2 n+m}$ \\
\hline$N$ & $\begin{array}{l}\text { number of honeycombs in the } \\
\text { lateral direction }\end{array}$ & $N=\frac{2\left(n^{2}+m^{2}+n m\right)}{d_{R}}$ \\
\hline $\mathbf{K}_{1}, \mathbf{K}_{2}$ & $\begin{array}{l}\text { reciprocal lattice vectors of a } \\
\text { GNR }\end{array}$ & $\begin{aligned} \mathbf{K}_{\mathbf{1}} & =\frac{1}{N}\left(-t_{2} \mathbf{b}_{\mathbf{1}}-t_{1} \mathbf{b}_{\mathbf{2}}\right) \\
\mathbf{K}_{\mathbf{2}} & =\frac{1}{N}\left(-m \mathbf{b}_{\mathbf{1}}-n \mathbf{b}_{\mathbf{2}}\right)\end{aligned}$ \\
\hline
\end{tabular}

Table 5.3: Geometric parameters for zigzag and armchair terminations

\begin{tabular}{lllllllll}
\hline Chirality & $\mathbf{L}$ & $L / a$ & $d$ & $d_{R}$ & $\mathbf{T}$ & $T / a$ & $N$ & $\mathbf{K}_{\mathbf{1}}, \mathbf{K}_{\mathbf{2}}$ \\
\hline Zigzag & $(n, n)$ & $\sqrt{3} n$ & $n$ & $3 n$ & $(1,-1)$ & 1 & $2 n$ & $\begin{array}{l}\mathbf{K}_{\mathbf{1}}=\frac{1}{2 n}\left(2 \mathbf{b}_{\mathbf{1}}+\mathbf{b}_{\mathbf{2}}\right), \\
\end{array}$ \\
& & & & & & & $\mathbf{K}_{\mathbf{2}}=-\frac{1}{2} \mathbf{b}_{\mathbf{2}}$ \\
Armchair & $(n, 0)$ & $n$ & $n$ & $n$ & $(1,-2)$ & $\sqrt{3}$ & $2 n$ & $\begin{array}{l}\mathbf{K}_{1}=\frac{1}{2 n}\left(\mathbf{b}_{\mathbf{1}}+\mathbf{b}_{\mathbf{2}}\right), \\
\mathbf{K}_{\mathbf{2}}=\frac{1}{2}\left(\mathbf{b}_{\mathbf{1}}-\mathbf{b}_{\mathbf{2}}\right)\end{array}$ \\
\hline
\end{tabular}


consist of a set of one-dimensional energy dispersion relations which are cross sections of those for infinite graphene (see Fig. 5.2a).
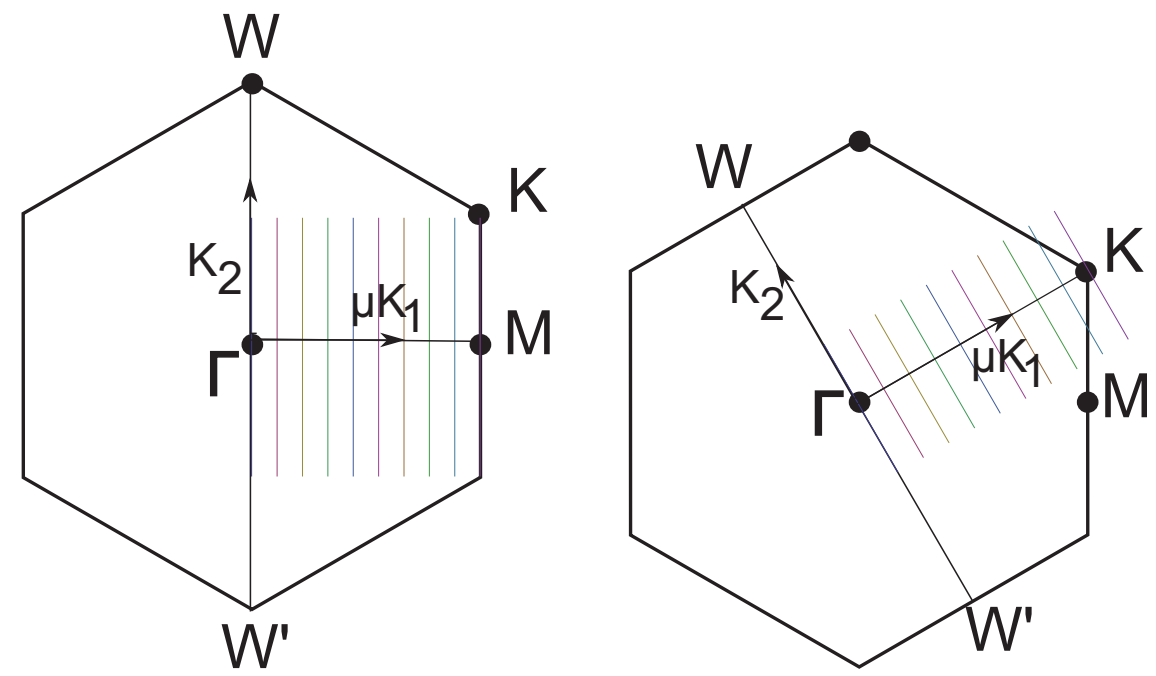

Figure 5.18: Folding of 2D graphene band structure for armchair (left) and zigzag (right) terminations.

When the energy dispersion relations of two-dimensional graphite, $E_{g 2 D}(k)$ (see Eq. (5.4)) at line segments are shifted from WW' by $\mu \mathbf{K}_{\mathbf{1}}$ vector $(\mu=0, \ldots, N-1)$ are overlayed with each other so that the reciprocal vectors parallel to $\mathbf{K}_{\mathbf{2}}$ coincide with WW' direction as shown in Fig. 5.18, N pairs of 1D energy dispersion curves $E_{\mu}(k)$ are obtained. These $1 \mathrm{D}$ energy dispersion relations are given by:

$$
E_{\mu}^{G N R}(k)=E_{g 2 D}\left(k \frac{\mathbf{K}_{\mathbf{2}}}{\left|\mathbf{K}_{\mathbf{2}}\right|}+\mu \mathbf{K}_{\mathbf{1}}\right)
$$

where $\mu=0, \ldots, N-1$, and $-\frac{\pi}{T}<k<\frac{\pi}{T} . N, T, \mathbf{K}_{\mathbf{1}}$, and $\mathbf{K}_{\mathbf{2}}$ (the reciprocal lattice vectors of the a GNR) are defined in Tab. 5.2.

The N pairs of energy dispersion curves given by Eq. (5.7) correspond to the cross sections of the two-dimensional energy dispersion surface shown in Fig. 5.2a, where cuts are made along the lines defined by the equation: $k \frac{\mathbf{K}_{\mathbf{2}}}{|\mathbf{K} \mathbf{2}|}+\mu \mathbf{K}_{1}=0$. This type of folding can be considered as the ultimate case of folding described in Sec. 2.6, for a 
supercell $\infty$ x1x1, when each high order BZ is degenerated into a line. If the cutting line passes through the Dirac point of the 2D Brillouin zone, where the conduction and the valence energy bands of pristine graphene touch each other, the one-dimensional energy spectra have a zero energy gap, and thus the GNRs are metallic ${ }^{5}$.

Since any real nanodevice is essentially some sort of a GNR, the knowledge of the 3D band structure of graphene whether pristine or functionalized, provides an algorithm to deduce electron dispersion relations in such a nanodevice. Ultimately, in the limit of very broad GNRs, $\mathbf{K}_{\mathbf{1}} \rightarrow 0$, the GNR band structure becomes that of graphene for $\Gamma-K$ or $\Gamma-M$ path.

\subsection{Electric conductance of graphene and GNRs}

Preliminary SCF and nonSCF calculations were done using the QUANTUM-Espresso program suite [66], the computation of the conductance with the NEGF approach as described in Chap. III were performed using the WANT program suit [52].

A detailed discussion of the choice of the parameters for the computation will be given here on an example of a 2-ZGNR (Fig. 5.19).

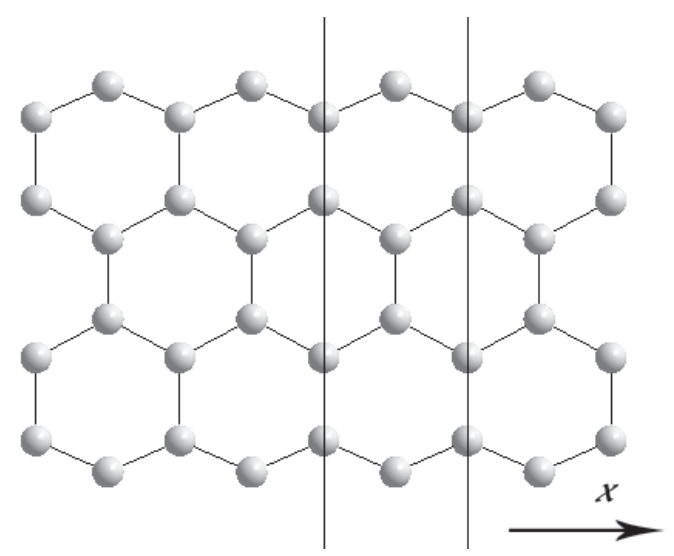

Figure 5.19: Strucutre of a 2zGNR.

\footnotetext{
${ }^{5}$ However, due to Peierls instability, a small gap opens up even in metallic GNRs, especially in very narrow ones. See e.g. [96]
} 
Cell dimensions should be optimized first to exclude unwanted interaction between periodic images of the same GNR. The size of the unit cell used in the computation was $a=2.5739 \AA, b=71,4795 \AA, c=20 \AA$. The unit cell size dictates the density of the k-points. Since we are interested in 1D-transport, electron dispersion in lateral direction can be ignored. The direction of translational periodicity is chosen to be $x$. Therefore the k-point mesh is chosen to be the non-shifted 3x1x1 Monkhorst-Pack grid. The structure contains eight carbon atoms in the unit cell, 32 valence electrons, and 16 core electrons. These electrons fully occupy 24 bands. The rule of thumb for good convergence suggests that the number of empty bands to consider should at least three times the number of occupied ones, which gives a total of 72 bands to consider.

\subsubsection{Wannier function calculation}

The number of Wannier functions to consider was chosen from the following considerations: one $\sigma$-type trial function with a Gaussian radial part, localized in the middle of the bond, and one $p_{z}$-type trial function with a Laguerre radial part, lo-

calized on each carbon atom. Some of them resemble WFs initialized for the infinite graphene sheet, i.e. belong to the periodic manifold Others, however, arise from the dangling bonds at the GNR edges. A total of 20 trial Wannier functions were initialized: 12 Gaussians and 8 Laguerres. All 72 bands were considered, top of the "frozen-states" window was chosen to be $E_{F}+2.0 \mathrm{eV}$, an the bottom was $-\infty$. Disentanglement procedure converged after 24 steps. Localization procedure converged 
after 1327 iterations, giving the spread operator decomposition (in $\mathrm{Bohr}^{2}$ ):

$$
\begin{gathered}
\Omega_{I}=40.564428 \\
\Omega_{D}=139.400272 \\
\Omega_{O D}=21.292256 \\
\Omega_{T o t}=201.256956
\end{gathered}
$$

Localized Wannier functions are also tested visually: they should be symmetric, centered in proper positions, form a closed isosurface with no "podia", and be real-valued. The Wannier functions in bulk graphene are represented by the $\sigma$-type WF, and $p_{z}$ WF, which in the MO picture correspond to hybridized $\sigma$-orbitals (Fig. 5.20a) and unhybridized $p_{z}$ (Fig. 5.20b). Those are also found in GNRs (Fig. 5.20).

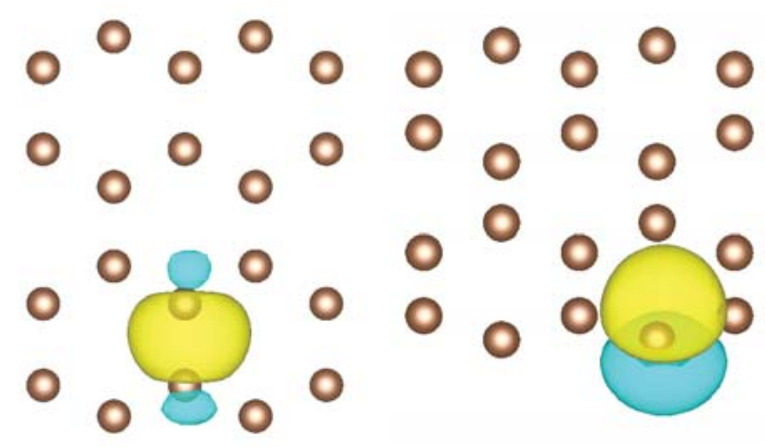

(a) $\sigma$-type WF $p_{z}$-type WF (side
view)

Figure 5.20: Wannier functions in graphene from the periodic manifold

Also since GNR lacks periodicity in the lateral direction, some extra states are found in GNRs, which are absent in infinite graphene (Fig. 5.21), like dangling $\sigma$-bonds (Fig. 5.21a), and binding $\pi$-orbitals (Figs. 5.21b, 5.21c) representing edge states. 


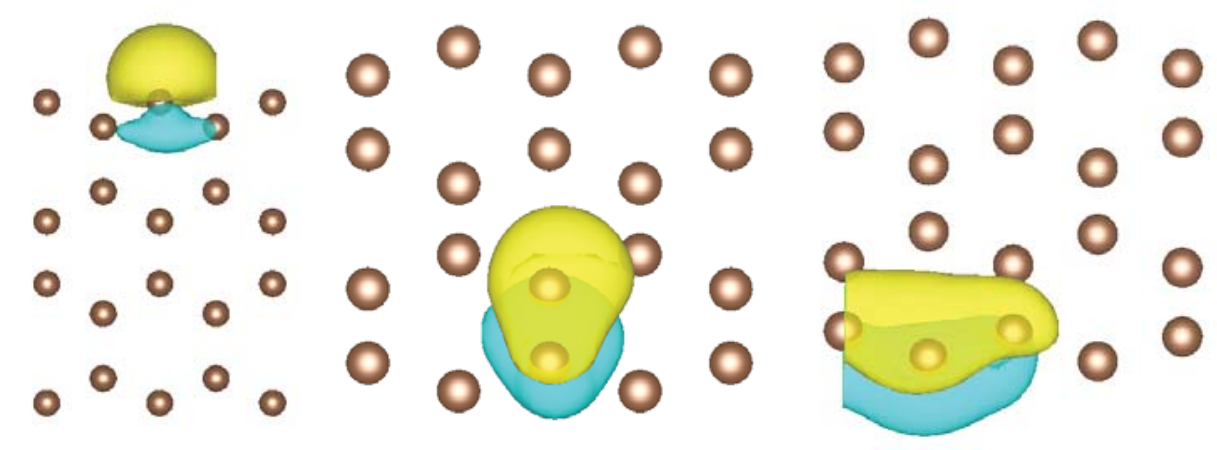

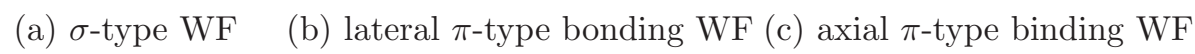

Figure 5.21: Wannier functions in graphene from the edge manifold

\subsubsection{Interpolated band structure and ballistic transport}

In Fig. 5.22(a) the band structure of a $2 \mathrm{zGNR}$ is computed only in the direction of periodicity, since in the other directions it consists of a set of discreet dispersionless levels. According to the Landauer transport theory [55], the conductance ${ }^{6}$ (Fig. 5.22(b)) at any given energy is the sum of quantum conductivities $\left(G_{0}=2 e^{2} / h\right)$ of individual conducting channels, where each conducting channel corresponds to a single energy band, thus ballistic conductance at a given energy is the number of bands intersecting a particular ordinate. Due to the Peierls instability, the band curvature at the edges of BZ is zero, which in turn creates a van Howe singularity at these points. Therefore high spikes on the density of states plot (Fig. 5.22(c)) correspond to the abrupt changes in the ballistic conductance, high density of states causes electron's effective mass to approach infinity, and thus electron mobility and conductance go to zero.

\footnotetext{
${ }^{6}$ The conductance and the transmission function are connected by Eq. (3.16). However, if bias is small or the temperature is $0^{\circ} \mathrm{K}$, the conductance and the transmission function differ only by the factor of $2 e^{2} / h$
} 


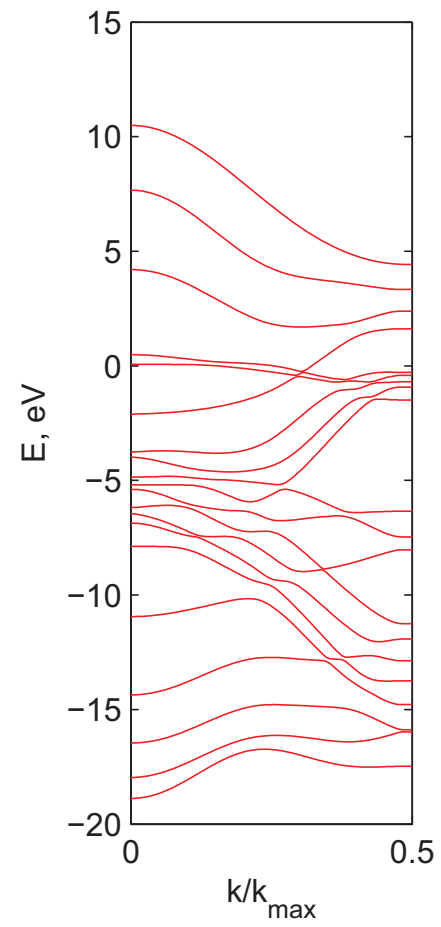

(a)

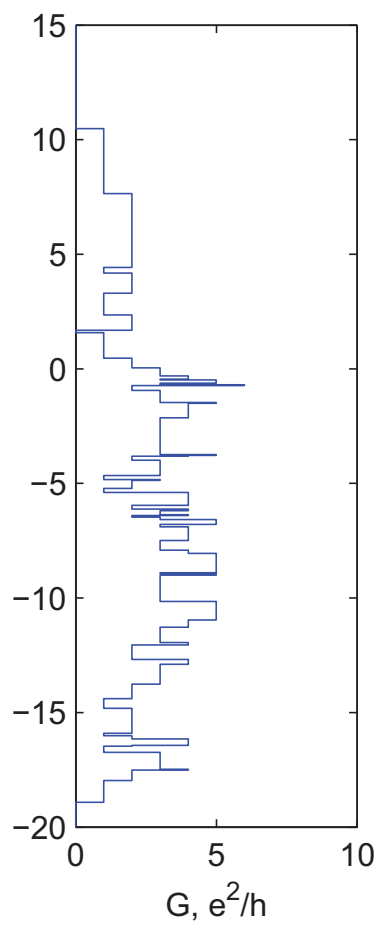

(b)

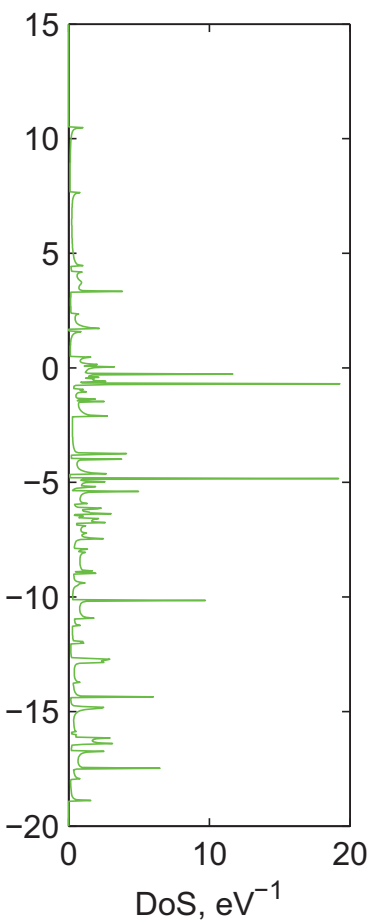

(c)

Figure 5.22: Band structure (a), ballistic transmission function (b), and density of states (c) of a $2 \mathrm{zGNR}$

\subsubsection{Non-equilibrium Green's functions (NEGF) transport}

Pure ballistic regime cannot be observed in the experiment because is assumes infinite periodicity of unit cells and thus does not account presence of scattering centers. Fabrication of GNR devices is usually done by lithographic etching of a graphene sheet [97]. This process leaves large areas of pristine graphene that are used to connect the electrodes. Therefore real transport through a GNR should be considered in a semi-infinite structure, like the one in Fig. 5.23. This structure has open boundaries, meaning that carbon atoms connected with their periodic images in both lateral directions. Then matrix elements of the Hamilton matrices calculated for infinite graphene sheet (in the orthorhombic setting) are matched on the boundaries with the matrix elements of the supercell Hamiltonian $\left(\mathbf{H}_{\mathbf{C}}\right)$, producing Green's func- 


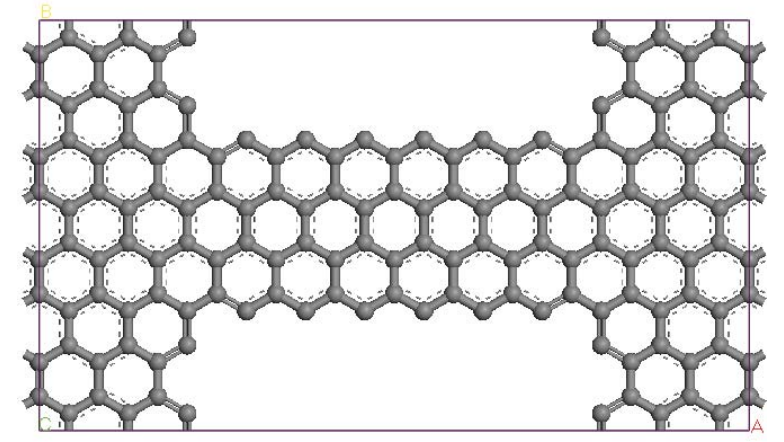

Figure 5.23: Structure of a real GNR device

tions for a narrow GNR strip confined between two "infinite" graphene semiplanes. The same consideration for the choice of the starting WF manifold are made as in case of a one dimensional GNR and infinite graphene sheet. The set of WFs consisted again of binding $\sigma$ and $\pi$ orbitals and antibinding $p_{z}$ and $\pi^{*}$ orbitals. Band structure, conductance, and Density of states (DoS) are shown in Fig. 5.24.
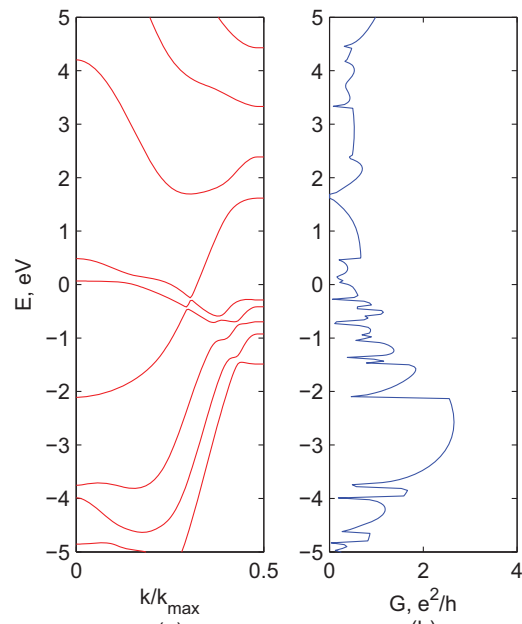

(a)

(b)

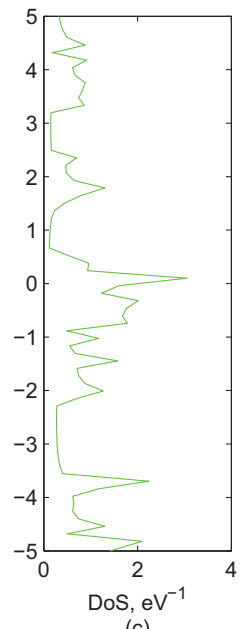

(c)

Figure 5.24: Band structure (a), ballistic transmission function (b), and density of states (c) of a structure in Fig. 5.23

Figure 5.25 shows two overlapping transmission functions (conductance) curves. The solid one corresponds to the ballistic case, and the dashed to the lcr case. In 


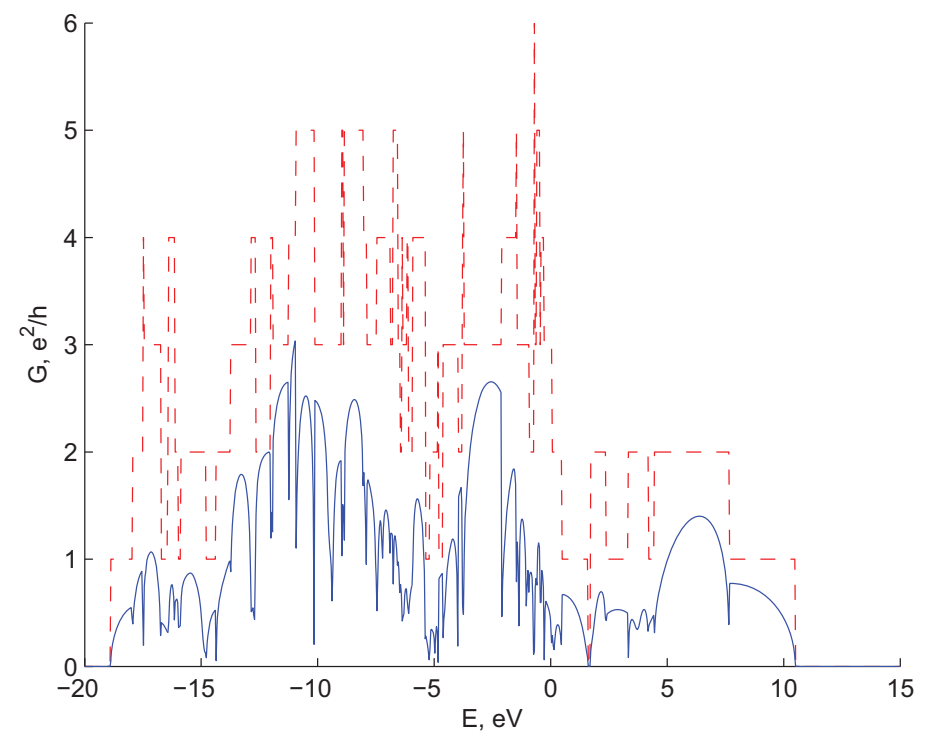

Figure 5.25: Ballistic and coherent conductance for the structure in Fig. 5.23

the ballistic case, again, conductance is simply proportional to the number of bands crossed by energy ordinate, but in the coherent case conductance is calculated from the Eq. (3.16) and Eq. (3.15). However, if a narrow GNR is attached between two semi infinite electrodes, electrons entering the conductor undergo strong backscattering from the edges of the electrodes. Therefore conductance in the coherent regime is always less than in the ballistic, since now electrons in each conduction channel (band) are "penalized" for having momentum close to the BZ edge of the periodic part of the structure.

\subsection{Thermal conductivity of graphene and GNRs}

\subsubsection{Ballistic and semiballistic transport in graphene}

As is has been mentioned in Sec.3.1, electrical and thermal currents depend on the energy spectrum, and the distribution function on the left and right ends of the 
nanodevice, essentially the number of occupied states on both ends:

$$
\begin{aligned}
& I=\frac{1}{h} \int \mathcal{T}(\varepsilon) e\left(f\left(\varepsilon-\mu_{L}\right)-f\left(\varepsilon-\mu_{R}\right)\right) d \varepsilon \\
& Q=\frac{1}{h} \int \Xi(\omega) \hbar \omega\left(\eta\left(T_{\text {hot }}\right)-\eta\left(T_{\text {cold }}\right)\right) d(\hbar \omega)
\end{aligned}
$$

The difference in these two formulas ( $e \leftrightarrow \hbar \omega$ ) comes from the fact that one describes charged particle flux (with charge e), whereas the other one - energy flux (with energy quantum $\hbar \omega)$. Also since electrons are fermions, and phonons are bosons, the occupation number is determined by $\eta(T)$, the Bose-Einstein distribution function.

By analogy with Eq. (3.16) thermal conductance becomes (see e.g. [98]):

$$
\begin{aligned}
& \sigma(T)=\frac{d Q}{d T}=\frac{1}{h} \int \Xi(\omega) \hbar \omega \frac{d \eta}{d T} d(\hbar \omega) \\
& \sigma(T)=\frac{k_{B}^{2} T}{h} \int \frac{x^{2} e^{x} d x}{\left(e^{x}-1\right)^{2}} \Xi\left(x k_{B} T / \hbar\right)
\end{aligned}
$$

In this equation, the total transmission function $\Xi(\omega)=\sum_{m} \Xi_{m}(\omega) \Theta\left(\omega-\omega_{m}^{i}\right) \Theta\left(\omega_{m}^{f}-\right.$ $\omega)$, which according to the Landauer-Büttiker formalism for 1D systems [99], is defined in the purely ballistic regime as the number of phonon modes at any given frequency $\omega . \Xi_{m}(\omega)$ is the mode resolved transmission function, in the ballistic regime each excited mode contributes equally to the thermal conductivity, and thus for purely ballistic transport $\Xi_{m}(\omega)=1$ for all $m$. Analogously to Eq. (3.16), a quantum of thermal conductance can be introduced $[100]^{7}$ :

$$
G_{t h}=\frac{\pi^{2} k_{B}^{2} T}{3 h} .
$$

\footnotetext{
${ }^{7}$ The exact value of the integral was used:

$$
\int_{0}^{\infty} \frac{x^{2} e^{x} d x}{\left(e^{x}-1\right)^{2}}=\frac{\pi^{2}}{3}
$$
}


Within the NEGF formalism, $\Xi(\omega)=\operatorname{Tr}\left[\Gamma^{L} G \Gamma^{R} G^{\dagger}\right]$, where $G$ is now the phonon's Green's function of a nanodevice, $\Gamma$ are the coupling matrices. (see Sec. 3.1) This formalism is applicable to the theory of ballistic transport based on the Landauer formula, where elastic scattering is not addressed.

Conventional approach to include elastic scattering is to consider the Boltzmann transport equation and use various approximations for the collision integral. In the diffusive regime the transmission function can also be described in terms of a characteristic relaxation wavelength, along the axial direction of the GNR:

$$
\Xi_{m}(\omega)=\lambda_{m}(k) / L=v_{m}(k) \tau_{m}(k) / L
$$

Here an implicit assumption is made that mean free path $\lambda$ is less than the length of the conductor $L$. However, if the collision integral in the right hand side of the Boltzmann equation is replaced by any finite lifetime, ballistic transport is entirely excluded from this model, since ballistically transported phonons have infinite lifetime. In the Eq. (5.9), the characteristic lifetime $\tau_{m}(k)$ is better represented as a function of the wave vector, we rewrite Eq. (5.8) as:

$$
\sigma(T)=\sum_{m} \int_{\omega_{m}^{i}}^{\omega_{m}^{f}} \frac{d \omega}{2 \pi} \hbar \omega \Xi_{m}(\omega) \frac{d \eta}{d T}=\frac{1}{2 \pi} \sum_{m} \int_{0}^{a^{*}} \hbar \omega_{m}(k) v_{m}(k) \Xi_{m}(\omega) \frac{d \eta}{d T} d k_{x}
$$

The summation runs over all modes $(m)$, and integration is over one-dimensional Brillouin zone (BZ); $a^{*}$ - distance between $\Gamma$ and BZ boundary in the axial direction (in reciprocal distance units), $\eta$ is the Bose-Einstein distribution function, $v_{m}(k)$ is the phonon group velocity in the axial direction of the ribbon $v_{m}(k)=\frac{d \omega_{m}(k)}{d k}$. The effect of scattering can be included in the transmission function $\Xi_{m}(\omega)$ by introduction of an appropriate Green's function for the system [98, 101]. 
Inserting $\Xi_{m}(k)$ into Eq. (5.8) and using the relation between thermal conductance and thermal conductivity: $\kappa(T)=(L / s) \sigma$, the latter can be expressed as:

$$
\kappa(T)=\frac{L}{s} \frac{\hbar}{2 \pi} \sum_{m} \int_{0}^{a^{*}} \omega_{m}(k) v_{m}(k) \Xi_{m}(k) \frac{d f}{d T} d k_{x},
$$

where $s$ is the thickness of graphene monolayer. This value is typically taken as $3.35 \AA$, the separation between graphene layers in graphite. However, Muñoz et. al. [102] claim that the correct value for this parameter should be $s \approx 0.88 \AA$. This thickness correctly describes the mechanical properties of graphene and is different from the traditionally accepted value $3.35 \AA$ that equals to $c$-spacing in graphite, i.e. a bulk property, controlled by the van der Waals interlayer interaction. This difference, which is referred to as the Yakobson paradox, has been debated in the literature $([102,103,104,105])$. We, however, will use $3.35 \AA$ in order to be consistent with the published literature [102, 106, 107, 108]

The transmission function $\Xi_{m}(\omega)$ cannot be grater than one, which of course, corresponds to pure ballistic transport. This means that the transmission function determined by the Eq. (5.9) should be truncated at unit value. This however, creates an ambiguity, since $\Xi_{m}(\omega)$ exhibits an abrupt break. Therefore, in order to describe "mixed" transport (both diffusive and ballistic transport contributing about the same magnitude to total transport), a quantum tunneling theory should be employed.

Equation (5.10) requires knowledge of full phonon dispersion relation for the GNRs $\omega_{m}(k)$ and the transmission function. Only scattering on rough boundaries is included in the total $\Xi_{m}$ in our consideration. We ignore all other scattering mechanisms, such as isotope scattering, impurity scattering, phonon-electron scattering, and normal and Umklapp phonon-phonon scattering, assuming them to be negligible in comparison to the rough boundary. Further, we discuss the effect of different chiralities on $\Xi_{m}$. 
In the next two sections, we discuss the phonon spectrum in GNRs of different width and chirality and the effect of different chiralities on $\Xi_{m}$.

\subsubsection{Phonon dispersion relations in GNR}

Heat conductance is intimately related to the phonon dispersions through $\omega(k)$ and $v(k)$, hence it is important to understand the phonon spectrum of GNRs. In order to obtain accurate results for the dispersion relations, we employed the full phonon calculation using ab-initio density functional theory (DFT) approach along with a much less computationally intensive force field methods, as often used by other authors (see e.g. [109]). Phonon band structure within the DFT framework was calculated using CASTEP code [110] with the GGA B3LYP [44, 45] parametrization for the exchange-correlation functional. Ab-initio techniques, as implemented (e.g. in CASTEP), allow calculation of phonon dispersion relation without exploiting any empirical parameters. These techniques are much more exact, but usually very costly in terms of computation time. Linear response method was chosen to calculate the dynamical matrix. The main advantage of this scheme is that there is no need to artificially increase the cell size in order to accommodate small values of the q-vectors, as in the frozen phonon method to overcome the long range interaction problem. We have also studied phonon dispersion relations using the force field method as implemented into GULP [111]. Dreiding parameterization of interatomic potential was used for two and three body interaction energy. With this potential, a dynamic matrix of the system was constructed and the dispersion relations for the phonons in graphene were calculated.

The k-path was chosen to be $\Gamma$ - K, for the zigzag terminated ribbons and $\Gamma$ $\mathrm{M}$ for the ribbons with the armchair type of boundary. The agreement between the published experimental results [112], and DFT calculations is very close. CASTEP 


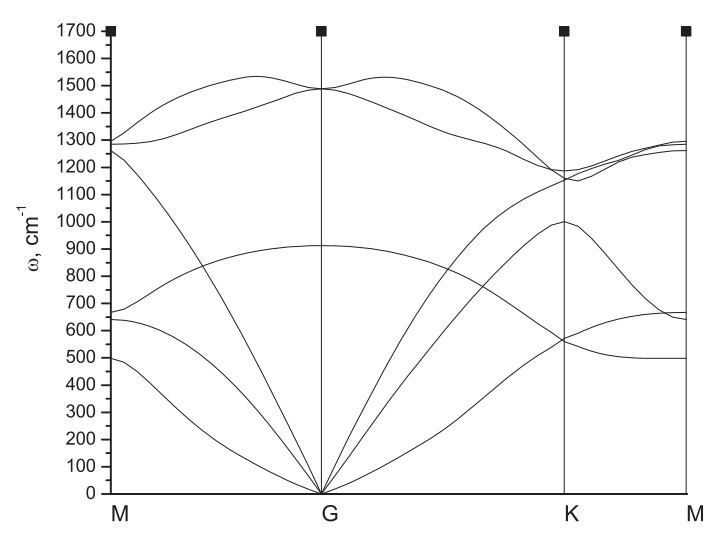

(a)

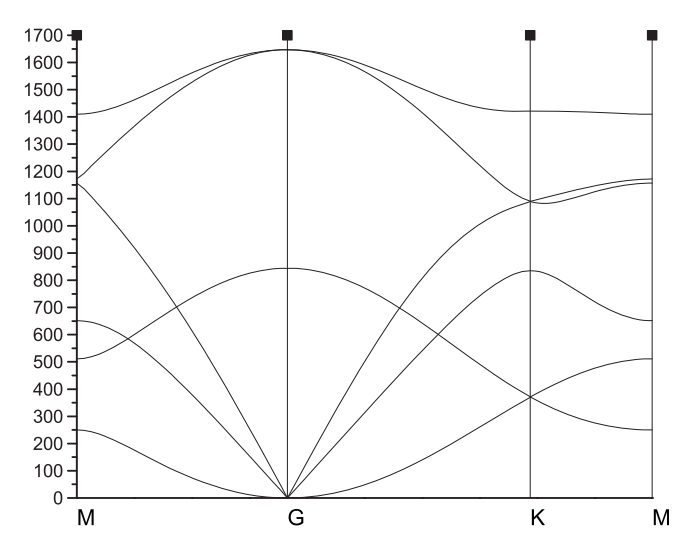

(b)

Figure 5.26: The phonon dispersion branches for a graphene sheet, plotted along high symmetry directions a) calculated using CASTEP b) calculated using GULP

slightly underestimates the phonon energies, especially of the optical modes, by about $4 \mathrm{meV}$ compared to the experimental values. (See Table 5.4) Nevertheless, DFT results still provide a better qualitative description of the phonon spectrum, spectra obtained by means of semi-empirical methods deviate from experimental and ab-inito spectra especially with respect to the LO and TO modes splitting, and in the K-M direction in particular. However, the K-M direction coincides with the honeycomb edge and thus does not correspond to any possible cutting (and thus transport as well) directions, unlike $\Gamma-\mathrm{K}$ and $\Gamma-\mathrm{M}$ directions which correspond to armchair and zigzag chirality respectively. (See Fig. 5.17 and the inset in it)

Table 5.4: Comparison between experimental [112], ab-initio, and semi-empirical phonon spectra

\begin{tabular}{|c|c|c|c|c|c|c|c|c|c|}
\hline & \multicolumn{3}{|c|}{ LO $\left(\mathrm{cm}^{-1}\right)$} & \multicolumn{3}{c|}{ TO $\left(\mathrm{cm}^{-1}\right)$} & \multicolumn{3}{c|}{ LA $\left(\mathrm{cm}^{-1}\right)$} \\
\hline & exp & CASTEP & GULP & exp & CASTEP & GULP & exp & CASTEP & GULP \\
\hline$\Gamma$ & 1583 & 1488 & 1647 & 1565 & 1488 & 1647 & 0 & 0 & 0 \\
$M$ & 1323 & 1275 & 1172 & 1390 & 1285 & 1410 & 1290 & 1262 & 1157 \\
$K$ & 1194 & 1153 & 1050 & 1265 & 1187 & 1419 & 1194 & 1153 & 1090 \\
\hline
\end{tabular}


The phonon dispersion relations obtained were typical for graphene: despite its 2-D nature, graphene has three acoustic phonon modes. The phonon group velocity, calculated as $\partial \omega / \partial k$ at the $\Gamma$ point associated with the LA and TA modes is $1.3 \times 10^{6}$ and $2.2 \times 10^{6} \mathrm{~cm} / \mathrm{s}$, respectively. The out-of-plane mode $(\mathrm{ZA})$ in both directions has a quadratic dispersion relation (and thus has formally zero speed of sound at the $\Gamma$ point). This effect becomes important for thermal conductance at low temperatures. The number of optical modes varies with the size of the supercell. In the case of the infinite graphene sheet, the phonon spectrum in the $\Gamma-\mathrm{M}$ and $\Gamma-\mathrm{K}$ directions, corresponding to the zigzag and armchair chiralities, is presented in Fig. 5.27. It can be seen that the TA mode demonstrates stronger anisotropy than the other acoustic modes.

The number of optical modes varies with the size of the supercell. In the case of the infinite graphene sheet, the phonon spectrum in the $\Gamma-\mathrm{K}$ and $\Gamma-\mathrm{M}$ directions, corresponding to the zigzag and armchair chiralities, is presented in 5.27. It can be seen that the TA mode demonstrates stronger anisotropy than the other acoustic modes.
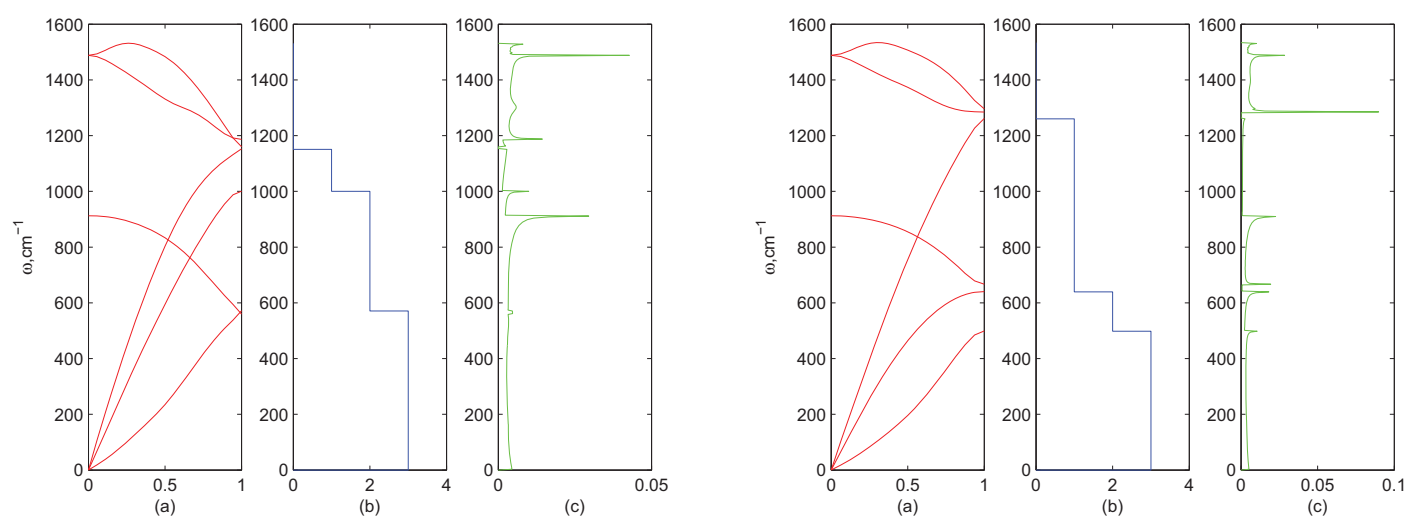

Figure 5.27: (a) Phonon dispersion relations, (b) Ballistic transmission function, and (c) Phonon density of states for the $\Gamma-\mathrm{K}$ (armchair) (left) and $\Gamma-\mathrm{M}$ (zigzag) (right) directions in graphene 
ZA and ZO modes actually possess imaginary frequencies (shown as their absolute value), indicating some instability of the graphene with respect to long wavelength periodic distortion. This phenomenon is widely known as graphene buckling and is inherent in GNRs too [113].

Phonon dispersion relations in GNRs can be obtained from those for infinite 2D graphene in the same way it was done with electronic bands structure (see Eq. (5.7)):

$$
\omega_{\mu}^{G N R}(k)=\omega_{g 2 D}\left(k \frac{\mathbf{K}_{2}}{\left|\mathbf{K}_{2}\right|}+\mu \mathbf{K}_{1}\right),
$$

where $m$ - is a phonon mode, $\mathbf{K}_{1}$ and $\mathbf{K}_{2}, \mathrm{~N}, \mu$ are defined in table 5.3.

Thus, the vibrational frequencies for a ribbon are given by dispersion relations $\omega_{b}=c_{b} \mathbf{q}^{2}, \omega_{L A}=c_{L A} q \omega_{T A}=c_{T A} q \omega_{\tau}=c_{\tau} q_{l}$, for bending (b), longitudinal (LA), transverse (TA) acoustical, and torsion $\tau$ polarizations, $c_{L A}, c_{T A}$, and $c_{\tau}$ represent the usual speeds of sound, with $\mathbf{q}=\left(q_{l}, q_{w}\right)$ and $q=|\mathbf{q}|$, and the quadratic dispersion of the bending mode causes its speed of sound to depend explicitly on the wavelength as $\sim c_{b}|\mathbf{q}|$ [102]. Equation (5.11), by derivation, does not incorporate the torsion edge mode of GNR. However, $c_{\tau}$ for the torsion mode vanishes as $\sim 1 / w$ in the limit of a wide ribbon and therefore can be ignored for the simplicity of the calculation.

Therefore within this approximation all dispersion laws and transport properties are already incorporated into the phonon spectrum of infinite graphene sheet. There are, however, some exceptions from that simple rule [114]. Therefore we have also calculated dispersion relations for GNRs explicitly using GULP in order to estimate the degree of deviation of phonon spectra of GNRs relative to infinite graphene sheet.

The atomic structures consisted of 1D periodic ribbons with unit cell oriented in the direction of repetition of $2.572 \AA$. The width of the ribbons of each chirality has been varied from two cells, (a,z)GNR-2 to six cells, (a,z)GNR-6. Vacuum layers 
separating neighboring ribbons were chosen to be $71.5 \AA$ in the lateral direction and $30.6 \AA(10 c)$ in the axial direction.

The BZ path for phonon dispersion calculation has been chosen to be from $\Gamma-\Sigma$ - X (or $\Gamma-\Delta-\mathrm{Y}$ ) with $29 \mathrm{k}$-points on the sampling interval.

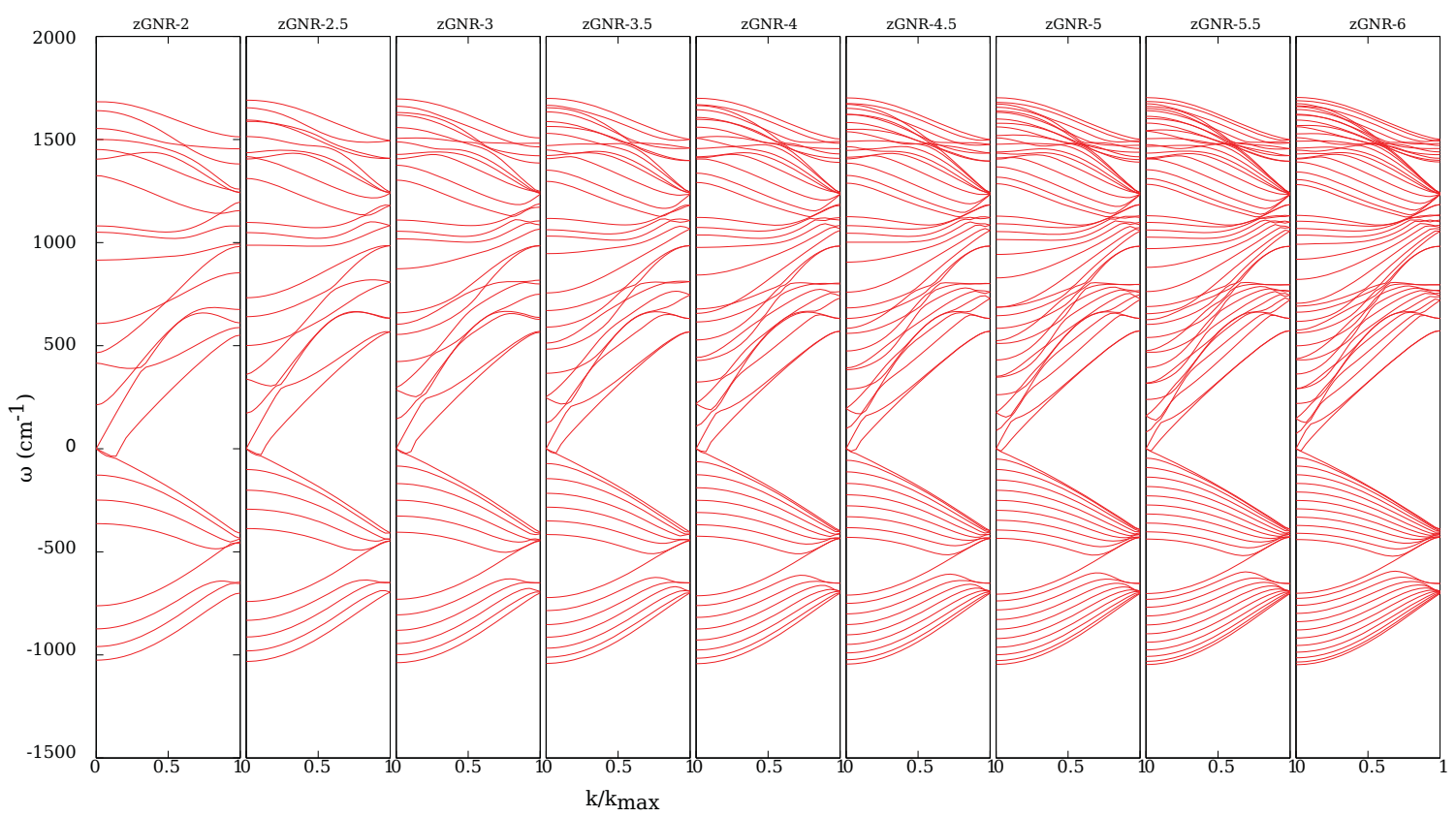

Figure 5.28: Dispersion relations for zigzag GNRs

Dispersion laws in the GNR demonstrate same patterns as those for infinite graphene with some peculiarities. The acoustical branches of the GNRs (LA, TA, ZA) are described satisfactorily in the infinite sheet model. The phonon group velocities associated with the LA and TA modes do not undergo a major change, whereas ZA starts growing dramatically after $\mathrm{N}=4$. This effect will be addressed separately in a future publication. Therefore all further calculations are performed using the infinite graphene sheet dispersion spectrum. 


\subsubsection{Scattering mechanisms in GNRs and the effect of chirality on ther- mal conductivity of GNRs}

From Eq. (5.10) one can see that the only difference in thermal in incorporated in the phonon dispersion law $\omega(k)$ (speed of sound $v(k)$ follows from it as well) and the transmission function. At low temperatures, only acoustic modes with low cut-off frequencies are excited. However, the speed of sound (and the dispersion relations) in the vicinity of the $\Gamma$ point are the same in all lateral directions. The values calculated from spectra represented in Fig. 5.27 are summarized in Table 5.5.

Table 5.5: Speed of sound calculated using ab-initio, and semi-empirical phonon spectra

\begin{tabular}{ccccccc}
\hline & \multicolumn{2}{c}{ ZA $\left(\mathrm{cm}^{-1}\right)$} & \multicolumn{2}{c}{$\mathrm{LA}\left(\mathrm{cm}^{-1}\right)$} & \multicolumn{2}{c}{ TA $\left(\mathrm{cm}^{-1}\right)$} \\
\hline & $\Gamma-\mathrm{K}$ & $\Gamma-\mathrm{M}$ & $\Gamma-\mathrm{K}$ & $\Gamma-\mathrm{M}$ & $\Gamma-\mathrm{K}$ & $\Gamma-\mathrm{M}$ \\
\hline CASTEP & 976.49 & 964.59 & 3698.17 & 3706.24 & 5923.37 & 5926.10 \\
GULP & 4.53 & 4.94 & 3019.57 & 3022.24 & 5007.04 & 5011.32 \\
\hline
\end{tabular}

By inspection of the first column we can see a clear discrepancy in the ZA mode as calculated by CASTEP and GULP. The reason for that is that the ZA mode corresponds to the low graphene buckling, which can be properly addressed in large supercells only, however, because of high computation cost of CASTEP calculations, the structure for ab-initio examination was limited by only one unit cell; GULP in turn is free from that constrain and that is why GULP calculations reproduce ZA mode more appropriately.

The quadratic dispersion law for the ZA branch is a characteristic feature of layered crystals [115] and can be explained as a consequence of hexagonal graphene symmetry [114], compared to the presence of the Dirac point that follows from the same symmetrical consideration as well.

If the dispersion spectrum at low energies near the $\Gamma$ point is the same for both 
cut directions, the difference in their thermal conductivity is entirely governed by the difference in their transmission functions. As it follows from Eq. (5.9), the transmission function can be expressed in terms of relaxation times for different processes. The total (branch specific (j), momentum dependent $(\mathbf{q})$ ) relaxation time is obtained by the Mathiessen rule:

$$
\frac{1}{\tau_{j}(\mathbf{q})}=\frac{1}{\tau_{j, N}(\mathbf{q})}+\frac{1}{\tau_{j, U}(\mathbf{q})}+\frac{1}{\tau_{j, I}(\mathbf{q})}+\frac{1}{\tau_{j, B}(\mathbf{q})}
$$

where we include into consideration normal (N) and umklapp (U) three-phonon scattering, isotope scattering (I), and boundary surface-roughness scattering (B). The dependence of $\mathrm{N}$ and $\mathrm{U}$ relaxation times on the phonon energy and lattice temperature is given by (see e.g. [109]):

$$
\frac{1}{\tau_{j, N / U}^{L A / T A}(\omega)}=\frac{\hbar \gamma_{j}^{2}}{\bar{M} \Theta_{j} \bar{v}_{j}^{2}} \omega_{j}^{n} T e^{-3 T / \Theta_{j}}
$$

where $\mathrm{n}=2$ for normal scattering and $\mathrm{n}=4$ for umklapp scattering, $\Theta_{j}$ is branch-specific Debye temperature $^{8}, \bar{M}$ is the average atomic mass, $\bar{v}_{j}$ is the speed of sound. The strength of the phonon-phonon scattering process for each branch is controlled by the Grüneisen constant which is deduced from the logarithmic derivative of dispersion with respect to volume $\gamma_{j}=\left(-a / 2 \omega_{j}(q)\left(d \omega_{j}(q) / d a\right)\right)$. Scattering from the mass differences due to the presence of naturally occurring isotopes can be represented by

\footnotetext{
8$$
\Theta_{j}^{2}=\frac{5 \hbar^{2}}{3 k_{B}^{2}} \frac{\int \omega^{2} g_{j}(\omega) d \omega}{\int g_{j}(\omega) d \omega}
$$

where the vibrational density of states function (vDOS) $g_{j}(\omega)=\sum_{q} \delta\left(\omega-\omega_{j}(q)\right)$. This way, the temperature dependence of the contribution of each phonon branch to the total thermal conductivity is correctly represented.
} 
an energy- dependent rate

$$
\frac{1}{\tau_{I}(\omega)}=\Gamma \Omega_{0} / 12 \omega^{2} g(\omega)
$$

with the total vDOS $g(\omega)=\sum_{j} g_{j}(\omega)$. The mass difference constant that governs the relative strength of this scattering mechanism is given by the sum over all naturally occurring isotopes weighted by their mass $M_{i}$ relative to the average mass: $\Gamma=$ $\sum_{i} f_{i}\left(1-M_{i} / \bar{M}\right)^{2}=c(1-c) /(12-c)^{2}$. Using natural abundances of ${ }^{12} \mathrm{C}$ and ${ }^{13} \mathrm{C}$ of $98.9 \%$ and $1.1 \%$, respectively, we obtain $\mathrm{c}=0.011$, i.e. $\Gamma=0.000076$.

The isotope scattering process can be neglected in our consideration, and the normal and umklapp scattering mechanisms are insignificant at low temperatures, thus all anisotropy in thermal conductance of GNRs at low temperature is due to the boundary scattering.

There exist two approaches for calculating scattering due to roughness of the surface. Both employ a specularity parameter $p(k)$, such that $0<p(k)<1$, which represents the probability that a phonon mode $\mathbf{k}$ will be scattered from the rough edge and is proportional to the Fourier transform of the surface roughness correlation function [101]. The conventional approach relates surface roughness with phonon lifetime $[116]$

$$
\tau_{m}(k)=\left(\frac{1+p(k)}{1-p(k)}\right) \frac{W}{v_{m, \perp}(k)}
$$

where $W$ is the width of the ribbon and $v_{m, \perp}(k)$ is the component of the phonon velocity of mode $k$ in branch $m$ perpendicular to the idealized smooth edge of the nanoribbon. This approximation is valid for pure diffusive transport only. Application of this approach for very short GNRs with "mixed" transport leads to truncation of the transmission function $\Xi_{m}(\omega)$ and may cause abrupt hopping between different 
transport regimes [117]. In contrast to that, the transmission function approach, which comes from the Green's function method (see below) has unity as a limiting case for very small scattering rates.

\subsection{Edge roughness function}

Since scattering on the boundaries is an elastic process, it should be addressed by means of the Green's function formalism. The effect of scattering can be included in the mode transmission function $\Xi_{m}(\omega)$ by introduction of an appropriate Green's function for the system. The theory of Green's function in application to boundary scattering was developed by Santamore et.al. in [101]. However, they had limited their consideration by uncorrelated roughness, whereas in GNRs the shape of the boundary is regular and thus we should develop an approach to account for correlated roughness. In this study we will explicitly account for different chiralities and modify the surface roughness functions accordingly.

Phonon scattering at the boundaries is a dominant mechanism limiting the mean free path in narrow GNRs. We will consider a GNR as a 2D waveguide-like-structure extended in the axial $(x)$ direction and bounded at $y= \pm W / 2$, where $\mathrm{W}$ is the width of a GNR. This condition describes a macroscopically smooth boundary. We account for effects of atomistically terminated boundaries by introducing roughness functions $f_{ \pm}(x)$, so that rough boundaries are described by $y= \pm W / 2+f_{ \pm}(x)$. Moreover, top and bottom functions are correlated and describe same type of termination: $f_{-}(x)=$ $f_{+}(x)$. Offset of one boundary relative to the other will manifest itself in the Fourier transform (see below) as a phase factor, which becomes unity in the power spectrum, and thus can be considered together with non-offset case.

The roughness functions are periodic with the lattice period of the nanoribbon: 
$\sqrt{3} a_{0}$ for the zigzag GNR and $3 a_{0}$ for armchair, $a_{0}$ is the C-C distance. Thus $f_{ \pm}(x)$ can be expressed in terms of a single cell:

$$
f_{ \pm}(x)=\sum_{n=-\frac{N-1}{2}}^{\frac{N-1}{2}} K\left(x+a_{0} m n\right)
$$

where $K$ is the shape function of either a zigzag or armchair boundary (Fig. 5.29) and $m$ is the factor that determines the period, i.e., $\sqrt{3}$ for the zigzag GNR and 3 for armchair, $N$ - number of unit cells along the direction of heat propagation $(x)$. The Fourier transform of the roughness function yields ${ }^{9}$ :

$$
\mathcal{F}\left[f_{ \pm}(x)\right](k)=\mathcal{F}[K(x)] \frac{\sin \left(N \pi m a_{0} k\right)}{\sin \left(\pi m a_{0} k\right)},
$$

where $\mathcal{F}[K(x)]$ is the Fourier transform of a single structural feature's shape function. The functions $K$ are defined as:

$$
\begin{gathered}
K_{a}(x)=\left\{\begin{array}{cl}
0 & \text { if } a_{0} \leq x \leq-a_{0} ; \\
\sqrt{3}\left(x+a_{0}\right) & \text { if }-a_{0} \leq x \leq-\frac{a_{0}}{2} ; \\
\sqrt{3} a_{0} / 2 & \text { if }-\frac{a_{0}}{2} \leq x \leq \frac{a_{0}}{2} ; \\
\sqrt{3}\left(a_{0}-x\right) & \text { if } \frac{a_{0}}{2} \leq x \leq a_{0} .
\end{array}\right. \\
K_{z}(x)=\left\{\begin{array}{cl}
0 & \text { if } \frac{\sqrt{3}}{2} a_{0} \leq x \leq-\frac{\sqrt{3}}{2} a_{0} ; \\
a_{0} / 2+x / \sqrt{3} & \text { if }-\frac{\sqrt{3}}{2} a_{0} \leq x \leq 0 ; \\
a_{0} / 2-x / \sqrt{3} & \text { if } 0 \leq x \leq \frac{\sqrt{3}}{2} a_{0} .
\end{array}\right.
\end{gathered}
$$

\footnotetext{
${ }^{9}$ We use the "crystallographic" definition of the Fourier transform, i.e. $\mathcal{F}[f(x)]=$ $\int_{-\infty}^{+\infty} f(x) \exp (-2 \pi i k x) d x$
} 
Thus the Fourier transforms of $K_{a}$ and $K_{z}$ are:

$$
\begin{aligned}
& \mathcal{F}\left[K_{a}\right]=\frac{\sqrt{3} a_{0}^{2}}{4} \operatorname{sinc}\left(3 / 2 a_{0} k\right) \operatorname{sinc}\left(1 / 2 a_{0} k\right) \\
& \mathcal{F}\left[K_{z}\right]=\frac{a_{0}^{2}}{4} \operatorname{sinc}\left(\sqrt{3} a_{0} k\right)^{2}
\end{aligned}
$$

The Fourier transform of the autocorrelation function is:

$$
S(k)=\left|\mathcal{F}\left[f_{ \pm}(x)\right]\right|^{2}=|\mathcal{F}[K]|^{2} \frac{\sin ^{2}\left(N \pi m a_{0} k\right)}{\sin ^{2}\left(\pi m a_{0} k\right)}
$$

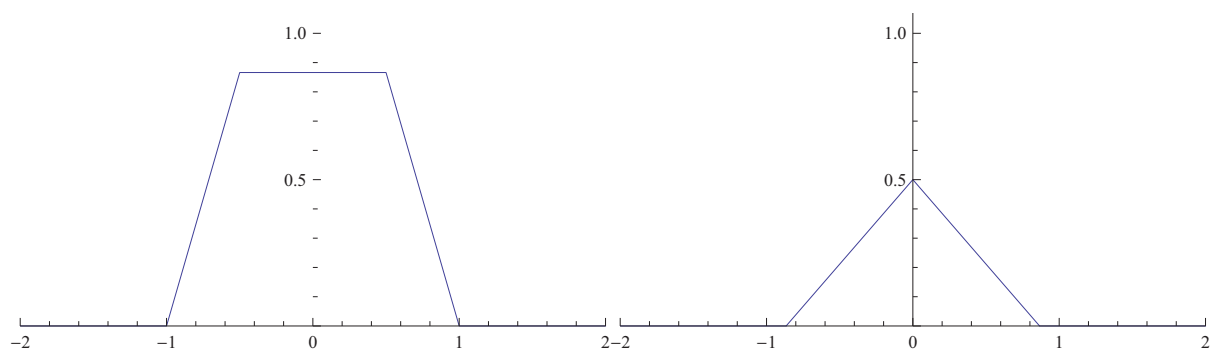

(a)

(b)

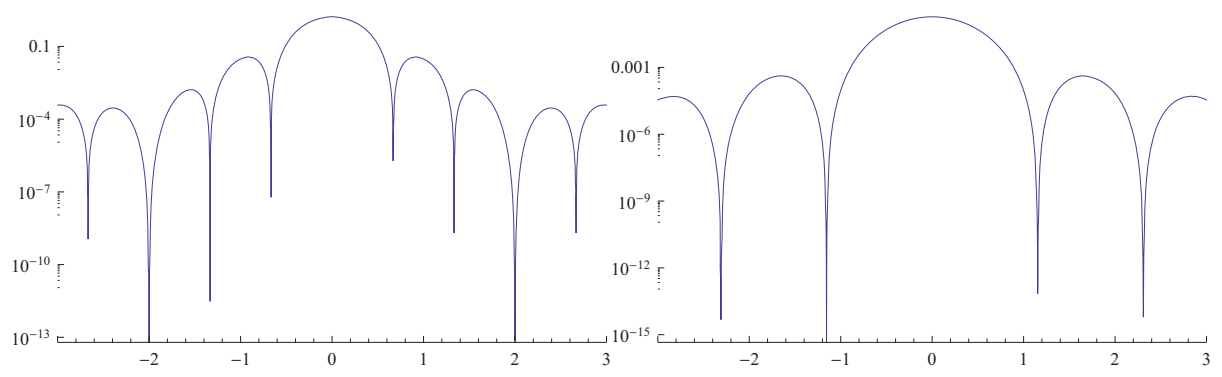

(c)

(d)

Figure 5.29: Shape functions and their Fourier transforms. a) Shape function for the armchair boundary, b) Shape function for the zigzag boundary c) Fourier transform of the shape function for the armchair boundary, d) Fourier transform of the shape function for the armchair boundary

These roughness functions $S(k)$ deviate from the diffuse scattering limit due to high symmetry and regularity (i.e. periodicity) of the boundaries. As $N \rightarrow \infty$, which corresponds to a very long ribbon, $S(k)$ turns into an impulse train and thus 
cannot be approximated by any continuous function, but averaging over all unit cells eliminates this discrepancy. In Eq. (5.14) the Fejér kernel term ${ }^{10}$, after averaging over one period, produces the factor $N$, equal to the number of unit cells. Since $N=L /\left(m \cdot a_{0}\right)$, the edge roughness correlation function can be expressed as:

$$
\begin{aligned}
& \left\langle S_{a}(k)\right\rangle=\frac{a_{0}^{3} L}{16} \operatorname{sinc}^{2}\left(\frac{3}{2} a_{0} k\right) \operatorname{sinc}^{2}\left(\frac{1}{2} a_{0} k\right) \\
& \left\langle S_{z}(k)\right\rangle=\frac{a_{0}^{3} L}{16 \sqrt{3}} \operatorname{sinc}^{4}\left(\sqrt{3} a_{0} k\right)
\end{aligned}
$$

\subsubsection{Scattering on rough boundaries}

The Green's function method based on the quantum mechanical approach involves computation of the scattering amplitude between different modes [101]. The equation for the energy flux scattering probability in this model is:

$$
t_{ \pm n m}(k)=\frac{N_{n}^{2} N_{m}^{2}}{4 K_{n} K_{m}}\left(k^{2} \mp K_{n} K_{m}\right)^{2}\left\langle S\left(K_{n} \mp K_{m}\right)\right\rangle,
$$

where $N_{p}=\frac{1}{\sqrt{W}}$ if $p=0$ and $\frac{2}{\sqrt{W}}$ if $p \neq 0, K_{p}=\sqrt{k^{2}-\frac{p^{2} \pi^{2}}{W^{2}}}$, and $n$ and $m$ are different phonon modes. Because of their small group velocity, optical phonons are often excluded from the computation of thermal transport [118]. Indeed, they carry a negligible amount of heat, however they scatter acoustic phonons, which largely contribute to heat transport. Thus phonons from the three acoustical modes: LA, TA, and ZA can scatter off each other and optical phonons. The number of optical phonons depends on the size of the supercell, $3 N-3$, where $N$ is the number of atoms, but the number of acoustical branches always stays the same. Thus the whole effect of ribbon width is due to scattering of acoustical phonons off of optical ones.

\footnotetext{
${ }^{10}$ Original definition of the Fejér kernel $F_{n-1}(x)=\frac{\sin ^{2}(n x / 2)}{\sin ^{2}(x / 2)}$, the Fejér kernel has a property: $\frac{1}{2 \pi} \int_{-\pi}^{\pi} F_{n}(x) d x=1$
} 
As it has been shown [101] a wave entering mode $m$ has four possible outcomes: (a) after a scattering event it may stay in mode $m$ propagating forward, (b) it may be converted into mode $n$ also propagating forward, (c) it may stay in $m$ mode propagating backwards and, (d) it may be finally converted into mode $n$ propagation backwards. The former two cases do not change heat transport since each mode at frequency $\omega$ contributes the same amount to conductance (see Eq. (5.8)). Thus only backscattering contributes to deviation of the transport coefficient from the ballistic case. This deviation can be characterized by an attenuation coefficient per length of the scattering waveguide (GNR in our case), which can be expanded as:

$$
\begin{aligned}
\gamma_{m}(k) & =\frac{2}{L} \sum_{n} t_{-n m}(k)=\frac{2}{L}\left(\sum_{n \in a c c} t_{-n m}(k)+\sum_{n \in o p t} t_{-n m}(k)\right) \\
& \approx \frac{2}{L} \sum_{n=L A, T A, Z A} t_{-n m}(k),
\end{aligned}
$$

where the first term is responsible for scattering of acoustic phonons from the boundaries into other acoustic modes, whereas the second term is responsible for the scattering into optical modes. The contribution from optical phonons to total thermal conductivity is considered to be negligible (see e.g. $[118,116])$ and therefore optical modes are excluded from Eq. (5.17). The factor of 2 accounts for scattering from both top and bottom surfaces. The attenuation coefficient gives an exponential decay rate of the wave in mode $m$, so that the transmission function of a GNR of length $L$ is:

$$
\Xi_{m}(\omega)=e^{-\gamma_{m} L}
$$

which should be inserted into the Eq. (5.10)

It is worthwhile to point out that equations (5.10) and (5.16) do not contain any material specific information, i.e. they do not depend on the exact dispersion law. 
The total heat conductance at a given temperature is now given by:

$$
\kappa(T)=\frac{L}{s} \frac{\hbar^{2}}{k T^{2}} \sum_{m=a c c} \frac{1}{2 \pi} \int_{0}^{a^{*}} e^{-\gamma_{m} L} v_{m}(k) \frac{\omega_{m}^{2}(k) e^{\frac{\hbar \omega_{m}}{k T}}}{\left(e^{\frac{\hbar \omega_{m}}{k T}}-1\right)^{2}} d k_{x}
$$

\subsubsection{Thermal conductance of GNRs}

In the traditional approach of modeling surface roughness and GNR edges, the roughness correlation function in momentum space is approximated by a Gaussian $[116,101],\left\langle S_{g}(k)\right\rangle=\delta^{2} \sqrt{\pi} b \exp \left(-b^{2} k^{2} / 4\right) L$ where $\delta$ is the roughness amplitude, $a$ is

the correlation length, $\mathrm{L}$ is the length of the sample. In our case, $\delta$ corresponds to the height of the armchair or zigzag boundary terminations, which is proportional to $a_{0}$, and $b$ - to the width of those features, which in turn are proportional to the unit cell length. Thus the correlation function can be expressed in terms of the lattice parameter only. Now by inserting equations (5.15b) into equations (5.16) and (5.17) we can calculate the energy flux scattering probabilities $t_{-n, m}$ and the conductance attenuation coefficients $\gamma_{m}$. Those coefficients for the first three acoustic modes $(m=0$ (LA), 1(TA), 2(ZA)) are shown in Fig. 5.30 as a function of the wave vector.

The obvious difference is embodied in periodicity of the roughness function. Thus the transmission coefficient also appears to be periodic. Indeed, this confirms the intuitive idea that phonons having the same or multiple momentum as the spatial frequency of the scattering boundary will be scattered more efficiently, than those with momentum that does not match.

Also transport clearly becomes ballistic for very short wavelength phonons (high k), which has been discussed by Ziman [116]. In the case of Gaussian roughness, this limit occurs at much shorter wavelengths due to the implicit assumption of complete randomness of the boundary, whereas on the microscale, boundaries in graphene are 


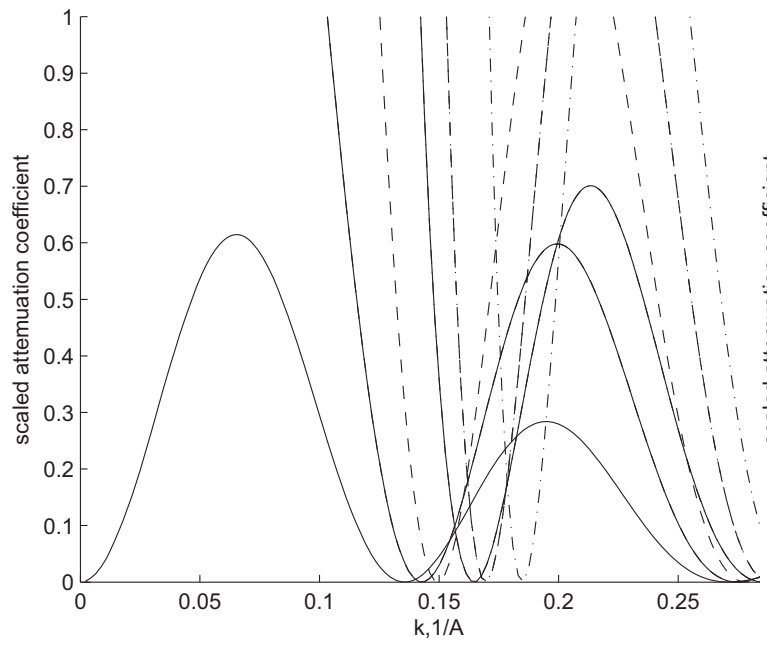

(a) armchair

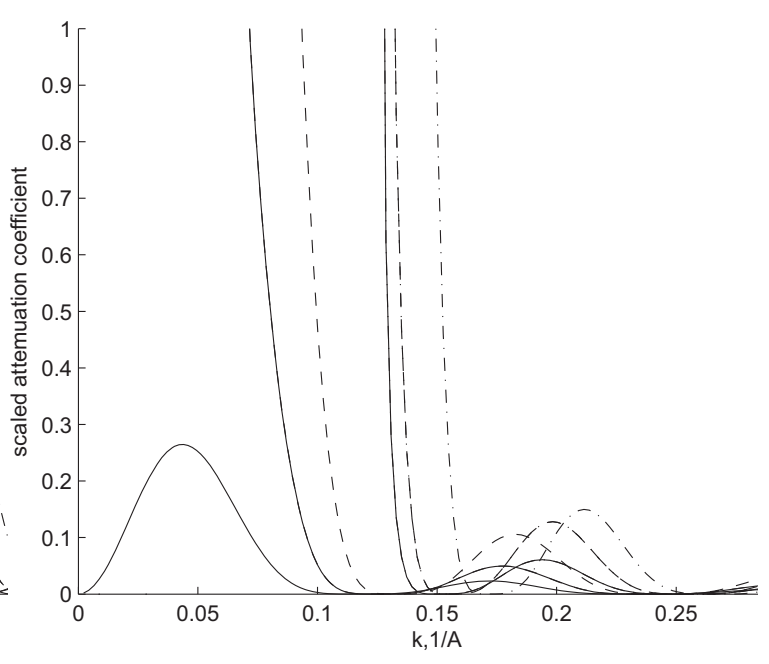

(b) zigzag

Figure 5.30: Scaled attenuation coefficient $\frac{m W^{4}}{a^{3} L} t_{-n, m}$ as a function of k-vector: solid - from mode $\mathrm{m}=0$ to mode $-\mathrm{n}, \mathrm{n}=0 \ldots 2$, dashed - mode $\mathrm{m}=1$ to mode $-\mathrm{n}, \mathrm{n}=0 \ldots 2$, dashed-dotted $-\mathrm{m}=2$ to mode $-\mathrm{n}, \mathrm{n}=0 \ldots 2$.

in fact regular. Thus, phonons with wavelengths much shorter than edge structural features will not scatter off them.

The width dependence is explicitly included into Eq. (5.16), and also implicitly into the dispersion relations, but in this consideration the latter effect is ignored. As the width of GNR grows, the transport becomes more ballistic, and thermal conductance is approaching its limit determined by Eq. (5.8). In fact, comparing the $\sigma-T$ curves for GNRs of width more than $500 \AA$ and with $\Xi(\omega)=1$, one can see that scattering on the boundary is almost negligible, i.e. phonons are traveling essentially ballistically. Similar results were obtained by Evans et.al. [108] by means of the Green-Kubo formula. Their results slightly differ from those presented here. We believe this is due to the fact that we ignored the normal and Umklapp scattering mechanisms (which depend on the GNR size through the Grüneisen parameter), focusing on boundary scattering. 


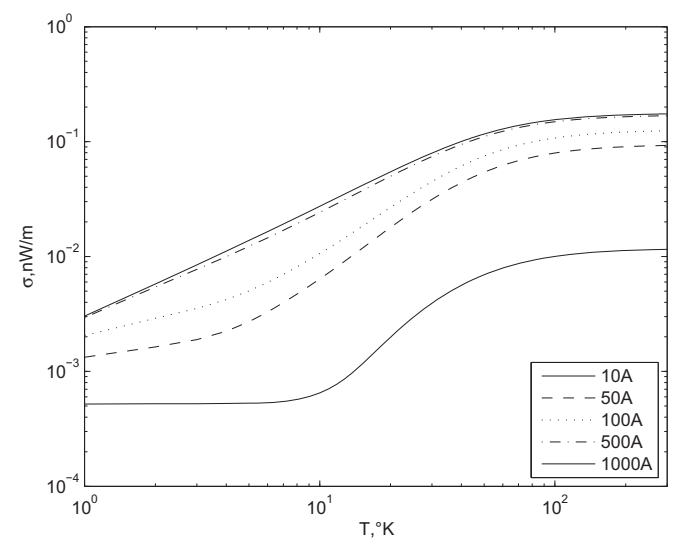

(a) armchair

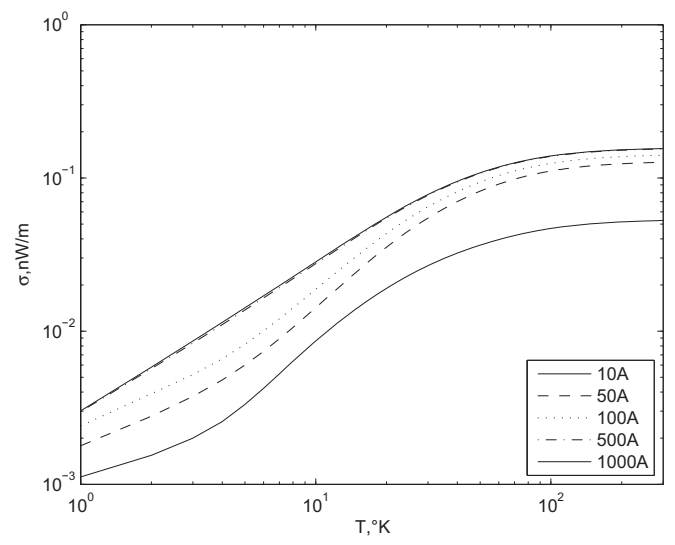

(b) zigzag

Figure 5.31: Thermal conductivity as function of temperature

As can be seen from the plots of thermal conductance versus temperature, for very broad ribbons, edge scattering is negligible, since the majority of phonons are traveling through the central region of GNRs. In this limit the difference between chiralities of GNRs is manifested only in the dispersion curves: high temperature thermal conductance limit of the zGNR is $0.15 n W / K$ and $0.18 n W / K$ for the aGNR, or, for thermal conductivity, 4641 and $5266 \mathrm{~W} /(\mathrm{mK})$, respectively for $10 \mu \mathrm{m}$ long GNRs, which are in good agreement with experimental data [119]. However, when boundary scattering is taken into account, thermal conductance of both types of GNRs degrades, dropping faster for the zGNRs. The reason for this is that $\left\langle S_{z}(k)\right\rangle$ has higher period than $\left\langle S_{a}(k)\right\rangle$ and thus $t_{a}=0$ for more k-values than $t_{z}$, making phonon transport in the aGNRs on average more ballistic.

Limiting cases of very wide and very narrow nanoribbons. In this paragraph two limiting cases of heat transport in GNRs are considered: very wide and very narrow GNRs. The definition of a "very wide nanoribbon" follows from the definition of $K_{p}$ in Eq. (5.16). Neglecting the second term under the square root sign with 
respect of the first one (if $k \gg 1 / W$ ) one obtains $K_{p}=k^{11}$. In this case Eq. (5.16) for the forward scattered modes simplifies as:

$$
t_{+n m}(k) \approx \frac{1}{W^{2}}\left(k^{2}-k^{2}\right)^{2}\langle S(k-k)\rangle \approx 0
$$

and for the backscattered modes:

$$
t_{-n m}(k) \approx \frac{\frac{1}{W^{2}}}{4 k^{2}}\left(k^{2}+k^{2}\right)^{2}\langle S(k+k)\rangle \approx \frac{1}{4} \frac{k^{2}}{W^{2}} a_{0}^{3} L \underbrace{\operatorname{sinc}\left(a_{0} k\right)}_{\approx 0} \approx 0 .
$$

Therefore, as expected, edge scattering is negligible in very wide GNRs, and other "bulk" scattering mechanisms dominate over it.

A "very narrow nanoribbon" is defined as $k \ll 1 / W$, then $K_{p}=-\frac{p \pi}{W}$, and Eq. (5.16) simplifies as:

$$
t_{ \pm n m}(k) \approx \frac{\frac{1}{W^{2}}}{\frac{n m \pi^{2}}{W^{2}}}\left(\mp \frac{n m \pi^{2}}{W^{2}}\right)^{2}\left\langle S\left(\frac{a_{0} \pi}{W}(-n \mp m)\right)\right\rangle \approx \frac{n m \pi^{2}}{W^{4}} a_{0}^{3} L\left(\frac{a_{0} \pi}{W}\right)^{4}
$$

The transition width of a GNR which corresponds to the crossover between two aforementioned regimes can be obtained by considering of the condition for vanishing scattering $t_{ \pm n m}(k) \rightarrow 0$, which follows from Eq. (5.21):

$$
t_{ \pm n m}(k) \approx \frac{\pi^{2}}{W^{4}} a_{0}^{3} L\left(\frac{a_{0} \pi}{W}\right)^{4} \rightarrow 0 \Leftrightarrow W^{8} \gg \pi^{6} a_{0}^{7} L
$$

Taking $\pi^{6} \approx 10^{3}, a_{0}^{7} \approx 10 \AA^{7}, L \approx 10^{5} \AA$, the critical width becomes: $W \gg 10-100 \AA$. That means that for GNRs of the width $W \approx 100-1000 \AA=10-100 \mathrm{~nm}$ the edge scattering becomes negligible comparing to the other "bulk" mechanisms. This result is in very good correspondence with our calculations presented in Fig. 5.31 and is

\footnotetext{
${ }^{11}$ In any case $W \gtrsim a_{0}$
} 
reasonably close to the values obtained by Evans et.al. [108]. 


\section{CHAPTER VI}

Covalent functionalization of graphene 
Chemical modification of graphene by covalent functionalization of its surface potentially allows a wider flexibility in engineering of the electronic structure, in particular, the local density of states of the carbon atoms bound to the modifier, that can result in opening of a band gap. Such binding can involve covalent hydrogenation of graphene to modify hybridization of carbon atoms from $s p^{2}$ to $s p^{3}$ geometry [120, 121, 81]. Methods have also been developed to functionalize graphene covalently with molecular species [28, 29, 122, 123, 124]. Among these, perfluorophenylazide (PFPA) functionalization of graphene is well-developed using a nitrene intermediate. Films of this molecule to act as adhesion layers to produce long ribbons of exfoliated graphene [123, 124, 125].

\subsection{Covalent functionalization of graphene.}

A great deal of interest in covalent functionalization of graphene was generated by the idea that functionalized graphene can act as a sensor by changing its electronic properties (conductivity at first), depending on the number and the type of adsorbed molecules. Some initial attempts have already been made to induce charge carriers into graphene by means of the adsorption of various gas molecules including $\mathrm{NH}_{3}$, $\mathrm{H}_{2} \mathrm{O}$, and $\mathrm{NO}_{2}$ and to study how it affects the DoS $[126,127,81]$, which is related to conductivity, EELS and XPS spectra. Primary interest in any functionalization depends on whether a functionalizing molecule acts like an electron donor, or like an electron acceptor, i.e., if the charge of an atom bound to a graphene sheet is increased or decreased compared to the functionalizing molecule in the free state.

Despite a broad analysis of the DoS and band structure of graphene sheets functionalized with "small molecules" (like $\mathrm{NH}_{3}, \mathrm{H}_{2} \mathrm{O}, \mathrm{NO}_{2}, \mathrm{NO}, \mathrm{CO}[126,127,128]$, transition metals [129]), and "large molecules" (like porphyrines and buckyballs [28], 
azido-trimethyl-silane [29], azomethine [122]), very little theoretical [130, 65, 131] and experimental $[16,132]$ attempts have been made to study electrical conductance of those structures.

The choice of heteroatoms (labeled as $\mathrm{X}$ in tables and formulas) for covalent functionalization of graphene is limited by their electronic structure: hydrogen and halogen atoms have only one vacancy in their electron shell and thus can bind to $p_{z}$ orbitals, the only binding spot for halogens can be on the edge of a GNR.

Chalcogens $(\mathrm{O}, \mathrm{S})$ have two six valence electrons, thus radicals like $\mathbf{R}-: \dot{\mathrm{S}}$ : when bound to graphene trend to reconstruct to ...-C-:S:-C-... with two saturated lone pairs, which are chemically inert, hence no further functionalization is possible with chalcogens. Therefore epoxygraphene and thioethergraphene are the only existing varieties of graphene functionalized compounds with chalcogen atoms.

Group 5 elements with 5 valence electrons can also bind to two graphene carbon atoms. In this case e.g. a nitrene radical R- $\mathbf{N}$ : after binding to graphene reconstructs to ...-C-Nं:-C-..., where unlike chalcogenes, the dangling bond (one unshared electron) is highly reactive and can bind to various radicals, the simplest of which is a hydrogen atom. This will form an aminographene.

Besides nitrogen in amines other heteroatom subsituents can be used for functionalization. Phosphinidene radical (R-P̈:) is a direct analog of nitrene, therefore everything said above about electron configuration and geometry of nitrene can be expanded to phosphinidene, while noticing that the increasing size of the $\mathrm{P}$ atom can produce more sterical congestions for the molecule. Carbene $(\mathbf{R}-\ddot{\mathbf{C}}-\mathbf{R})$ derivatives can bind to graphene in two ways: leaving a single lone pair or saturating it with hydrogen or bigger molecules. In the first case carbene functionalized graphene would be represented as ...C- $-\ddot{\mathbf{C}}-\mathbf{C} . .$. , in the second one, however, as ...C-RCR-C... The first case is similar to epoxy- and epthographene and is considered together with 
them, the second is considered along with other structures with the same moieties. An electron deficient boranylidene (R-B:) radical can also bind to a graphene sheet, in this case we may expect an upright geometry of the substituent due to absence of the lone pairs and weaker electrophilic properties.

Thus, there is an "epoxy"-like group of functioinalizing atoms (O, S, C), that cannot bind to anything else, and the $\dot{\mathrm{B}}, \mathrm{CH}_{2}, \dot{\mathrm{N}}, \dot{\mathrm{P}}$, etc. - based substituents, that have one unsaturated valence and thus can be attached to a hydrogen atom or to further more complex substituents like e.g. PFPA ${ }^{1}$.

In the next paragraph, band structure and conductivity studies of an epoxyfunctionalized graphene will be considered. The choice of the structure is dictated by its relative simplicity, along with didactic usefulness, and understanding of key concepts of covalent functionalization.

\subsection{Epoxy functionalization}

In this section we shall discuss the "epoxy"-functionalized graphene. Under "epoxy" we will consider all kinds of structures that appear by functionalization of a graphene sheet with chalcogenes and $\mathrm{C}$ atoms. Hereinafter they are labeled as $(\mathrm{O}, \mathrm{S}, \mathrm{C}, .)-$. epoxygraphene.

The structure of epoxygraphene was optimized first by the procedure from App. A.1. As a result of geometrical optimization a deplanarization of two carbon atoms occurred, adjoining the functionalizing chalcogene atom. This deplanarization is linked to the partial rehybridization of carbon atoms changing their electronic structure from pure $s p^{2}$ to $s p^{2}+s p^{3}$. No complete rehybridization occurs for these atoms because the angle of the $\mathrm{C}-\mathrm{C}$ bond still remains much less than $109^{\circ}$. A general $s p^{2+\eta}$ hy-

\footnotetext{
${ }^{1}$ Its particular applicability will be discussed later
} 


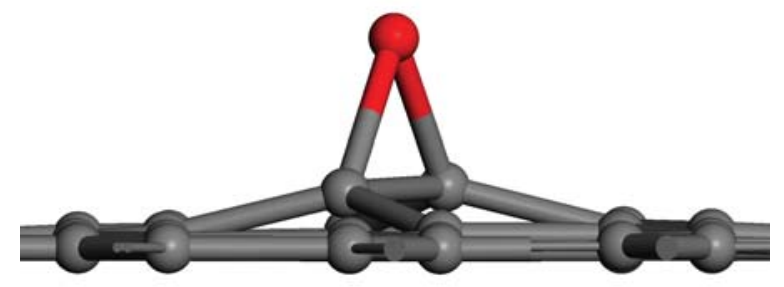

Figure 6.1: Fragment of the optimized structure of epoxy functionalized graphene (O-epoxygraphene). Carbon atoms are gray, oxygen -red.

bridization is expected to have a higher excitation energy than that of the symmetric $s p^{2}$ hybridization discussed before, because of the electron- electron repulsion which occurs in the hybridized orbital [114]. Comparing the bond length in C-,O-, and S-epoxygraphene, one can see that the shortest $\mathrm{C}-\mathrm{X}$ distance $(d(C-X))$ is observed when $\mathrm{X}=\mathrm{O}$, which is the most electronegative atom in the $\mathrm{C}, \mathrm{O}, \mathrm{S}$ row. Therefore $\mathrm{C}-\mathrm{O}$ bond is the strongest. However, due to its high electronegativity, donor properties of the oxygen atom are relatively weak compared to sulfur. Therefore the highest charge transfer occurs in S-epoxygraphene. This enhanced charge transport leads to the the strongest disturbance of the $\pi$-conjugated electron system of graphene for the given class of fuctionalizing compounds. Hence, additional electron donation manifests itself in stronger rehybridization and band gap opening in S-epoxygraphene. However, the degree of rehybridization $(\eta)$ is fairly low in these structures: the MO and WF pictures reveals that the states near the Fermi level are primarily constructed from graphene conjugated $\pi$ orbitals without significant admixture of the states from the heteroatom. (See Figs.6.3, 6.4) 


\subsubsection{Band structure of epoxygraphene.}

For the bandgap calculation we chose the configuration of one functionalizing atom per a 6x6 graphene supercell. Usually higher functionalization degrees lead to stronger discrepancy between the diagonal element of the Hamiltonian matrix (see Sec. 5.1.1 and discussion at the end of it.) As presented below, the band gaps are so narrow, that only a very excessive functionalization can create a band gap which is at least four times the thermal energy at room temperature. However, excessive functionalization with oxygen atoms (each honeycomb in a row functionalized) is known to cause "unzipping" and cracking of graphene sheets [133].

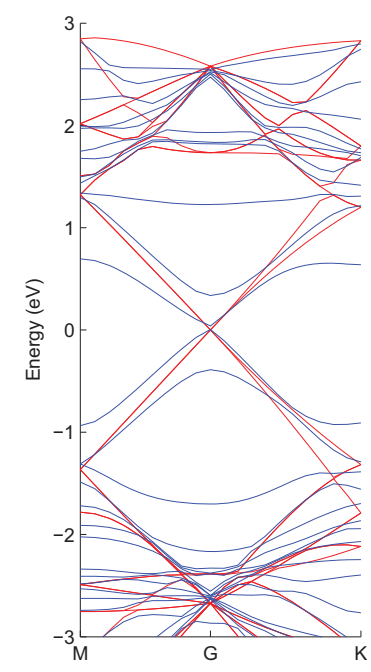

(a) O-epoxygraphene

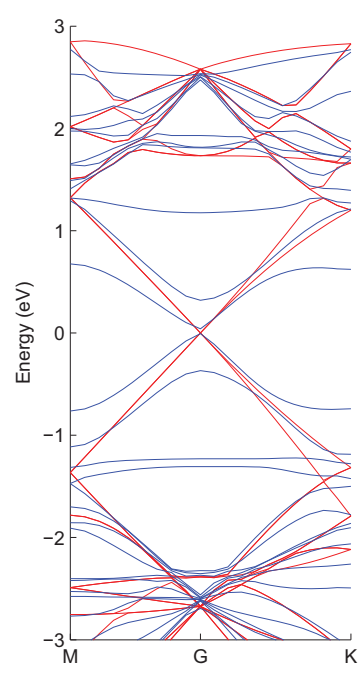

(b) S-epoxygraphene

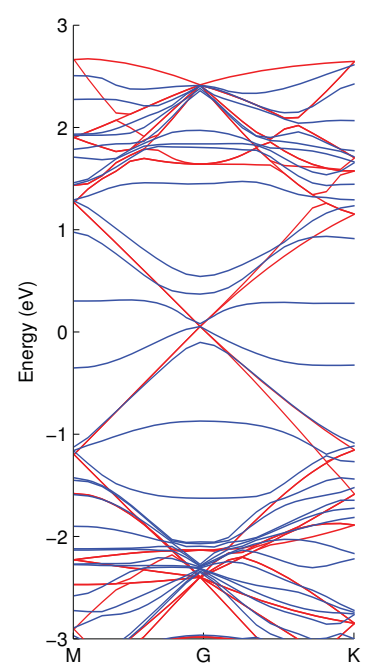

(c) C-epoxygraphene

Figure 6.2: Band structures of pristine (red) and epoxy-functionalized (blue) graphene. Band structures were aligned with respect of the position of the Fermi level

Numerical values of band gaps are summarized in Table 6.2 at the end of the chapter. Band gaps vary from $39 \mathrm{meV}$ for epoxygraphene to $41 \mathrm{meV}$ for epthiographene. Bandgaps increase as the charge transfer of the corresponding atom increases. Atomic 
Mulliken charges follow the same trend as band gaps do: -0.411e for epoxygraphene, -0.247 e for epthiographene, and -0.025 e for epcarbographene. The more electrons a heteroatom is able to donate or accept from the $\pi$-conjugated system (i.e. the larger the charge transfer), the more disturbance it causes in the conjugated system, and therefore, the higher is the difference between the diagonal elements of the Hamiltonian matrix. However, these bandgaps are still about the thermal energy at room temperature. Therefore epoxy- functionalization of graphene is most likely a dead end. But for didactic and benchmarking purposes, transport calculation for epoxygraphene are presented in the section below. 


\subsubsection{Wannier functions and transport in epoxygraphene.}

For the transport computations a fairly large (6x6) supercell is chosen, consisting of 85 atoms per unit cell, where the total number of bands considered in the nSCF calculation was set at $800^{2}$. SCF computation is conducted on a $331 \mathrm{MP}$ grid, nSCF - on 9 k-points in the whole BZ. The size of the supercell allowed not to conduct separate computation of the semi-infinite electrodes, but rather to assume that the Wannier functions far away (about 3 honeycombs) from the oxygen atom are not disturbed by functionalization and thus can be taken as the electrodes' Wannier functions. An advantage of this scheme is that the Fermi levels in the conductor region and the electrodes are matched automatically. Also, since in the experiment graphene nanoribbons are often on the order of $1 \mu \mathrm{m}$ in width, we may assume bulk conductivity mode for all structures under consideration.

Trial Wannier functions were initialized as two subsets: graphene subset is initialized as a set of $\sigma$-type orbitals located between carbon atoms and dangling $p_{z}$ orbitals. The subsets of heteroatoms are explicitly defined as their atomic orbital set $\left(1 \mathrm{~s}+3 \mathrm{p}\right.$ orbitals). Binding to the graphene sheet is achieved by mixing of $p_{z}$ orbitals of carbon atoms adjoined to the heteroatom and its $s$ AO (Fig. 6.3). Other AOs of the heteroatom $\left(p_{x}, p_{y}, p_{z}\right)$ do not participate in the bonding process: $p_{x}$ and $p_{y}$ remain undistorted and only slight interaction is observed with $\pi$-system of graphene (Fig. 6.4(a)), $p_{z}$ does not participate at the bonding process forming the lone pair (Fig. 6.4(b)).

The WF picture confirms very weak interaction between the heteratoms and the electron system of graphene. The HOMO consists entirely of graphene $\pi$ orbitals, whereas the doubly degenerate LUMO of $p_{x}$ and $p_{y}$ orbitals of the heteroatom. Ab-

\footnotetext{
${ }^{2}$ This number is obtained from the rule of thumb that for good convergence the number of empty band considered should be three times of the number of the occupied ones
} 


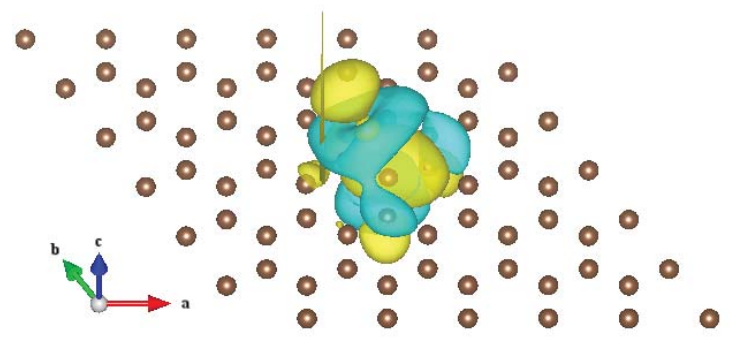

(a) $p_{z}$

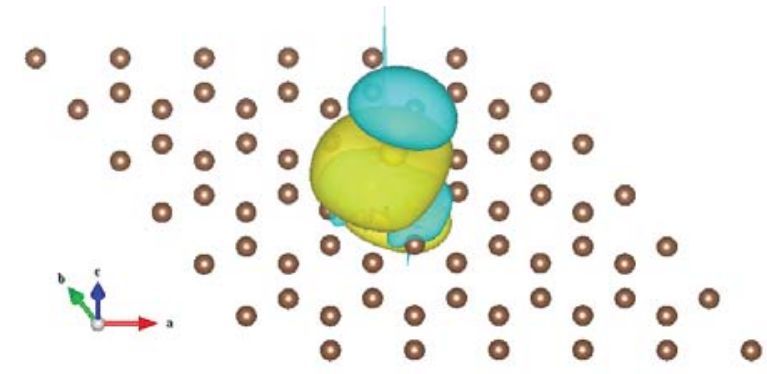

(b) $s$

Figure 6.3: Wannier functions of S-epoxygraphene. Binding set

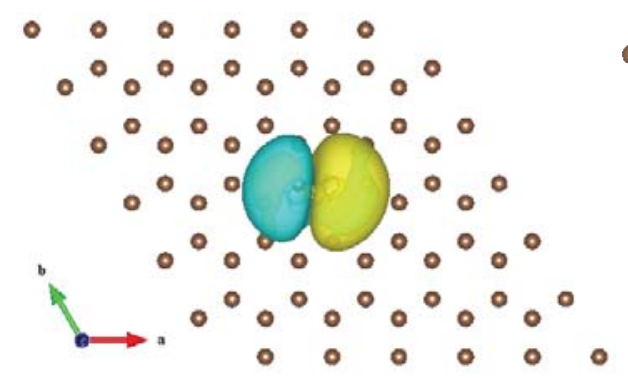

(a) $p_{x}$ or $p_{y}$

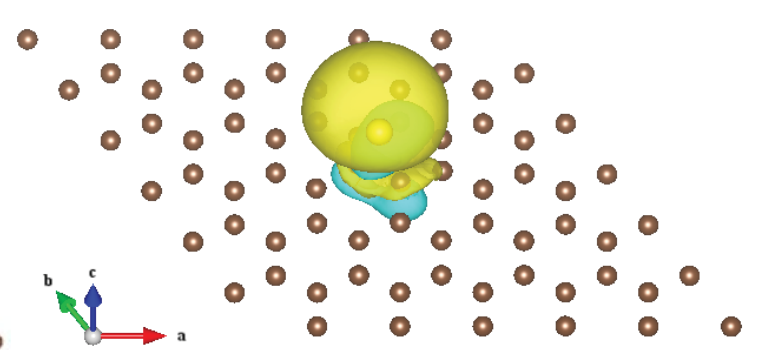

(b) $p_{z}$

Figure 6.4: Wannier functions of S-epoxygraphene. Nonbinding set 
sence of mixing allows to represent the Hamiltonian matrix of the system is the block diagonal form, so that the eigenvector problem almost splits into two independent subsets of secular equations. Therefore, the diagonal elements of the graphene block of the Hamiltonian matrix are almost undisturbed by epoxy-funtionalization. This fact also manifests itself in the transport properties: conductance of epoxygraphene (Fig. 6.5) closely resembles that of pristine graphene, and differ from that only due to presence of additional states imposed by the heteroatom and folding of the BZ.

As is can be seen from Fig. 6.5, the highest conductivity occurs in S-epoxygraphene. The trend in conductance (see Fig. 6.5 and table 6.2) follow the same trend with the trend in the band gap. The reason for that is that since epoxygraphene has very little mixing of AOs, its band structure can be split into "graphene bands" and "heteroatom bands", which would bring the Hamiltonian matrix to the block-diagonal shape. Since the determinant of a block-diagonal matrix is a product of the determinant of the corresponding blocks, conductance calculated from that matrix can be treated separately as well. Also because of the very little mixing, the heteroatom energy levels possess very small dispersion, and thus the electrons, occupying these bands are extremely heavy and do not contribute much to conductance. This analysis is very important for further consideration, especially regarding flat bands appearing in different functionalization schemes.

Almost all structures still preserve graphene like V-shaped conductance behavior in the vicinity of the Dirac-point. They are disturbed by the presence of heteroatom levels which despite their small dispersion make some contribution to conductance. The higher the dispersion of these bands, i.e. the higher is the degree of functionalization, the higher is the contribution to conductance. That explains the similarity in the band gaps and conductance trends.

That being said, one concludes that the key to band-gap opening and high con- 
ductance is such sort of functionalization is the degree of hybridization, i.e. mixing between graphene MOs and MOs of the functionalizing groups. Attempting to accommodate extra electrons donated by the heteroatom, graphene MOs and MOs of the fictionalizing groups will hybridize strongly creating more low energy collective MOs. The next step towards enhancement of charge transfer is to bind the heteroatom with another electron donor (acceptor). Several examples of this are discussed in the the rest of chapter VI and in chapter VII. 

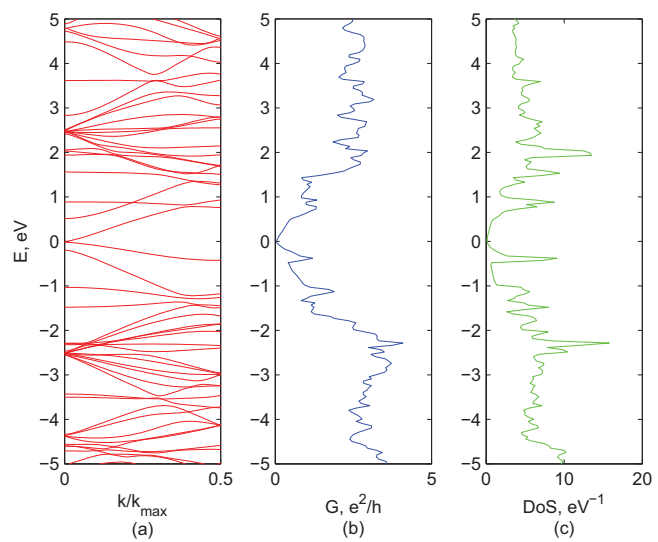

(a) C-epoxy
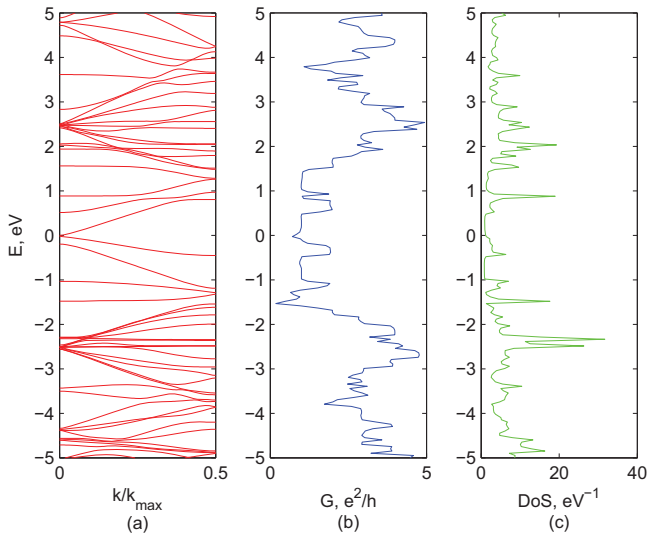

(b) C-epoxy
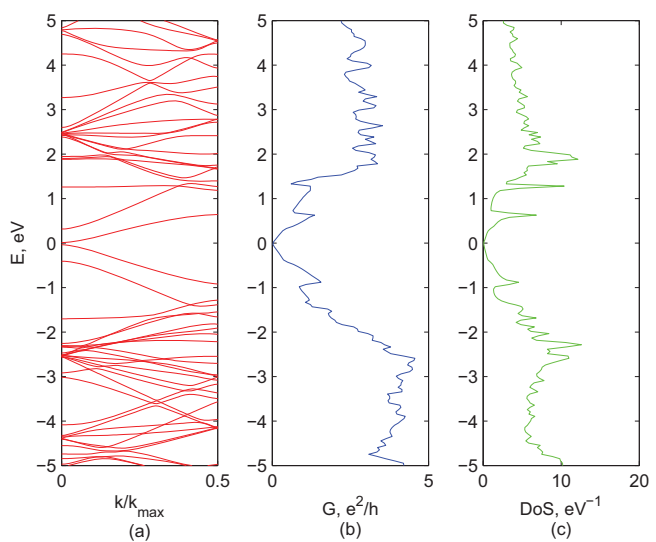

(c) O-epoxy
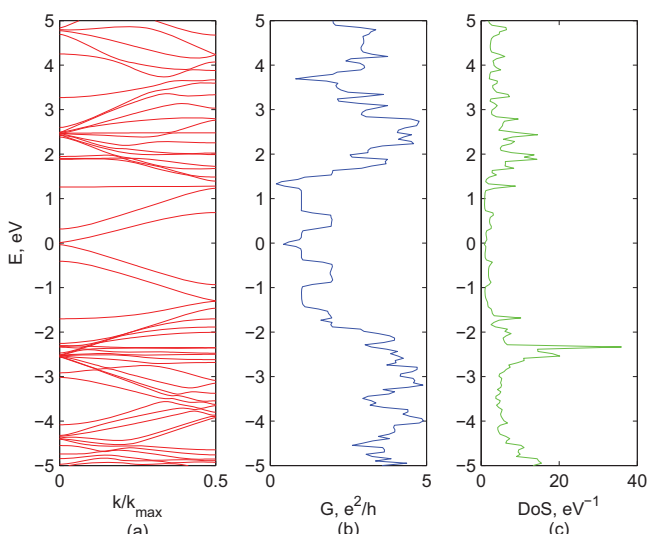

(d) O-epoxy
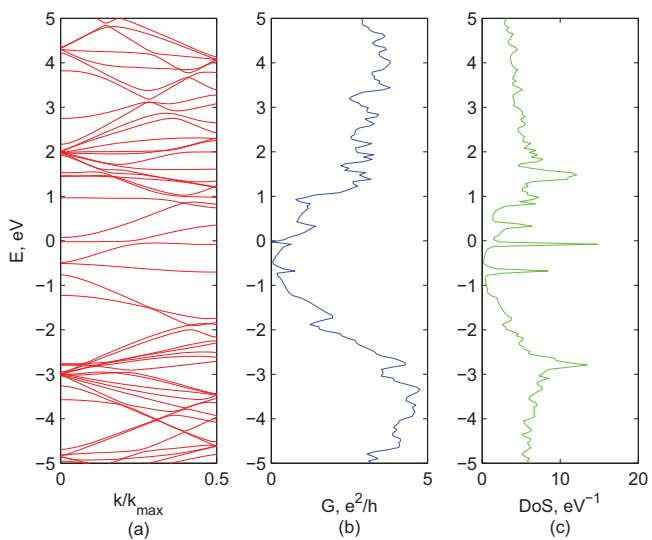

(e) S-epoxy
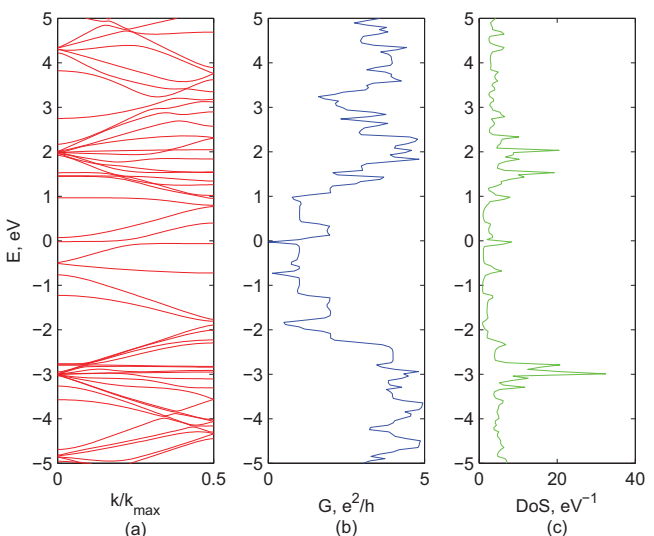

(f) S-epoxy

Figure 6.5: Band structure (a), conduction (b), and density of states (c) of epoxygraphene in the $\Gamma-\mathrm{K}$ (top row) and $\Gamma-\mathrm{M}$ (bottom row) directions. 


\subsection{Amino functionalization}

Atoms with odd number of valence electrons (B,N,P,etc.) after bonding to sheet still have one dangling bond that can be terminated with hydrogen. This gives rise to a family of "amino"-functionalized graphene. Again, as in the previous paragraph under "amino" we consider a group of radicals, : $\ddot{\mathrm{NH}},: \ddot{\mathrm{PH}},: \mathrm{BH},: \mathrm{CH}_{2},: \mathrm{SiH}_{2}$ that use one electron pair to bind to graphene sheet, and hereinafter we refer to their corresponding compounds as $\mathrm{N}$-aminographene, P-aminographene, B-aminographene, etc.

The structure of aminographenes was optimized starting from the structure of epoxygraphene with one $\mathrm{H}$-atom initially placed right above the heteroatom. As the result of optimization the position of the hydrogen atom differed for different electronic structure of the heteroatom. If a heteroatom has a lone pair, like $\mathrm{N}$ or $\mathrm{P}$ than the angle $\angle$ (H-X-cent), where "cent" stands for the center of the $\mathrm{C}$-C bond to which the heteroatom is attached, decreases from the initial straight-upright configuration, $180^{\circ}$ (See Fig. 6.6), to almost $90^{\circ}$, depending on which $\mathrm{AO}$ forms the lone pair (e.g. $1 \mathrm{p}$ for for nitrogen and $2 \mathrm{p}$ for phosphor).

Angle $\angle$ (H-X-cent) declines from $180^{\circ}$, which correspond to $s p^{3}$ hybridization of the boron atom, to almost $90^{\circ}$ for $\mathrm{P}$-aminographene, since lone pair acts an additional ligand essentially changing the hybridization from $s p^{3}$ to $s p^{2+\eta}$. The rehybridization parameter $(\eta)$ increases from $\mathrm{N}$ to $\mathrm{P}$ as the principal quantum number of valence electrons increases. Tetrahedral angles for C- and Si- derivatives, are very similar and slightly higher than the ideal tetrahedral angle $\left(109^{\circ}\right)$, since the bonding environment "on the top" and "on the bottom" is not symmetric, and graphene carbon atoms also have residual binding between each other due to not complete rehybridization from $s p^{2}$ to $s p^{3}$. Bond lengths in Figure 6.6 reveal a trend with depression, which is 


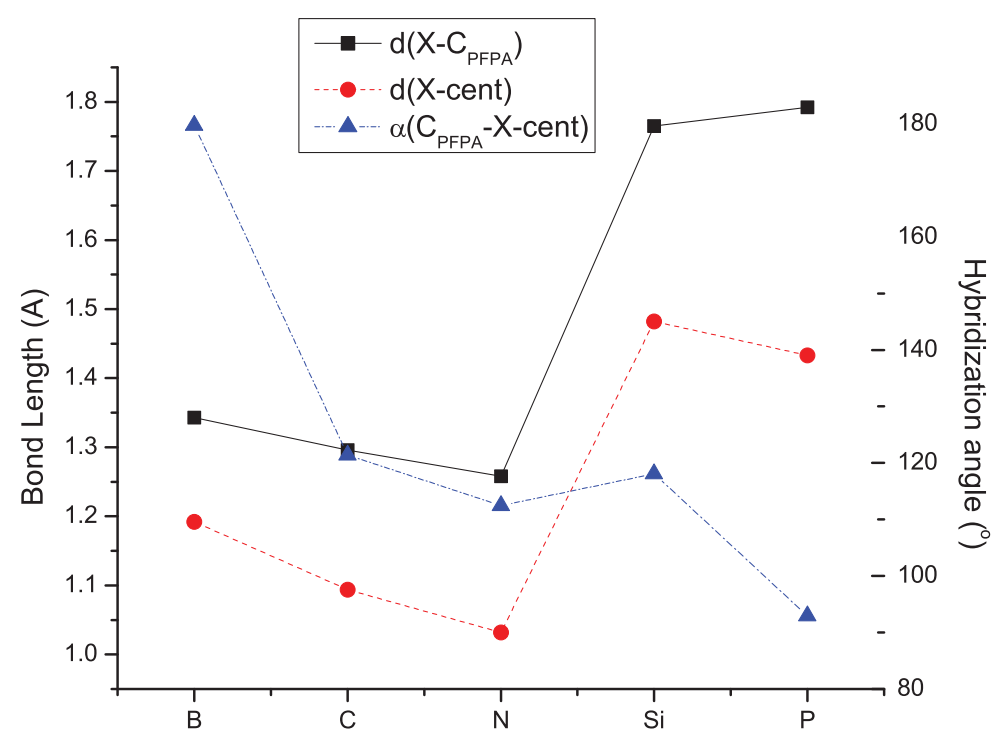

Figure 6.6: Bond lengths and hybridization angles in aminographene.

associated with the nitrogen atom since it is the most electronegative element in the B-C-N row. As the size of the heteroatom drastically increase, i.e. by moving from the second period to the third (Si-P), bond lengths undergo a jump, but continue to follow a declining trend.

\subsubsection{Band structure of aminographene.}

Band structures of aminographene resemble that of the epoxy structures (smallest band gap occurs in the $\Gamma$ point due to BZ folding). Band structures are shown in Fig. 6.7, and numerical values of band gaps are summarize in Table 6.2 at the end of the chapter.

Band gaps extracted from Fig. 6.7 are fairly narrow. Comparing these band gaps with thermal energy at room temperature $\left(300^{\circ} \mathrm{K} \approx 25 \mathrm{meV}\right)$, we conclude that amino 


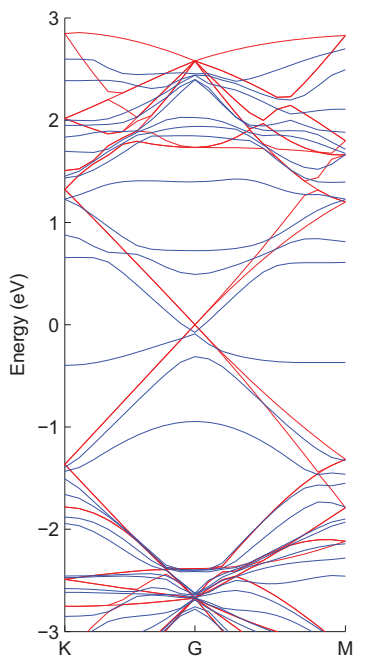

(a) B-aminographene

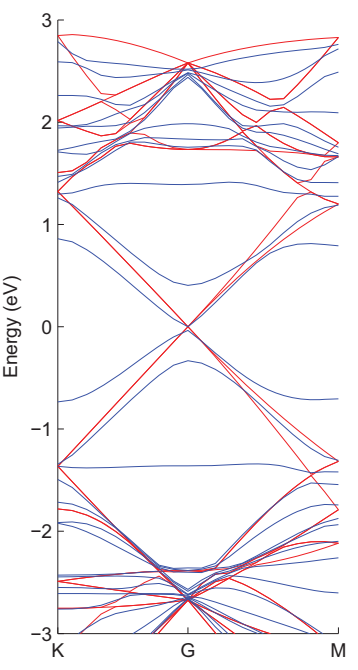

(b) C-aminographene

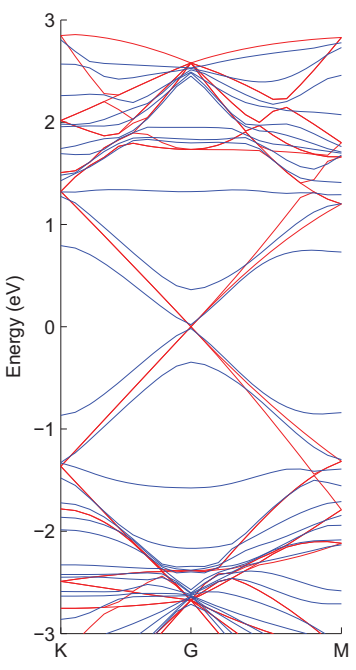

(c) N-aminographene

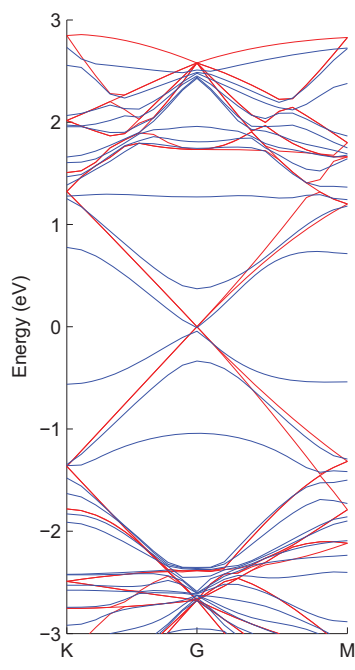

(d) P-aminographene

Figure 6.7: Band structures of pristine (red) and amino-functionalized (blue) graphene. Band structures are aligned with respect to the position of the Fermi level

functionalization is hardly of any practical use ${ }^{3}$. The reason for such low impact on the $\pi$-conjugated structure of graphene is because a heteroatom bonded to a single hydrogen atom cannot accommodate a lot of extra electron density from graphene. This is because hydrogen with only one valence electron and only one hole is neither an efficient donor, nor an efficient acceptor, and it makes the "amino" group a weak functionalizer as well.

In order to enhance acceptor properties we need to attach a substituent to the heteroatom that can spread enough charge to reduce excessive negative charge of the heteroatom. One of such efficient substituents is a phenyl ring because it also possesses $\pi$-conjugated structure as well. This phenyl functionalization gives rise to the so called PFPA-FG graphene.

\footnotetext{
${ }^{3}$ Except maybe of infrared bolometers, that are operated under cryogenic temperatures.
} 


\subsection{PFPA functionalization}

PFPA (perfluorophenylazide) is a benzene molecule with two substituents in the para configuration: azide and possibly another functionalizing group, like methyl, or a C-Si-C chain. (Fig. 6.8)
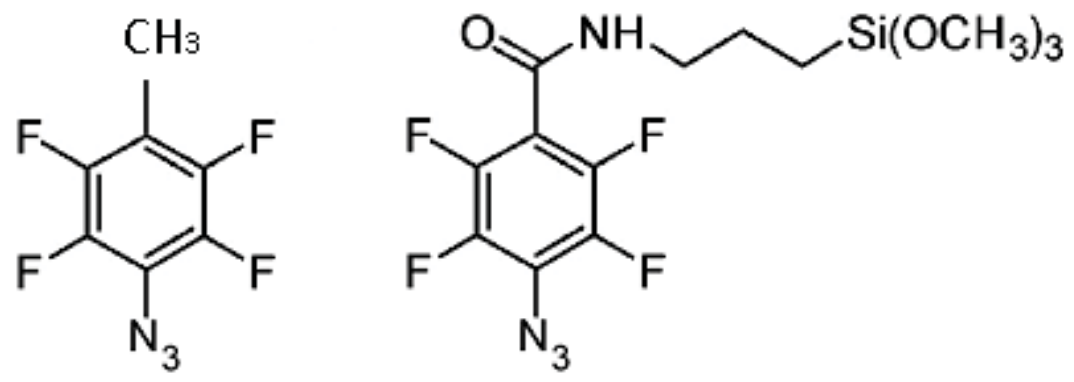

Figure 6.8: Perfluorophenylazide molecule with methyl and "silane" moieties.

PFPA has drawn widespread interest for functionalizing graphene because of its relatively high reactivity along with its high affinity to graphene's carbons (by the first para substituent). It also has the ability to immobilize graphene to silicon wafers (via second para substitution) (Fig. 6.9) and modify its band structure [123, 134, 135].

In order to calculate any structural or electronic properties one first needs to obtain an optimized geometry for both bare PFPA and PFPA functionalized graphene (PFPA-FG). A straightforward geometrical optimization of the atomic models fails due to a very high number of unconstrained variables, therefore we have employed the following scheme. Starting from epoxygraphene, we replace the oxygen atom by an amino group, which in turn, is then replaced by an azide group. The two nitrogens from the azide group are then replaced by a benzene ring, and finally, hydrogen atoms in the benzene ring are replaced by $\mathrm{F}$ or $\mathrm{CH}_{3}$. We ignore the "silane" tail since it will not affect any electronic properties of PFPA-FG being uncoupled from 


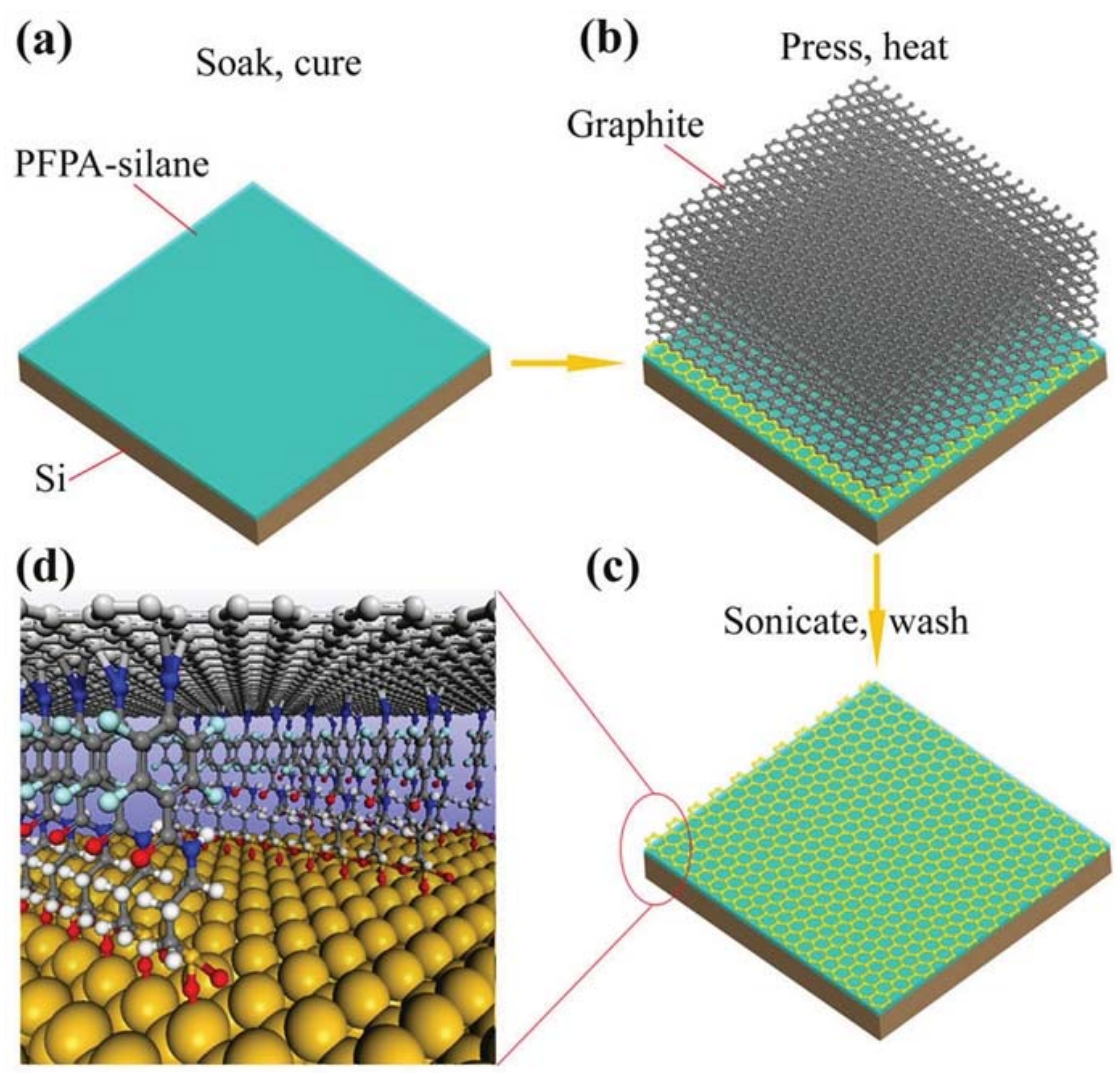

Figure 6.9: Attachment of a PFPA molecule to graphene and to a silicon substrate Adopted from [134]

the functionalizing "hot-spot". Geometrical optimization is performed on each step of the procedure,yielding very close final atomic positions at each step. Optimized PFPA-FG exhibits same type of bonding to graphene as aminographene.

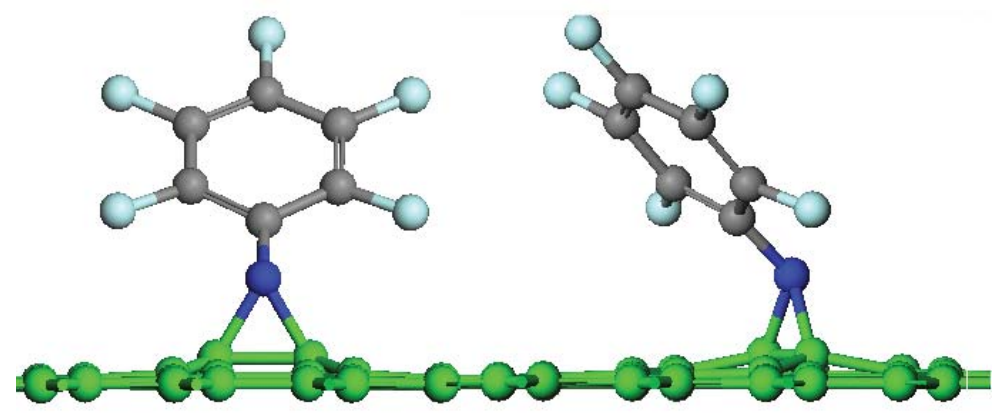

Figure 6.10: Structure of N-PFPA functionalized graphene. 


\subsubsection{Reaction and bonding between PFPA and graphene.}

High reactivity of azides is dictated by the weakness of the second $\mathrm{N}-\mathrm{N}$ bond in the azide tail. While bonding, a PFPA molecule splits off a $\mathrm{N}_{2}$ molecule, turning into a nitrene radical, which in turn, has high electronegativity (since it is an electrophil, acceptor) and easily binds to nucleophilic sites in graphene.

Quantitative description of electrophilicity and nucleophilicity can be obtained from the Fukui function [136]. The Fukui function is defined as a functional derivative over the number of electrons $N=\int \rho(\mathbf{r}) d \mathbf{r}$ :

$$
f(r)^{\alpha}=\left(\frac{\partial \rho(\mathbf{r})}{\partial N}\right)^{\alpha}
$$

where the superscript $\alpha= \pm$, indicate whether the derivative is evaluated at $N \pm$ $\delta, \delta \longrightarrow 0$. The function $f^{+}(r)$ is associated with the lowest unoccupied molecular orbital LUMO and measures reactivity toward a donor reagent (like graphene), the function $f^{-}(r)$ - with HOMO and measures reactivity toward an acceptor (like nitrene) reagent.

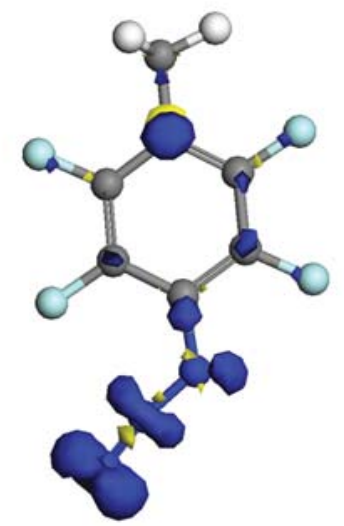

(a)

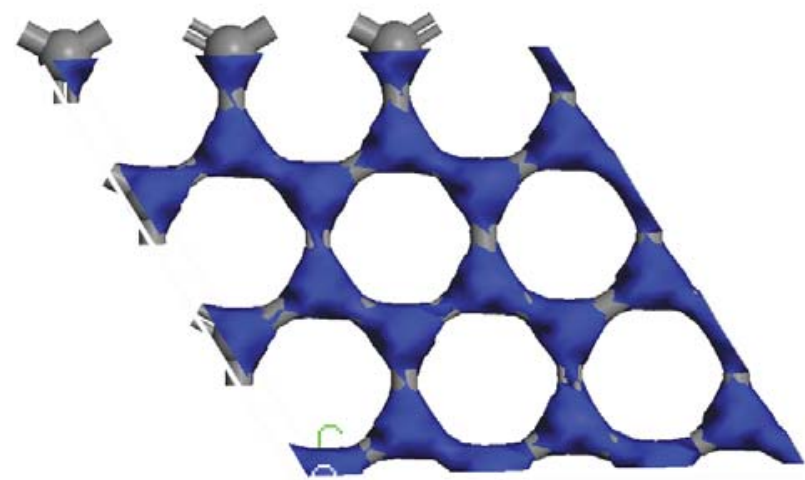

(b)

Figure 6.11: Fukui functions: $f^{+}$for a PFPA molecule (a), $f^{-}$for graphene (b). 
From Fig. 6.11 it can be seen that one of the "hot spots" of the PFPA molecule is the nitrogen atom, bound to the phenyl ring. In this configuration nitrogen has four unshared electrons distributed over two lone pairs. Two of these four electrons form two bonds with carbon atoms of graphene, and the remaining two stay attached to the nitrogen atom as a lone pair. This electron configuration explains optimized geometric structure of PFPA-FG, namely the bending of the PFPA molecule with respect to the graphene plane: the nitrogen would prefer trigonal coordination with one of the apexes to the the lone pair (Fig. 6.10). The same can be observed for the phosphenidene radical, however due an increased atomic volume and the volume of the lone pair, deviation from the upright geometry will be even more significant. The atomic structure of C-PFPA (as well as Si-PFPA), in contrast suggests that there should be two phenyl rings attached the the carbon "heteroatom" (Fig. 6.12).
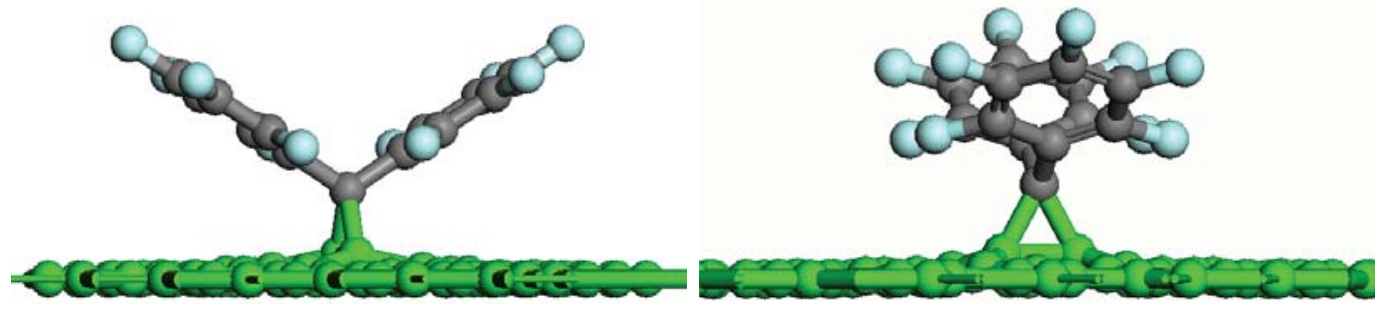

Figure 6.12: Structure of C-PFPA functionalized graphene.

Geometrical parameters that characterize PFPA-FG structures are the angle $\angle$ (C$\mathrm{X}$-cent) and $\mathrm{d}(\mathrm{X}$-cent $)$. $\mathrm{C}$ denotes the carbon atom of the phenyl ring bound to a heteroatom, "cent" - center between two carbons of graphene sheet to which the heteroatom (X) binds. Bonding angles between graphene and heretoatoms decrease almost linearly in the B-N-C-P-As row (Fig. 6.13 right scale). Bond lengths $d(C-X)$ in Fig. 6.13, similar to Fig. 6.6, reveal a trend with depression (Fig. 6.13 left scale), which is associated with the nitrogen atom since nitrogen is the most electronegative atom in the B-C-N row. The explanation for this trend is very similar to the amino 
case and is not repeated here. However, noticing that $\mathrm{C}_{P F P A} \mathrm{X}$ bond length in PFPA-FG are on average $29 \%$ longer than in amino-FG, and X-cent are about $2 \%$ longer than in corresponding amino structures, i.e. on average bonding to PFPA is weaker than in the amino case. Despite that charge transfer in PFPA-FG structures is slightly $(\sim 5 \%)$ higher than in amino-FG. It is enough to open a six times wider gap (in case of $\mathrm{N}$ - the most efficient covalent functionalizer) in PFPA-FG structures compared to amino-FG. To pursue a detailed explanation of this phenomenon we compare electronic structures of functionalizers of both types.

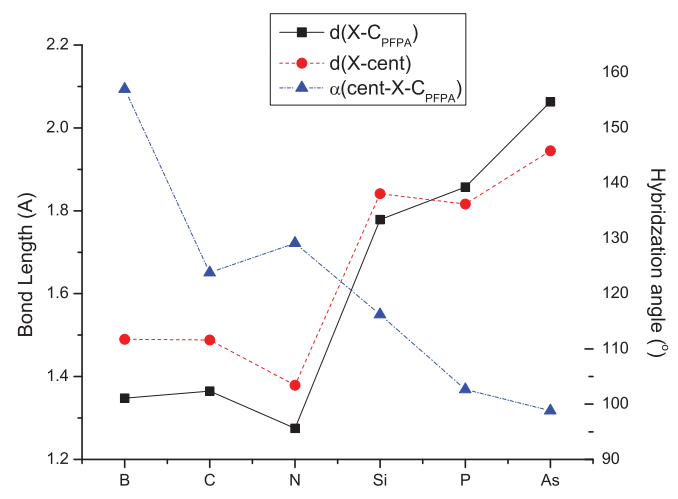

Figure 6.13: Bond lengths and hybridization angles in PFPA-FG 


\subsubsection{Band structure of PFPA-FG}

Acceptor properties increase in the row R-B:, R-P̈:, R- $\ddot{\mathrm{N}}$; and thus we can expect that band gap will follow the same trend, since the width of the gap is proportional to the charge transfer, which increases as electronegativity decreases. Both trends are proportional to the difference between the diagonal elements of the Hamiltonian matrix. A detailed description of that phenomenon resembles that for epoxy- and aminographene, and thus is not repeated here.

Band gaps are summarized at the end of the chapter in Table 6.2. It can be seen that the widest gap that can be induced in graphene by functionalization with a single PFPA molecule per 6x6 graphene supercell is $0.24 \mathrm{eV}$. Functionalization with two molecules slightly increases the gap to $0.28 \mathrm{eV}$. This is more than 100 times higher than the thermal energy at room temperature and thus can find industrial application in microelectronics, providing a feasible ON/OFF ratio for transistors.

Band structure of PFPA-FG resembles that of aminographene. Previously intersecting $\pi$ and $\pi^{*}$ bands that formed the Dirac cones are now pulled apart by the underlying $\sigma$ bands, arising from the interaction of the heteroatom with the system of grapheneś $p_{z}$ orbitals. This $\sigma-\pi$ interaction causes rehybridization of the carbon atoms from the $s p^{2}$ to $s p^{2+\eta}$ state. The degree of additional hybridization $(\eta)$ is directly related to the band gap, as it was discussed above for epoxygraphene. Unlike the case of haptic functionalization discussed below, $s p^{2} \longleftarrow s p^{3}$ rehybridization causes deplanation of graphene structure. In this case degeneracy, that was previously imposed by symmetry on phonon modes, is now removed, and thus the electron phonon scattering is significantly stronger in deplanated structures than is bulk- and/or rigid structures.

Comparing the corresponding band gaps of PFPA- and amino-graphene, one finds 


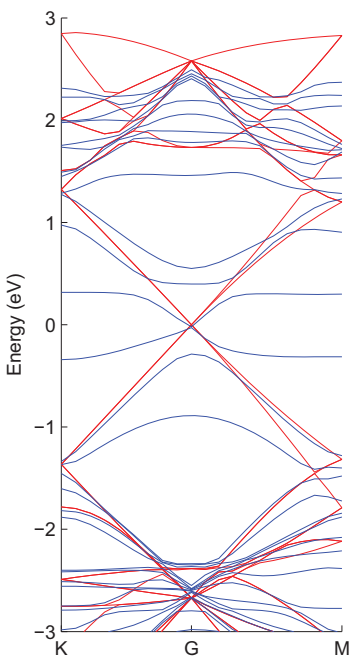

(a) B-PFPAgraphene

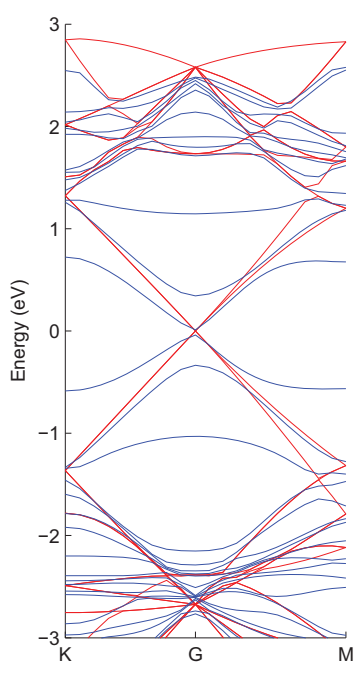

(d) Si-PFPAgraphene

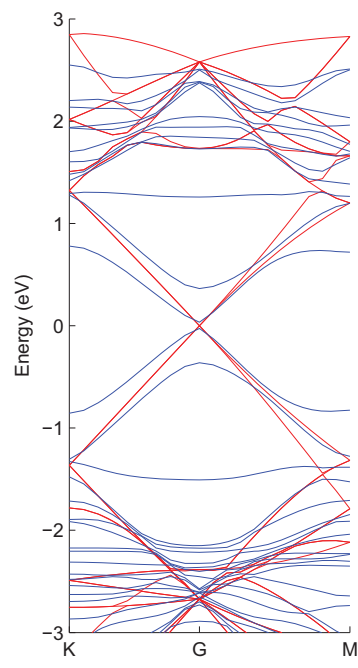

(b) C-PFPAgraphene

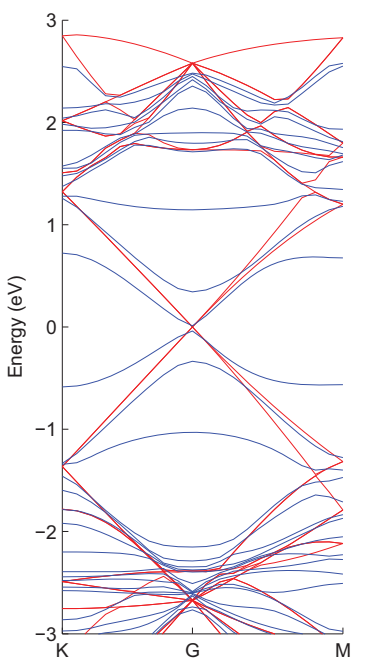

(e) P-PFPAgraphene

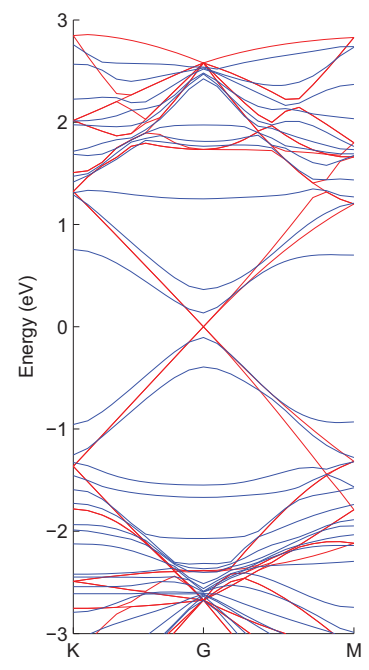

(c) N-PFPAgraphene

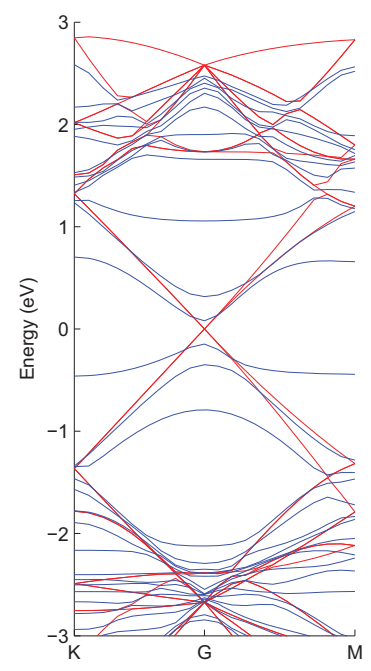

(f) As-PFPAgraphene

Figure 6.14: Band structures of pristine (red) and PFPA-functionalized (blue) graphene. Band structures were aligned with respect of the position of the Fermi level.

that in all cases the gap in PFPA-graphene is larger than in aminographene. The reason for this is probably the ability of a phenyl ring to accommodate a higher electron density, that leads to an increase of the charge transfer through the heteroatom and 
thus enhances charge transfer from graphene sheets to the functionalizing molecules.

Table 6.1: Charge, charge transfer $(\Delta Q)$, and relative electronegativity $\left(\chi_{X} / \chi_{C}\right)$ in PFPA-FG.

\begin{tabular}{cccccc}
\hline Compound & $\mathrm{X}$ & $\mathrm{C}(\mathrm{up})$ & $\mathrm{C}($ down $)$ & $\Delta Q$ & $\chi_{X} / \chi_{C}$ \\
\hline B-PFPA & +0.608 & -0.151 & $-0.251 /-0.256$ & +0.406 & 0.80 \\
C-PFPA & -0.058 & -0.078 & $-0.075 /-0.075$ & -0.054 & 1.00 \\
N-PFPA & -0.277 & +0.103 & $-0.043 /-0.045$ & -0.314 & 1.19 \\
P-PFPA & +0.377 & -0.431 & $-0.259 /-0.253$ & +0.398 & 0.86 \\
As-PFPA & +0.018 & -0.315 & $-0.186 /-0.174$ & +0.087 & 0.85 \\
\hline
\end{tabular}

$\mathrm{C}($ up) stands for the carbon atom from the phenyl ring, linked with the heteroatom, $\mathrm{C}$ (down) stands for two carbon atoms from the graphene sheet, linked with the heteroatom.

Charge transfer is calculated in the same way as in sec. 6.3.1. Table 6.1 demonstrates that the highest charge transfer occurs in N-compounds. Considering the trends in the band gap, the sign of the $\Delta Q$ indicates donor or acceptor properties. Charge transfer in turn, depends on the relative (with respect to carbon) electronegativity of the heteroatom. Strong donors (small relative electronegativity) result in small band gaps (B, P), whereas strong acceptors (high relative electronegativity) result in wider band gaps. Weak donors and acceptors, such as arsenic and carbon result in intermediate values of the band gap. Therefore the N-PFPA compound probably possesses the widest band gap among all possible PFPA-FG compounds. Based on this fact we will focus our transport computations on the transport properties of N-PFPA-FG. 


\subsubsection{Wannier functions and transport in PFPA-FG}

Since PFPA-functionalization leads to opening of a significant gap in the electron dispersion spectrum of functionalized graphene, the curvature of the top valence and bottom conduction bands at the $\Gamma$ point now leads to a finite electron mass. Therefore we cannot expect electron mobility to be as high as in pristine graphene.

To understand the nature of mobility degradation caused by functionalization using the NEGF method in localized Wannier functions representation. For transport calculations can following models a N-PFPA supercell can be considered:

- a relatively narrow GNR with a PFPA molecule connected to pristine GNRs on both sides (Fig. 6.15a). The blocks of pristine GNR play the role of infinite 1D electrodes, that due to the shift of their energy spectrum by the value of their self-energies ( $\Sigma_{L, R}$, see Eq. 3.18), create unmatching states into which electrons, otherwise traveling ballistically, can scatter.

- an interface of PFPA-FG sandwiched between two semi-infinite graphene sheets (Fig. 6.15b). The difference from the previous case is absence of spatial confinement in the lateral direction, and thus absence of the boundary states which otherwise would cause additional scattering.

- an infinite sheet with one functionalizing molecule per 6x6 graphene supercell (Fig. 6.10). In this geometry no boundary states are created and thus the whole scattering is attributed to Coulomb scattering off electron density disturbances and electron-phonon scattering.

Only the last case is relevant to the practical application, since GNRs conventionally produced are usually of the size $\approx 10 \mu \mathrm{m}$ wide ${ }^{4}$. This corresponds to $\approx 10^{4}$

\footnotetext{
${ }^{4}$ Narrower GNRs can be produced by unzipping CNTs $[133,137]$
} 


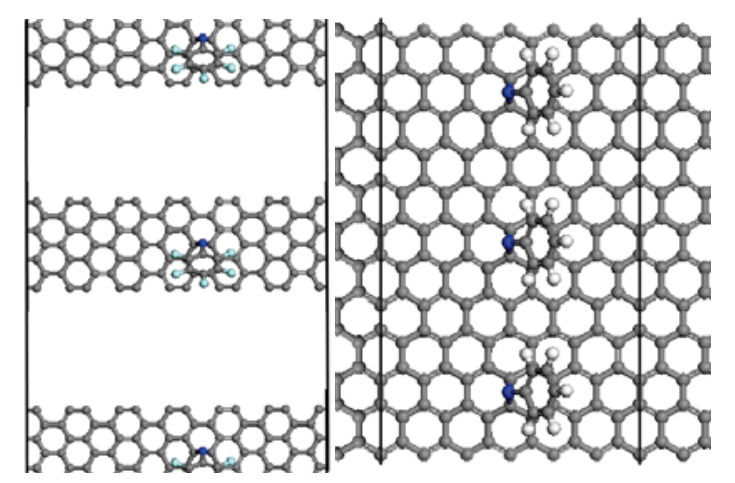

(a)

(b)

Figure 6.15: (a) GNR with a single PFPA molecule (bold lines indicate cell edges in lateral direction), (b) Interface layer of PFPA-FG sandwiched between pristine graphene sheets

honeycombs. At this number of honeycombs BZ folding leads to an almost infinite increase of bands in a very small reciprocal cell, and thus periodic boundary conditions can be imposed in both directions. Also this would allow one to eliminate the effects of boundary scattering and and focus on effects of functionalization on electrical conductivity.

Wannier functions of PFPA-FG. A trial set of Wannier functions in PFPAFG structures was initialized in the similar way as described in section 6.2.2. The graphene subset was initialized as a set of $\sigma$-type orbitals located between carbon atoms and nonbonding $p_{z}$ orbitals. The PFPA subset was explicitly defined as a full atomic orbital set $(1 \mathrm{~s}+3 \mathrm{p}$ orbitals $)$ for the nitrogen atom. The phenyl ring subset is initialized in the same way as graphene: six $\sigma$-type orbitals located between carbon atoms, five $\sigma$-type orbitals describing $\mathrm{C}$-H bonds, one $\sigma$-type orbitals representing C-N bond, and six dangling $p_{z}$ orbitals, centered on carbon atoms.

The electronic structure of carbon atoms belonging to the graphene subset is, as expected, distorted by functionalization (Fig. 6.16(a)) relative to the atoms lo- 


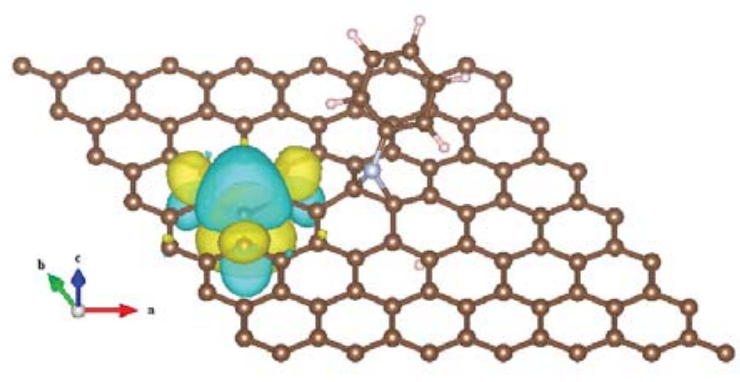

(a)

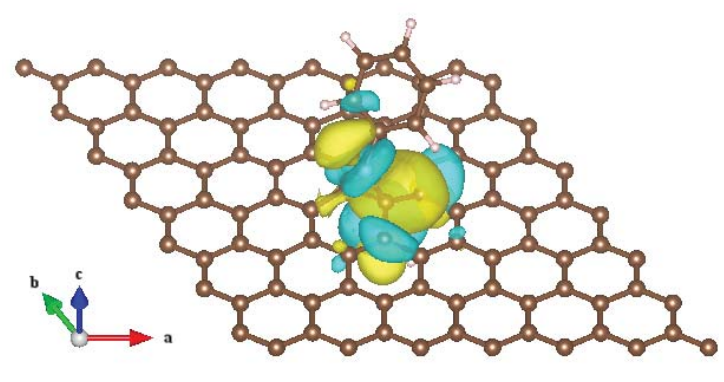

(b)

Figure 6.16: Wannier functions of carbon atoms in N-PFPA-FG. Far away from the functionalizing molecule (a) and in the direct vicinity to it (b)

cated further away from the functionalizing molecule (Fig. 6.16(b)). This distortion is responsible for the alteration of the band structure and transport properties in functionalized graphene.

Heteroatom $(\mathrm{N})$ interacts with the $\pi$-conjugated electron system of graphene with its $s$ and $p_{z}, p_{x}$ and $p_{y}$ orbitals contributing more towards the lone pair and even though it implicitly affects graphene $\pi$-structure, its contribution is not significant compared to $s$ and $p_{z}$ (Fig. 6.17).

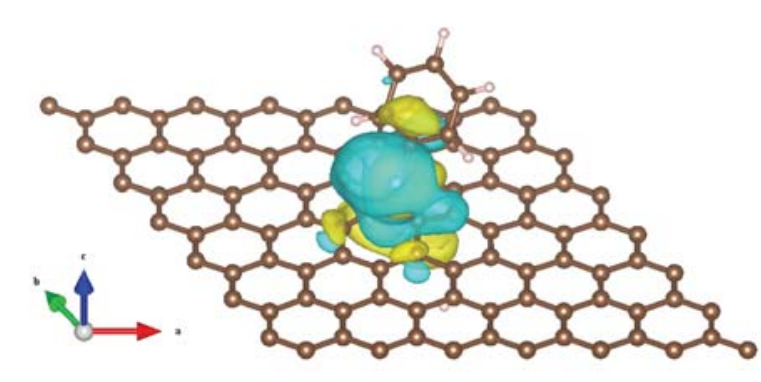

(a) $\mathrm{s}$

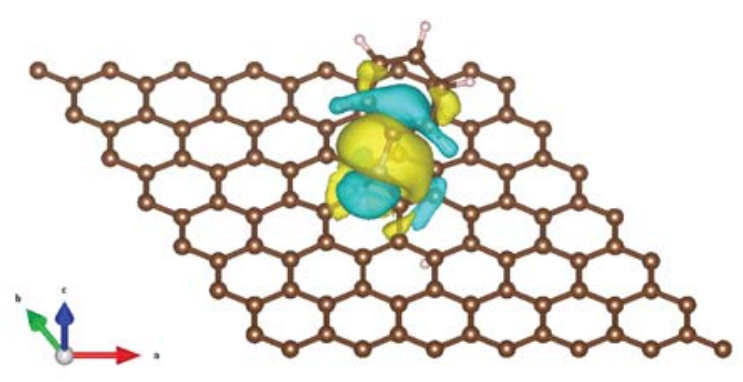

(b) $p_{z}$

Figure 6.17: Wannier functions of the heteroatom in N-PFPA-FG.

Electric conductivity of PFPA-FG. The set of Wannier functions obtained in the previous section effectively describes the conductivity in energy range $\pm 5 \mathrm{eV}$ Con- 
ductivity vs bias curves in Fig. 6.18, exhibit very similar behavior to the transport curves in pristine graphene. (Fig. 5.11 and Fig. 5.12).

The most pronounced peculiarity of the 1D band structure-conductivity-density of states (BCD) curves in Fig. 6.18 is the typical conductivity behavior in the vicinity of the former Dirac point demonstrating the same feature (Fig. 6.21(a)) as that observed in epoxy-graphene. As before functionalization creates extra bands in the band structure of functionalized graphene bands. This bands come from molecular levels of the adducts. Depending on the degree of hybridization of those levels with the electronic structure of graphene, they may have different dispersion. In particular, B-, As-, and Si-PFPA-FG creates a flat band in the vicinity of the Fermi level, that does not contribute to the conductivity of these compounds. The N-, P-, and CPFPA-FG, in turn do not possess those flat bands in their band structure and mainly preserve Dirac cones in their band structure. This has direct influence on conductivity of PFPA-FG compounds. In Fig. 6.19 we summarize our findings about electric conductivity of PFPA-FG. In order to compare scattered results for conductivity calculation presented in this chapter, we plot conductivity as a function of the atomic number of the binding heteroatom. This arrangement may seem not the best one, since all heteroatoms, considered for PFPA functionalization fall intro three clusters of neighboring groups. These can be arranged in different ways: by rows B-C-N Si-P — As, or by periods: B - C-Si — N-P-As, however, disregarding which of the possible arrangements is chosen, it can clearly be seen that conductivity follows an ascending trend with saturation.

Highest average conductivity is predicted for As-PFPA-FG, however, the bandgaps (Table 6.2) are in inverse relation with conductivity. Therefore practical application of PFPA-FG is a trade off between high ON/OFF ratio, supplied by broad (relative to kT) band gap and electron mobility. Si and $\mathrm{C}$ derivatives clearly do no follow the 

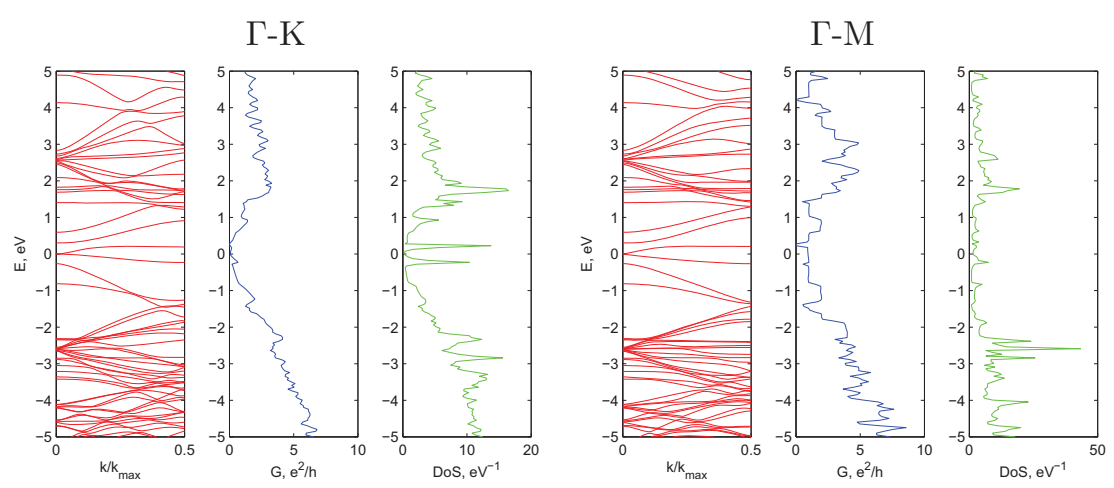

a) B-PFPA
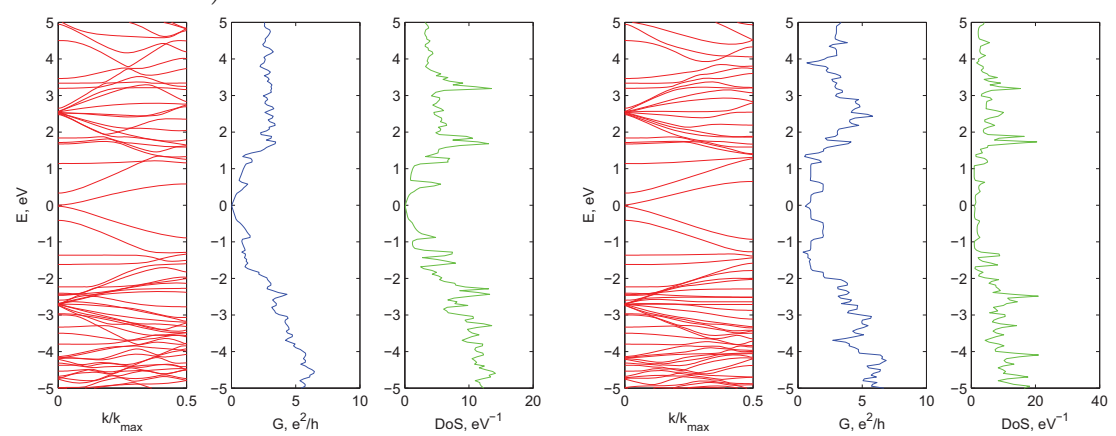

b) N-PFPA
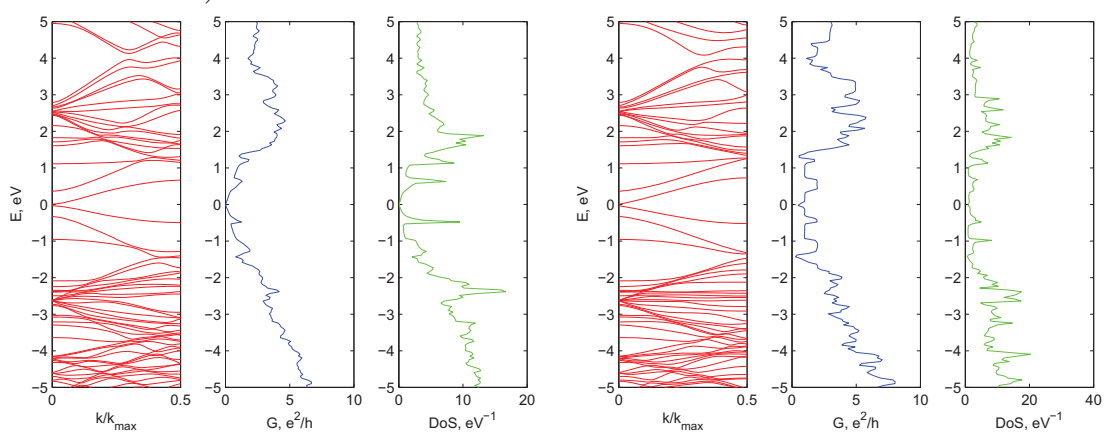

c) P-PFPA
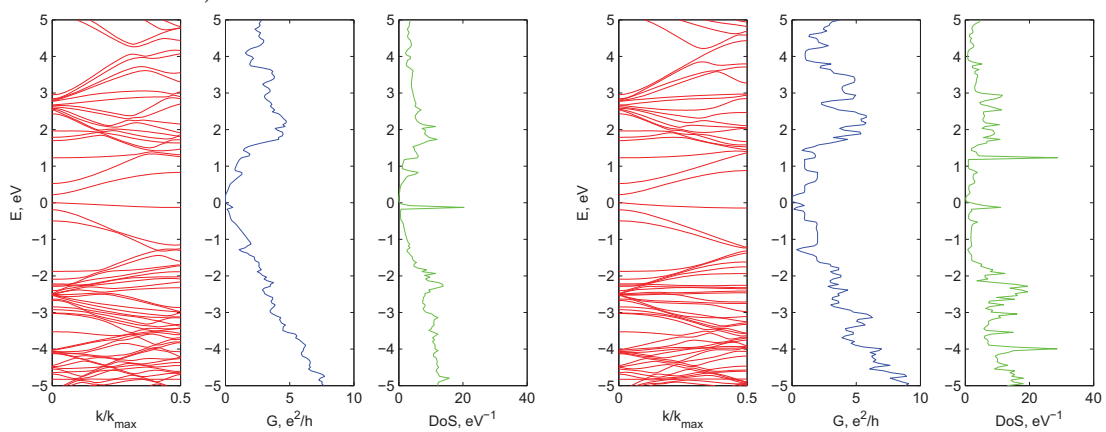

d) As-PFPA

Figure 6.18: Band structure, conductance, and density of states of B-, N-, P-, AsPFPA-FG in the $\Gamma-\mathrm{K}$ (top row) and $\Gamma-\mathrm{M}$ (bottom row) directions. 


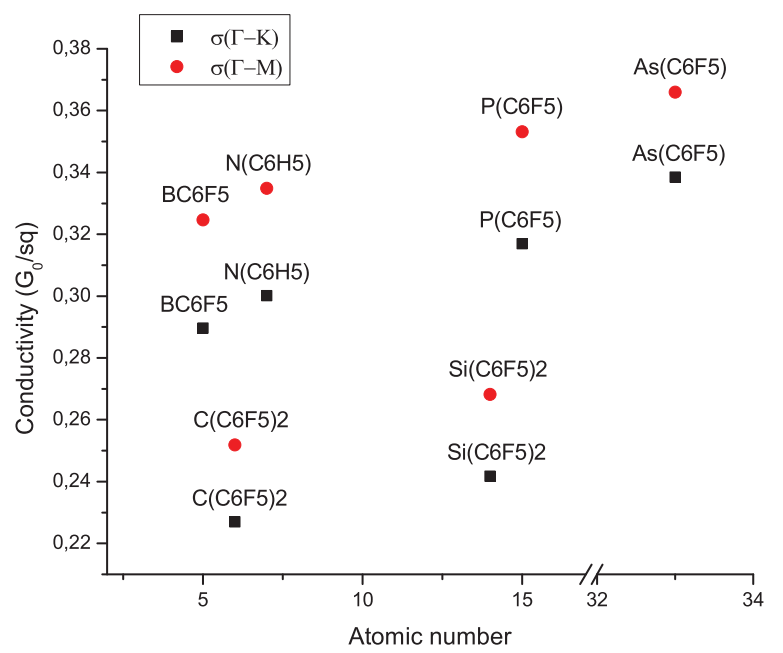

Figure 6.19: Conductivity of PFPA-FG as a function of the heteroatom.

trend observed for B, N, P, As. Functionalization with two PFPA groups, as it occurs in C- and Si-PFPA-FG, actually impedes electron transport even more than a single PFPA functionalization.

A general trend observed in figures 6.18 and 6.20 is that conductivity in the $\Gamma$-M direction is more ballistic (i.e. it deviates less from the eigenmodes count) than in the $\Gamma$-K direction. This repeats the picture readily observable in pristine graphene (Figs. 5.11 and 5.12), that it is not significantly disturbed despite functionalization. The BCD pictures for PFPA-FG show that PFPA functionalization as well as epoxyand amino- functionalization of graphene do not cause any doping, i.e. shift of the minimal conductance point to positive or negative values of electric bias. 

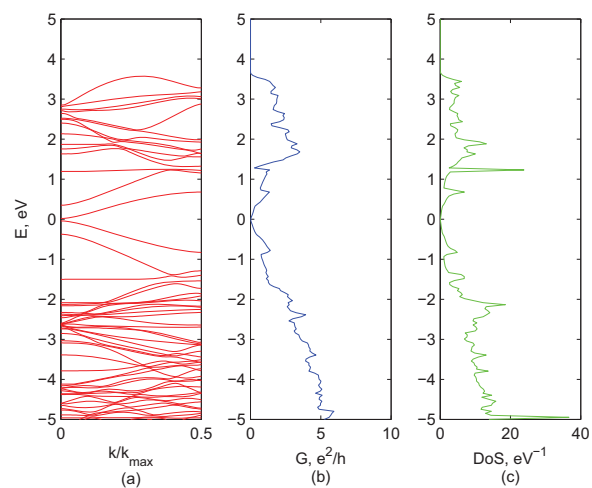

(a) C-PFPA-FG $\Gamma-\mathrm{K}$
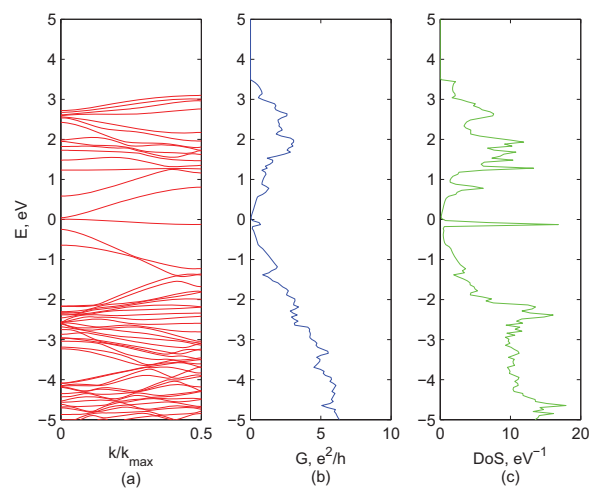

(c) Si-PFPA-FG $\Gamma-\mathrm{K}$
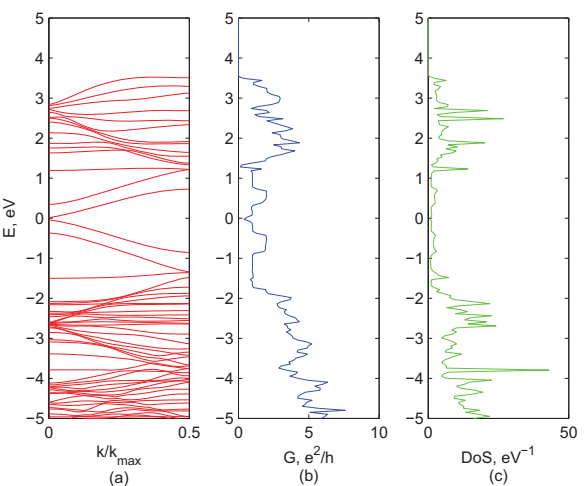

(b) C-PFPA-FG $\Gamma-\mathrm{M}$
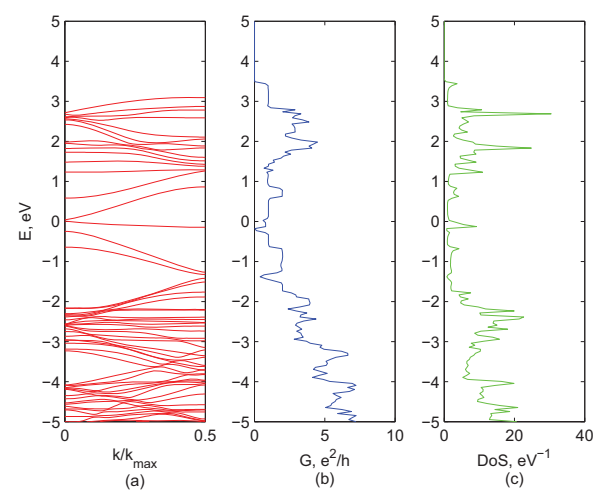

(d) Si-PFPA-FG $\Gamma-\mathrm{M}$

Figure 6.20: Band structure (a), conduction (b), and density of states (c) of C-, SiPFPA-FG in the $\Gamma-\mathrm{K}$ (left) and $\Gamma-\mathrm{M}$ (right) directions. 


\subsubsection{Comparison with experimental results}

Typical conductivity behavior in the vicinity of the former Dirac point demonstrate the same feature (Fig. 6.21(a)) observed in the experimental conductance measurements (Fig. 6.21(b)). Absolute values of conductance in Fig. 6.21(b) are irrelevant since they depend on the size of the sample, however the trend demonstrates that NPFPA functionalization, even though it locally disturbs $\pi$-system of pristine graphene still preserves linear conductivity behavior in the vicinity of the charge neutrality point.

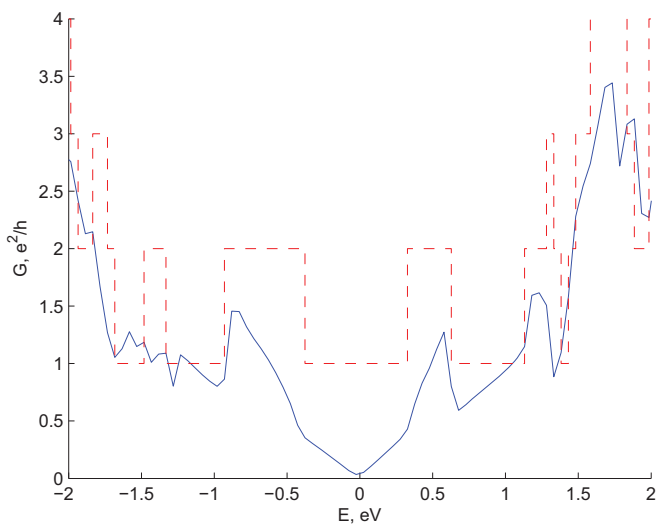

(a) $\Gamma-\mathrm{K}$

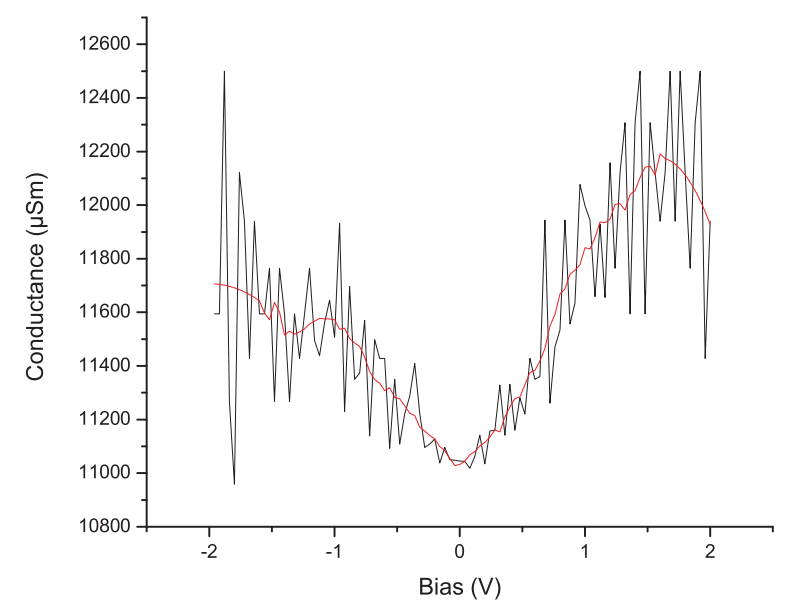

(b) $\Gamma-\mathrm{M}$

Figure 6.21: Comparison between theoretical (a) and experimental (b) conductance vs bias voltage curves.

This suggests that the disturbance of the conjugated electron system by PFPA functionalization is not very dramatic, indeed the average conductivity in the range of $\pm 5 \mathrm{~V}$ is just twice less for N-PFPA-FG vs pristine graphene. 


\subsection{Summary of covalent functionalization of graphene.}

In table 6.2 we summarize our numerical results: band gaps, average conductivities, charge transfer, obtained for epoxy-FG, amino-FG, and PFPA-FG. Among all covalent functionalizers considered in this chapter, the highest band gap is achieved in case of functionalization of graphene by PFPA molecules. Minimal conductivity

degradation remains about 50\%, and the highest band gap opened in N-PFPA-FG is $0.24 \mathrm{eV}$. This is about $100 \mathrm{kT}$ at room temperature and is already somewhat feasible for microelectronic applications. Since there are well established ways of production of PFPA-FG $[123,124,125]$ and it also has been demonstrated [134] that silene-PFPA (Fig. 6.8(b) and Fig. 6.9) can be used to immobilize graphene on Si-wafers, PFPA is probably the only industry-ready way to obtain functionalized graphene. 
Table 6.2: Summary of electronic properties produced by covalently functionalization of graphene.

\begin{tabular}{|c|c|c|c|c|}
\hline Structures & $\begin{array}{r}\text { Band gap } \\
(\mathrm{eV})\end{array}$ & $\begin{array}{r}\left\langle\sigma_{\Gamma-M}\right\rangle \\
\left(\frac{e^{2}}{h} / \square\right)\end{array}$ & $\begin{array}{l}\left\langle\sigma_{\Gamma-K}\right\rangle \\
\left(\frac{e^{2}}{h} / \square\right)\end{array}$ & $\begin{array}{r}\Delta Q \\
(\mathrm{e})\end{array}$ \\
\hline \multicolumn{5}{|l|}{ graphene } \\
\hline Hex & 0 & 0.7037 & 0.7037 & 0 \\
\hline Orth & 0 & 0.775279 & 0.775279 & 0 \\
\hline \multicolumn{5}{|l|}{ Epoxy } \\
\hline $\mathrm{C}$ & 27 & 0.237537 & 0.278921 & -0.025 \\
\hline $\mathrm{O}$ & 39 & 0.278077 & 0.300646 & -0.247 \\
\hline S & 41 & 0.28996 & 0.315328 & -0.411 \\
\hline \multicolumn{5}{|l|}{ Amino } \\
\hline $\mathrm{BH}$ & 14.55 & & & 0.339 \\
\hline $\mathrm{CH}_{2}$ & 36.15 & & & -0.977 \\
\hline $\mathrm{NH}$ & 2.47 & & & -0.321 \\
\hline $\mathrm{SiH}_{2}$ & 36.49 & & & 0.677 \\
\hline $\mathrm{PH}$ & 34.56 & & & 0.251 \\
\hline \multicolumn{5}{|l|}{ PFPA } \\
\hline $\mathrm{BC}_{6} \mathrm{~F}_{5}$ & 0 & 0.289671 & 0.324694 & 0.406 \\
\hline $\mathrm{C}\left(\mathrm{C}_{6} \mathrm{~F}_{5}\right)_{2}$ & 0.05442 & 0.226993 & 0.2519 & -0.054 \\
\hline $\mathrm{N}\left(\mathrm{C}_{6} \mathrm{H}_{5}\right)$ & 0.24489 & 0.300124 & 0.334886 & -0.314 \\
\hline $\mathrm{Si}\left(\mathrm{C}_{6} \mathrm{~F}_{5}\right)_{2}$ & 0.02721 & 0.241798 & 0.268179 & -0.032 \\
\hline $\mathrm{P}\left(\mathrm{C}_{6} \mathrm{~F}_{5}\right)$ & 0.05442 & 0.31693 & 0.353172 & 0.398 \\
\hline $\operatorname{As}\left(\mathrm{C}_{6} \mathrm{~F}_{5}\right)$ & 0.21768 & 0.338508 & 0.365968 & 0.087 \\
\hline
\end{tabular}

If band gap value is more than 10, it is in meV 


\section{CHAPTER VII}

\section{Haptic functionalization of graphene}


In this section I will discuss the electronic properties of graphene functionalized by haptic bonding to a metal atom. Haptic bonding significantly differs from the covalent one and thus is a subject of special interest for it does not cause rehybridization of carbon atoms and deplanarization of graphene sheet.

\subsection{Hapticity}

In the interaction of d-orbitals of a transition metal with ligands the following bonding processes can take place: $\sigma$-bonding, $\pi$-bonding, $\delta$-bonding, and $\pi$ backbonding. From those four types of bonds the first three represent conventional concepts inherited from organic chemistry, whereas the latter one ( $\pi$-backbonding or $\pi$-backdonation) is specific for binding of transition metals with multi-atomic $\pi$ conjugated ligands such as ethylene, carbonyl, benzene, etc. Backbonding is defined in the IUPAC Golden Book [138] as:

Backbonding: A description of the bonding of $\pi$-conjugated ligands to a transition metal which involves a synergic process with donation of electrons from the filled $\pi$-orbital or lone electron pair orbital of the ligand into an empty orbital of the metal (donor-acceptor bond), together with release (back donation) of electrons from a $d$ orbital of the metal (which is of $\pi$-symmetry with respect to the metal-ligand axis) into the empty $\pi^{*}$-antibonding orbital of the ligand.

This process can be schematically illustrated as:

This synergistic process of $\sigma-$ or $\pi$-donation of electron density from the ligand to the $d$-metal and release of excess electron density though $\pi$-backbonding is called haptic bonding. 
$\sigma$ bond:

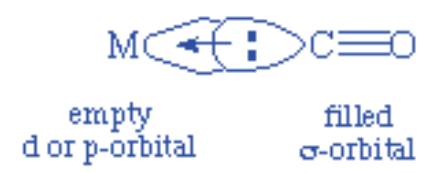

$\pi$ backbond:
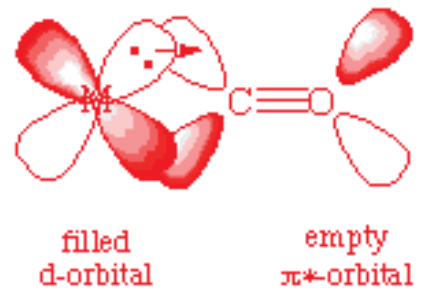

Figure 7.1: Interaction of a $d$-metal with a carbonyl molecule. Adopted from [139]

Among the first compounds discovered that demonstrate haptic bonding was ferrocene $\mathrm{Fe}\left(\mathrm{C}_{5} \mathrm{H}_{5}\right)_{2}$. Its structure consists of an iron atom "sandwiched" between two cyclopentene $(\mathrm{Cp})$ rings. This compound, despite absence of a traditional covalent bonding was found to be unusually stable. Followed by the discovery of ferrocene and studies of its rich chemistry, it was found that $\mathrm{Cp}$ is not the only ligand that can bind hapiticly to transition metals. Arene (Ar) rings, like $\mathrm{C}_{6} \mathrm{H}_{6}$ (possibly with substituents), were found to be a good haptic ligand due to alternating double and single $\pi$-bonds between carbon atoms in the ring structure. This alternation creates a conjugated resonant $\pi$-structure, that is very favorable for haptic bonding. Bis-arene metal sandwich complexes are known for all the transition metals, and their structure and chemistry resembles that of metallocenes. However, the even ligands are less strongly bonded to metals than the odd Cp ligand, and their chemistry suffers from easy decomplexation and is less rich than that of metallocenes [140].

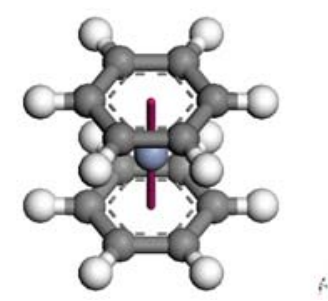

Figure 7.2: Structure of a Chromium(0)-bis-arene $\left(\mathrm{Cr}\left[\eta^{6}-\left(\mathrm{C}_{6} \mathrm{H}_{6}\right)_{2}\right]\right)$ molecule 
Our interest to Ar compounds is dictated by two following reasons:

- Metallocenes and metal-bis-arene compounds are known to be good electronic donors [141] and graphene shows the strongest interaction with electron-donor and acceptor molecules via molecular charge transfer [142],

- Geometrical structure of $\eta^{6}$ ( $\eta$ indicates how many ligand atoms participate in synergistic haptic bonding) compounds is similar to the honeycomb structure of graphene. More over, Elschebroich et.al. and Kündig et.al. [143, 144] demonstrated a possibility to synthesize Ar-compounds with condensed polyaromatics, and the metal atoms binding to the rings located at the periphery. (See Fig. $7.3)$

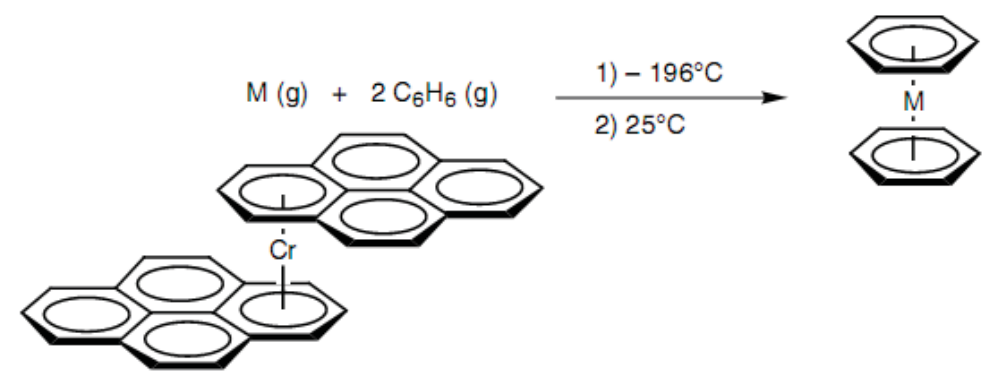

Figure 7.3: Structure of $\operatorname{Bis}\left(\eta^{6}\right.$-naphthalene)chrome(0) synthesized in [143]

\subsubsection{Electronic structure of metal-bis-arene molecules}

The study of metal arene compounds will begin with the molecular orbital (MO) of bis-arene-chrome $(0)\left(\mathrm{Cr}\left(\mathrm{C}_{6} \mathrm{H}_{6}\right)_{2}\right)$. The MO diagram below shows various metalring interactions: $\sigma(\mathrm{Ar} \longrightarrow \mathrm{Cr}), \pi(\mathrm{Ar} \longrightarrow \mathrm{Cr})$ and $\delta(\mathrm{Cr} \longrightarrow \mathrm{Ar})$. The $\sigma$ bond is constructed of $s$ and $d_{z^{2}}$ orbitals of Cr interacting with $a_{1 g}$ orbital of benzene, and Cr$p_{z}$ interacting with $a_{2 u}$. The HOMO of the complex results in a mixture of $d_{z^{2}}+a_{1 g} \rightarrow$ $a_{1 p}^{\prime}$ orbitals. The HOMO-1 is constructed from mixture of antibonding orbitals of 
benzene and $d_{x y}$ and $d_{x^{2}-y^{2}}$ orbitals of metal by the equation: $d_{x y}\left(d_{x^{2}-y^{2}}\right)+e_{2 g} \rightarrow e_{2 g}$. The LUMO is constructed from $d_{x z}$ and $d_{y z}$ orbitals by equation: $d_{x z}\left(d_{y z}\right)+e_{1 g} \rightarrow e_{1 g}^{*}$. From this analysis we conclude that the HOMO/LUMO system primarily consists of d-orbitals of the central atom. Thus we expect the same picture to be preserved in the band structure of periodic Ar-functionalized graphene.

The same diagram can be used to analyze stability of complexes with different metal atoms in the middle. In order to do so we need to consider the 18-electrons rule.

18 electrons rule: The 18-electrons rule reads as following: (see e.g. Minkin, Simkin, and Menyaev [145])

A stable complex (with electron configuration of the next highest noble gas) is obtained when the sum of the metal d-electrons, electron donated from the ligand, and the overall charge of the complex equals 18 .

18 electrons are required to fill 5 d-orbitals, 1 s-orbital and 3 p-orbitals of a transition metal and hence reach the configuration of a noble gas. As with most rules of thumb, the 18 electron rule is not always obeyed. So other metals can be sandwiched between Ar-rings. For instance, $\left[\mathrm{V}\left(\mathrm{C}_{6} \mathrm{H}_{6}\right)_{2}\right]$ can be isolated in both its neutral and monoanionic forms, these forms are isoelectronic to the stable complexes $\left[\mathrm{Cr}\left(\mathrm{C}_{6} \mathrm{H}_{6}\right)_{2}\right]^{+}$and $\left[\mathrm{Cr}\left(\mathrm{C}_{6} \mathrm{H}_{6}\right)_{2}\right]$, respectively. The complexes $\left[\mathrm{M}\left(\mathrm{C}_{6} \mathrm{Me}_{6}\right)_{2}\right](\mathrm{M}=\mathrm{Fe}, \mathrm{Co}, \mathrm{Ni})$ exist as neutral, mono- and dicationic complexes (although the structures of complexes that would have more than 20 valence electrons in the fully sandwich form do not exist as such, but partial decoordination reduces their valence electron count) [140]. When functionalizing a graphene sheet with the aforementioned complexes the 18-electrons rule becomes even weaker, since an infinite sheet can effectively distribute excessive 

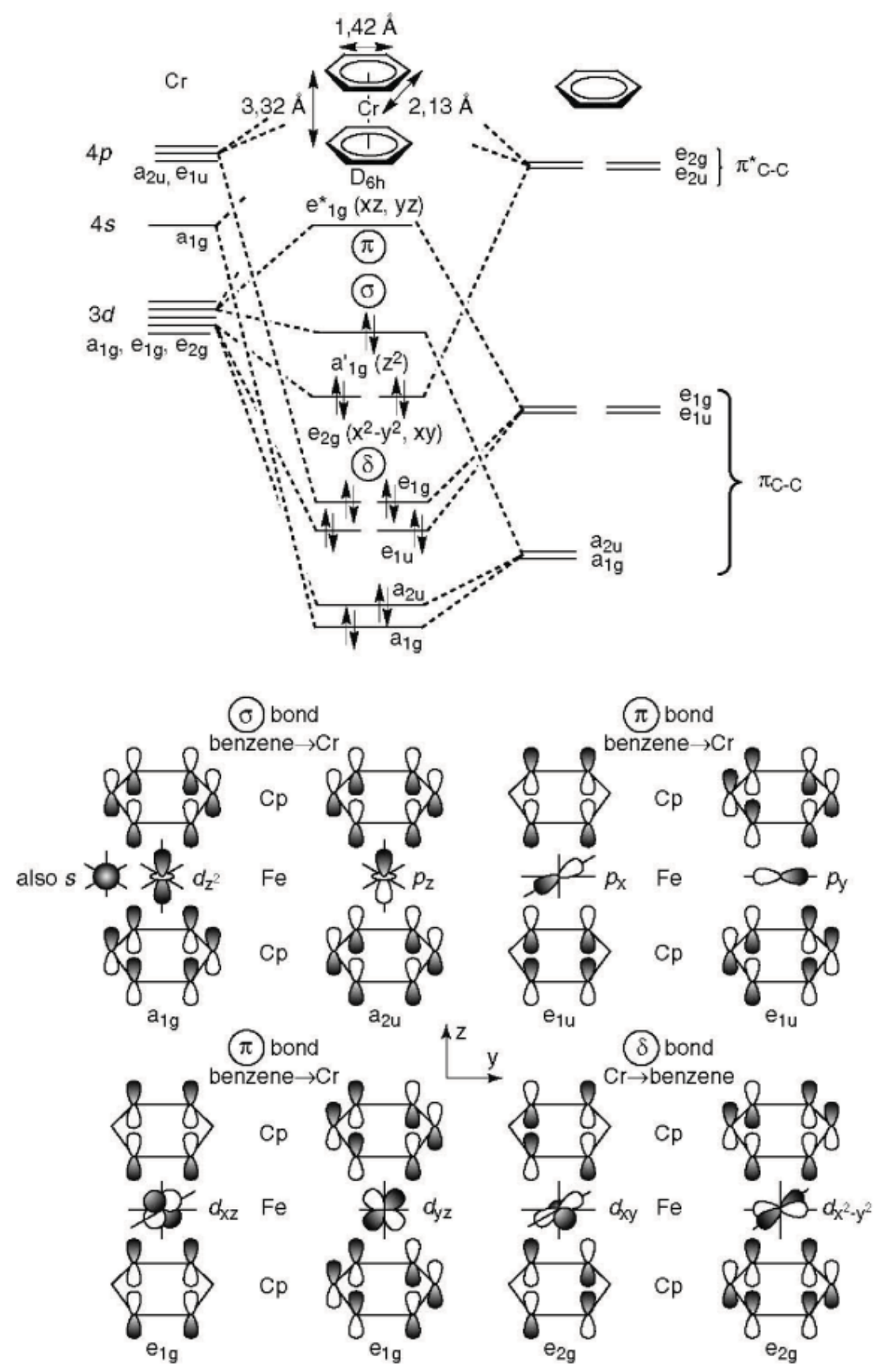

Figure 7.4: Molecular orbital diagram of $\mathrm{Cr}\left(\mathrm{C}_{6} \mathrm{H}_{6}\right)_{2}$ (top) and interactions of the benzene orbital with the $\mathrm{Cr}$ orbitals of appropriate symmetry to form $\mathrm{Cr}\left(\mathrm{C}_{6} \mathrm{H}_{6}\right)_{2}$ (bottom). Adapted from [140]

electron density or donate missing electrons to complete the orbitals. But nevertheless an extensive study of binding energy is necessary when deviating from the 
18-electrons rule to ensure stability of the structures under inspection.

By a careful selection of the center atom we can do fine tuning of mutual level location and, afterwards, transferring those properties to functionalized graphene. This tailoring of the band structure is especially important when designing devices for nanophotonic application, such as filters, bolometers, infrared LEDs, and phototransistors.

\subsection{Metal-arene functionalization}

Basing on the discussion above, we undertook a preliminary study of Cr-arene functionalized graphene. Chromium is chosen because $\mathrm{Cr}\left(\mathrm{C}_{6} \mathrm{H}_{6}\right)_{2}$ has electron count of exactly 18 electrons, and thus is expected to be more stable than other metal derivatives.

Atomic models possessing one functionalizing molecule per 2x2, 3x3, 4x4, 5x5, 6x6, and 8x8 graphene supercells (hex22 - hex88 respectively) were designed, after which their geometry was optimized with respect to the minimization of the total energy. Band structures of all aforementioned supercells of metal-arene fuctionalized graphene (MAFG) as well as pristine graphene were computed for the models with optimized geometry. Band structures were then aligned with respect to the position of the Fermi level, that allows straightforward estimation of the functionalization effects.

An important property of the MA-induced perturbation of the band structure is that alteration in the electronic structure of graphene increases with increasing MA functionalization concentration. We have investigated the functionalization of graphene at higher adduct concentration by including three more MA functional groups in the unit cell (see Fig. 7.7b). Due to the limitations imposed by the nature of the 
DFT calculations we cannot study the variation of the electronic properties imposed by continuously changing concentration of functionalizing molecules. This would require dealing with huge supercells. Two aforementioned geometries correspond to one functionalizing molecule per 6x6 and 3x3 graphene supercells. An important idea is to demonstrate the ability to tweak the band gap by varying the concentration, possibly even in the broader limits than discussed here. Exact positions of functionalizing molecules inside the unit cell are not important since there are many ways to redefine the lattice.

For the calculation of the band structure the k-path was chosen M- $-\mathrm{K}$ with 12 and $9 \mathrm{k}$-points on each segment correspondingly. Due to the supercell geometry (as it follows from Sec. 2.6) BZ folding occurs: for every supercell that is not $2^{n} \times 2^{n}$, the Dirac point $K$ of the subcell maps onto $\Gamma$ of the supercell, otherwise the Dirac point $K$ of the subcell maps onto the Dirac point of the supercell ${ }^{1}$. The $\Gamma$ point remains invariant with respect to folding.

The 2x2 band structure significantly deviates from other supercells, the reason for that is sterical confinement of benzene rings. The distance between the hydrogen atoms from neighboring rings in the initial configuration is less than $1 \AA$ (Fig. 7.6a), and thus some relaxation takes place: benzene rings bend and arrange themselves in a parquet pattern (Fig. 7.6b). This makes the band structure of the $2 \mathrm{x} 2$ supercell quite distinct from that of the other supercells.

As it can be seen from Fig. 7.5, 3x3 and 6x6 functionalization provide the widest band gap. Therefore, for further consideration, we will carefully examine the cases of $3 \times 3$ and $6 \times 6$ supercell. In order to be able to directly compare band structures in the aforementioned supercells, the 3x3 supercell can be equivalently represented

\footnotetext{
${ }^{1}$ even despite $K_{\text {sub }}$ and $K_{\text {super }}$ are located in virtually different positions of the reciprocal space, but the folding procedure makes them spatially degenerated
} 


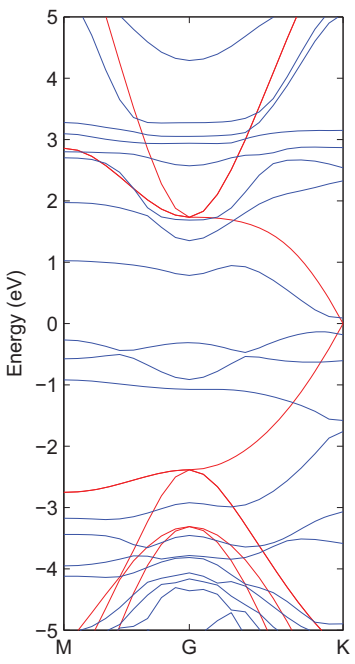

(a) $2 \times 2$ supercell

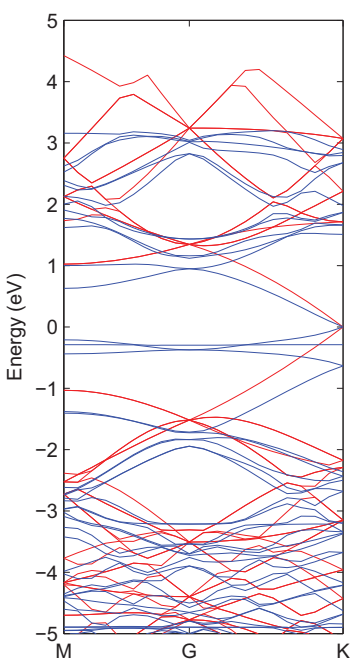

(d) $5 \times 5$ supercell

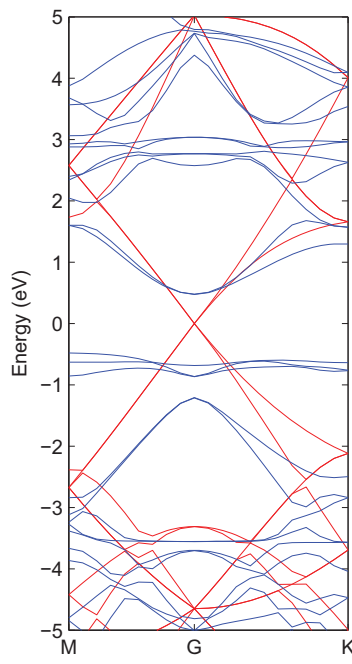

(b) 3x3 supercell

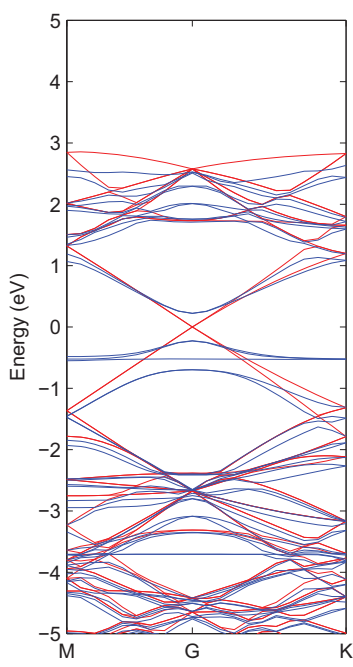

(e) 6x6 supercell

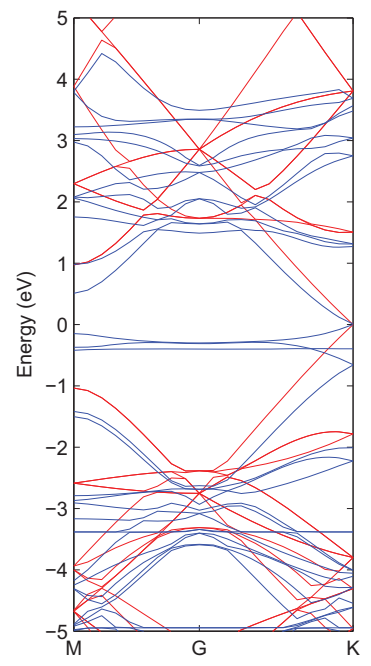

(c) $4 \times 4$ supercell

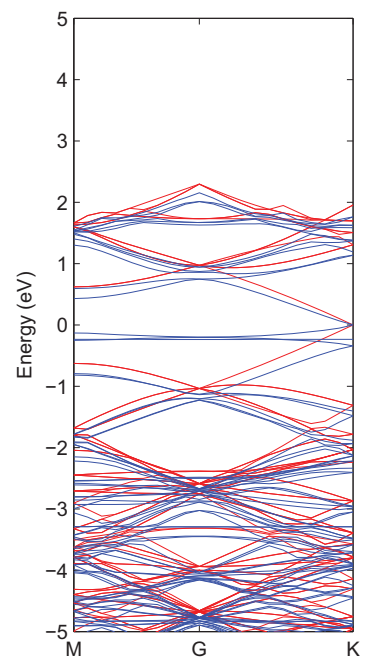

(f) $8 \times 8$ supercell

Figure 7.5: Band structures of pristine (red) and functionalized (blue) graphene, computed with different degree of functionalization: one MA molecule per $\mathrm{XxX}$ graphene supercell.

as a $6 x 6$ supercell with four functionalizing molecules per unit cell (Fig. 7.7). Only these two geometries are selected for further studies of the effect of the electron 


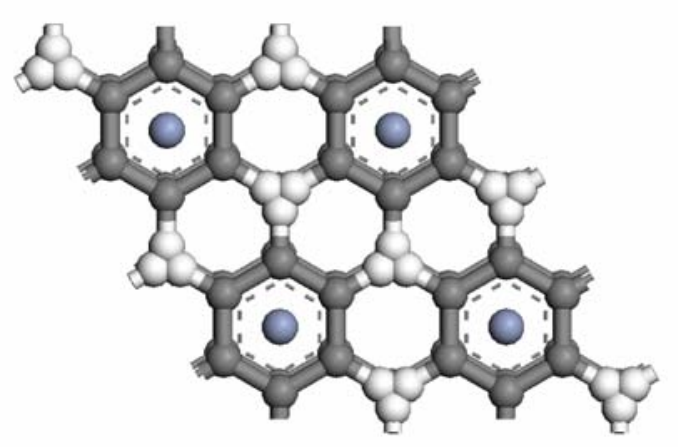

(a)

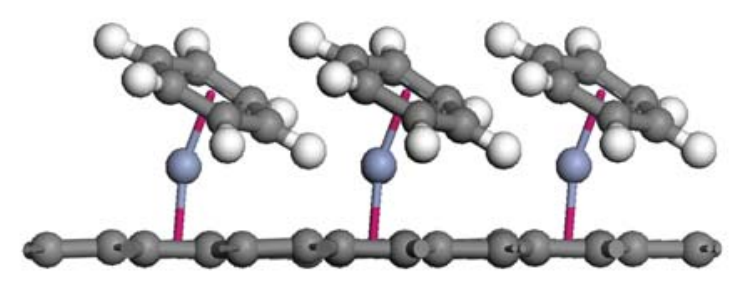

(b)

Figure 7.6: 2x2 MAFG (a) initial geometry, top view and (b) optimized geometry, side view

configuration of the central atom on the band structure. Further, the structure with one functionalizing molecule per $6 \mathrm{x} 6$ supercell is abbreviated as 1-MAFG, the same with four functionalizing molecules: 4-MAFG. However, everything that is said here about $3 \times 3$ and $6 \times 6$ supercells can be applied to other degrees of functionalization as well.

We also studied the effect of of the central metal atom for the following 3d-metals: Ti, V, Cr, Mn, Fe (Zn and $\mathrm{Cu}$ have closed 3d shell and therefore are ignored) in two aforementioned configurations: 1-MAFG and 4-MAFG.

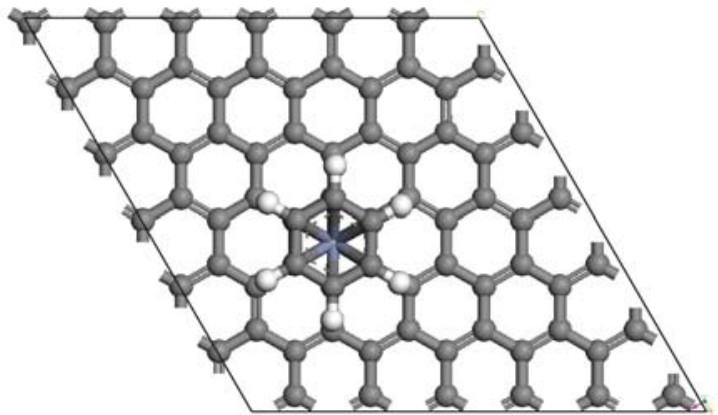

(a)

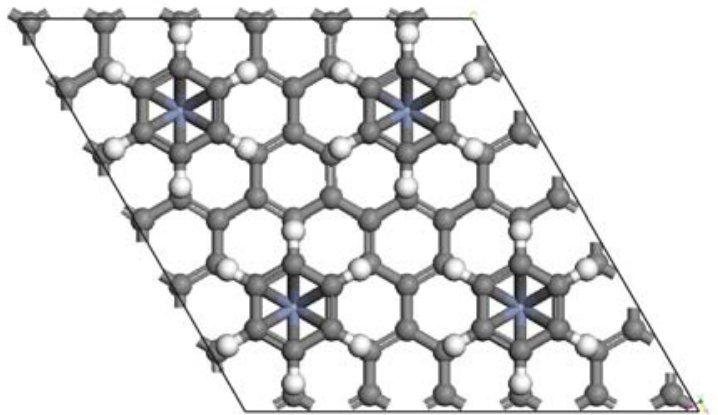

(b)

Figure 7.7: Ball-and-stick presentation of optimized structures of MA-functionalized graphene (top view) with one (a) and four (b) MA adducts per 6x6 graphene supercell. 
The structural parameters of all MAFG structures were optimized by means of the standard DFT procedure as described in appendix A.1.

Table 7.1: Geometric parameters of free MA molecules and MA molecules bound to graphene sheet.

\begin{tabular}{llllll} 
Metal atom & $\mathrm{Ti}$ & $\mathrm{V}$ & $\mathrm{Cr}$ & $\mathrm{Mn}$ & $\mathrm{Fe}$ \\
\hline$\angle(\mathrm{CMC}){ }^{\circ}(\mathrm{MA})$ & 103.477 & 101.397 & 99.76 & 101.285 & 104.353 \\
$\mathrm{M}-\mathrm{C}(\mathrm{a})(\AA)$ & 2.312 & 2.255 & 2.211 & 2.244 & 2.314 \\
\hline$\angle(\mathrm{CMC}){ }^{\circ}(1-\mathrm{MAFG})$ & 105.411 & 101.433 & 101.183 & 99.748 & 97.475 \\
$\mathrm{M}-\mathrm{C}(\mathrm{a}) / \mathrm{M}-\mathrm{C}(\mathrm{g})(\AA)$ & $2.361 / 2.368$ & $2.295 / 2.310$ & $2.235 / 2.266$ & $2.171 / 2.266$ & $2.186 / 2.358$ \\
\hline$\angle(\mathrm{CMC}){ }^{\circ}(4-\mathrm{MAFG})$ & 105.346 & 102.886 & 100.991 & 100.198 & 102.835 \\
$\mathrm{M}-\mathrm{C}(\mathrm{a}) / \mathrm{M}-\mathrm{C}(\mathrm{g})(\AA)$ & $2.335 / 2.391$ & $2.26 / 2.334$ & $2.216 / 2.274$ & $2.161 / 2.298$ & $2.198 / 2.394$
\end{tabular}

(a) stands for bonding to the topping arene molecule, (g) - for the underlying graphene sheet

As can be seen from Table 7.1, the bonding to an infinite graphene sheet slightly changes the structural features with respect to the free $\mathrm{M}\left[\eta^{6}-(\mathrm{Ar})_{2}\right]$ molecule. General trends in the bonding lengths demonstrate the following features: the M-C(graphene) bond lengths remain about 3\% longer than the one in the free molecule; M-C(arene) bonds, however, remain almost unchanged with respect to the free molecules for $\mathrm{Ti}-\mathrm{Cr}$ metals and become about 3\% shorter for Mn and Fe. (See Fig. 7.8)

The reason for the extreme behavior of the bond lengths can be explained by considering a molecular orbital representation of the MAs. The usage of molecular orbitals (instead of Wannier functions for a periodic system) is justified because we assume little interaction between the molecules from the neighboring cells. In the $\left.\operatorname{Cr}\left[\eta^{6} \text {-(arene }\right)_{2}\right]$ electrons fully occupy $a_{1 g}$ binding orbital, whereas adding additional electrons, as happens in $\mathrm{Mn}$ and Fe leads to partial population of the anti-bonding twice degenerate $e_{1 g}^{*}$ orbital. This orbital is composed of $4 \mathrm{p}$ and $4 \mathrm{~s}$ atomic orbitals of the metal and anti-bonding $\pi^{*}$ orbitals of graphene and arene (See Table 7.2 and Fig. 7.4), which manifests itself in the attempts of the molecule to lower its excessive 
energy by structural reconstruction.

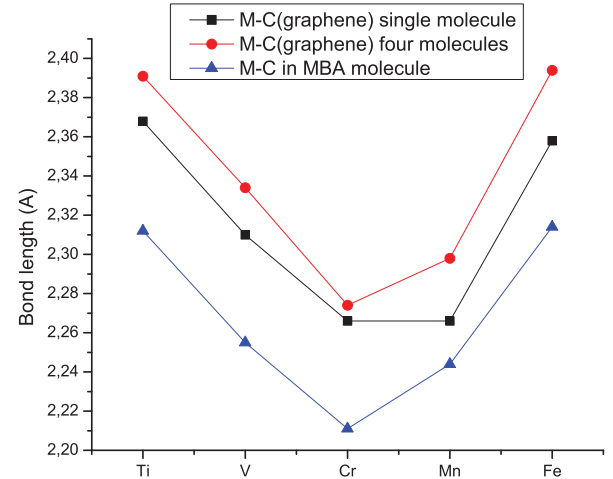

(a)

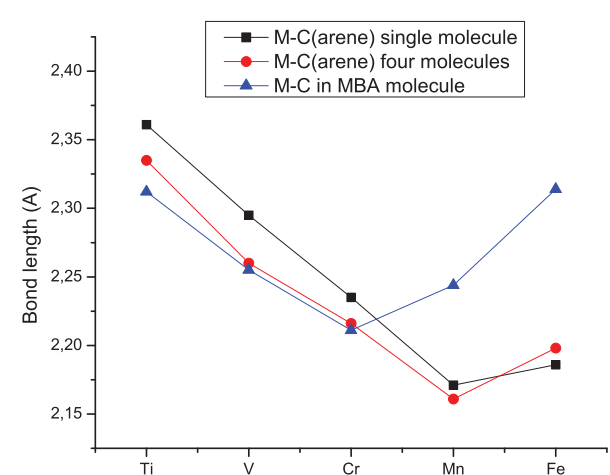

(b)

Figure 7.8: Geometrical parameters of the MA-FG (a) M-C(arene) bond length, (b) $\mathrm{M}-\mathrm{C}$ (graphene) bond length.

The same mechanism does not affect $\mathrm{C}$-M bonds since the conjugated $\pi$-system of the graphene sheet can effectively redistribute additional electron density donated by the metal atom through the $\pi$-backdonation mechanism, and thus decrease the number of electrons on the M-C(graphene) bond, compared to an isolated molecule where this delocalization cannot effectively take place, and thus, weaken it. Deviation from the single molecule behavior for the M-C(arene) bond (Fig. 7.4) is hardly observed for $\mathrm{Ti}, \mathrm{V}$, and $\mathrm{Cr}$. In the case of $\mathrm{Mn}$ and $\mathrm{Fe}$ compounds, as mentioned above, demonstrate about 3\% shortening of the bond. We relate this distortion to the Jahn-Teller effect: unpaired electrons in the Mn and Fe compounds occupy doubly degenerate $e_{1 g}^{*}$ level, and therefore Mn and Fe compounds undergo geometrical distortion that removes degeneracy. This asymmetry in bond length also leads to the difference in $\mathrm{C}($ arene)-M-C(graphene) angles: for Ti-Cr they exceed those for free molecule, but Mn-Fe are less. This phenomenon results in less mixing of the localized atomic d-orbitals and leads to creation of narrow bands in the band structure of 
MA-FG, decreasing the band gap compared to corresponding Ti-Cr compounds

\subsection{Electronic structure MAFG}

In this section we present the main result of haptic functionalization: a gap opening in the band structure of MAFG.

\subsubsection{Electronic configuration of 1-MAFG and 4-MAFG}

The graphene-metal interaction in haptic functionalization has direct consequences on electronic properties of graphene. As previously reported, functionalization of graphene with radical (primarily hydrogen, epoxide, and nitrene) groups locally disrupt the planarity of the graphene sheet, changing local hybridization form $s p^{2}$ to $s p^{3}$ geometry $[120,81,121]$ which induces an $s p^{3}$-type defect-like state near the Fermi level ${ }^{2}$. In our case, however, the graphene sheet is not distorted in the z-direction and thus re-hybridization of carbon atoms does not occur. The local bonding configuration is, however, significantly affected by the electronic structure of the functionalizing atom, especially its d-electrons, and its energy states that lie close to the Fermi level. This is similar to the situation with $s p^{3}$-type "impurity" states for the radical functionalization. Partially occupied highly localized d-orbials near the Fermi level cause repulsion of the $\delta$-bands, causing the energy band of pristine graphene to be shifted away from the Fermi level due to the $\pi$-d interaction.

\subsubsection{Band structure of 1-MAFG}

The calculated band structures for CrAr-functionalized graphene are compared with pristine graphene in Figure 5.5. It is readily observable that after haptic func-

\footnotetext{
${ }^{2}$ Fermi level is defined in intrinsic semiconductors in the middle of the band gap, however, by the majority of the DFT programs it is conventionally placed at the top of the valence band
} 
Table 7.2: Electronic configuration of the metal atoms in the MA, and the corresponding energy gap opening in the MA-FG as the result of functionalization.

\begin{tabular}{lccccc} 
Metal atom & $\mathrm{Ti}$ & $\mathrm{V}$ & $\mathrm{Cr}$ & $\mathrm{Mn}$ & $\mathrm{Fe}$ \\
\hline Electron count & 16 & 17 & 18 & 19 & 20 \\
Electronic configuration: & & & & & \\
$e_{1 g}^{*}(y z, x z)$ & - & - & - & $\uparrow-$ & $\uparrow \uparrow($ or $\downarrow \downarrow)$ \\
$a_{1 g}^{\prime}\left(z^{2}\right)$ & & $\uparrow$ & $\uparrow \downarrow$ & $\uparrow \downarrow$ & $\uparrow \downarrow$ \\
$e_{2 g}\left(x^{2}-y^{2}, x y\right)$ & $\uparrow \downarrow \uparrow \downarrow$ & $\uparrow \downarrow \uparrow \downarrow$ & $\uparrow \downarrow \uparrow \downarrow$ & $\uparrow \downarrow \uparrow \downarrow$ & $\uparrow \downarrow \uparrow \downarrow$ \\
\hline Number of unpaired electrons & 0 & 1 & 0 & 1 & 2 \\
\hline$E_{g}(1-\mathrm{MAFG})(\mathrm{eV})$ & 0.40815 & 0.10884 & 0.38094 & 0.29931 & 0 \\
$E_{g}(4-\mathrm{MAFG})(\mathrm{eV})$ & 0.32652 & 0.78909 & 0.8163 & 0 & 0.48978
\end{tabular}

tionalization the linear dispersion law of pristine graphene at the Dirac point is entirely broken. The $\pi$ and $\pi^{*}$-bands preserve their arrangement in the functionalized graphene, however the distance between them grows from about $1 \mathrm{eV}$ in $1-\mathrm{MAFG}$ to $1.25 \mathrm{eV}$ in $4-\mathrm{MAFG}$. This growth is attributed to the shift of the $\pi^{*}$ band by $+0.25 \mathrm{eV}$ relative to the 1-MAFG) The analysis of the density of states calculated for different atoms and projected on different angular momenta demonstrates that a system of pure (with no $\pi$-admixture) localized d-bands of the metal is now located between the $\pi$ - and $\pi^{*}$-bands of graphene, preventing them from crossing. These d-bands cause strong repulsion and are now responsible for the opening of the gap between the bands produced by the metal atomic levels. These electronic properties of MAfunctionalized products contrast from the $s p^{3}$ re-hybridization and loss of $\pi$-electrons found upon addition of acceptor chemical groups or metals in other functionalization schemes $[146,147,131]$. The fact that the band gap strongly depends on the nature of the functionalizing metal atom confirms our idea about the importance of the number of d-electrons or the modification of the band structure. Both occupied and empty d-levels of the metal form flat bands close to the top of the valence band. These "impurity" states are probably responsible for bringing the strongest contribution to the band repulsion, more than the d-admixture to the former pure $\pi$-and $\pi^{*}$-bands. A higher number of $\mathrm{d}$-bands complicates the picture. The distance between the $\pi$-bands 
in 1 -VAFG at the $\Gamma$-point is about $0.8 \mathrm{eV}$ but "impurity" levels decrease it ten times to $0.08 \mathrm{eV}$. The distance between the d-levels in $1-\mathrm{MnAFG}$ is about $2.7 \mathrm{eV}$ which is much more than the distance between $\pi$-bands, thus the highest occupied molecular orbital (HOMO) and the lowest unoccupied molecular level (LUMO) of 1-MnAFG line up with the $\pi$ - and $\pi^{*}$-bands of graphene. As the number of electrons in the system increases, the Fermi level drifts up causing a transition from a semiconductor to a metallic state. The band alignment of 1-MnAFG and 1-FeAFG are almost the same, however, due to the extra electron of Fe the Fermi level is becomes coincident with the former $\pi^{*}$-band making the iron compound semimetallic. Additional flat d-bands produced by the localized electrons of the metal atoms in the MAFG can be successfully utilized to mimic dopant levels of conventional semiconductors.

\subsubsection{Band structure of 4-MAFG}

As the concentration of functionalizing molecules increases (i.e. by transition from 1-MAFG to 4-MAFG) repulsion between the $\pi$-bands (graphene) increases as well, leading to the wider band gap opening. The extracted energy gap is $0.44 \mathrm{eV}$ and $0.98 \mathrm{eV}$ for one and four $\mathrm{Cr}(\mathrm{Ar})$ adducts (i.e. CrAFG and 4-CrAFG), respectively, on a graphene unit cell consisting of 72 graphene-carbon atoms. Same trend is observed in transition form 4-MnAFG to 4-FeAFG. The later becomes metallic for the very same reason: since the Fermi level is located higher in the iron compound, it becomes metallic even despite very close similarity of the band alignment between 4-MnAFG and 4-FeAFG. Band diagrams of all substances under consideration are presented in Fig. 7.9. Again, as before, BZ folding in a $6 \times 6$ supercell leaves directions $\Gamma-\mathrm{K}$ and $\Gamma-\mathrm{M}$ invariant. Thus band structure calculated along these directions actually reflects the situation in an unfolded BZ.

Closer analysis of band alignment demonstrates that gap opening can be primar- 

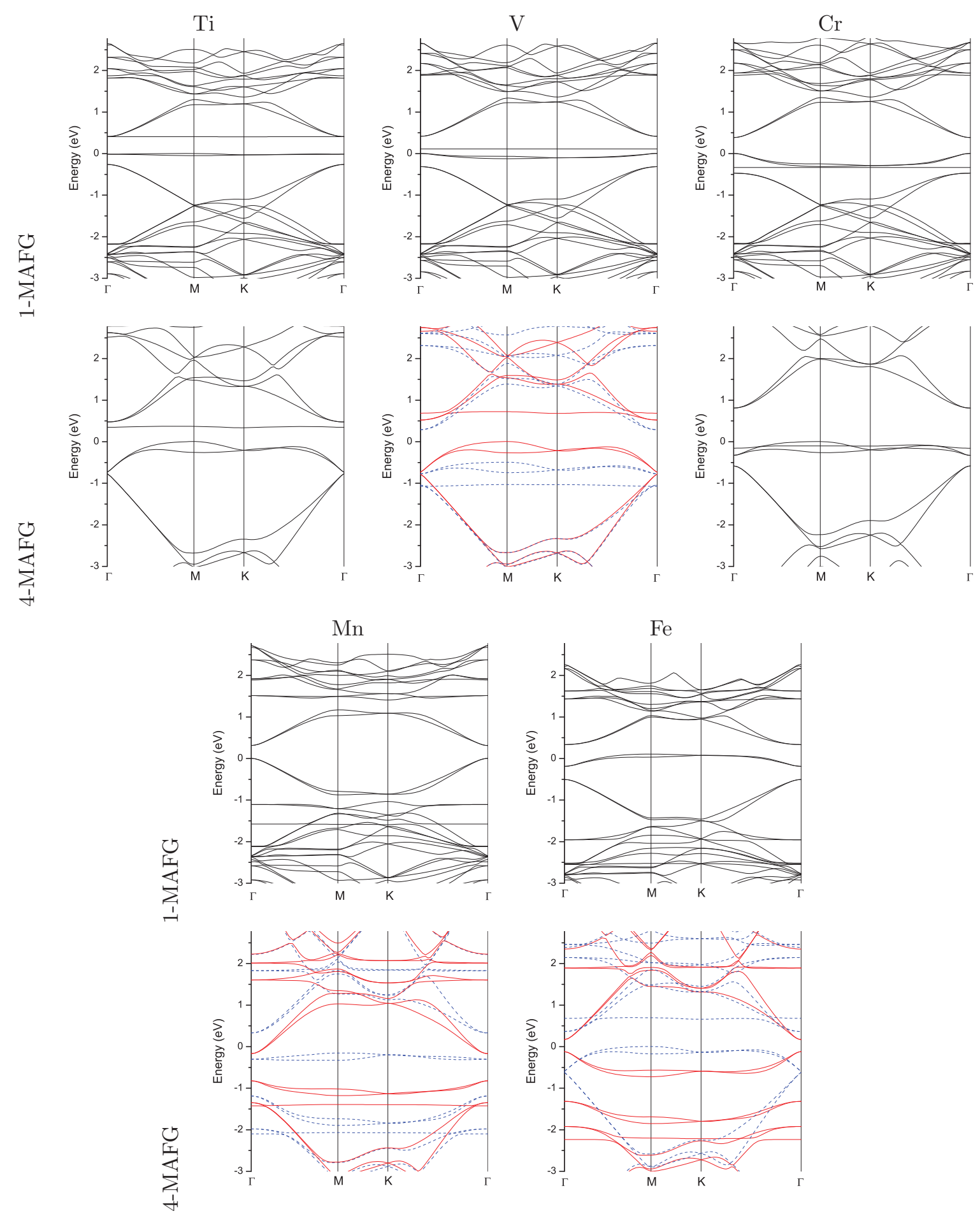

Figure 7.9: Band structures of 1-MAFG and 4-MAFG, where M=Ti, V, Mn, Fe. Red and blue coloring correspond to spin up and spin down bands. Energy reference level coincides with the position of Fermi level 
ily attributed to the interaction of the d-electrons with the $\pi$-conjugated system. Although the carbon atoms in graphene connecting to MA essentially retain a flat band configuration corresponding to $s p^{2}$ hybridization, additional $\pi$-d interaction is nevertheless present. Local modification of the original $\pi$-conjugation in the vicinity of the metal atom is manifested by re-hybridization, i.e. the highest occupied molecular orbital (HOMO) and the lowest unoccupied molecular orbital (LUMO) of MAFG now are formed by the $\pi$-backbonding mechanism. In some cases, however, this mechanism appears to be very weak, leaving d-orbitals of the metal atom almost unhybridized. This rehybridization, however, contrasts to conventional $s p^{2} \rightarrow s p^{3}$ rehybridization because it occurs without major geometrical distortion of underlying graphene sheet. Accordingly, carrier scattering can be substantially regarded as due to electrostatic interaction similar to that observed for ionized dopant impurities in conventional semiconductors rather than due to localized defect states. As a consequence, it is to be expected that mobility degradation will be much less in MAFG than in covalently functionalized graphene for which significant non-planarity of the graphene sheet is unavoidable. Indeed, this is a crucial difference and was the original motivation for considering this type of functionalization, since it seems rather obvious that preservation of aromaticity vs $s p^{2}$ hybridization, should result in less degradation of carrier transport properties. In order to confirm this hypothesis, we undertook studies of the NEFG electron transport in MAFG structures.

\subsection{Transport in MAFG structures}

\subsubsection{Molecular orbitals of MAFG structures}

The probability densities of the corresponding HOMO/LUMO at the band center ( $\Gamma$ point) are shown in Fig. 7.10. Different atoms demonstrate different mixing of 
atomic orbitals that take part in the formation of HOMO and LUMO. The HOMO of 1-CrAFG and 1-MnAFG (not shown) were constructed by the $\sigma$-type donation mechanism (empty d-orbital is interacting with filled $\pi$-orbital). The LUMO of these compounds and 1-VAFG, in turn, demonstrate $\pi$-type back-donation behavior (filled d-orbital interacts with an empty $\pi^{*}$-orbital). The HOMO of 1-VAFG is entirely represented by the $d_{z^{2}}$ orbital of the metal atom. The MO picture of 1-FeAFG is somewhat different from the other atoms: $\pi$-type back-donation for HOMO and $\sigma$-type donation for LUMO. This is to be contrasted with 4-MA functionalization. As can be seen in Fig. 7.10, an increase of adduct concentration impedes the donation mechanism causing the HOMOs of 4-CrAFG, to consist of the unhybridized $d_{z^{2}}$ orbitals of the metal atom and the LUMO of the $\pi$-type back-donation MOs. Molecular orbitals of other MAFG structures are not shown here due to space limitations. However, the donation mechanism changes depending on the number and energy of filled d-orbitals of the central atom. Proportional increase of functionalizing molecules causes a change of the amount of $\pi$-conjugated bonds broken due to the back-bonding mechanism. This correlates with the associated increase of the band gap and thus provides support of the suggested scenario of the d-level-induced band gap opening.

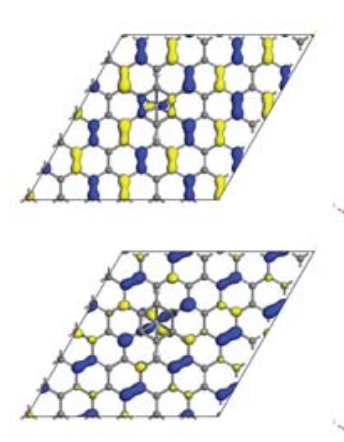

(a)

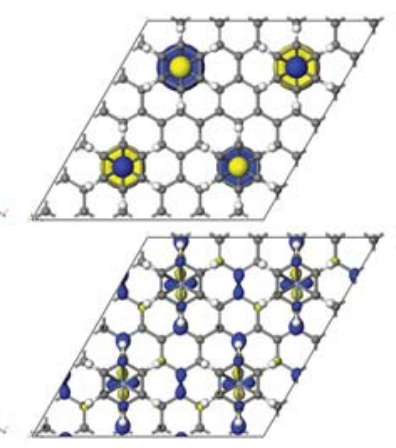

(b)

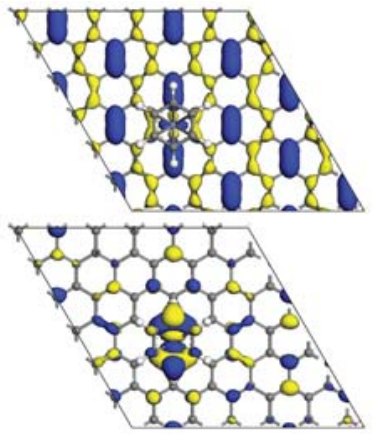

(c)

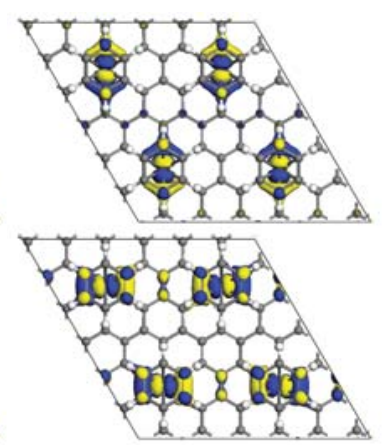

(d)

Figure 7.10: Molecular orbitals (HOMO- top row, LUMO bottom row) for 1-CrAFG (a), 4-CrAFG (b), 1-FeAFG (c), 4-FeAFG (d) 


\subsubsection{Wannier functions of MAFG structures.}

Trial Wannier functions were initialized as two subsets: graphene subset is initialized as a set of $\sigma$-type orbitals located between carbon atoms and the dangling $p_{z}$ orbitals. However, unlike WFs associated with covalent bonds in epoxy, amino, and PFPA structures, The functionalizing set of WFs consisted of a full valence AOs of the central metal atom, i.e. $5 d+3 p+1 s$ orbitals. WF of the terminating benzene ring were initialized in the same way as graphene: $5 \sigma$-type bonds and $6 p_{z}$-orbitals for carbon atoms. Hydrogen atoms were AOs not included in the WF manifolds since they are already counted by $\mathrm{C}-\mathrm{H} \sigma$-subsets. After the localization procedure the WFs spreads were quite uniformly suggesting that the set chosen is correct since it adequately describes decomposition of delocalized Bloch orbitals into localized Wannier orbitals An example of the WF expansion in 1-CrAGF structure is given in Fig. 7.11.

The structure of WFs demonstrates a picture very similar to the one delivered by the MO picture ${ }^{3}$. Mixing between the states occurs in accordance with the correlation diagram (Fig. 7.4), by means of the backdonation mechanism, yielding efficient mixing between $p_{z}$ orbitals of graphene and d-orbitals of functionalizing metal.

The volume enclosed by isosurfaces, plotted at different levels of probability is significantly smaller for p-orbitals in comparison with the d-orbitals, reflecting a wellknown fact that d-electrons are stronger localized than p-electrons.

Wannier functions of carbon atoms are distorted in the vicinity of the functionalizing metal atom, that reflects their response the the need to donate extra electron density, associated with the delocalized $\pi$-system of graphene, which it transferred to the metal atom as the result of functionalization.

\footnotetext{
${ }^{3}$ As it is mentioned in Sec. 5.1.2, MOs is a special case of WFs if only one k-point $(\Gamma)$ is considered. In case of relatively large supercells, their BZs are fairly small and therefore, one k-point often provides sufficiently good description of the electronic structure.
} 


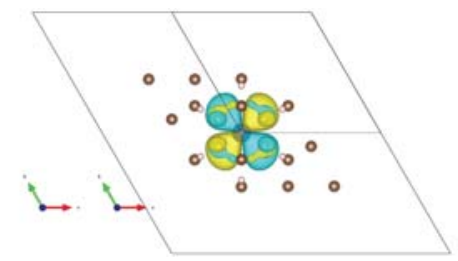

(a) $d_{x y}$

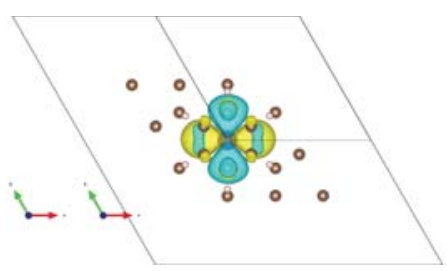

(d) $d_{x^{2}-y^{2}}$

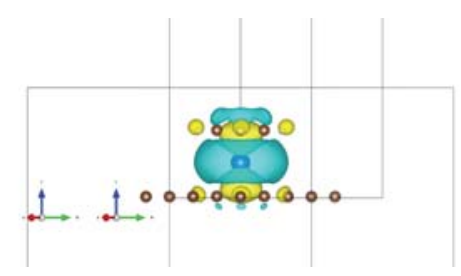

(g) $d_{z}$

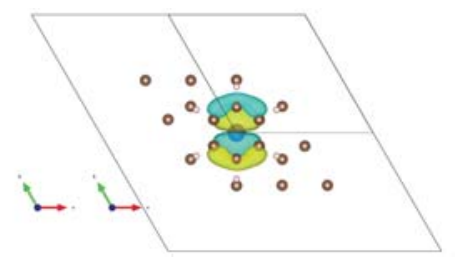

(b) $d_{x z}$

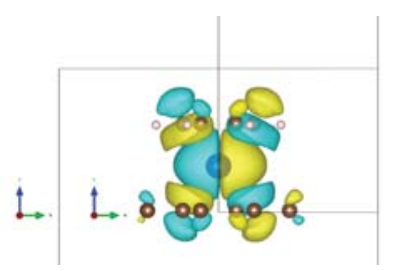

(e) $p_{x}$

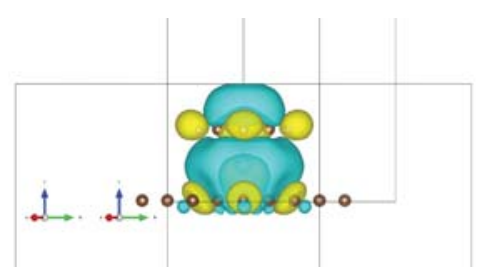

(h) $p_{z}$

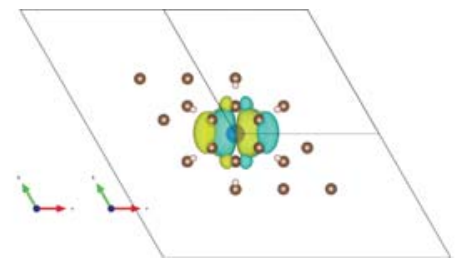

(c) $d_{y z}$

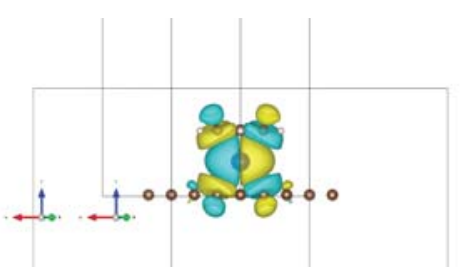

(f) $p_{y}$

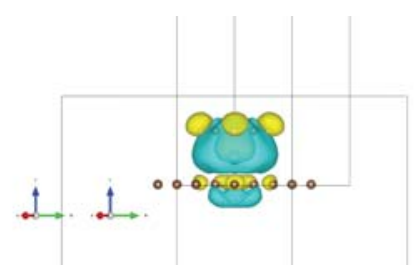

(i) $s$

Figure 7.11: Wannier functions is $\mathrm{Cr}\left(\mathrm{C}_{6} \mathrm{H}_{6}\right)_{2}$, labels under each figure indicate the trial WF. d-orbitals are plotted at $3 \%$ probability p orbitals - at $0.15 \%$ probability

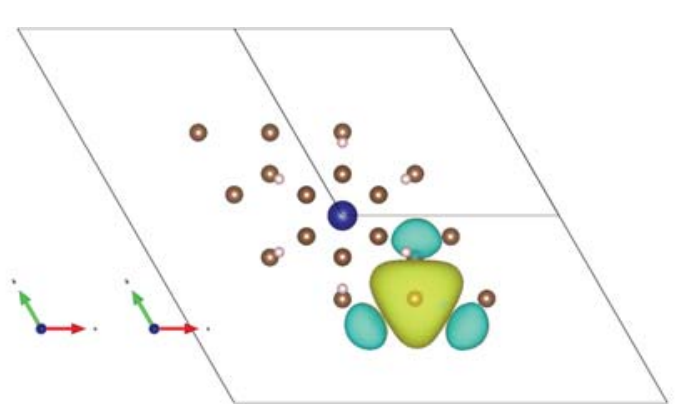

(a) $p_{z}$

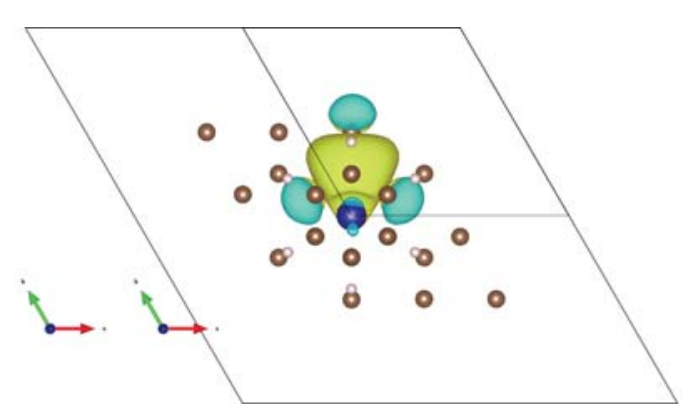

(b) $p_{z}$

Figure 7.12: Wannier functions, centered on carbon atoms far away from the functionalizing metal atom (a), and in the direct vicinity of the metal atom (b).

\subsubsection{Transport in MAFG structures}

Transport properties of MAFG structures are computed in the same manner as it was done for the PFPA structures. However, in case of CrAFG we also studied 
the effect of the degree of functionalization: supercells of different size with single functionalizing molecule per supercell were considered in the same way as it was done in section 7.2. The results of transport computations consist of the standard BCD (bands, conductance, DoS) plots as well as plots comparing ballistic conductance in the Landauer limit (the number of eigenmodes) with the conductance obtained by means of the NEGF formalism. Note that conductance, not conductivity is plotted since comparison with the number of eigenmodes is relevant for conductance, not conductivity. As a way to quantify and compare the results of transport computations for a extensive amount of data, we compared average conductivity in the range $\pm 5 \mathrm{~V}$ relative to the ballistic conductivity of the same compound and relative to pristine graphene. Conductance and conductivity values are summarized in table 7.5 at the end of the chapter.

Transport in CrAFG with various degree of functionalization. As it can be seen in figure 7.13 , functionalization does not alter the conductivity along the $\Gamma-\mathrm{M}$ direction as much as along the $\Gamma-\mathrm{K}$. The reason for this is that even pristine graphene is a semiconductor along $\Gamma-\mathrm{M}$, and therefore functionalization preserves this bands arrangement making electron transport in this direction close to ballistic. Therefore a very good estimate of conductance in this direction can be obtained from the band structure alone and therefore is not included into figure 7.13. Unlike covalent functionalization, flat bands that are associated with d-AOs do not contribute to transport because electron's effective mass $m^{*} \rightarrow \infty$. This consideration is more relevant for practical applications than pure band structure, since I-V curves are obtained by integration of the conductivity curves by means of Eq.(3.16). Therefore band gaps extracted from conduction experiments are generally higher than those formally obtained from band structure. This idea is inherited from consideration of 
shallow dopant levels in conventional semiconductors: band gap there is defines as a property of the host matrix, not the distance between dopant levels.

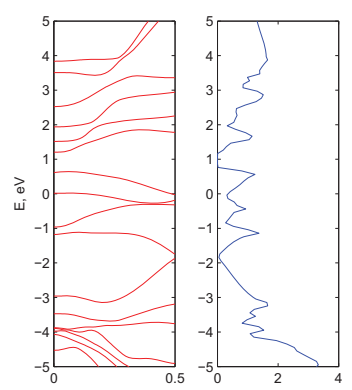

(a) hex22
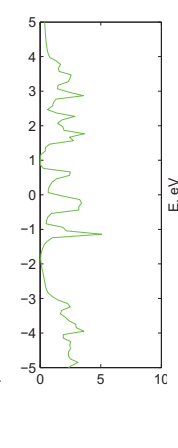

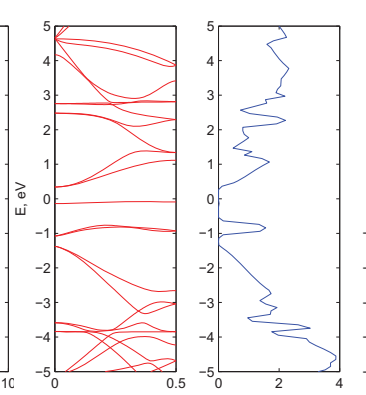

(b) hex33

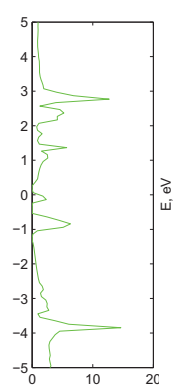

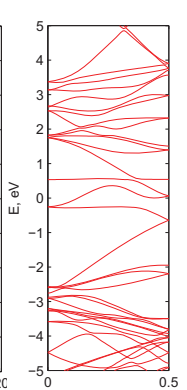

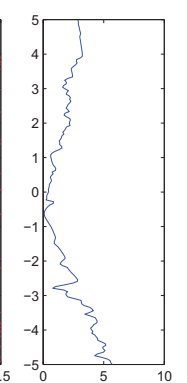

(c) hex44
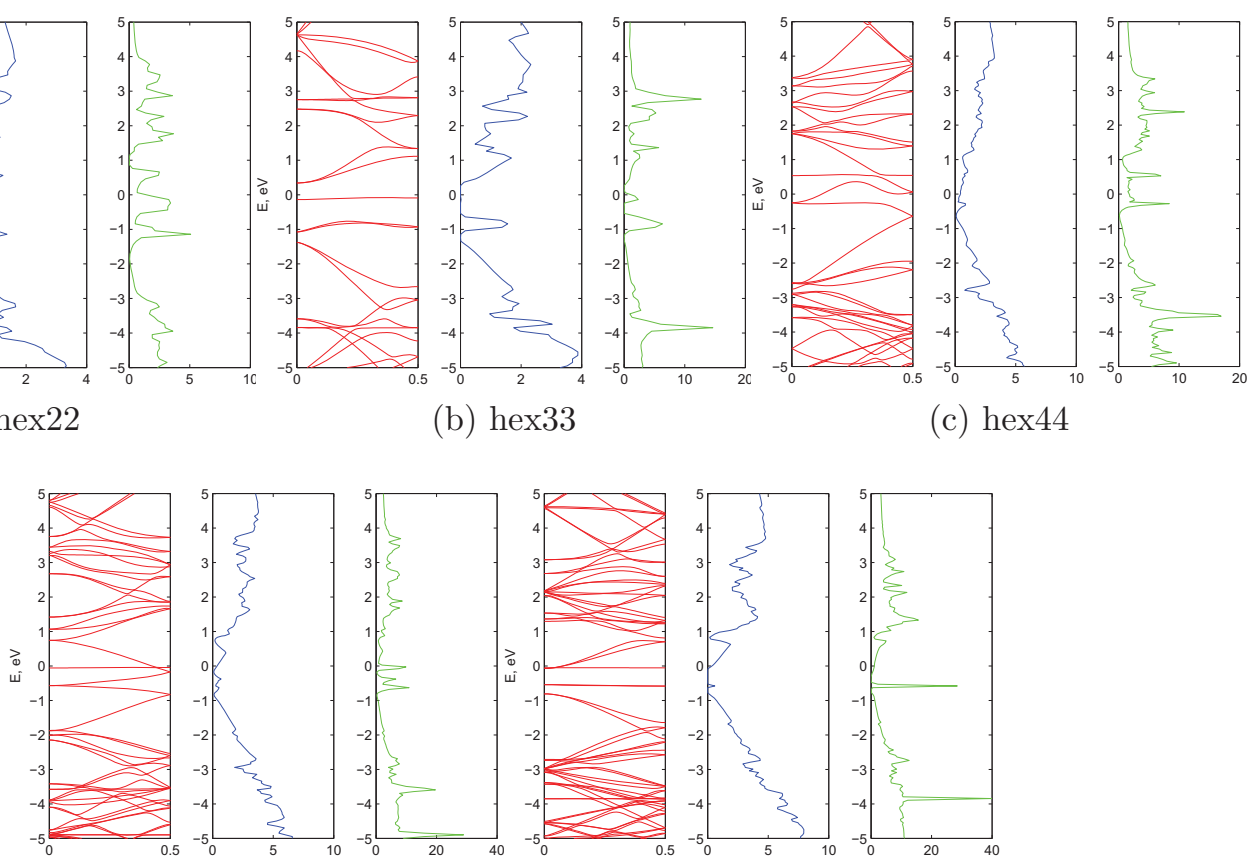

(d) hex 55

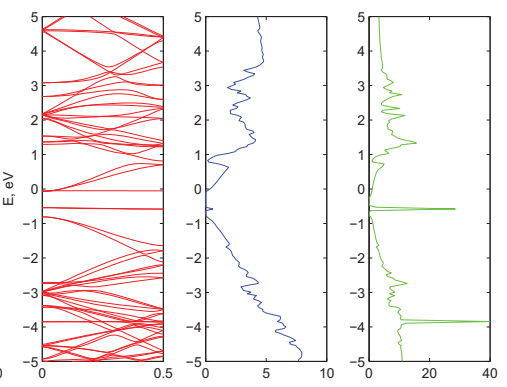

(e) hex66

Figure 7.13: BCD curves for MAGF with different degree of functionalization. Hex88 is missing due to a limitation imposed by the memory volume necessary to store the wave functions.

In contrast to that, the $\Gamma-\mathrm{K}$ direction is far more affected by functionalization especially in the vicinity of the charge neutrality point. As one can see from Fig. 7.13 and Table 7.5. Obviously, as the degree of functionalization decreases, the conductivity recovers to that of pristine graphene. Average conductivities in the range $\pm 5 \mathrm{~V}$ are plotted in figure 7.14 .

Figure 7.14 clearly reveals a declining trend, with the rate of degradation enduring a break at hex33. It is surprising that the highest degree of functionalization (hex22, 2x2 supercell) has the highest conductivity, since in the MAFG structures the band gap increases with increasing degree of functionalization (disregarding of the BZ fold- 


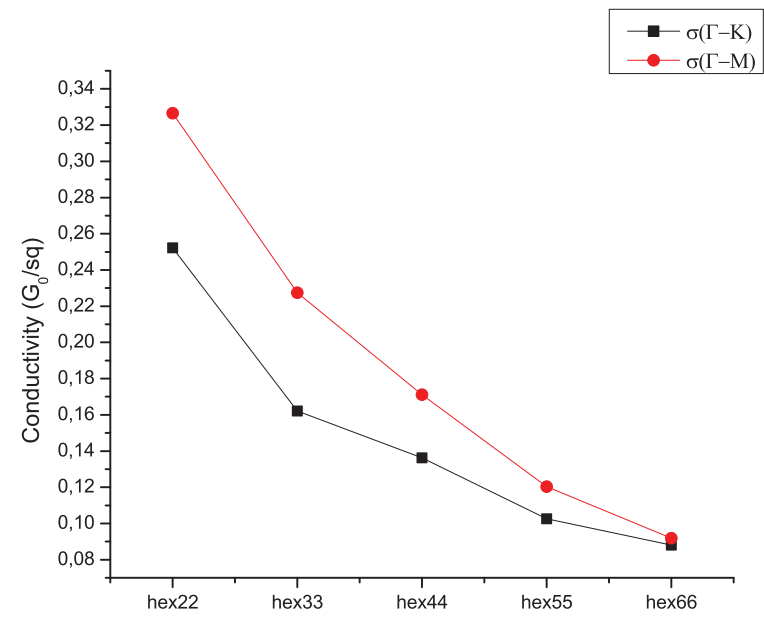

Figure 7.14: Conductivity (in $\frac{e^{2}}{h} / \square$ ) for Cr-AFG with different degrees of functionalization.

ing that obscures this phenomenon). The best results in MA-functionalization of graphene are achieved at the highest possible degree of functionalization. Since the 2x2 structure is already sterically hindered and thus undergoes structural relaxation leading to a parquet pattern arrangement of functionalizing molecules, this is probably the highest possible practically achievable degree of functionailzation. Therefore the synthesis of hex22 should be self-limiting. The break in the curve in Fig. 7.14 is also caused by sterical relaxation of hex33 and further structures, which leads to flatting of the benzene rings with respect to the graphene sheet plane.

Transport in other MAFG structures. Other MAFG structures exhibit in general same the trends, the only difference arises from a different number of valence electrons of the central metal atom, which manifests itself in a different occupation of energy levels (see Fig. 7.4). As it follows from table 7.2, some MAFG structures have an odd number of electrons occupying the HOCO, and therefore those structures will demonstrate intrinsic magnetism and thus should be treated within the spin unrestricted approximation. For 4-V, 4-Mn, and 4-FeAFG, transport was calculated in 
the spin polarized approximation, i.e. with two sets of WF to account for spin up and spin down electrons.

Figures 7.15 demonstrate behavior similar to that previously observed in covalently functionalized graphene. The transport is very close to the ballistic limit in the $\Gamma$-M direction and somewhat preserves the $\mathrm{V}$-shaped graphene conductivity curve in the $\Gamma$-K direction. In 1-MAFG structures as the number of valence electrons increases, the distance between flat $\delta$ and $\delta^{*}$ flat bands also increases, pulling apart graphene $\pi$ and $\pi^{*}$ bands. However, on the other hand, increasing electron count also causes the Fermi level to drift up, so that it may at some point start intersecting the lowest valence bands, causing the structure to become metallic. As the concentration

of functionalizing adducts increases, the interaction between d-electrons belonging the the neighboring metal atoms causes $\delta$ and $\delta^{*}$ bands to repel even more. This enhanced interaction between d-electrons lead to their spin interaction leading to significant energy shift for spin-up and spin-down bands. Band structures for compounds with different central metal atoms having paired electrons obviously do not demonstrate any ferromagnetic behavior.

\subsection{Magnetic properties of MAFG structures}

As it was previously demonstrated, graphene functionalized with transition metal atoms can show profound magnetic properties [147].

Our calculations of band structure for spin up $(|\uparrow\rangle)$ and spin down $(|\downarrow\rangle)$ electrons demonstrate significant degree of polarization in $\mathrm{V}-$, Mn-, and Fe- compounds . Orbital energy splitting is present in 4-MAFG structures and is absent in 1-MAFG. This can be understood from the spatial decay of exchange integral, so that the value of $J_{i j}$ becomes less than thermal smearing at the distance between function- 


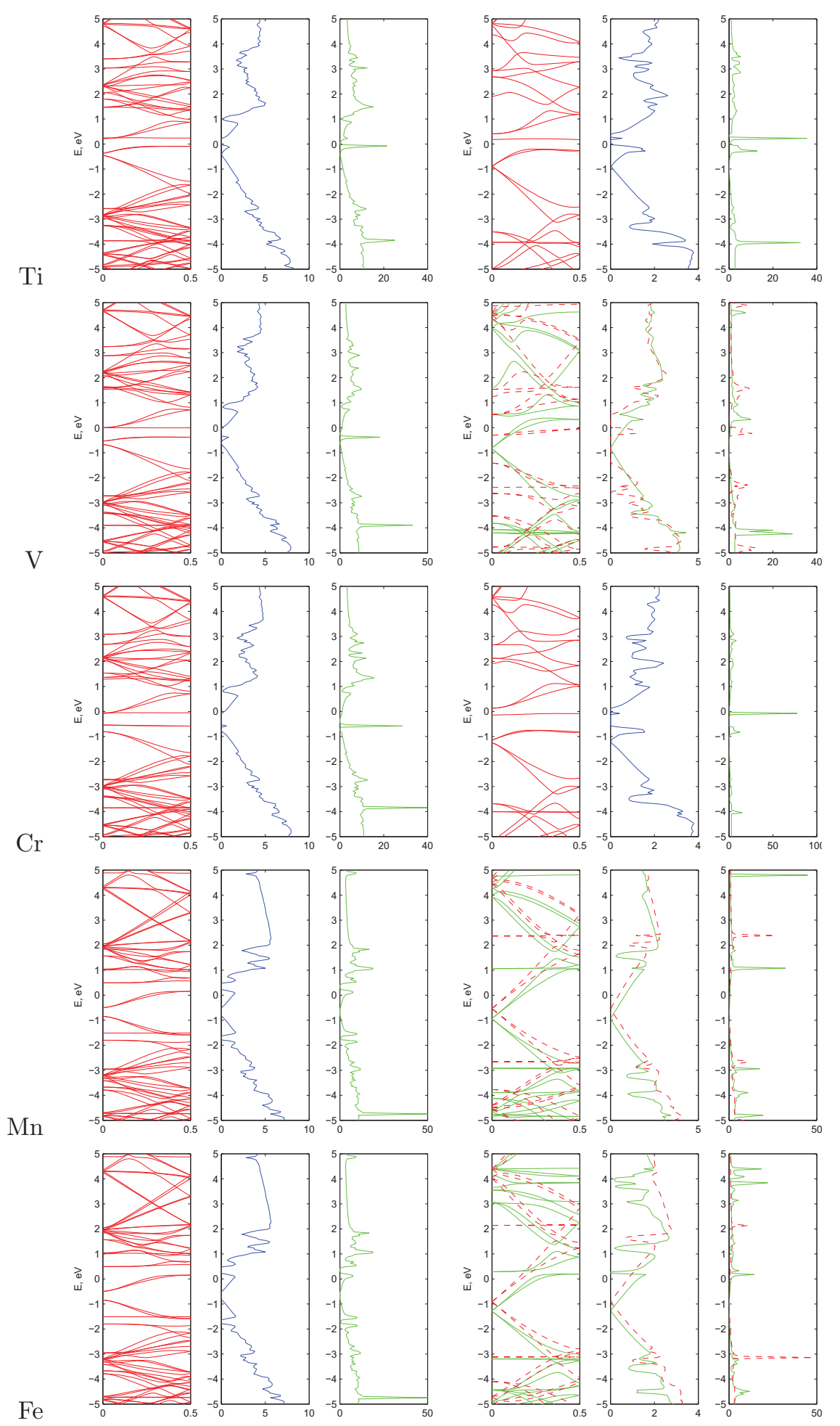

Figure 7.15: Band structure (in $k / k_{\max }$, left panel), conduction (in $\frac{e^{2}}{h}$, middle panel), and density of states (in $\mathrm{eV}^{-1}$, right panel) in the $\Gamma-\mathrm{K}$ direction of 1-MAFG (left column) and 4-MAFG (right column), where $\mathrm{M}=\mathrm{Ti}, \mathrm{V}, \mathrm{Mn}$ (FM), Fe. Red (solid) and blue (dashed) coloring correspond to spin up and spin down bands. 
alizing molecules in 1-MAFG, and in 4-MAFG, however, the distance between the $\mathrm{i}^{\text {th }}$ and the $\mathrm{j}^{\text {th }} \mathrm{d}$-metal atoms is small enough, so that the value of exchange integral $J_{i j} \gg k T$. Thus the interaction between d-electrons of neighboring metal atoms leads to the ferromagnetic spin arrangement.

Spin-unrestricted calculations of 4-VAFG and 4-FeAFG (Figures 7.16a and 7.16b) reveal a significant difference between densities of states (DoS) for spin-up $(\alpha)$ and spin-down $(\beta)$ electrons pointing to ferromagnetic behavior of 4-VAGF and 4-FeAGF. The iron compound demonstrates an even stronger difference in the DoS for the spin-up and spin-down electrons. Total magnetization is calculated from the spinup and spin-down DoS as: $M=\int_{-\infty}^{E_{F}}\left(N_{\uparrow}-N_{\downarrow}\right) d E$, where $N_{\uparrow}\left(N_{\downarrow}\right)$ are integrated DoS of $|\uparrow\rangle$ and $|\downarrow\rangle$ electrons respectively. Magnetization for 4-VAFG and 4-FeAFG is $2.9027 \mu_{B}$ and $1.3894 \mu_{B}$, respectively. Spin density (the difference between $\rho_{\Uparrow \uparrow}$ and $\rho_{\Downarrow \downarrow}$ ) is significantly localized on the metal atoms, as it is to be expected. Due to presence of unpaired electrons, spin polarized structures can exist in both ferromagnetic (FM) and antiferromagnetic (AFM) states.

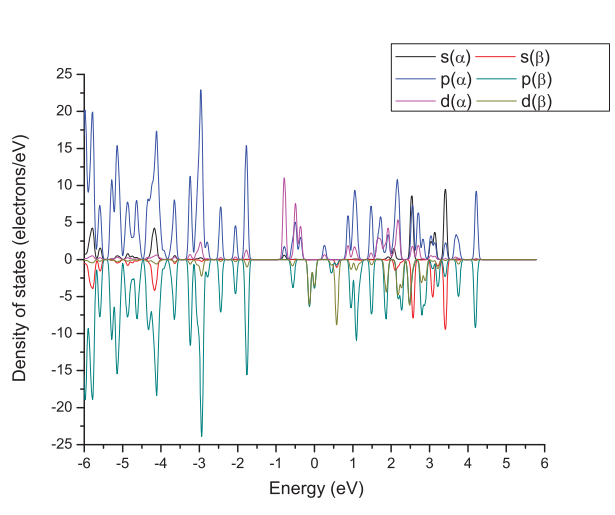

(a)

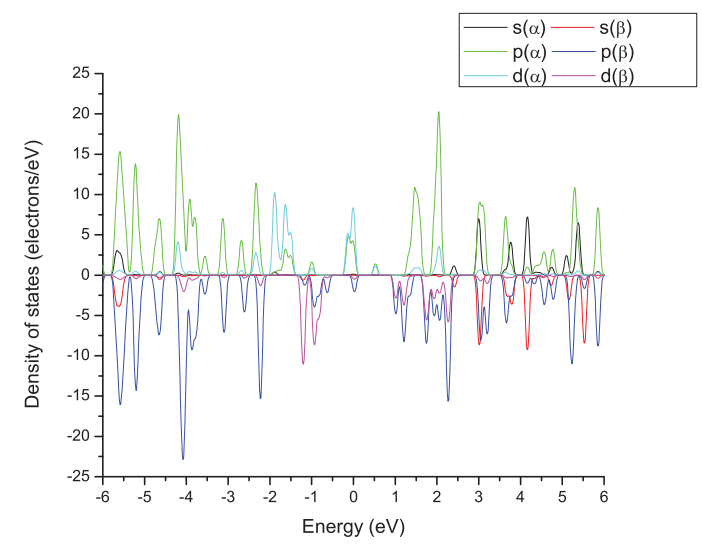

(b)

Figure 7.16: Spin resolved density of states in 4-VAFG (a) and 4-FeAFG (b)

Unlike $\mathrm{V}$ and $\mathrm{Fe}, \mathrm{Mn}$ (in $d^{5}$ ) is known to prefer antiferromagnetic configuration 
(see e.g. [148]), since it possesses one d-electron per each orbital. The AFM ordering is accompanied by doubling of the unit cell by means of the transformation matrix Eq. (4.6) (see Fig. 7.17). Energy difference between the FM (Fig. 7.17(a)) and the AFM (Fig. 7.17(b)) configurations for 4-MnAFG is $\Delta E_{m}=0.87 \mathrm{eV}$ (antiferromagnetic configuration has lower energy) per single metal atom, which suggests that the AFM configuration if much more stable than the FM.

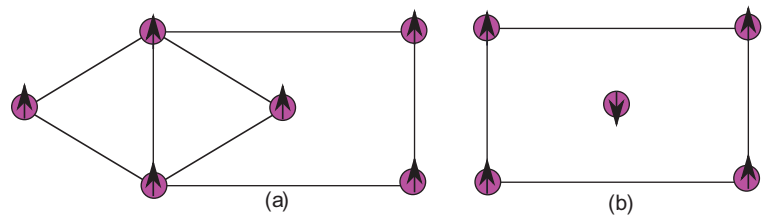

Figure 7.17: Ferromagnetic (a) and antiferromagnetic (b) arrangement of spins of Mn atoms (purple rings)

The FM modification is semiconducting with a direct band gap for spin up electrons of $0.30 \mathrm{eV}$ and an indirect one of $0.36 \mathrm{eV}$ for spin down electrons. The more stable AFM modification is metallic. Thus 4-MnAFG structures can act as electric relays controlled by the local magnetic field.

Transport in FM and AFM modifications of 4-MnAFG. 4-MnAFG structure is of a special interest since it a) is capable for the Jahn-Teller distortion (one electron occupying double degenerate orbitals), that explains unusual behavior of the bond lengths in Figs. 7.8 (a) and (b), and also it is known (see e.g. [149, 150] etc.) that if unpaired electrons are present, Mn often prefers antiferromagnetic (AFM) arrangement to the ferromagnetic (FM) one. Therefore two possible structures were considered for this calculation, corresponding to the sketches in Fig. 7.17. Initial magnetization, i.e. spin for all valence electrons in Mn atoms was set to +1 in the FM modification and to \pm 1 in the alternating fashion in the AFM modification. Final 
magnetization for the structures became ${ }^{4}$ :

Table 7.3: Magnetization values in FM and AFM 4-MnAFG structures

\begin{tabular}{cccc}
\hline & $M_{\text {tot }}\left(\right.$ in $\mu_{B} /$ cell $)$ & $M_{\text {abs }}\left(\right.$ in $\mu_{B} /$ cell $)$ & $M_{\text {tot }}$ (in $\mu_{B} /$ atom $)$ \\
\hline AFM: & 5.18 & 20.14 & 1.295 \\
\hline FM: & 17.46 & 20.14 & 4.365 \\
\hline
\end{tabular}

Wannier functions were computed separately for spin up and spin down electrons.

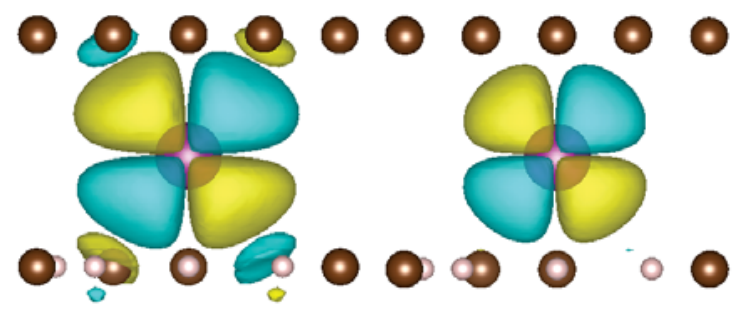

(a) spin up

(b) spin down

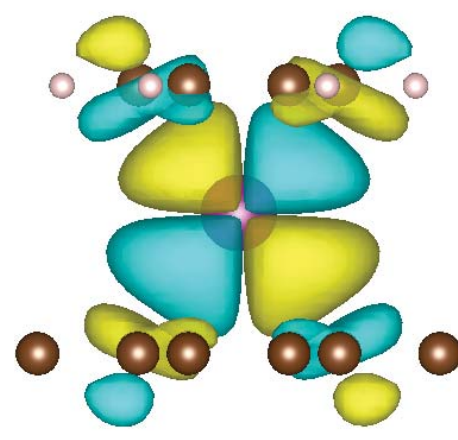

(c) spin up

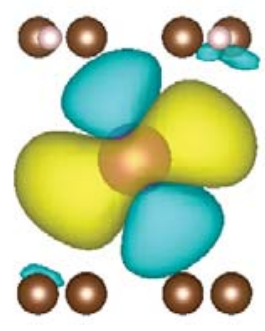

(d) spin down

Figure 7.18: Wannier functions for 4Mn-MAFG, AFM (a,b) and FM (c,d) modifications

In Fig. 7.18(a,b) the WFs for the spin up and spin down electrons in the AFM modification do not differ qualitatively, however, the volume enclosed by spin-up and

\footnotetext{
${ }^{4}$ Total magnetization: $M_{t o t}=\int\left(n_{\uparrow}(\mathbf{r})-n_{\downarrow}(\mathbf{r})\right) d^{3} \mathbf{r}$, Absolute magnetization: $M_{a b s}=$ $\int\left|n_{\uparrow}(\mathbf{r})-n_{\downarrow}(\mathbf{r})\right| d^{3} \mathbf{r}$. For a FM material $M_{t o t}=M_{a b s}$, for an AFM material $M_{t o t}=0, M_{a b s}$ is twice the magnetization of each of the two Mn (in our case) atoms
} 
spin-down isosurfaces of the same isovalue, slightly differs, which suggests that the modification initialized as AFM, actually demonstrates ferrimagnetism, which agrees with the nonzero magnetization levels (Table 7.3) obtained by integration of the DoS. In the FM modification (Fig. 7.18(c,d)) WF, corresponding to the $d_{x z}$ (as well as $d_{y z}$, $\left.d_{z^{2}}\right)$ are inclined with respect to the initial magnetization axis $(z)$. This suggest a nontrivial magnetic structure, like incommensurate spin arrangement or spin waves. This nontrivial magnetic structure would probably explain the difference between $M_{\text {tot }}$ and $M_{a b s}$ in table 7.3. We however, limit our consideration to the commensurate case of checkerboard small cell spin arrangement.

Two separate sets of BCDs are obtained as well. From Fig. 7.19 it can be seen that as expected from paragraph 7.5, presence of occupied states in the vicinity of the Fermi level for spin up electrons supples conduction channels at low bias making the 4Mn-MAFG structure a conductor for spin up electrons. On the other hand, eigenmodes for spin down electrons are absent, therefore in order for this structure to stop discriminating between spin up and spin down electrons a fairly large bias (3V) should be applied.

The conductance in Fig. 7.19 has to be normalized by $9(3 \times 3)$, the size of the supercell for the FM modification) and by $31.17(3 \times 3 \times \sqrt{3} \times 2)$ - supercell for the AFM modification, compared in Fig. 7.17(a) and Fig. 7.17(b).

The charge neutrality point is shifted to $-1 \mathrm{eV}$ for the $|\uparrow\rangle$ and $-0.72 \mathrm{eV}$ for the $|\downarrow\rangle$ electrons in the FM modification, and by $-1 \mathrm{eV}$ and $-1.3 \mathrm{eV}$ for the $\mathrm{AFM}$ modification respectively. That means that $\mathrm{Mn}$ as other MA molecules act as a p-dopant in both magnetic structures.

Therefore as it follows from Fig. 7.19 (middle panels) relative difference between spin up and spin down conductivities $\left(\frac{\left\langle\sigma_{\uparrow}\right\rangle-\left\langle\sigma_{\downarrow}\right\rangle}{\left\langle\sigma_{\uparrow}\right\rangle}\right)$ is about $12 \%$ for the FM modification and, about $10 \%$ for the AFM modification, which is about $20 \%$ lower than 

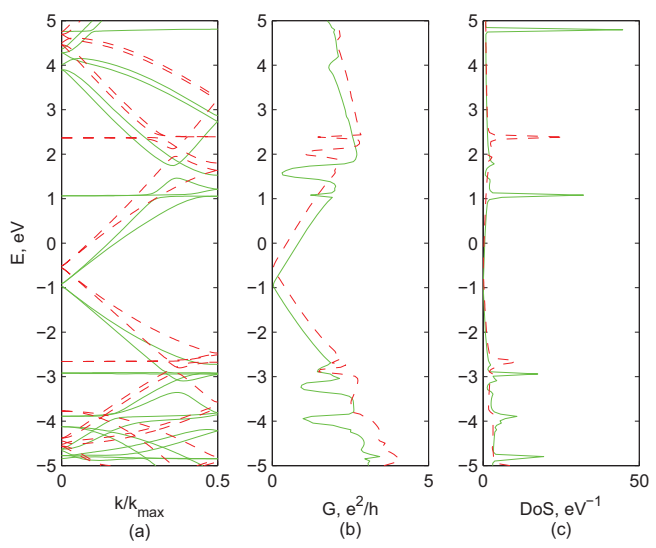

(a) FM
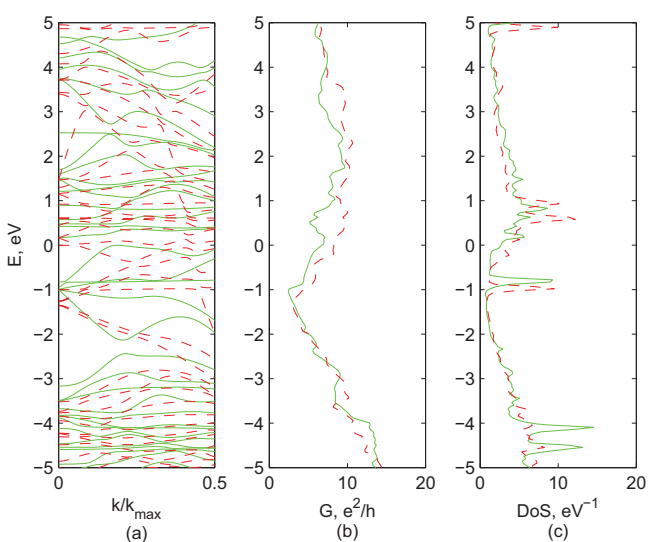

(b) AFM

Figure 7.19: Band structure, conduction, and density of states for spin up electrons (red, solid) and spin down electrons (green, dashed) for the FM (a) and AFM (b) modifications of 4Mn-MAFG. Note that $\Gamma-\mathrm{K}$ direction in the FM cell corresponds to $\Gamma$-X direction in the AFM cell, and also that due to nontrivial BZ folding, band structures do not have one to one correspondence.

the FM modification. This is most likely due to scattering of electrons of different spins on two sublattices (Mn $|\uparrow\rangle$ and Mn $|\downarrow\rangle)$ whereas in the FM modification at least spin up electrons can travel ballistically. Transport in the $\Gamma-\mathrm{M}$ direction is as usual very close to ballistic, at least for the biases of $\pm 3 \mathrm{~V}$.

The presence of nontrivial magnetic structure suggests wide possible applications of the MA functionalized graphene in spintronics, for example as spin-discriminating gates or it can be utilized to detect weak magnetic fields. By creating domains of different magnetization topology, one can efficiently control transport properties of 4-MnAFG.

\section{6 "Piano stool" functionalization}

One disadvantage of the MAFG structures arises from the 18-electron rule. From this rule it follows that structures will loose their stability as the electronic configu- 
ration of the central atoms deviates from $d^{6}$ as it does from the $\mathrm{Cr}^{0}$ atom. However, it is known that uncharged metal complexes are less stable compared to the anionic ones. Therefore to enhance stability of haptically functionalized graphene we need to consider cationic or anionic ligands with the electron count of 6 , same as for the benzene ring. One example of such ligands can be the row $\mathrm{CN}^{-}-\mathrm{CO}^{0}-\mathrm{NO}^{+}$. Charged ligands can accommodate charged metal ions, totaling electron count to 18 . Since each of the ligands has electron count of two, there should be three of them to replace one benzene ring. The structure that is formed by a metal atom sandwiched between three of these ligands and a benzene ring is known as "piano stool" due to its similarity with a three legged stool. (Fig. 7.20) Piano stool functionalization also does not disturb local planarity of graphene sheet as well as MAFG. Therefore almost everything that is said about MAFG can be repeated for Piano stool-FG.

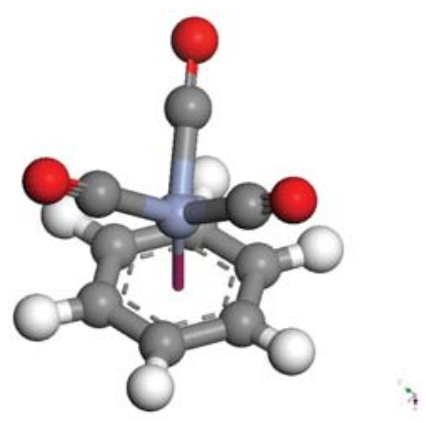

Figure 7.20: Structure of a Chromium(0)-monoarene-tricarbonyl $\left(\mathrm{Cr}\left[\eta^{6}-\left(\mathrm{C}_{6} \mathrm{H}_{6}\right)\right](\mathrm{CO})_{3}\right)$ molecule, an example of a "piano stool"

Interaction between carbonyl groups and d-orbitals of the central metal atom is similar to haptic bonding between d-orbitals and benzene ring. The carbon atom in $\mathrm{CO}$ groups posses one lone pair, that can interact with empty p- or d-orbitals of the metal atom, and empty $\pi^{*}$-orbitals that, in turn, interact filled d-orbitals. The first mechanism leads to a $\sigma$-bonding, the second - to $\pi$-backbonding. Unlike MBA molecules, that do not have an option to bind via $\sigma$ bond, in metal-carbonyl 
(MCO) this type of bonding dominates, leaving d-elections to bind to graphene. This advantage of MCO relative to MBA causes stronger bonding to the substrate.
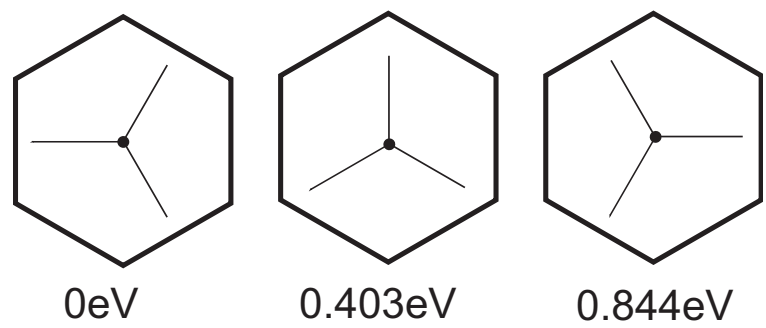

Figure 7.21: Different conformations of MCO molecules and their relative energies.

Strong $\sigma$-bonding also leads to suppression of rotation on the topping groups relative to the lower benzene (graphene) rings. We calculated total energies for MCO molecules in three different conformations (See Fig. 7.21): "eclipsed" (left), "staggered" ("middle"), "reversed eclipsed" (right). Staggered conformation has the lowest energy. Energy barriers of two last conformations relative to the energy of the "eclipsed" conformation are given in figure $7.21^{5}$. One can see that that those energies are significantly higher than that at room temperature $(0.0256 \mathrm{eV})$, therefore we do not expect rotational phenomena to interfere with our further calculation of electronic properties.

\subsubsection{Electronic structure of MCO molecules}

Molecular orbital picture of $\mathrm{M} \longleftarrow \mathrm{CO}$ interaction (Fig. 7.22) suggests that $\pi$ orbitals of carbonyl interact with filled d-orbitals of the metal, and $\sigma$ orbitals of carbonyl interact with empty s- or p-orbitals of the metal. This interaction of three carbonyls (cyanides) forms $\sigma\left(d_{z}+\sigma \longrightarrow 1 a_{1 g}\right), \sigma^{*}\left(p_{z}+\sigma^{*} \longrightarrow 2 a_{1 u}^{*}\right)$, and $\pi^{*}$ $\left.\left(p_{x}\left(p_{y}\right)+\pi^{*} \longrightarrow 2 e_{1}^{*}\right), \pi\left(d_{x y}, d_{x^{2}+y^{2}}\right)+\pi \longrightarrow 1 e_{1}\right)$ orbitals.

\footnotetext{
${ }^{5}$ The energy of the "reversed eclipsed" conformation corresponds to two potential barriers that the $\mathrm{CO}$ moiety groups need to overcome. We do not consider a rigid rotation of the molecule as whole.
} 


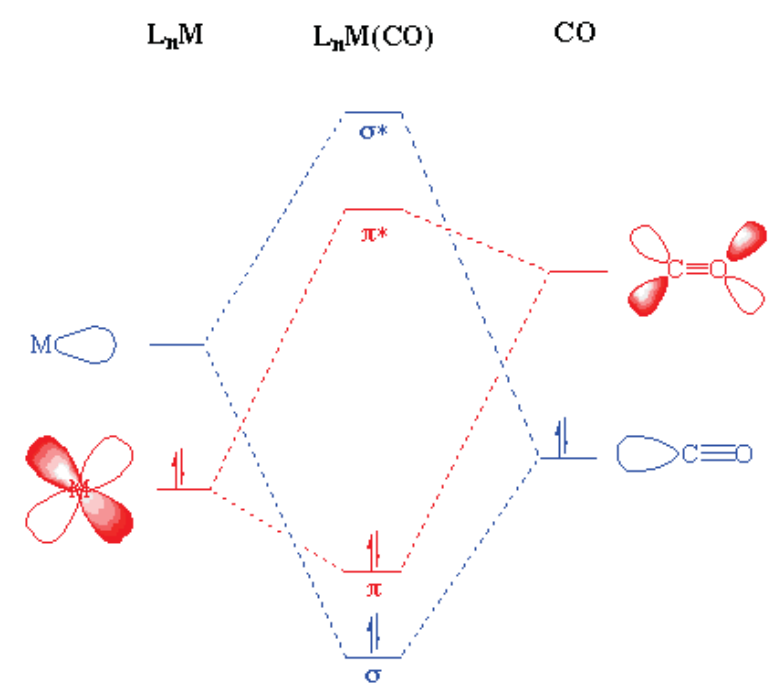

Figure 7.22: Interaction of a $d$-metal with a carbonyl molecule and formation of corresponding molecular orbitals. Adopted from [139].

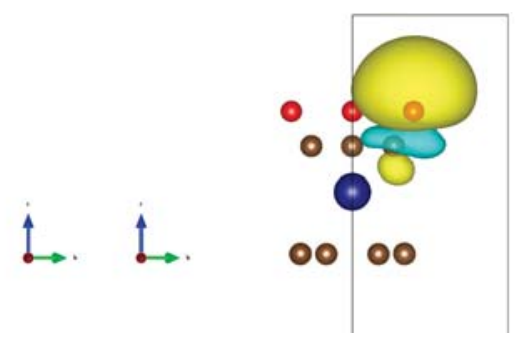

Figure 7.23: WF that corresponds to the lone pairs of carbon and oxygen in $\mathrm{Cr}\left[\eta^{6}-\left(\mathrm{C}_{6} \mathrm{H}_{6}\right)\right](\mathrm{CO})_{3}$ molecule. The LP of the carbon atom is localized stronger since it participates at the bonding process. Corresponding s-, p-, and d-orbitals, responsible for the backbonding process are depicted in Fig. 7.26

The correlation diagram (Fig. 7.22) needs to be overlaid with the MO picture of benzene (Fig. 7.4). The MO diagram in Fig. 7.4 shows various metal-ring interactions: $\sigma\left(\operatorname{Ar}\left(a_{2 u}\right) \longrightarrow \mathrm{Cr}\right), \pi\left(\operatorname{Ar}\left(e_{1 g}\right) \longrightarrow \mathrm{Cr}\right)$ and $\delta\left(\mathrm{Cr} \longrightarrow \operatorname{Ar}\left(e_{2 u}\right)\right)$. The $\sigma$ bond is constructed of $s$ and $d_{z^{2}}$ orbitals of $\mathrm{Cr}(\mathrm{CO})_{3}$ complex interacting with $a_{1 g}$ orbital of benzene, and $\mathrm{Cr}-p_{z}$ interacting with $a_{2 u}$. The HOMO of the complex results in mixture of $a_{1 g}\left(d_{z^{2}}\right)+a_{2 u} \rightarrow a_{1}$ orbitals. The HOMO-1 is constructed from a mixture of antibonding orbitals of benzene and $\operatorname{HOMO}\left(d_{x y}\right.$ and $\left.d_{x^{2}-y^{2}}\right)$ orbitals of $\mathrm{M}(\mathrm{CO})_{3}$ fragment by the equation: $e_{1}\left(d_{x y}, d_{x^{2}-y^{2}}\right)+e_{2 u} \rightarrow e_{2 g}$. The HOMO-2 is created 
by mixing of the $e_{1 g}+2 e\left(p_{x}, p_{y}\right)$. Benzene's $\sigma$-orbitals are preserved in the MCO complexes in form of a non-bonding orbital. The LUMO is constructed from $d_{x z^{-}}$and $d_{y z}$-orbitals by equation: $e_{1}\left(d_{x z}, d_{y z}\right)+e_{1 u} \rightarrow e_{1}^{*}$. From this analysis we conclude that the HOMO/LUMO system primarily consists of d-orbitals of the central atom. Thus as before, we expect the same picture to be preserved in the band structure of periodic Ar-functionalized graphene. Since the LUMO is doubly degenerate, this provides an opportunity for Jahn-Teller distortions, in a very similar way is it happens in MBA complexes.

The same diagram can be used to analyze stability of complexes with different metal atom in the middle. In order to do so we need to consider the 18-electrons rule, but due to the charged ligands the choice possible candidates is significantly wider. We have considered four MCO compounds: $\mathrm{Cr}^{0}\left(\mathrm{C}_{6} \mathrm{H}_{6}\right)(\mathrm{CO})_{3}, \quad \mathrm{Mn}^{0}\left(\mathrm{C}_{6} \mathrm{H}_{6}\right)(\mathrm{CO})_{3}$, $\mathrm{Mn}^{3+}\left(\mathrm{C}_{6} \mathrm{H}_{6}\right)(\mathrm{CN})_{3}$, and $\mathrm{Fe}^{3+}\left(\mathrm{C}_{6} \mathrm{H}_{6}\right)(\mathrm{CN})_{3}$. Usage of isoelectronic cyanide group $\left(\mathrm{CN}^{-}\right)$instead of carbonyl allows to use more stable oxidized forms of metals and maintain electron count close to 18. Local electronic configuration is, again, significantly affected by the electronic structure of the functionalizing metal atom, and especially the different charge transfer due to the ligands other than a benzene ring.

Table 7.4: Electronic configuration of Piano stool-molecules as a function of electron count of the central atom.

\begin{tabular}{ccccccc} 
Molecule & $\mathrm{Cr}(\mathrm{CO})$ & $\mathrm{Mn}(\mathrm{CO})$ & $\mathrm{Cr}(\mathrm{CN})$ & $\mathrm{Mn}(\mathrm{CN})$ & $\mathrm{Fe}(\mathrm{CN})$ & $\mathrm{Co}(\mathrm{CN})$ \\
\hline Electron count & 18 & 19 & 15 & 16 & 17 & 18 \\
$e_{1}\left(x^{2}-y^{2}, x y\right)$ & - & $\uparrow-$ & - & - & - & - \\
$a_{1}\left(z^{2}\right)$ & $\uparrow \downarrow$ & $\uparrow \downarrow$ & - & - & $\uparrow$ & $\uparrow \downarrow$ \\
$e_{1}(x z, y z)$ & $\uparrow \downarrow \uparrow \downarrow$ & $\uparrow \downarrow \uparrow \downarrow$ & $\uparrow \downarrow \uparrow$ & $\uparrow \downarrow \uparrow \downarrow$ & $\uparrow \downarrow \uparrow \downarrow$ & $\uparrow \downarrow \uparrow \downarrow$ \\
\hline $\begin{array}{c}\# \text { of unpaired } \\
\text { electrons }\end{array}$ & 0 & 1 & 1 & 0 & 1 & 0 \\
\hline
\end{tabular}




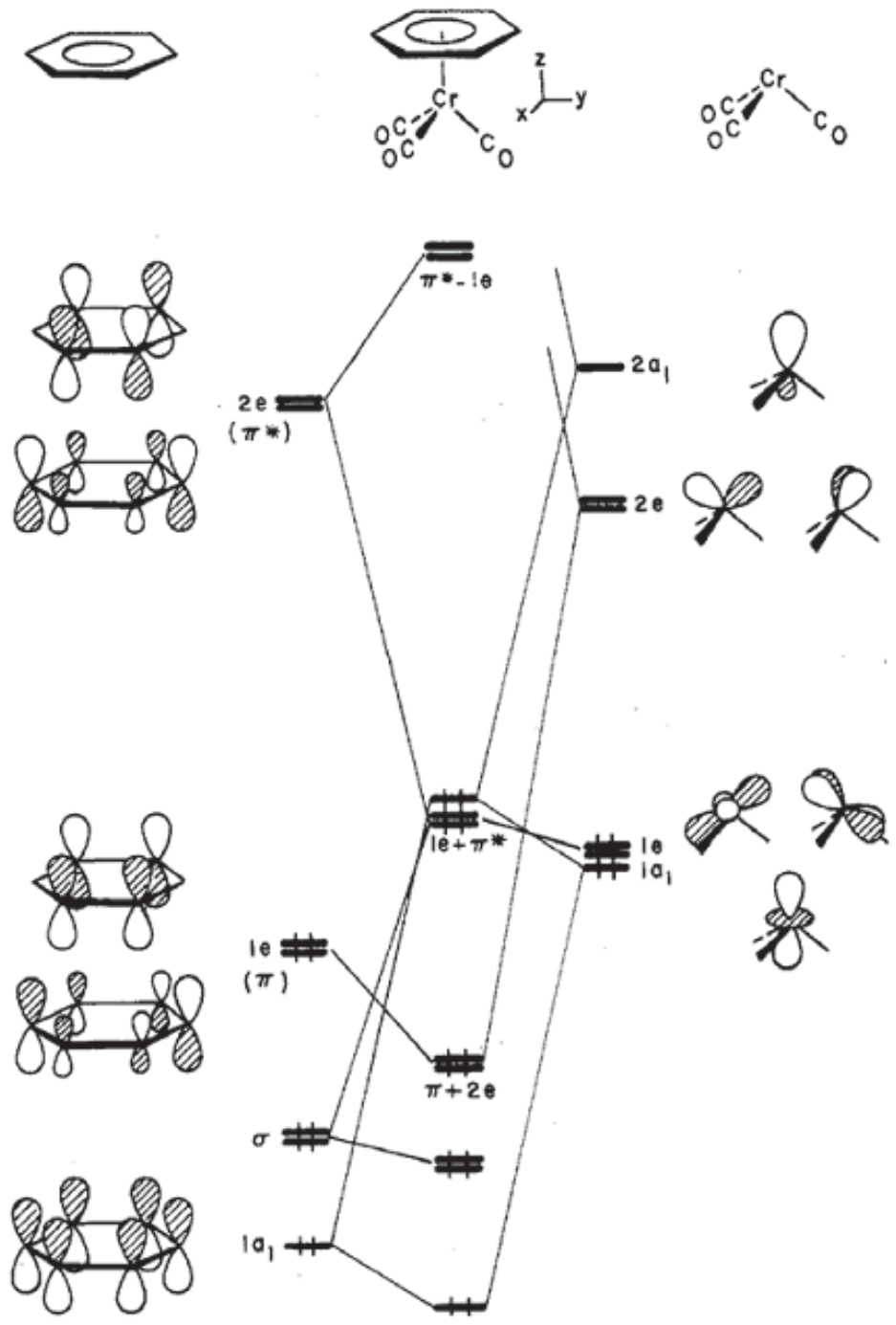

Figure 7.24: Molecular orbital diagram of $\mathrm{Cr}\left(\mathrm{C}_{6} \mathrm{H}_{6}\right)(\mathrm{CO})_{3}$ and interactions of the benzene orbital with the orbitals of the $\mathrm{Cr}(\mathrm{CO})_{3}$ fragment. Adapted from [151]

\subsubsection{Band structure of Piano stool-FG}

For the calculation of the band structure a 4x4 graphene supercell per one functionalizing molecule was chosen. Electron dispersion was calculated along the $\mathrm{M}-\mathrm{\Gamma}-\mathrm{K}$ k-path with 12 and 9 k-points on each segment correspondingly. Due to the supercell geometry (see Sec. 2.6) BZ folding occurs: the Dirac point $K$ of the subcell maps onto $K$ of the supercell, zone center $(\Gamma)$ remains invariant with respect to the folding. 


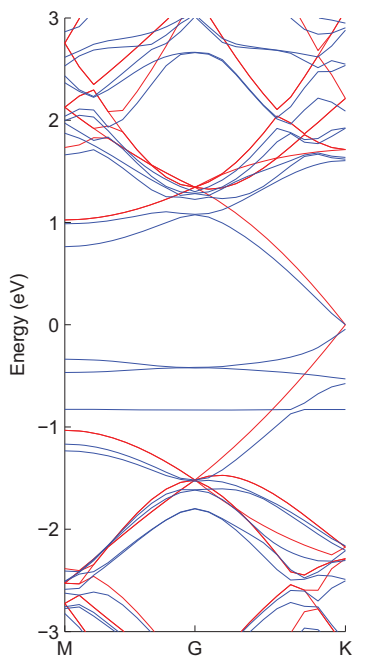

(a) $\mathrm{Cr}\left(\mathrm{C}_{6} \mathrm{H}_{6}\right)(\mathrm{CO})_{3}$

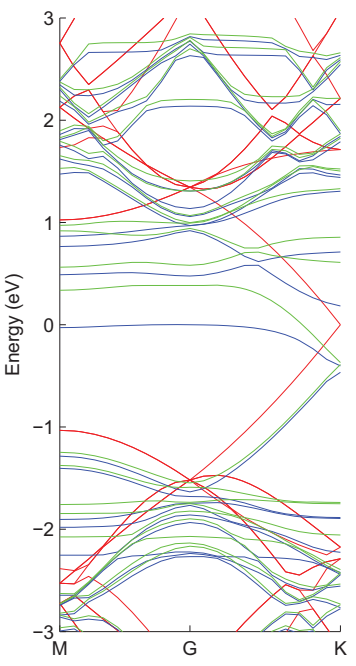

(b) $\mathrm{Mn}\left(\mathrm{C}_{6} \mathrm{H}_{6}\right)(\mathrm{CO})_{3}$

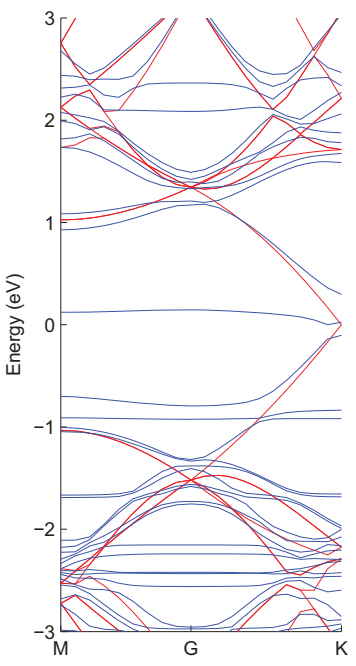

(c) $\mathrm{Mn}\left(\mathrm{C}_{6} \mathrm{H}_{6}\right)(\mathrm{CN})_{3}$

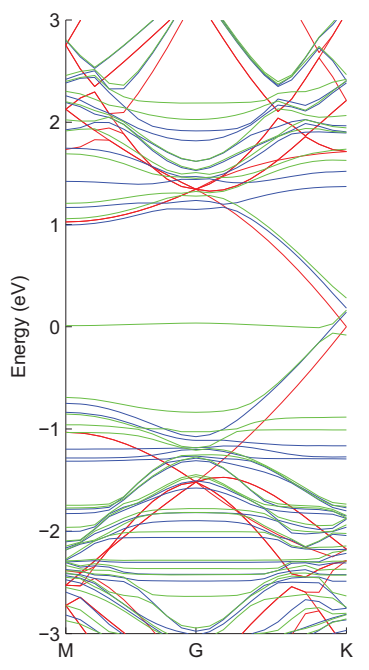

(d) $\mathrm{Fe}\left(\mathrm{C}_{6} \mathrm{H}_{6}\right)(\mathrm{CN})_{3}$

Figure 7.25: Band structures of pristine (red) and Piano stool-functionalized graphene(blue for closed shells or spin up and green for spin down). Band structures were aligned with respect of the position of the Fermi level.

Since MnCO and FeCN structures possess odd number of electrons, spin-orbital interaction leads to band splitting in these substances. The $\mathrm{Mn}\left(\mathrm{C}_{6} \mathrm{H}_{6}\right)(\mathrm{CN})_{3}$ compound can, again, as in the MBA case, exist in two different configurations - FM and AFM ordering, where AFM ordering is, again, accompanied by doubling of the unit cell.

\subsubsection{Wannier functions of PS-FG structures.}

The trial Wannier functions manifold was initialized as usual: graphene subset of $\sigma$ bonds and carbon $p_{z}$ orbitals. The subset of the functionalizing molecule WFs was initialized independently as a set of AOs for the metal atom, i.e. $5 d+3 p+1 s$ orbitals, for $\mathrm{C}$ and $\mathrm{O}(\mathrm{N})$ as $3 p+1 s$. Disentanglement and Wannierization procedures converged rapidly leaving no WF with spread significantly different form the other.

This set of WF was again as in the MAFG complete in terms of representation 


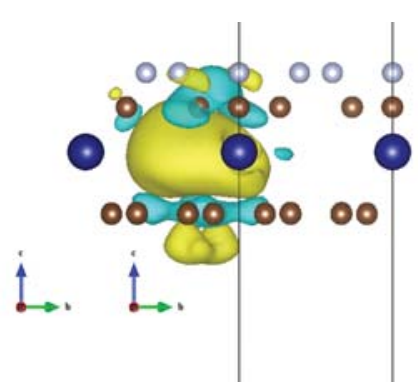

(a) $p_{x}$

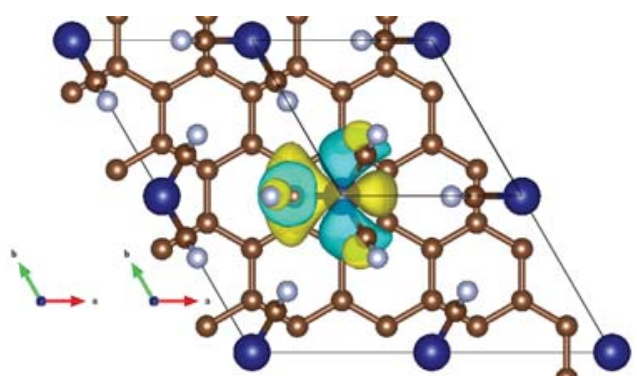

(c) $d_{x^{2}-y^{2}}$

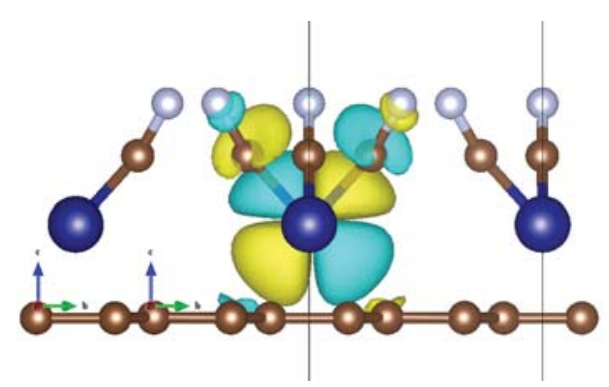

(e) $d_{x z}$

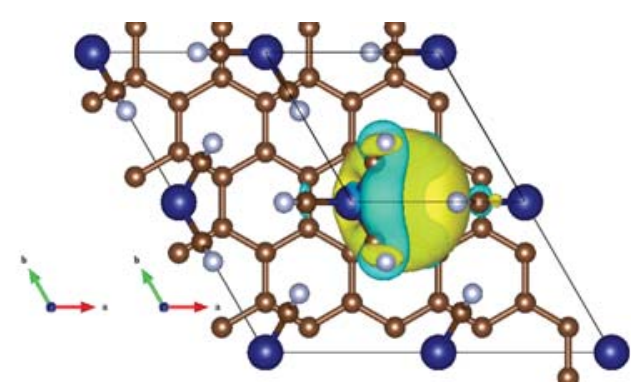

(b) $p_{x}$

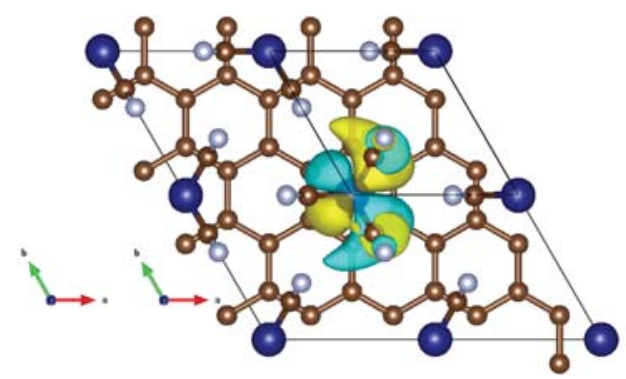

(d) $d_{x y}$

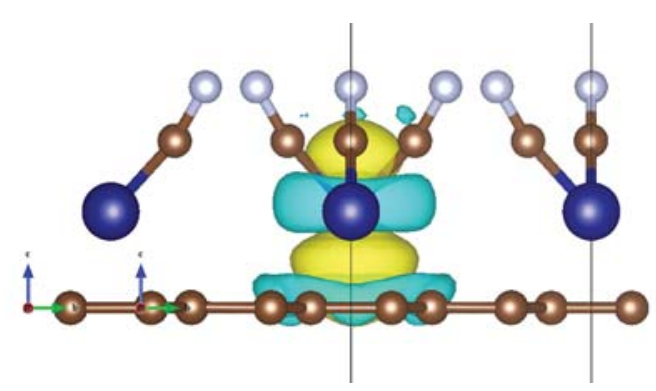

(f) $d_{z^{2}}$

Figure 7.26: Wannier functions is $\operatorname{Cr}\left(\mathrm{C}_{6} \mathrm{H}_{6}\right)(\mathrm{CO})_{3}$, labels under each figure indicate the trial WF.

of Bloch orbitals by Wannier orbitals. Initialized manifold is sufficient to describe transport properties within the energy range of $\pm 5 \mathrm{eV}$. Since $\mathrm{CrCN}$ possesses one unpaired electron, the transport was calculated in the spin polarized approximation, i.e. with two sets of WF for spin up and spin down electrons. We however, present in Fig. 7.26 only the WFs for $\mathrm{CrCO}$, since WFs for CrCN pretty much repeat the same structure with minor difference in localization (see Sec. 7.5). From Fig. 7.26 one can see that the bonding to graphene sheet is provided by the interaction of 
$p_{x}, p_{y}, d_{z^{2}}$ orbitals with $\pi$-system of graphene, where d-orbitals of the metal atom are responsible for bonding with the topping ligands. This is very similar to the bonding picture observed in MBA-structures. Indeed, the energy of the $2 e_{1}$ orbital is much less than that of the $1 e_{1}$ and $1 a_{1}$ (See Figs. 7.4 and 7.24). Unlike MAFG structures binding to graphene occurs in MCO structures via mostly $p$-orbitals, not $\pi-d$ interaction, since those ligands participate in $\sigma$-bonding to lone pairs of the topping ligands.

\subsubsection{Transport in Piano stool-FG structures}

Transport analysis, as always begins with the BCD figures. Comparing band structure in the $\Gamma-\mathrm{K}$ and $\Gamma-\mathrm{M}$ directions for $\mathrm{CrCN}$ and $\mathrm{CrCO}$ with pristine graphene (Fig. 7.13(a) and Fig. 7.5(a)) demonstrates that band alignment is very similar to that of 2-MAFG structures.

From figure 7.27 one can reveal the same trend as for other FG structures: the transport in the $\Gamma$-M direction remains almost ballistic and is governed by the band structure only, but the transport in the $\Gamma$-K direction inherits typical graphene $\mathrm{V}$ shaped behavior near the charge neutrality point. MCO functionalization shifts the charge neutrality point towards negative biases, indicating the fact that $\mathrm{MCO}$ molecules act like p-dopants, however weaker than than MA molecules.

The conductivity of Piano stool-FG structures differs from that in the MAFG structures, with average conductivity in $\pm 5 \mathrm{~V}$ range in 2 - $\mathrm{CrCO}$ which is $3 \%$ less than in 2-CrAFG. The conductivity of $2-\mathrm{CrCN}$ is $17 \%$ higher than $\mathrm{CrCN}$. The reason for this conductivity increase can be linked with the growing polarity of the Cr-CN bond, and thus higher electron density displacement towards topping ligands, and thus Cr atom can now accommodate more electron density donated by graphene sheet, rather than in case of $\mathrm{CrCO}$. Conductance and conductivity values are summarized in table 7.5 


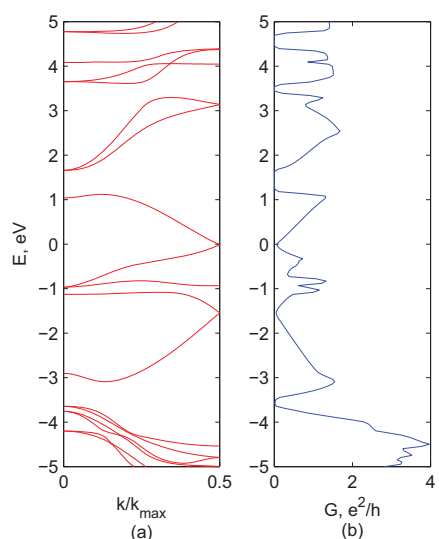

(a) $\mathrm{CrCO} \Gamma-\mathrm{K}$
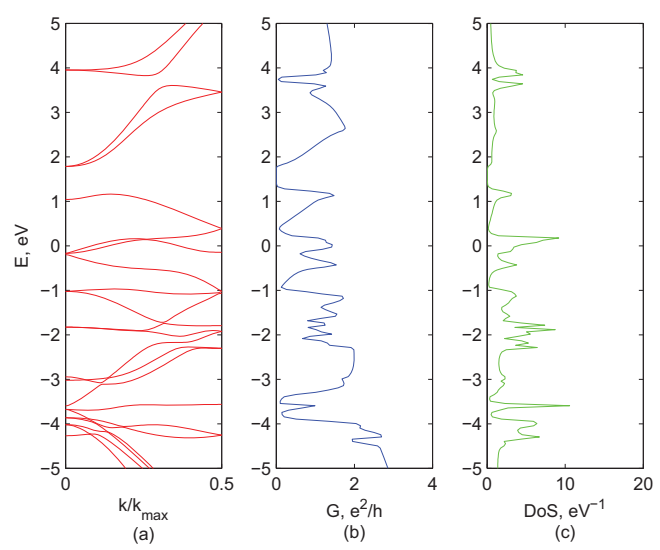

(c) $\mathrm{CrCN} \Gamma-\mathrm{K}$

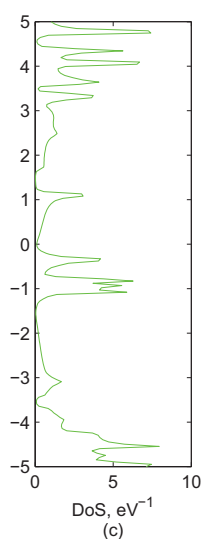

(c)
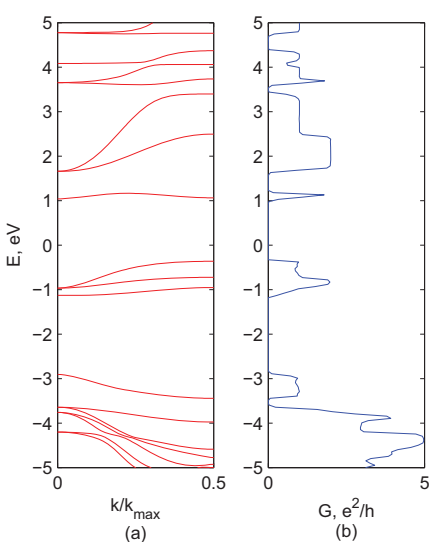

(b) $\mathrm{CrCO} \Gamma-\mathrm{M}$

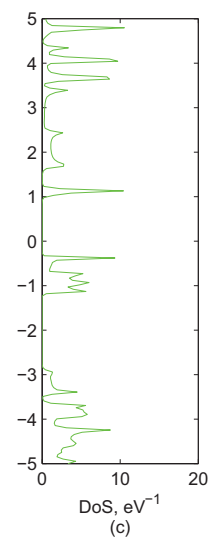

(c)
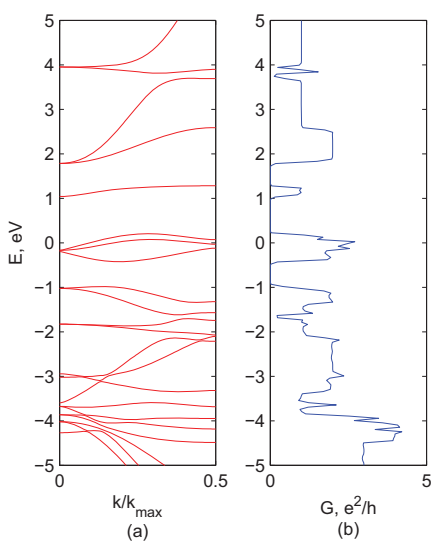

(d) $\mathrm{CrCN} \Gamma-\mathrm{M}$

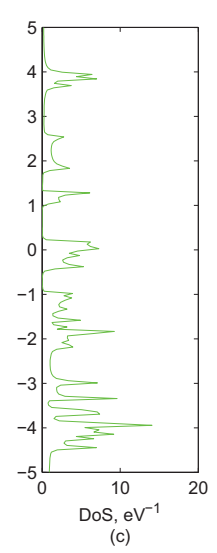

(c)

Figure 7.27: Band structures, conductance, and density of states in two transport directions, $\Gamma-\mathrm{K}$ (left panel) and $\Gamma$-M (right panel) in $\mathrm{Cr}^{0}\left(\mathrm{C}_{6} \mathrm{H}_{6}\right)(\mathrm{CO})_{3}$ (top row) $\mathrm{Cr}^{3+}\left(\mathrm{C}_{6} \mathrm{H}_{6}\right)(\mathrm{CN})_{3}$ (bottom row)

at the end of the chapter.

\subsection{Summary of haptic functionalization of graphene.}

In table 7.5 we summarize numerical results describing electronic properties of MAFG- and Piano stool-FG structures: band gaps, average conductivities, charge transfer, spin, and magnetization. Among all possible modifiers considered in this 
chapter, the highest efficiency is achieved in case of functionalization of graphene by $\mathrm{Cr}(\mathrm{CN})_{3}$ groups. Minimal conductivity degradation is about $60 \%$, and the highest band gap opened in $\mathrm{Cr}(\mathrm{CN})_{3}-\mathrm{FG}$ is more than $1.08 \mathrm{eV}$. This is almost the same value as in silicon - a major material in modern semiconductor industry. Deeper functionalization of graphene sheet with $\mathrm{Cr}(\mathrm{CN})_{3}$ groups can eventually lead to even higher values of gap opening. This suggests a strong support for possible application of functionalized graphene as a replacement of silicon in microchips.

Also it was found that magnetism in MAFG structures is caused by nontrivial spin arrangement, which is interesting from the academic point of view, as well as its potential application in electric switches controlled by a magnetic field. 
Table 7.5: Summary of electronic properties produced by covalently haptically of graphene.

\begin{tabular}{|c|c|c|c|c|c|c|c|}
\hline & Structures & $\begin{array}{r}\text { Band gap } \\
(\mathrm{eV})\end{array}$ & $\begin{array}{r}\left\langle\sigma_{\Gamma-M}\right\rangle \\
\left(\frac{e^{2}}{h} / \square\right)\end{array}$ & $\begin{array}{l}\left\langle\sigma_{\Gamma-K}\right\rangle \\
\left(\frac{e^{2}}{h} / \square\right)\end{array}$ & $\begin{array}{r}\Delta Q \\
(\mathrm{e})\end{array}$ & $\mathrm{S}$ & $\begin{array}{r}\mathrm{M} \\
\left(\mu_{B}\right)\end{array}$ \\
\hline \multicolumn{8}{|l|}{ graphene } \\
\hline Hex & & 0 & 0.7037 & 0.7037 & 0 & & \\
\hline Orth & & 0 & 0.775279 & 0.775279 & 0 & & \\
\hline \multicolumn{8}{|l|}{ 1-MBA } \\
\hline $\operatorname{Ti}\left(\mathrm{C}_{6} \mathrm{H}_{6}\right)$ & & 0.40815 & 0.091613 & 0.099308 & 0.219 & & \\
\hline $\mathrm{V}\left(\mathrm{C}_{6} \mathrm{H}_{6}\right)$ & & 0.10884 & 0.090247 & 0.097946 & 0.051 & & \\
\hline $\mathrm{Cr}\left(\mathrm{C}_{6} \mathrm{H}_{6}\right)$ & & 0.38094 & 0.091181 & 0.099489 & -0.061 & & \\
\hline $\operatorname{Mn}\left(\mathrm{C}_{6} \mathrm{H}_{6}\right)$ & & 0.29931 & 0.092284 & 0.099034 & 0.141 & & \\
\hline $\mathrm{Fe}\left(\mathrm{C}_{6} \mathrm{H}_{6}\right)$ & & 0 & 0 & 0 & 0.022 & & \\
\hline \multicolumn{8}{|l|}{ 4-MBA } \\
\hline $\operatorname{Ti}\left(\mathrm{C}_{6} \mathrm{H}_{6}\right)$ & & 0.32652 & 0.174685 & 0.262337 & 0.526 & & \\
\hline \multirow[t]{3}{*}{$\mathrm{V}\left(\mathrm{C}_{6} \mathrm{H}_{6}\right)$} & FM & & & & 0.343 & 1.427 & 2.708 \\
\hline & $|\uparrow\rangle$ & 0.78909 & 0.219422 & 0.3068 & & & \\
\hline & $|\downarrow\rangle$ & 0 & 0.191713 & 0.243296 & & & \\
\hline $\operatorname{Cr}\left(\mathrm{C}_{6} \mathrm{H}_{6}\right)$ & & 0.8163 & 0.167747 & 0.246373 & 0.107 & & \\
\hline \multirow[t]{3}{*}{$\operatorname{Mn}\left(\mathrm{C}_{6} \mathrm{H}_{6}\right)$} & FM & & & & 0.351 & 3.476 & 4.365 \\
\hline & $|\uparrow\rangle$ & 0.29931 & 0.185067 & 0.263261 & & & \\
\hline & $|\downarrow\rangle$ & 0 & 0.212458 & 0.287134 & & & \\
\hline \multirow[t]{3}{*}{$\operatorname{Mn}\left(\mathrm{C}_{6} \mathrm{H}_{6}\right)$} & $\mathrm{AFM}$ & & & & 0.229 & 3.231 & 1.295 \\
\hline & $|\uparrow\rangle$ & 0 & 0.266871 & 0.078965 & & & \\
\hline & $|\downarrow\rangle$ & 0 & 0.241104 & 0.209816 & & & \\
\hline \multirow[t]{3}{*}{$\mathrm{Fe}\left(\mathrm{C}_{6} \mathrm{H}_{6}\right)$} & FM & & & & 0.297 & 1.496 & 1.791 \\
\hline & $|\uparrow\rangle$ & 0.48978 & 0.155894 & 0.235865 & & & \\
\hline & $|\downarrow\rangle$ & 0 & 0.194431 & 0.272034 & & & \\
\hline \multicolumn{8}{|l|}{$\mathrm{MCO}$} \\
\hline $\mathrm{Cr}(\mathrm{CO})_{3}$ & & 1.0884 & 0.244529 & 0.247226 & -0.203 & & \\
\hline $\mathrm{Cr}(\mathrm{CN})_{3}$ & & 0.24489 & 0.295147 & 0.33556 & -0.654 & 2.412 & 2.9833 \\
\hline $\mathrm{Mn}(\mathrm{CO})_{3}$ & & 0.29931 & & & 0.013 & 0.57 & 0.056 \\
\hline $\mathrm{Mn}(\mathrm{CN})_{3}$ & & 0.78909 & & & 0.247 & & \\
\hline $\mathrm{Fe}(\mathrm{CN})_{3}$ & & 0.68025 & & & 0.36 & 0.933 & 0.8487 \\
\hline $\mathrm{Co}(\mathrm{CN})_{3}$ & & 0.19047 & & & -0.648 & & \\
\hline
\end{tabular}


CHAPTER VIII

Conclusion 


\subsection{Conclusion}

In this work various theoretical properties of pristine and functionalized graphene have been discussed. First few chapters deal with the underlying theory that was used to obtain results presented in the later chapters. The analysis and outline of the main theoretical method used throughout the whole dissertation, the Density Functional Theory, is presented in chapter II. It is important to note that computations, presented in subsequent chapters, were reformed within the framework of this theory. In chapter II we also discuss the necessity of careful analysis and convergence studies (which are often omitted) and possible obstacles on the way to trustful interpretation of the theoretical results. Also the material summarized in chapter III is important to understand the mechanism of charge transport in nanostructures. In chapter IV we discuss experimental work targeting the possibility to readily observe single and multiple layers of graphene, along with simulations of HRTEM contrast from various graphenelike structures. Also in that chapter we state the ability of observe functionalizaing molecules on top of graphene surface by means of high resolution transmission electron microscopy using an aberration-corrected microscope. Visual control of the structure of functionalized graphene is an important step towards experimental verification of the theoretical results presented in the next chapters.

\subsubsection{Pristine graphene}

In chapter $\mathrm{V}$ we not only present calculations, important to compare electronic properties of functionalized graphene with that of pristine one, but also discuss thermal conductivity of graphene nanoribbon. It was found that the difference in thermal conductivity of nanoribbons of different chirality is solely governed by the processes

of edge scattering. We have applied the method of surface roughness function to 
atomistically terminated GNR boundaries. The resulting Green's function and transmission function obtained lead to a thermal conductivity vs. temperature ( $\kappa$-T curve) dependence which is in very close agreement with experimental results.

Also in this chapter we demonstrate how to calculate band structure of graphene for benchmarking and didactic purpose as well as present practical implementation of the MLWF formalism for calculation of quantum transport in pristine graphene. The MLWF formalism is widely used in later chapters to calculate and interpret the effects of functionalization on the electronic structure of graphene.

The explanation of the nature of opening a gap in the band structure of gra-

phene by any functionalization is provided in chapter $\mathrm{V}$, which is very important to understand mechanisms of graphene functionalization presented in chapters VI and VII.

\subsubsection{Covalent functionalization of graphene.}

In chapter VI we discuss the impact of covalent functionalization of graphene surface. Possible candidates considered include epoxy-, nitreno-, and PFPA- functionalized of graphene. This choice fully covers the whole set of nonmetallic atoms that can covalently bind to graphene.

If was found that the band gaps imposed by epoxy- and nitreno- functionalization are not enough to supply feasible $\mathrm{ON} / \mathrm{OFF}$ ratio, as required for a semiconductor transistor. However, detailed analysis of disturbance of conjugated electronic system of graphene by those modifiers reveals relevant features of functionalization, such as importance of the charge transfer and donor/acceptor properties of the modifier. Their relationship with the degree of hybridization and orbital mixing is also examined. This eventually opens the way towards explanation of the effectiveness of PFPA functionalization of graphene. We show in chapter VI that functionalization of gra- 
phene with perfluorophenylazide molecules not only creates a practically usable band gap, but also demonstrates electronic transport properties somewhat close to those of pristine graphene. Conductivity degradation in PFPA structures remains about $50 \%$ relative to nonfunctionalized graphene, however the widest band gap opened in N-PFPA-FG is $0.24 \mathrm{eV}$. This is about $100 \mathrm{kT}$ at room temperature and is already somewhat feasible for microelectronic applications.

\subsubsection{Haptic functionalization of graphene.}

In chapter VII we discuss the impact of haptic functionalization of graphene surface. The importance of haptic functionalization of graphene comes from several major aspects. Since haptic bonding is provided by d-electrons the number of possible functionalizing metal-organic molecules is virtually unlimited, providing broad possibilities for fine tuning of the band structure of hatically functionalized graphene. Second, in contrast to covalent functionalization, haptic functionalizatioin is not accompanied by rehybridization of graphene carbon atoms. That means that electrons traversing through hapticly functionalized graphene sheet will experience less electron-phonon scattering, which comes from deplanarization of graphene sheet.

In chapter VII we consider two different families of molecules: metal-bis-arene and metal-arene-tricarbonyl functionalization. Studies of conductivity as a function of the degree of functionalization reveal declining trend as the functionalization increases. The reason for that the trade off between patterned arrangement of scattering centers (i.e. the degree of ballisticy) vs. the concentration of scattering centers. It appears that one $\mathrm{Cr}\left(\mathrm{C}_{6} \mathrm{H}_{6}\right)$ group per $2 \times 2$ graphene supercell possesses the highest degree of ballisticy. However, the band gap in this compound is not wide enough. Further studies on the dependence of the band gap on the degree of functionalization show that the highest band gap is achieved in 4-MAFG compounds. Fine tuning of the band 
structure can be performed by means of alteration of the electronic structure of the the central metal atom. We analyzed conductivity for multiple 3d-metal functionalized graphene structures. Their band structures and conductivity curves are presented in figures 7.9 and 7.15 and summarized in table 7.5. It was found that linear increase in the number of valence electrons sometimes causes dramatic and nontrivial alternation of the electronic structure of functionalized graphene, however, all the time causes a gap opening.

The metal-carbonyl functionalized structures demonstrate even stronger interaction with graphene sheet leading to a band gap of more than $1 \mathrm{eV}$ wide. The transport in carbonyl functionalized graphene is not significantly impeded by interaction of the d-electrons with the conjugated $\pi$-system of graphene, yielding average conductivity on the same level as metal-bis-arene functionalized graphene.

Research presented in this work resulted in several new peer-reviewed publications on graphene-based materials $[152,153]$, each of which constitutes some part of this dissertation, one invited and two oral talks.

\subsection{Further work}

Most of the results presented in this dissertation are dedicated to "screening" and establishing the trends that will blaze the trail for further research. Further, one should focus on only one class of substances, probably the MBA, and conduct a thorough research of their electric properties and carefully compare them with the experiment.

Among the hypothesis that should be tested are: convergence with respect to the axial supercell size, calculation of the band structure using the GW approximation, fitting of the band structure obtained by the GW method to the LDA+U $(G G A+U)$ 
and evaluating the Hubbard electron repulsion parameter (U) for all metals under consideration.

Besides that, the list of metals considered can be expanded towards $4 \mathrm{~d}$ and $5 \mathrm{~d}$ metals, for they are known to form stable haptic complexes as well.

Delocalized $\pi$-conjugated system of the top benzene ring can act as an efficient electron donor or acceptor, attracting small molecules, which would change the electronic states and affect the conductivity. That can be utilized in the studies of the gas sensing properties of these systems, which are very promising as well. 


\section{BIBLIOGRAPHY}




\section{BIBLIOGRAPHY}

[1] T. Abe and S. Handotai, "A history and future of silicon crystal growth," in Silicon materials science and technology: proceedings of the Eighth International Symposium on Silicon Materials Science and Technology. Electrochemical Society, 1998, p. 157. [Online]. Available: http://books.google.com/books?id=cr8FPGkiRS0C

[2] G. Moore, "Cramming more components onto integrated circuits, Reprinted from Electronics, volume 38, number 8, April 19, 1965, pp. 114 ff." Solid-State Circuits Newsletter, IEEE, vol. 20, no. 3, pp. 33-35, Sep. 2006. [Online]. Available: http://ieeexplore.ieee.org/xpls/abs_all.jsp?arnumber $=4785860$

[3] R. Krithivasan, J. Cressler, M. Khater, D. Ahlgren, and G. Freeman, "Half-terahertz operation of SiGe HBTs," IEEE Electron Device Letters, vol. 27, no. 7, pp. 567-569, Jul. 2006. [Online]. Available: http://ieeexplore.ieee.org/lpdocs/epic03/wrapper.htm?arnumber=1644829

[4] J.-P. Colinge, C.-W. Lee, A. Afzalian, N. D. Akhavan, R. Yan, I. Ferain, P. Razavi, B. O'Neill, A. Blake, M. White, A.-M. Kelleher, B. McCarthy, and R. Murphy, "Nanowire transistors without junctions." Nature nanotechnology, vol. 5, no. 3, pp. 225-9, Mar. 2010. [Online]. Available: http://www.ncbi.nlm.nih.gov/pubmed/20173755

[5] X. Lu and Z. Chen, "Curved pi-conjugation, aromaticity, and the related chemistry of small fullerenes (i C60) and single-walled carbon nanotubes." Chemical reviews, vol. 105, no. 10, pp. 3643-96, Oct. 2005. [Online]. Available: http://www.ncbi.nlm.nih.gov/pubmed/16218563

[6] P. Bai, E. Li, K. T. Lam, O. Kurniawan, and W. S. Koh, "Carbon nanotube Schottky diode: an atomic perspective." Nanotechnology, vol. 19, no. 11, p. 115203, Mar. 2008. [Online]. Available: http://www.ncbi.nlm.nih.gov/pubmed/21730550

[7] A. K. Geim and K. S. Novoselov, "The rise of graphene." Nature materials, vol. 6, no. 3, pp. 183-91, Mar. 2007. [Online]. Available: http://www.ncbi.nlm.nih.gov/pubmed/17330084 
[8] P. Wallace, "The Band Theory of Graphite," Physical Review, vol. 71, no. 9, pp. 622-634, May 1947. [Online]. Available: http://link.aps.org/doi/10.1103/PhysRev.71.622

[9] G. Ruess and F. Vogt, "Höchstlamellarer Kohlenstoff aus Graphitoxyhydroxyd." Monatshefte für Chemie, vol. 78, no. 3-4, pp. 222-242, 1948. [Online]. Available: http://www.springerlink.com/index/n9151h83x2147j33.pdf http://www.springerlink.com/index/10.1007/BF01141527

[10] E. Fradkin, "Critical behavior of disordered degenerate semiconductors," Phys. Rev. B, vol. 33, pp. 3263-3268, 1986.

[11] L. Landau, E. Lifshits, and L. Pitaevskii, Statistical physics. Butterworth-Heinemann, 1980, no. v. 2. [Online]. Available: http://books.google.com/books?id=NaB7oAkon9MC

[12] N. Mermin, "Crystalline order in two dimensions," Physical Review, vol. 176, no. 1, p. 250, 1968. [Online]. Available: http://prola.aps.org/abstract/PR/v176/i1/p250_1

[13] J. Evans, P. Thiel, and M. Bartelt, "Morphological evolution during epitaxial thin film growth: Formation of 2D islands and 3D mounds," Surface Science Reports, vol. 61, no. 1-2, pp. 1-128, Apr. 2006. [Online]. Available: http://linkinghub.elsevier.com/retrieve/pii/S0167572906000021

[14] K. Novoselov, A. Geim, S. Morozov, D. Jiang, M. Grigorieva, S. Dubonos, and A. Firsov, "Two-dimensional gas of massless Dirac fermions in graphene," Nature, vol. 438, no. 7065, pp. 197-200, 2005. [Online]. Available: http://www.nature.com/nature/journal/v438/n7065/abs/nature04233.html

[15] K. S. Novoselov, Z. Jiang, Y. Zhang, S. V. Morozov, H. L. Stormer, U. Zeitler, J. C. Maan, G. S. Boebinger, P. Kim, and A. K. Geim, "Room-temperature quantum Hall effect in graphene." Science (New York, N.Y.), vol. 315, no. 5817, p. 1379, Mar. 2007. [Online]. Available: http://www.ncbi.nlm.nih.gov/pubmed/17303717

[16] F. Schedin, A. Geim, S. Morozov, E. W. Hill, P. Blake, M. Katsnelson, and K. Novoselov, "Detection of individual gas molecules adsorbed on graphene." Nature materials, vol. 6, no. 9, pp. 652-5, Sep. 2007. [Online]. Available: http://www.ncbi.nlm.nih.gov/pubmed/17660825

[17] V. M. Pereira, A. H. Castro Neto, and N. M. R. Peres, "Tight-binding approach to uniaxial strain in graphene," Physical Review B, vol. 80, no. 4, p. 45401, 2009.

[18] B. Trauzettel, D. V. Bulaev, D. Loss, and G. Burkard, "Spin qubits in graphene quantum dots," Nature Phys., vol. 3, p. 192196, 2007. 
[19] K. Nakada, M. Fujita, G. Dresselhaus, and M. Dresselhaus, "Edge state in graphene ribbons: Nanometer size effect and edge shape dependence," Physical Review B, vol. 54, no. 24, pp. 17954-17961, Dec. 1996. [Online]. Available: http://link.aps.org/doi/10.1103/PhysRevB.54.17954

[20] L. Brey and H. Fertig, "Electronic states of graphene nanoribbons studied with the Dirac equation," Physical Review B, vol. 73, no. 23, p. 235411, Jun. 2006. [Online]. Available: http://prb.aps.org/abstract/PRB/v73/i23/e235411

[21] K. A. Ritter and J. W. Lyding, "The influence of edge structure on the electronic properties of graphene quantum dots and nanoribbons." Nature materials, vol. 8, no. 3, pp. 235-42, Mar. 2009. [Online]. Available: http://www.ncbi.nlm.nih.gov/pubmed/19219032

[22] X. Li, X. Wang, L. Zhang, S. Lee, and H. Dai, "Chemically Derived, Ultrasmooth Graphene Nanoribbon Semiconductors," Science, vol. 319, no. 5867, pp. 1229-1232, 2008.

[23] K. Subrahmanyam, A. Ghosh, A. Gomathi, A. Govindaraj, and C. Rao, "Covalent and Noncovalent Functionalization and Solubilization of Graphene," Nanoscience and Nanotechnology Letters, vol. 1, no. 1, pp. 28-31, 2009.

[24] S. Patchkovskii, J. S. Tse, S. N. Yurchenko, L. Zhechkov, T. Heine, and G. Seifert, "Graphene nanostructures as tunable storage media for molecular hydrogen." Proceedings of the National Academy of Sciences of the United States of America, vol. 102, no. 30, pp. 10 439-44, Jul. 2005. [Online]. Available: http://www.pubmedcentral.nih.gov/articlerender.fcgi?artid=1180759

[25] C. Ataca, E. Akturk, S. Ciraci, and H. Ustunel, "High-capacity hydrogen storage by metallized graphene," Applied Physics Letters, vol. 93, no. 4, p. 043123, 2008. [Online]. Available: http://link.aip.org/link/APPLAB/v93/i4/p043123/s1\&Agg=doi

[26] K. Persson, V. A. Sethuraman, L. J. Hardwick, Y. Hinuma, Y. S. Meng, A. van der Ven, V. Srinivasan, R. Kostecki, and G. Ceder, "Lithium Diffusion in Graphitic Carbon," The Journal of Physical Chemistry Letters, vol. 1, no. 8, pp. 1176-1180, Apr. 2010. [Online]. Available: http://pubs.acs.org/doi/abs/10.1021/jz100188d

[27] M. Rubes, P. Nachtigall, J. Vondrasek, and O. Bludsky, "Structure and Stability of the WaterGraphite Complexes," The Journal of Physical Chemistry $C$, vol. 113, no. 19, pp. 8412-8419, May 2009. [Online]. Available: http://pubs.acs.org/doi/abs/10.1021/jp901410m 
[28] Z.-B. Liu, Y.-F. Xu, X.-Y. Zhang, X.-L. Zhang, Y.-S. Chen, and J.-G. Tian, "Porphyrin and fullerene covalently functionalized graphene hybrid materials with large nonlinear optical properties." The journal of physical chemistry. B, vol. 113, no. 29, pp. 9681-6, Jul. 2009. [Online]. Available: http://www.ncbi.nlm.nih.gov/pubmed/19569625

[29] J. Choi, K.-j. Kim, B. Kim, H. Lee, and S. Kim, "Covalent Functionalization of Epitaxial Graphene by Azidotrimethylsilane," The Journal of Physical Chemistry C, vol. 113, no. 22, pp. 9433-9435, Jun. 2009. [Online]. Available: http://pubs.acs.org/doi/abs/10.1021/jp9010444

[30] J. Geng and H. Jung, "Porphyrin Functionalized Graphene Sheets in Aqueous Suspensions: From the Preparation of Graphene Sheets to Highly Conductive Graphene Films," The Journal of Physical Chemistry $C$, vol. 114, no. 18, pp. 8227-8234, May 2010. [Online]. Available: http://pubs.acs.org/doi/abs/10.1021/jp1008779

[31] R. M. Martin, Electronic structure: basic theory and practical methods. Cambridge University Press, 2004.

[32] P. Hohenberg, W. Kohn, and et.al., "Inhomogeneous electron gas," Physical Review, vol. 136, no. 3B, pp. B864-B871, 1964. [Online]. Available: http://link.aps.org/doi/10.1103/PhysRev.136.B864

[33] W. Kohn, L. Sham, and et.al., "Self-consistent equations including exchange and correlation effects," Physical Review, vol. 140, no. 4A, pp. A1133-A1138, 1965. [Online]. Available: http://link.aps.org/doi/10.1103/PhysRev.140.A1133

[34] L. Hedin, "New method for calculating the one-particle Green's function with application to the electron-gas problem," Physical Review, vol. 139, no. 3A, pp. A796-A823, 1965. [Online]. Available: http://link.aps.org/doi/10.1103/PhysRev.139.A796

[35] S. H. Vosko, L. Wilk, and M. Nusair, "Accurate spin-dependent electron liquid correlation energies for local spin density calculations: a critical analysis," Canadian Journal of Physics, vol. 58, no. 8, pp. 1200-1211, 1980.

[36] J. P. Perdew and A. Zunger, "Self-interaction correction to density-functional approximations for many-electron systems," Phys. Rev. B, vol. 23, 1981.

[37] J. P. Perdew, J. A. Chevary, S. H. Vosko, K. A. Jackson, M. R. Pederson, D. J. Singh, and C. Fiolhais, "Atoms, molecules, solids, and surfaces: Applications of the generalized gradient approximation for exchange and correlation," Phys. Rev. B, vol. 46, 1992. 
[38] J. P. Perdew and Y. Wang, "Accurate and simple analytic representation of the electron-gas correlation energy," Phys. Rev. B, vol. 45, p. 1324413249, 1992.

[39] J. P. Perdew, K. Burke, and M. Ernzerhof, "Generalized Gradient Approximation Made Simple," Phys. Rev. Lett., vol. 77, 1996.

[40] A. Becke, "Density-functional exchange-energy approximation with correct asymptotic behavior," Physical Review A, vol. 38, no. 6, p. 3098, 1988. [Online]. Available: http://pra.aps.org/abstract/PRA/v38/i6/p3098_1

[41] C. Lee, W. Yang, and R. G. Parr, "Correlation: Development of the ColleSalvetti correlation-energy formula into a functional of the electron density," Phys. Rev. B, vol. 37, 1988.

[42] A. D. Becke, "Density-functional exchange-energy approximation with correct asymptotic behavior," Physical Review A, vol. 38, no. 6, p. 3098, 1988.

[43] J. A. Majewski, S. Birner, A. Trellakis, M. Sabathil, and P. Vogl, "Advances in the theory of electronic structure of semiconductors," physica status solidi (c), vol. 1, no. 8, pp. 2003-2027, Jul. 2004. [Online]. Available: http://doi.wiley.com/10.1002/pssc.200404761

[44] A. Becke, "Density-functional thermochemistry. III. The role of exact exchange," J. Chem. Phys., no. 98, 1993.

[45] P. J. Stephens, F. J. Devlin, C. F. Chabalowski, and M. J. Frisch, "Ab Initio Calculation of Vibrational Absorption and Circular Dichroism Spectra Using Density Functional Force Fields," J. Phys. Chem., vol. 98, 1994.

[46] J. Andzelm, E. Wimmer, and D. R. Salahub, Spin Density Functional Approach to the Chemistry of Transition Metal Clusters. [Online]. Available: http://pubs.acs.org/doi/abs/10.1021/bk-1989-0394.ch016

[47] L. Versluis and T. Ziegler, "The determination of molecular structures by density functional theory. The evaluation of analytical energy gradients by numerical integration," The Journal of chemical physics, vol. 88, no. May 2011, p. 322, 1988. [Online]. Available: http://link.aip.org/link/?JCPSA6/88/322/1

[48] N. Ashcroft, Solid State Physics. New York: Holt, Rinehart and Winston, 1976.

[49] M. C. Payne, M. P. Teter, D. C. Allan, T. A. Arias, and J. D. Joannopoulos, "Iterative minimization techniques for ab initio total-energy calculations: molecular dynamics and conjugate gradients," Rev. Mod. Phys., vol. 64, no. 4, pp. 1045-1097, Oct 1992. 
[50] B. Delley, "An all-electron numerical method for solving the local density functional for polyatomic molecules," The Journal of Chemical Physics, vol. 92, no. 1, pp. 508-517, 1990. [Online]. Available: http://link.aip.org/link/?JCP/92/508/1

[51] H. Monkhorst and J. Pack, "Special points for Brillouin-zone integrations," Physical Review B, vol. 13, no. 12, pp. 5188-5192, 1976.

[52] A. Ferretti, B. Bonferroni, A. Calzolari, and M. Buongiorno Nardelli, "An integrated approach to Ab initio electronic transport from maximally-localized Wannier functions." p. 035108, 2004. [Online]. Available: http://www.wanniertransport.org

[53] A. A. Mostofi, J. R. Yates, Y. S. Lee, I. Souza, D. Vanderbilt, and N. Marzari, "Wannier90: A Tool for Obtaining Maximally-Localised Wannier Functions," Computer Physics Communications, vol. 178, no. 9, pp. 685-699, Aug. 2007. [Online]. Available: http://arxiv.org/abs/0708.0650 http://linkinghub.elsevier.com/retrieve/pii/S0010465507004936

[54] C. Delerue and M. Lannoo, Nanostructures: theory and modeling, ser. Nanoscience and technology. Springer, 2004. [Online]. Available: http://books.google.com/books?id=ee8s8ZmyGycC

[55] R. Landauer, "Electrical resistance of disordered one-dimensional lattices," Philosophical Magazine, vol. 21, no. 172, pp. 863-867, Apr. 1970. [Online]. Available: http://www.tandfonline.com/doi/abs/10.1080/14786437008238472

[56] M. Büttiker, "Four-terminal phase-coherent conductance," Physical review letters, vol. 57, no. 14, pp. 1761-1764, 1986. [Online]. Available: http://link.aps.org/doi/10.1103/PhysRevLett.57.1761

[57] M. Buongiorno Nardelli, "Electronic transport in extended systems: Application to carbon nanotubes," Physical Review B, vol. 60, no. 11, pp. 7828-7833, Sep. 1999. [Online]. Available: http://link.aps.org/doi/10.1103/PhysRevB.60.7828 http://prb.aps.org/abstract/PRB/v60/i11/p7828_1

[58] W. Aulbur, M. Städele, and A. Görling, "Exact-exchange-based quasiparticle calculations," Physical Review B, vol. 62, no. 11, p. 7121, 2000. [Online]. Available: http://prb.aps.org/abstract/PRB/v62/i11/p7121_1

[59] M. Brandbyge, J. Mozos, P. Ordejon, J. Taylor, and K. Stokbro, "Density-functional method for nonequilibrium electron transport," Physical Review B, vol. 65, no. 16, p. 165401, Mar. 2002. [Online]. Available: http://prb.aps.org/abstract/PRB/v65/i16/e165401 
[60] G. Wannier, "The structure of electronic excitation levels in insulating crystals," Physical Review, vol. 52, no. 3, p. 191, 1937. [Online]. Available: http://prola.aps.org/abstract/PR/v52/i3/p191_1

[61] W. Kohn, "Analytic properties of Bloch waves and Wannier functions," Physical Review, vol. 115, no. 4, p. 809, 1959. [Online]. Available: http://prola.aps.org/abstract/PR/v115/i4/p809_1

[62] N. Marzari and D. Vanderbilt, "Maximally localized generalized Wannier functions for composite energy bands," Physical Review B, vol. 56, no. 20, pp. 12847-12865, Nov. 1997. [Online]. Available: http://link.aps.org/doi/10.1103/PhysRevB.56.12847

[63] S. F. Boys, "Construction of Some Molecular Orbitals to Be Approximately Invariant for Changes from One Molecule to Another," Reviews of modern physics, vol. 32, no. 3, pp. 296-299, 1960.

[64] N. Marzari and I. Souza, "An introduction to maximally-localized Wannier functions," Psi-K Newsletter, pp. 129-168, 2003. [Online]. Available: http://www.wannier.org/papers/MSVpsik.pdf

[65] A. Calzolari, N. Marzari, I. Souza, and M. Buongiorno Nardelli, "Ab initio transport properties of nanostructures from maximally localized Wannier functions," Physical Review B, vol. 69, no. 3, p. 035108, Jan. 2004. [Online]. Available: http://link.aps.org/doi/10.1103/PhysRevB.69.035108

[66] P. Giannozzi, S. Baroni, N. Bonini, M. Calandra, R. Car, C. Cavazzoni, D. Ceresoli, G. L. Chiarotti, M. Cococcioni, I. Dabo, A. Dal Corso, S. de Gironcoli, S. Fabris, G. Fratesi, R. Gebauer, U. Gerstmann, C. Gougoussis, A. Kokalj, M. Lazzeri, L. Martin-Samos, N. Marzari, F. Mauri, R. Mazzarello, S. Paolini, A. Pasquarello, L. Paulatto, C. Sbraccia, S. Scandolo, G. Sclauzero, A. P. Seitsonen, A. Smogunov, P. Umari, and R. M. Wentzcovitch, "QUANTUM ESPRESSO: a modular and open-source software project for quantum simulations of materials," Journal of Physics: Condensed Matter, vol. 21, no. 39, p. 395502, Sep. 2009. [Online]. Available: http://stacks.iop.org/0953$8984 / 21 / \mathrm{i}=39 / \mathrm{a}=395502$ ?key=crossref.c21336c286fa6d3db893262ae3f6e151

[67] D. Williams and C. Carter, Transmission electron microscopy: Basics. Plenum Press, 2009, no. let. 1. [Online]. Available: http://books.google.com/books?id=Cbwf0OkzDvMC

[68] J. Spence and J. M. Zou, Electron Microdiffraction. New York: Plenum Press, 1992.

[69] E. J. Kirkland, Advanced Computing in Electron Microscopy. Springer, 1998. 
[70] J. M. Cowley and A. F. Moodie, "The scattering of electrons by atoms and crystals. i. a new theoretical approach," Acta Crystallographica, vol. 10, no. 10, pp. 609-619, Oct 1957. [Online]. Available: http://dx.doi.org/10.1107/S0365110X57002194

[71] K. Ishizuka and N. Uyeda, "A new theoretical and practical approach to the multislice method," Acta Crystallographica Section $A$, vol. 33, no. 5, pp. 740-749, Sep 1977. [Online]. Available: http://dx.doi.org/10.1107/S0567739477001879

[72] O. Scherzer, "Sphärische und chromatische korrektur von elektronenlinsen," Optik, vol. 2, pp. 114-132, 1948.

[73] E. Prince, Mathematical, physical and chemical tables, ser. International tables for crystallography. Published for the International Union of Crystallography by Springer, 2006. [Online]. Available: http://books.google.com/books?id=60FoFEGyShIC

[74] H. Nyquist, "Certain topics in telegraph transmission theory," Proceedings of the IEEE, vol. 90, no. 2, pp. 280-305, 2002. [Online]. Available: http://ieeexplore.ieee.org/lpdocs/epic03/wrapper.htm?arnumber $=989875$

[75] MATLAB, version 7.10.0 (R2010a). Natick, MA: The MathWorks Inc., 2010.

[76] S. Hovmoeller, "Crisp: crystallographic image processing on a personal computer," Ultramicroscopy, vol. 41, no. 1-3, pp. 121 - 135, 1992. [Online]. Available: http://www.sciencedirect.com/science/article/pii/030439919290102P

[77] J. C. Meyer, C. Kisielowski, R. Erni, M. D. Rossell, M. F. Crommie, and A. Zettl, "Direct imaging of lattice atoms and topological defects in graphene membranes." Nano letters, vol. 8, no. 11, pp. 3582-6, Nov. 2008. [Online]. Available: http://www.ncbi.nlm.nih.gov/pubmed/18563938

[78] C. Girit, J. C. Meyer, R. Erni, M. D. Rossell, C. Kisielowski, L. Yang, C.-H. Park, M. F. Crommie, M. L. Cohen, S. G. Louie, and A. Zettl, "Graphene at the Edge: Stability and Dynamics," Science, vol. 323, no. 5922, pp. 1705-1708, 2009 .

[79] M. Han, B. Özyilmaz, Y. Zhang, and P. Kim, "Energy BandGap Engineering of Graphene Nanoribbons," Physical Review Letters, vol. 98, no. 20, p. 206805, May 2007. [Online]. Available: http://link.aps.org/doi/10.1103/PhysRevLett.98.206805

[80] R. Erni, M. D. Rossell, M.-T. Nguyen, S. Blankenburg, D. Passerone, P. Hartel, N. Alem, K. Erickson, W. Gannett, and A. Zettl, "Stability and dynamics of 
small molecules trapped on graphene," Physical Review B, vol. 82, no. 16, p. 165443.

[81] O. Leenaerts, B. Partoens, and F. Peeters, "Adsorption of small molecules on graphene," Microelectronics Journal, vol. 40, no. 4-5, pp. 860-862, Apr. 2009. [Online]. Available: http://linkinghub.elsevier.com/retrieve/pii/S0026269208005582

[82] C. Neto, V. N. Kotov, J. Nilsson, V. M. Pereira, N. M. R. Peres, B. Uchoa, and A. Neto, "Adatoms in graphene," Solid State Communication, vol. 149, no. 27-28, pp. 1094-1100, Jul. 2009. [Online]. Available: http://arxiv.org/abs/0812.2072

[83] R. Zacharia, H. Ulbricht, and T. Hertel, "Interlayer cohesive energy of graphite from thermal desorption of polyaromatic hydrocarbons," Physical Review B, vol. 69, no. 15, p. 155406, Apr. 2004. [Online]. Available: http://prb.aps.org/abstract/PRB/v69/i15/e155406

[84] N. Ooi, A. Rairkar, and J. Adams, "Density functional study of graphite bulk and surface properties," Carbon, vol. 44, no. 2, pp. 231-242, Feb. 2006. [Online]. Available: http://www.sciencedirect.com/science/article/pii/S0008622305004719 http://linkinghub.elsevier.com/retrieve/pii/S0008622305004719

[85] C. N. R. Rao, A. K. Sood, K. S. Subrahmanyam, and A. Govindaraj, "Graphene: the new two-dimensional nanomaterial." Angewandte Chemie (International ed. in English), vol. 48, no. 42, pp. 7752-77, Jan. 2009. [Online]. Available: http://onlinelibrary.wiley.com/doi/10.1002/anie.200901678/full

[86] A. Grüneis, C. Attaccalite, L. Wirtz, H. Shiozawa, R. Saito, T. Pichler, and A. Rubio, "Tight-binding description of the quasiparticle dispersion of graphite and few-layer graphene," Physical Review B, vol. 78, no. 20, p. 205425, Nov. 2008. [Online]. Available: http://prb.aps.org/abstract/PRB/v78/i20/e205425

[87] H. Raza and E. C. Kan, "Field modulation in bilayer graphene band structure," Journal of Physics: Condensed Matter, vol. 21, no. 10, p. 102202, Mar. 2009. [Online]. Available: http://stacks.iop.org/0953$8984 / 21 / \mathrm{i}=10 / \mathrm{a}=102202$ ?key $=$ crossref.345b4c28ffec1e23aafa486d0532e286

[88] E. McCann, "Asymmetry gap in the electronic band structure of bilayer graphene," Physical Review B, vol. 74, no. 16, p. 161403, Oct. 2006. [Online]. Available: http://prb.aps.org/abstract/PRB/v74/i16/e161403

[89] G. Giovannetti, P. Khomyakov, G. Brocks, P. Kelly, and J. van den Brink, "Substrate-induced band gap in graphene on hexagonal boron 
nitride: $\mathrm{Ab}$ initio density functional calculations," Physical Review B, vol. 76, no. 7, p. 073103, Aug. 2007. [Online]. Available: http://prb.aps.org/abstract/PRB/v76/i7/e073103

[90] R. Chen, P. Trucano, and R. Stewart, "The valence-charge density of graphite," Acta Crystallographica Section A: Crystal Physics, Diffraction, Theoretical and General Crystallography, vol. 33, no. 5, pp. 823-828, 1977. [Online]. Available: http://scripts.iucr.org/cgi-bin/paper?a14232

[91] A. G. Garcia, S. E. Baltazar, A. H. R. Castro, J. F. P. Robles, and A. Rubio, "Influence of $\mathrm{S}$ and $\mathrm{P}$ Doping in a Graphene Sheet," Journal of Computational and Theoretical Nanoscience, vol. 5, no. 11, pp. 2221-2229, Nov. 2008. [Online]. Available: $\quad$ http://openurl.ingenta.com/content/xref?genre=article\&issn=1546$1955 \&$ volume $=5 \&$ issue $=11 \&$ spage $=2221$

[92] K. Imani, M. R. Abolhassani, and Sabouri-Dodaran A. A., "Electronic transport calculation of adsorbate $\mathrm{NO} 2$ and $\mathrm{NO}$ molecules on graphene using Maximally Localized Wannier functions," The European Physical Journal B, vol. 74, no. 1, pp. 135-138, Mar. 2010. [Online]. Available: http://www.springerlink.com/index/10.1140/epjb/e2010-00075-8

[93] L. Ci, Z. Xu, L. Wang, W. Gao, F. Ding, K. Kelly, B. Yakobson, and P. Ajayan, "Controlled nanocutting of graphene," Nano Research, vol. 1, no. 2, pp. 116-122, 2008. [Online]. Available: http://www.springerlink.com/index/3572925JQ1313666.pdf

[94] V. Barone, O. Hod, and G. E. Scuseria, "Electronic Structure and Stability of Semiconducting Graphene Nanoribbons," Nano Letters, vol. 6, no. 12, pp. $2748-2754,2006$.

[95] Z. F. Wang, Q. W. Shi, Q. Li, X. Wang, J. G. Hou, H. Zheng, Y. Yao, and J. Chen, "Z-shaped graphene nanoribbon quantum dot device," Applied Physics Letters, vol. 91, no. 5, p. 053109, 2007. [Online]. Available: http://link.aip.org/link/APPLAB/v91/i5/p053109/s1\&Agg=doi

[96] V. Tozzini and V. Pellegrini, "Electronic structure and Peierls instability in graphene nanoribbons sculpted in graphane," Physical Review $B$, vol. 81, no. 11, pp. 22-25, Mar. 2010. [Online]. Available: http://link.aps.org/doi/10.1103/PhysRevB.81.113404

[97] Y. Niimi, T. Matsui, H. Kambara, K. Tagami, M. Tsukada, and H. Fukuyama, "Scanning tunneling microscopy and spectroscopy of the electronic local density of states of graphite surfaces near monoatomic step edges," Physical 
Review B, vol. 73, no. 8, pp. 085421 - 085 428, Feb. 2006. [Online]. Available: http://link.aps.org/doi/10.1103/PhysRevB.73.085421

[98] N. Mingo, "Calculation of Si nanowire thermal conductivity using complete phonon dispersion relations," Physical Review B, vol. 68, no. 11, p. 113308, Sep. 2003. [Online]. Available: http://prb.aps.org/abstract/PRB/v68/i11/e113308

[99] Z. W. Tan, J.-S. Wang, and C. K. Gan, "First-principles study of heat transport properties of graphene nanoribbons." Nano letters, vol. 11, no. 1, pp. 214-9, Jan. 2011. [Online]. Available: http://www.ncbi.nlm.nih.gov/pubmed/21158401

[100] K. Schwab, E. Henriksen, J. Worlock, and M. Roukes, "Measurement of the quantum of thermal conductance," Nature, vol. 404, no. 6781, pp. 974-7, Apr. 2000. [Online]. Available: http://www.ncbi.nlm.nih.gov/pubmed/10801121

[101] D. Santamore and M. Cross, "Effect of surface roughness on the universal thermal conductance," Physical Review B, vol. 63, no. 18, p. 184306, Apr. 2001. [Online]. Available: http://link.aps.org/doi/10.1103/PhysRevB.63.184306

[102] E. Munoz, J. Lu, and B. I. Yakobson, "Ballistic thermal conductance of graphene ribbons," Nano letters, vol. 10, no. 5, pp. 1652-1656, 2010. [Online]. Available: http://pubs.acs.org/doi/abs/10.1021/nl904206d

[103] Y. Huang, J. Wu, and K. Hwang, "Thickness of graphene and single-wall carbon nanotubes," Physical Review B, vol. 74, no. 24, pp. 1-9, Dec. 2006. [Online]. Available: http://link.aps.org/doi/10.1103/PhysRevB.74.245413

[104] L. Wang, Q. Zheng, J. Liu, and Q. Jiang, "Size Dependence of the Thin-Shell Model for Carbon Nanotubes," Physical Review Letters, vol. 95, no. 10, pp. 2-5, Sep. 2005. [Online]. Available: http://link.aps.org/doi/10.1103/PhysRevLett.95.105501

[105] D. Brenner, O. Shenderova, J. Harrison, S. Stuart, B. Ni, and S. Sinnott, "A second-generation reactive empirical bond order (REBO) potential energy expression for hydrocarbons," Journal of Physics: Condensed Matter, vol. 14, p. 783, 2002. [Online]. Available: http://iopscience.iop.org/0953-8984/14/4/312

[106] Z. Aksamija and I. Knezevic, "Lattice thermal conductivity of graphene nanoribbons: Anisotropy and edge roughness scattering," Applied Physics Letters, vol. 98, no. 14, p. 141919, 2011. [Online]. Available: http://link.aip.org/link/APPLAB/v98/i14/p141919/s1\&Agg=doi

[107] S. Ghosh, I. Calizo, D. Teweldebrhan, E. P. Pokatilov, D. L. Nika, A. A. Balandin, W. Bao, F. Miao, and C. N. Lau, "Extremely high thermal conductivity of graphene: Prospects for thermal management applications in nanoelectronic 
circuits," Applied Physics Letters, vol. 92, no. 15, p. 151911, 2008. [Online]. Available: http://link.aip.org/link/APPLAB/v92/i15/p151911/s1\&Agg=doi

[108] W. J. Evans, L. Hu, and P. Keblinski, "Thermal conductivity of graphene ribbons from equilibrium molecular dynamics: Effect of ribbon width, edge roughness, and hydrogen termination," Applied Physics Letters, vol. 96, no. 20, p. 203112, 2010. [Online]. Available: http://link.aip.org/link/APPLAB/v96/i20/p203112/s1\&Agg=doi

[109] Z. Aksamija and I. Knezevic, "Lattice thermal conductivity of graphene nanoribbons: Anisotropy and edge roughness scattering," Applied Physics Letters, vol. 98, no. 14, pp. 141913-141919, 2011.

[110] S. J. Clark, M. D. Segall, C. J. Pickard, P. J. Hasnip, M. I. J. Probert, K. Refson, and M. C. Payne, "First principles methods using CASTEP," Zeitschrift für Kristallographie, vol. 220, no. 5-6-2005, pp. 567-570, May 2005. [Online]. Available: http://www.oldenbourglink.com/doi/abs/10.1524/zkri.220.5.567.65075

[111] J. D. Gale and A. L. Rohl, "The General Utility Lattice Program (GULP)," Molecular Simulation, vol. 29, no. 5, pp. 291-341, 2003.

[112] J. Maultzsch, S. Reich, C. Thomsen, H. Requardt, Ordej, Oacute, and P. N, "Phonon Dispersion in Graphite," Physical Review Letters, vol. 92, no. 7, p. 75501, 2004.

[113] C. Gan and D. Srolovitz, "First-principles study of graphene edge properties and flake shapes," Physical Review B, vol. 81, no. 12, p. 125445, Mar. 2010. [Online]. Available: http://prb.aps.org/abstract/PRB/v81/i12/e125445

[114] R. Saito, G. Dresselhaus, M. Dresselhaus, and K. (Firm), Physical properties of carbon nanotubes. London: Imperial College Press London, 1998, vol. 3. [Online]. Available: http://books.google.com/books?id=w5oHCWhA2EQC

[115] H. Zabel, "Phonons in layered compounds," Journal of Physics: Condensed Matter, vol. 13, no. 34, pp. 76797690, Aug. 2001. [Online]. Available: http://stacks.iop.org/0953$8984 / 13 / \mathrm{i}=34 / \mathrm{a}=313$ ?key=crossref.26db543b0d65590a110bf90db60af84e

[116] J. Ziman, Electrons and Phonons: The Theory of Transport Phenomena in Solids. New York: Oxford University Press, 1960.

[117] T. Yamamoto, S. Konabe, J. Shiomi, and S. Maruyama, "Crossover from Ballistic to Diffusive Thermal Transport in Carbon Nanotubes," Applied Physics Express, vol. 2, no. 9, p. 095003, Aug. 2009. [Online]. Available: http://online.ipap.jp/link?APEX/2/095003/ 
[118] G. Srivastava, The physics of phonons. A. Hilger, 1990. [Online]. Available: http://books.google.com/books?id=OE-bHd2gzVgC

[119] Z. Wang, R. Xie, C. T. Bui, D. Liu, X. Ni, B. Li, and J. T. L. Thong, "Thermal Transport in Suspended and Supported Few-Layer Graphene." Nano letters, pp. 113-118, Dec. 2010. [Online]. Available: http://www.ncbi.nlm.nih.gov/pubmed/21142193

[120] D. C. Elias, R. R. Nair, T. M. G. Mohiuddin, S. V. Morozov, P. Blake, M. P. Halsall, A. C. Ferrari, D. W. Boukhvalov, M. I. Katsnelson, A. Geim, and K. Novoselov, "Control of graphene's properties by reversible hydrogenation: evidence for graphane." Science (New York, N.Y.), vol. 323, no. 5914, pp. 610-3, Jan. 2009. [Online]. Available: http://www.sciencemag.org/content/323/5914/610

[121] M. Z. S. Flores, P. A. S. Autreto, S. B. Legoas, and D. S. Galvao, "Graphene to graphane: a theoretical study." Nanotechnology, vol. 20, no. 46, p. 465704, Nov. 2009. [Online]. Available: http://www.ncbi.nlm.nih.gov/pubmed/19843995

[122] M. Quintana, K. Spyrou, M. Grzelczak, W. R. Browne, P. Rudolf, and M. Prato, "Functionalization of graphene via 1,3-dipolar cycloaddition." ACS nano, vol. 4, no. 6, pp. 3527-33, Jun. 2010. [Online]. Available: http://www.ncbi.nlm.nih.gov/pubmed/20503982

[123] L.-H. Liu and M. Yan, "Simple method for the covalent immobilization of graphene." Nano letters, vol. 9, no. 9, pp. 3375-8, Sep. 2009. [Online]. Available: http://www.pubmedcentral.nih.gov/articlerender.fcgi?artid=2740804

[124] L.-H. Liu, G. Zorn, D. G. Castner, R. Solanki, M. M. Lerner, and M. Yan, "A simple and scalable route to wafer-size patterned graphene," Journal of Materials Chemistry, vol. 20, no. 24, p. 5041, 2010. [Online]. Available: http://xlink.rsc.org/?DOI=c0jm00509f

[125] L.-H. Liu, G. Nandamuri, R. Solanki, and M. Yan, "Electrical properties of covalently immobilized single-layer graphene devices." Journal of nanoscience and nanotechnology, vol. 11, no. 2, pp. 1288-92, Feb. 2011. [Online]. Available: http://www.ncbi.nlm.nih.gov/pubmed/21456173

[126] O. Leenaerts, B. Partoens, and F. Peeters, "Adsorption of $\mathrm{H} 2 \mathrm{O}$, NH3, $\mathrm{CO}$, NO2, and $\mathrm{NO}$ on graphene: A first-principles study," Physical Review B, vol. 77, no. 12, p. 125416, Mar. 2008. [Online]. Available: http://prb.aps.org/abstract/PRB/v77/i12/e125416 http://link.aps.org/doi/10.1103/PhysRevB.77.125416 
[127] — "Paramagnetic adsorbates on graphene: A charge transfer analysis," Applied Physics Letters, vol. 92, no. 24, p. 243125, 2008. [Online]. Available: http://link.aip.org/link/?APPLAB/92/243125/1

[128] R. Erni, M. Rossell, M.-T. Nguyen, S. Blankenburg, D. Passerone, P. Hartel, N. Alem, K. Erickson, W. Gannett, and A. Zettl, "Stability and dynamics of small molecules trapped on graphene," Physical Review B, vol. 82, no. 16, p. 165443, Oct. 2010. [Online]. Available: http://prb.aps.org/abstract/PRB/v82/i16/e165443 http://link.aps.org/doi/10.1103/PhysRevB.82.165443

[129] V. Zólyomi, A. Rusznyák, J. Koltai, J. Kürti, and C. J. Lambert, "Functionalization of graphene with transition metals," Physica Status Solidi (B), vol. 247, no. 11-12, pp. 2920-2923, Dec. 2010. [Online]. Available: http://doi.wiley.com/10.1002/pssb.201000168

[130] H. Park, J. Zhao, and J. P. Lu, "Effects of sidewall functionalization on conducting properties of single wall carbon nanotubes." Nano letters, vol. 6, no. 5, pp. 916-9, May 2006. [Online]. Available: http://www.ncbi.nlm.nih.gov/pubmed/16683825

[131] S.-M. Dubois, Z. Zanolli, X. Declerck, and J.-C. Charlier, "Electronic properties and quantum transport in Graphene-based nanostructures," The European Physical Journal B-Condensed Matter and Complex Systems, vol. 72, no. 1, pp. 1-24, Oct. 2009. [Online]. Available: http://www.springerlink.com/index/U134V31550744050.pdf

[132] A. P. Saxena, M. Deepa, A. G. Joshi, S. Bhandari, and A. K. Srivastava, "Poly(3,4-ethylenedioxythiophene)-ionic liquid functionalized graphene/reduced graphene oxide nanostructures: improved conduction and electrochromism." ACS applied materials \& interfaces, vol. 3, no. 4, pp. 1115-26, Apr. 2011. [Online]. Available: http://pubs.acs.org/doi/pdf/10.1021/am101255a

[133] A. Kutana and K. P. Giapis, "Analytical carbon-oxygen reactive potential." The Journal of chemical physics, vol. 128, no. 23, p. 234706, Jun. 2008. [Online]. Available: http://www.ncbi.nlm.nih.gov/pubmed/18570518

[134] K. P. Loh, Q. Bao, P. K. Ang, and J. Yang, "The chemistry of graphene," Journal of Materials Chemistry, vol. 20, no. 12, p. 2277, 2010. [Online]. Available: http://xlink.rsc.org/?DOI=b920539j

[135] K. Suggs, D. Reuven, and X. Wang, "Electronic Properties of Cycloaddition-Functionalized Graphene," The Journal of Physical Chemistry $C$, vol. 115, no. 8, p. 33133317, 2011. [Online]. Available: http://pubs.acs.org/doi/abs/10.1021/jp111637b 
[136] P. Fuentealba and R. Contreras, "Fukui function in chemistry," ChemInform, vol. 34, no. 29, pp. no-no, 2003. [Online]. Available: http://onlinelibrary.wiley.com/doi/10.1002/chin.200329281/full

[137] D. V. Kosynkin, A. L. Higginbotham, A. Sinitskii, J. R. Lomeda, A. Dimiev, B. K. Price, and J. M. Tour, "Longitudinal unzipping of carbon nanotubes to form graphene nanoribbons." Nature, vol. 458, no. 7240, pp. 872-6, Apr. 2009. [Online]. Available: http://www.ncbi.nlm.nih.gov/pubmed/19370030

[138] A. D. McNaught and A. Wilkinson, IUPAC. Compendium of Chemical Terminology, 2nd ed. (the "Gold Book"). Oxford: Blackwell Scientific Publications, 2006.

[139] R. Toreki, "Organometallic hypertextbook." [Online]. Available: http://www.ilpi.com/organomet/carbonyl.html

[140] D. Astruc, Organometallic chemistry and catalysis. Springer Verlag, May 2007, vol. 30 , no. 3 .

[141] R. L. Brandon, J. H. Osiecki, and A. Ottenberg, "The Reactions of Metallocenes with Electron Acceptors1a," The Journal of Organic Chemistry, vol. 31, no. 4, pp. 1214-1217, 1966.

[142] C. N. R. Rao, K. S. Subrahmanyam, H. S. S. Ramakrishna Matte, B. Abdulhakeem, A. Govindaraj, B. Das, P. Kumar, A. Ghosh, and D. J. Late, "A study of the synthetic methods and properties of graphenes," Science and Technology of Advanced Materials, vol. 11, no. 5, p. 054502, Oct. 2010. [Online]. Available: http://stacks.iop.org/1468$6996 / 11 / \mathrm{i}=5 / \mathrm{a}=054502 ? \mathrm{key}=$ crossref.98836d16349f47f40eebcdfeb42c16c7

[143] C. Elschenbroich and R. Möckel, "Bis( $\eta 6$-naphthalin)chrom(0)," Angewandte Chemie, vol. 89, no. 12, pp. 908-909, Dec. 1977. [Online]. Available: http://doi.wiley.com/10.1002/ange.19770891225

[144] E. P. Kundig and P. L. Timms, "Metal atom preparation and ligand displacement reactions of bisnaphthalenechromium and related compounds," Journal of the Chemical Society, Chemical Communications, no. 24, pp. 912-913, 1977.

[145] V. I. Minkin, B. Y. Simkin, and R. M. Minyaev, Teoriya stroieniya molekul (in Russian). Rostov-on-Don: Phoenix, 1997.

[146] K. F. Mak, C. H. Lui, J. Shan, and T. F. Heinz, "Observation of an ElectricField-Induced Band Gap in Bilayer Graphene by Infrared Spectroscopy," Physical Review Letters, vol. 102, no. 25, p. 256405, 2009. 
[147] A. V. Krasheninnikov, P. O. Lehtinen, A. S. Foster, P. Pyykko, and R. M. Nieminen, "Embedding Transition-Metal Atoms in Graphene: Structure, Bonding, and Magnetism," Physical Review Letters, vol. 102, no. 12, p. 126807, 2009.

[148] M. Wu, C. Cao, and J. Z. Jiang, "Electronic structure of substitutionally Mn-doped graphene," New Journal of Physics, vol. 12, no. 6, p. 063020, Jun. 2010. [Online]. Available: http://stacks.iop.org/13672630/12/i=6/a=063020?key=crossref.e383a89116f33311b3466db96cbeeebb

[149] S. Blügel and P. H. Dederichs, "Ferromagnetism and Antiferromagnetism of $3 \mathrm{~d}$ Metal Overlayers on Noble-Metal Substrates," Europhysics Letters (EPL), vol. 9, no. 6, pp. 597602, Jul. 1989. [Online]. Available: http://stacks.iop.org/0295$5075 / 9 / \mathrm{i}=6 / \mathrm{a}=018 ? \mathrm{key}=$ crossref.dd6f0872dfd7f41aea95fbeb21e97390

[150] T. Yuen, C. L. Lin, L. Pan, X. Huang, and J. Li, "Magnetic properties of a metal-organic antiferromagnet $\mathrm{Mn}(\mathrm{hfipbb}) \mathrm{py}(\mathrm{H} 2 \mathrm{O}) 0.5$," Journal of Applied Physics, vol. 99, no. 8, p. 08J501, 2006. [Online]. Available: http://link.aip.org/link/JAPIAU/v99/i8/p08J501/s1\&Agg=doi

[151] T. A. Albright and B. K. Carpenter, "Conformational effects of nucleophilic and electrophilic attack on (arene)chromium tricarbonyl complexes," Inorganic Chemistry, vol. 19, no. 10, pp. 3092-3097, Oct. 1980. [Online]. Available: http://pubs.acs.org/doi/abs/10.1021/ic50212a053

[152] P. Plachinda, S. Rouvimov, and R. Solanki, "Structure analysis of CVD graphene films based on HRTEM contrast simulations," physica status solidi (a), vol. 208, no. 11, pp. 2681-2687, Jul. 2011. [Online]. Available: http://doi.wiley.com/10.1002/pssa.201127067 http://onlinelibrary.wiley.com/doi/10.1002/pssa.201127067/full

[153] P. Plachinda, D. R. Evans, and R. Solanki, "Electronic properties of metal-arene functionalized graphene," The Journal of Chemical Physics, vol. 135, no. 4, p. 044103, 2011. [Online]. Available: http://link.aip.org/link/JCPSA6/v135/i4/p044103/s1\&Agg=doi

[154] B. Delley, "From molecules to solids with the DMol approach," The Journal of Chemical Physics, vol. 113, no. 18, p. 7756, 2000. [Online]. Available: http://link.aip.org/link/?JCPSA6/113/7756/1

[155] C. Lee, W. Yang, and R. G. Parr, "Development of the Colle-Salvetti correlation-energy formula into a functional of the electron density," Physical Review B, vol. 37, no. 2, p. 785, 1988. 


\section{APPENDIX}

\section{Standard parameters for the DFT calculations}

\section{A.1 Standard parameters for the DFT calculations}

\section{A.1.1 Geometry optimization}

The calculations were conducted within the framework of the DFT theory as implemented in the $\mathrm{DMol}^{3}$ package[154]. The generalized gradient approximation (GGA) in BLYP $[40,155]$ exchange-correlation parameterization was used for both final geometry optimization and band structure calculation. Initial geometry optimization was performed using the local density approximation (LDA) approximation with the Vosko-Wilk-Nusair (VWN) [35] correlation functional.

A 6x6 graphene supercell with a vacuum space of $11.5 \AA$ normal to graphene plane was used. Geometry optimization convergence criterion was satisfied when the total energy change was less than of $3 \times 10^{-5} \mathrm{Ha}$. Only one k-point $(\Gamma)$ was used throughout the structural calculations since the distance between neighboring k-points was only $0.0771 / \AA$ due to a large supercell choice.

\section{A.1.2 Band structure}

For the band structure computation the k-path selected was $\Gamma-\mathrm{M}-\mathrm{K}-\Gamma$ with 24 , 20, and 40 k-points on each segment correspondingly.

Although the GGA approach systematically underestimates the band gaps, we are primarily interested in the mechanism of gap opening. For that purpose the GGA approach is expected to provide qualitatively correct information. A more precise GW 
approach is very costly on this system, consisting of a total of 94 atoms. The DMol ${ }^{3}$ package utilizes numerical orbital basis set for the radial part of the wave function, centred on the atoms allowing including a thick vacuum layer without increase of the computation time. 